\title{
Investigating Batoid-Inspired Propulsion: The Development, Testing, and Performance Analysis of a Tensegrity-Based Robotic Fin for Underwater Locomotion
}

A Dissertation Presented to the

Faculty of the School of Engineering and Applied Science

University of Virginia

\author{
In Partial Fulfillment \\ of the Requirements for the Degree of \\ Doctor of Philosophy \\ Mechanical and Aerospace Engineering
}

by

Trevor Hayes Kemp

May 2014 


\begin{abstract}
APPROVAL SHEET
The dissertation is submitted in partial fulfillment of the requirements for the degree of

Doctor of Philosophy in Mechanical and Aerospace Engineering
\end{abstract}

Trevor H. Kemp

This dissertation has been read and approved by the Examining Committee:

Hilary Bart-Smith, Advisor

Hossein Haj-Hariri, Chairman

Gavin T. Garner

Silvia S. Blemker

Haibo Dong

Accepted for the School of Engineering and Applied Science:

James H. Aylor, Dean

School of Engineering and Applied Science

May 2014 


\begin{abstract}
Unmanned underwater vehicles have become an increasingly important tool for research, industry, and the military to perform surveying, monitoring, exploratory, and other functions. They are conventionally designed with rigid hulls and rotary propellers that utilize steady hydrodynamic principles. This is in contrast to biological swimmers, which generally use flexible bodies and appendages to take advantage of unsteady hydrodynamics. As a result, fish and other swimming animals are notably much more maneuverable and efficient compared to conventionally-designed vehicles. This stark difference motivates the development of biologically-inspired designs that can meet or exceed performance seen in nature. Recently, manta rays (and batoid rays in general) have been identified as an ideal platform for an efficient, high endurance, maneuverable and stealthy underwater vehicle. Towards the goal of creating such a vehicle, this study is specifically aimed at reproducing the major kinematic features of oscillatory batoid rays by developing a tensegrity-based robotic pectoral fin and quantifying the swimming performance of this fin.

In this work, the structural mechanics of cable-clustered active tensegrity beams are experimentally validated, so that analytical predictions for their response to external loads can be used in the design of a tensegrity-based robotic pectoral fin. In order to quantify the relationship between kinematic parameters and performance in ray-like swimming, a tensegrity-based robotic fin, capable of actively producing large span-wise bending and passively producing chord-wise curvature, is developed. Two types of experimental hydrodynamic tests are performed in a water tunnel: constrained tests that measure net thrust and propulsive efficiency; and unconstrained tests that measure velocity and free-swimming economy. Constrained tests demonstrate that the simple fin design can produce significant net thrust that is strongly correlated to flapping frequency. The maximum efficiency of this heaving motion is
\end{abstract}


relatively low, so when compared to a chord-wise rigid fin, this suggests that solely adding chord-wise compliance is not beneficial for maximum propulsive efficiency, although passive flexibility seems to be important for broadening the operating range of highest efficiency. Unconstrained tests demonstrate that free-swimming velocity is correlated to both flapping frequency and amplitude. Importantly though, high swimming velocities come at the cost of low economy, indicating an inherent operational trade-off between transport time and energy usage. Kinematic parameters matching biological observations produce free-swimming velocities that are similar to batoid rays, but increased kinematic complexity is expected to improve both efficiency and economy. Flexibility is shown to be an important design parameter for flapping propulsors, with a compliant artificial skin showing enhanced swimming speeds and economies compared to a stiff skin. The experiments show that while Strouhal number (a nondimensional frequency) is correlated with an operating range of maximum efficiency, it does not uniquely correlate to peak values of economy, indicating that caution should be exercised when statements are made about the role of Strouhal number in free-swimming performance. Overall, this study demonstrates that active tensegrity structures can be effectively used to reproduce biologically-relevant kinematics and shows promise for biologically-inspired flapping fins in the application of underwater vehicles. 


\section{Acknowledgements}

If I have seen further, it is by standing on the shoulders of giants.

- Sir Isaac Newton

In this work, I have attempted to contribute some incremental level of understanding about batoid ray swimming. I owe any measure of success in this endeavor to my predecessors and colleagues in the field, without whom I would be lost. For

example, the lucid and at times lighthearted writings of Steven Vogel taught me much about the fundamentals of bio-fluid mechanics, and I was particularly inspired by Carl Heine, who also tried to learn from the rays, documenting his journey in a dissertation. I want to thank all my fellow graduate students of the BIER lab at UVA for their companionship, commiseration, and positive support, especially Russell Biagi for his critical thoughts and attention to detail, and Tom Bliss for all the "gerdunkins" (Gedanken). I would like to thank Keith Moored not only for laying the groundwork of my entire research project, but also for being a great friend and mentor. As I developed prototypes, Lewis Steva was always very helpful in the machine shop, not so much for doing something for me, but for showing me how to do it myself. I owe a great deal to my advisor, Hilary Bart-Smith, for her support intellectually, critically, financially and otherwise. When she first told me she was starting up a project to make robotic manta rays, no further convincing was needed. I'm very appreciative for the experience of this unique opportunity and I was honored to work on the MURI project with her, Hossein Haj-Hariri, Frank Fish, Lex Smits and other collaborators. Finally, I would like to thank all my other friends (in the department and elsewhere) and my family for their support while I made the journey through graduate school. 


\section{Contents}

List of Figures $\quad$ vi

Nomenclature viii

\begin{tabular}{lll}
\hline & Introduction & 1
\end{tabular}

2 Background $\quad 6$

2.1 Underwater Vehicle Technology . . . . . . . . . . . . . . . . . . . 6

2.2 Taking Inspiration from Biology . . . . . . . . . . . . . . . . . . . . . 9

2.2 .1 Biological Observations . . . . . . . . . . . . . . . . . . . . . . . . 9

2.2 .2 Biologically-Inspired Propulsors . . . . . . . . . . . . . . . . . 16

2.3 Active Tensegrity . . . . . . . . . . . . . . . . . 25

3 Validating Tensegrity Mechanics 29

3.1 Tensegrity Mechanics . . . . . . . . . . . . . . . . . . . . . . . . 30

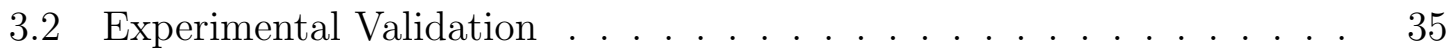

3.2 .1 Test Setup . . . . . . . . . . . . . . . . . . . . . . . . . . . . . . . . . . . . . . 45

3.2 .2 Experimental Procedure . . . . . . . . . . . . . . . . . . 41

3.2 .3 Results and Discussion . . . . . . . . . . . . . . . . . . . . . . 42

4 Robotic Fin Design

4.1 Modified Antagonistic Actuation . . . . . . . . . . . . . . . . 53

4.2 Fin Concept . . . . . . . . . . . . . . . . . . . . . . . . . . . . . . . . 71

4.3 Fluid Loading Model . . . . . . . . . . . . . . . . . . . . . . . . . . . 78

4.4 Implementation of an Active Tensegrity Beam . . . . . . . . . . . . . 83

4.5 Actuation Results . . . . . . . . . . . . . . . . . . . . . . . . . 94

\begin{tabular}{|lll}
5 & Hydrodynamic Experiments & 101
\end{tabular}

5.1 Experimental Setup . . . . . . . . . . . . . . . . . . . . . 102

5.1 .1 Equipment. . . . . . . . . . . . . . . . . . . . . . . 102

5.1 .2 Experimental Procedures . . . . . . . . . . . . . . . . . . . 106

5.1 .3 Performance Calculations . . . . . . . . . . . . . . . . . . . . 110

5.2 Results and Discussion . . . . . . . . . . . . . . . . . . . . . . . . . 114

5.2 .1 Amplitude Measurements . . . . . . . . . . . . . . . . . . . . 114

5.2 .2 Constrained Tests: Surface Wave Suppression . . . . . . . . . 119

5.2 .3 Constrained Tests: Flow Speed Variation . . . . . . . . . . . . 126 
5.2 .4 Unconstrained Tests: Speed and Economy . . . . . . . . . . . 134

5.2 .5 Unconstrained Tests: Amplitude Variation . . . . . . . . . . . . 142

5.2 .6 Unconstrained Tests: Skin Stiffness . . . . . . . . . . . . . . . 146

5.2 .7 Chapter Summary . . . . . . . . . . . . . . . . . 150

$\begin{array}{lll}6 & \text { Conclusions } & 152\end{array}$

6.1 Summary of Contributions . . . . . . . . . . . . . . . . . . . 153

6.2 Future Work . . . . . . . . . . . . . . . . . . . . . . . . . . 160

\begin{tabular}{ll}
\hline Bibliography & 167
\end{tabular} 


\section{List of Figures}

$3.1 \quad$ Active bending of a planar active tensegrity beam . . . . . . . . . . . 31

3.2 Tip load applied to planar tensegrity beam . . . . . . . . . . . . . . 33

$3.3 \quad$ Static tensegrity beam used in load-displacement experiments . . . . 36

3.4 Detail of static tensegrity beam joint construction . . . . . . . . . . . 38

3.5 Stress-strain curve of braided cable . . . . . . . . . . . . . . . . . . . 39

3.6 Mounting base for static tensegrity beam . . . . . . . . . . . . . . . . 40

3.7 Tensegrity beam loading for displacement tests . . . . . . . . . . . . . 43

3.8 Tracking tip displacement of the static tensegrity beam . . . . . . . . 44

3.9 Displacement of base node axle . . . . . . . . . . . . . . . . 45

3.10 Rigid-body rotation of beam due to base node displacement . . . . . 45

3.11 Load-displacement plot of base node . . . . . . . . . . . . . . 46

3.12 Tensegrity beam deforming out-of-plane under load . . . . . . . . . . 47

3.13 Standard deviations of beam tip displacement data . . . . . . . . . . 48

3.14 Load-displacement plot of static tensegrity beam tip . . . . . . . . . . 49

3.15 Force density plot of tensegrity beam cables . . . . . . . . . . . . . 51

4.1 Actuation of the tensegrity planar cross unit cell. . . . . . . . . . . . 54

4.2 Geometry of the unit cell to relate cable lengths . . . . . . . . . . . . . . 56

4.3 Strain of release and contraction cables . . . . . . . . . . . . . . . . . 59

4.4 Strain from actuation delta compared to prestress reorientation . . . 60

4.5 Schematic of cam drum concept . . . . . . . . . . . . . . . . 62

4.6 Minimum error for cam shape parameter $\bar{m}$. . . . . . . . . . . . . 66

4.7 Actuation delta error along the cam drum . . . . . . . . . . . . . . . . 67

4.8 Actuations caused by each side of the cam drum . . . . . . . . . . . . 68

4.9 Cam shape for actuation drum . . . . . . . . . . . . . . . . . 69

4.10 Optimum $\bar{m}$ and error for different drum radii . . . . . . . . . . . . . 70

4.11 Triangular fin planform of a spotted eagle . . . . . . . . . . . . . . . 74

4.12 Foil shape of a dissected cownose ray . . . . . . . . . . . . . . . . 75

4.13 Schematic of fin planform with beam position . . . . . . . . . . . 76

4.14 Schematic of fluid loading model . . . . . . . . . . . . . . . . . . . . . 79

4.15 Determination of fluid loading at a span-wise location . . . . . . . . . . 81

4.16 Fluid loading distribution on the triangular fin planform . . . . . . . 82

4.17 3D model of fin shape $\ldots \ldots \ldots$. . . . . . . . . . . . . . . . . . . . . . . . . . . . 85

4.18 Tensegrity beam actuation system . . . . . . . . . . . . . . . . . . 87

4.19 Active tensegrity beam node detail . . . . . . . . . . . . . . . . . . . 88 
4.20 Cable tensioning mechanism for active tensegrity beam . . . . . . . . 90

4.21 Assembled active tensegrity beam . . . . . . . . . . . . . . . . 91

4.22 Acrylic mold for artificial skins . . . . . . . . . . . . . . . . . . . . . 92

4.23 Assembled tensegrity beam fin . . . . . . . . . . . . . . . . . . . . 93

4.24 Active tensegrity beam displaying bending amplitudes . . . . . . . . . 96

4.25 Beam amplitudes as a function of input actuation . . . . . . . . . . . 97

4.26 Measurement of fin amplitude . . . . . . . . . . . . . . . . . . 98

4.27 Fin amplitude as a function of input actuation . . . . . . . . . . . . . . 99

5.1 Test rig for hydrodynamic experiments . . . . . . . . . . . . . . . . . 104

5.2 Load cell attachment schematic . . . . . . . . . . . . . . . . . . 105

5.3 Schematic of constrained and unconstrained experiments . . . . . . . 109

5.4 Orientations for air power measurements . . . . . . . . . . . . . . . 110

5.5 Measurement of fin tip amplitude in water . . . . . . . . . . . . . . . 115

$5.6 \quad$ Fin tip amplitudes with and without surface wave suppression. . . . . 117

5.7 Fin tip amplitudes over a range of flow speeds. . . . . . . . . . . . . . 118

5.8 Instantaneous power measurments . . . . . . . . . . . . . . . . . . . . 120

5.9 Air power, gross power, and net power . . . . . . . . . . . . . . . . . 122

5.10 Instantaneous thrust for all flapping frequencies . . . . . . . . . . . . 122

5.11 Frequency components of thrust measurements. . . . . . . . . . . . . 123

5.12 Net thrust produced with and without wave suppression plate. . . . . 124

5.13 Propulsive efficiency with and without wave suppression plate. . . . . 125

5.14 Propulsive efficiency with and without wave suppression plate, plotted against Strouhal number. . . . . . . . . . . . . . . . . 126

5.15 Net power for a range of flow speeds. . . . . . . . . . . . . . . . . . . 127

5.16 Coefficient of power for a range of flow speeds. . . . . . . . . . . . . . 128

5.17 Net thrust for a range of flow speeds. . . . . . . . . . . . . . . . . . . 129

5.18 Coefficient of thrust for a range of flow speeds. . . . . . . . . . . . . . 130

5.19 Propulsive efficiency results for a range of flow speeds. . . . . . . . . . 131

5.20 Net power for unconstrained experiments . . . . . . . . . . . . . . 135

5.21 Coefficient of power for unconstrained experiments . . . . . . . . . 136

5.22 Instantaneous fin position along test rail in free swimming . . . . . . 136

5.23 Free-swimming velocity in unconstrained experiments . . . . . . . . . 137

5.24 Fin stride length in free-swimming . . . . . . . . . . . . . . . . . 138

5.25 Free-swimming economy plotted against frequency and velocity . . . 139

5.26 Strouhal number in free-swimming tests . . . . . . . . . . . . . . . 141

5.27 Free-swimming economy versus Strouhal number. . . . . . . . . . . . 142

5.28 Free-swimming velocities after accelerating from rest . . . . . . . . . 143

5.29 Free-swimming velocity for three fin amplitudes . . . . . . . . . . . . 144

5.30 Net power in free swimming for three fin amplitudes . . . . . . . . . . . 144

5.31 Strouhal number in free swimming for three amplitudes . . . . . . . . 145

5.32 Free-swimming economy for three amplitudes . . . . . . . . . . . . . 146

5.33 Free-swimming velocity for both soft and stiff skins . . . . . . . . . . 147

5.34 Free-swimming economy for both soft and stiff skins . . . . . . . . . . 148

5.35 Passive deformations of soft and stiff skins . . . . . . . . . . . . . . . 149 


\section{Nomenclature}

$\bar{\Delta}_{\text {act }}$ Actuation delta for large-amplitude deformations of a tensegrity planar cross unit cell

$\bar{P} \quad$ Cycle-averaged power

$\bar{s} \quad$ Normalized arc length swept through by cam-shaped actuation drum

$\bar{T} \quad$ Cycle-averaged thrust

$\Delta T_{j} \quad$ Change in cable tension in the $j$ th cell due to external tip load

$\delta_{a}^{*} \quad$ Additional actuation release to account for prestress reorientation

$\delta_{\mathrm{a}, j} \quad$ Input actuation for tensegrity cable, in the $j$ th cell

$\eta_{\mathrm{F}} \quad$ Froude propulsive efficiency

$\lambda \quad$ Force density in cable elements

$\Pi_{i} \quad$ Force per unit span

$\sigma_{\eta_{\mathrm{F}}} \quad$ Standard deviation of Froude propulsive efficiency

$\sigma_{\xi} \quad$ Standard deviation of free-swimming economy

$\sigma_{S t} \quad$ Standard deviation of Strouhal number

$\xi \quad$ Free-swimming economy

A Tip amplitude of tensegrity beam or fin tip, based on context

$A_{\text {corr }}$ Correction for tensegrity beam tip displacement due to movement of base node

$A_{\mathrm{c}} \quad$ Cross-sectional area of cable structural element

$A_{P} \quad$ Amplitude of tensegrity beam deformation due to external load $P$

$A R_{\text {cell }}$ Aspect ratio of tensegrity unit cell

$A R_{\text {fin }}$ Fin aspect ratio

$c_{d} \quad$ Coefficient of drag 
$C_{T} \quad$ Coefficient of thrust

E Modulus of elasticity

$f \quad$ Flapping frequency

$F_{\Delta} \quad$ Resistance force arising from geometric actuation delta

$F_{\text {pr }} \quad$ Resistance force arising from prestress reorientation

$F_{\text {plate }}$ Total normal force on the fin

$h \quad$ Height of tensegrity beam

$k \quad$ Axial stiffness

$K_{\text {bend }}$ First-order bending stiffness for a multiple-cable tensegrity beam

$k_{\mathrm{r}} \quad$ Relative stiffness

$L \quad$ Total length of tensegrity beam

$l \quad$ Element length

$L_{0} \quad$ Original length of tensegrity unit cell in neutral position

$L_{\text {con }} \quad$ Length of contracting cable

$L_{\text {rel }} \quad$ Length of releasing cable

$N \quad$ Number of cells in tensegrity beam

$P \quad$ External load acting on tensegrity beam tip

$P_{\text {cr }} \quad$ Critical slackening load

$S \quad$ Fin planform area

$s \quad$ Arc length along cam

St Strouhal number

$T_{\text {opt }} \quad$ Optimal cable pretension

$T_{0, j} \quad$ Cable pretension in the $j$ th cell of a MC tensegrity beam

$U \quad$ Fluid flow speed

MC Multiple Cable-routed clustered actuation 


\section{Chapter 1}

\section{Introduction}

Unmanned underwater vehicles (UUVs) have emerged in the last several decades as powerful tools for ocean researchers, several industries, and the military, allowing them to carry out mapping, surveying, monitoring, and inspection tasks for durations much longer than would be possible with a manned submersible, and at lower costs [1]. The vast majority of contemporary UUVs have rigid hulls and utilize rotary propellers for both thrust and maneuvering. However, standard propellers have several drawbacks including undesirable noise, the propensity for entanglement, peak efficiencies only within a narrow operational band, and limited accelerations. The result is that current UUV designs are limited in terms of stealth, operating environments, efficiency, and maneuverability.

Fortunately, biology provides a source of inspiration for the next generation of UUVs that have the potential to outperform their predecessors in each area of weakness identified above. A plethora of designs for underwater vehicles can be drawn from the oceans, where animals have been tuned by natural selection over millions of years to fill ecological niches; this process has resulted in some swimmers that are highly maneuverable and efficient. Broadly, there is increasing interest towards biologically-inspired ("bio-inspired") designs in order to leverage the advantages of flexible-bodied organisms using unsteady flapping propulsion [2]. Furthermore, the 
U.S. Navy has specifically expressed interest in bio-inspired designs in order to create engineered systems that can meet or exceed the performance seen in biological swimmers [3].

The manta ray (Manta birostris) has recently been identified as an ideal candidate for the design of an efficient and highly maneuverable biologically-inspired underwater vehicle [4]. Manta rays use the complex motions of their broad, enlarged pectoral fins for both propulsion and maneuvering. It is clear from observation that manta rays (and several other species of batoid rays) are excellent swimmers, exhibiting high endurance, efficient cruising, large speed bursts, high turning rates, small turning radii, station keeping in currents, and silent, stealthy propulsion — exactly the types of qualities that are desirable for a bio-inspired UUV. Noting these qualities, the overarching goal of this work is to study batoid ray propulsion as a model for an engineered system with similar performance capabilities.

Towards this overarching goal of understanding and utilizing batoid ray propulsion, there are essentially two main challenges to overcome. First, the swimming performance of batoid rays is not well quantified. Only superficial observations such as speed, flapping frequency, turning rates, and general descriptions of fin kinematics are available, and direct measurements of thrust and power in the freely swimming animals do not exist. With this lack of information, it is difficult to estimate how efficient or economical their swimming really is. Moreover, the design of a vehicle propulsion system is impractical without first knowing its thrust production capabilities and power requirements.

Second, the kinematic displacement fields of batoid ray fins are complex, making it challenging to reproduce the fin motions with an engineered system. If the ultimate goal is to produce a vehicle with a ray-like propulsion system, then the subject of physically recreating the complex fin kinematics is non-trivial. Additionally, it is unclear which kinematic components relate most strongly to swimming performance. Much is still unknown about the unsteady hydrodynamics of flapping propulsion, but 
it is assumed here that reproducing the kinematics of ray fins will result in similar hydrodynamic performance, in terms of thrust production and power input to the fluid.

This proposed research therefore has multiple specific areas of study to address some of the challenges related to understanding batoid ray propulsion:

\section{- Quantify the swimming performance of an artificial, batoid-inspired} fin.

In order to understand how an artificial fin should be operated to maximize its performance, specific measurements must be taken. A ray-like, robotic fin allows for the direct measurement of net thrust (in constrained tests) and swimming speed (in unconstrained tests) as they relate to kinematic parameters (specifically, frequency and amplitude of flapping). When mechanical power input is measured as well, propulsive efficiency and free-swimming economy can be calculated, indicating the level of performance that is possible using an artificial ray fin. These measures are useful for determining the viability of a ray-like UUV, guiding the design of future artificial fins, and additionally lend insight to observed biological swimming patterns.

- Create a tensegrity-based fin that reproduces the major kinematics of batoid rays.

In order to experimentally measure the performance of batoid swimming, an artificial robotic fin, which is capable of reproducing the major kinematic components seen in biology, must be designed and fabricated. Robotic fins allow for the creation of prescribed, repeatable kinematics that closely resemble biological fins, and thereby enable the direct measurement of ray-like swimming performance. A small number of recent experimental studies have used robotic fins to produce some of the kinematic components of ray fins, but it appears that there are currently no studies that fully quantify the swimming perfor- 
mance of a robotic fin with both large span-wise bending and a chord-wise traveling wave. Active tensegrity structures have recently been proposed as a means of recreating the kinematics of batoid rays [5], but this solution has yet to be experimentally verified or implemented as a fin. Developing robust fabrication and actuation methods for active tensegrity beams represents an important contribution that enables the creation of a ray-like fin.

\section{- Verify experimentally the mechanics of tensegrity beams.}

In order for tensegrity beams to be implemented as the structural basis for a robotic fin, their mechanical response to external loading must be verified experimentally. Active tensegrity beams are advantageous to use in a robotic fin because they are capable of large amplitude deformations at a low energetic cost, can have a high stiffness for relatively little mass (high structural efficiency), and allow for the migration of actuators outside the structure. The loading response of planar tensegrity beams with an optimal cable routing scheme has previously been investigated both analytically and in numerical simulations, however the loading response of these specific structures has not yet been verified experimentally. Performing structural loading tests to verify the response of these beams directly enables their design and use in a robotic fin, with confidence that their kinematics will be predictable even when subject to hydrodynamic loading.

The pursuit of these goals is documented in this dissertation, which is laid out as follows. Chapter 2 gives an overview of current UUV technology and its limitations, covers relevant information on the biology of batoid rays, reviews research on the experimental hydrodynamics of unsteady flapping propulsion, looks at the state of the art in biologically-inspired propulsors (including other attempts at ray-like fins),

and gives an introduction to tensegrity, the structural approach used here to recreate ray fin motions. Chapter 3 gives an overview of the design and mechanics of planar 
active tensegrity beams, and then shows an experimental validation of the loading mechanics using a beam with an optimal cable routing strategy. Chapter 4 describes how batoid ray kinematics are recreated in this work, from the structural basis of active tensegrity to the final implementation as a robotic fin. Chapter 5 explains the hydrodynamic test setup for this robotic fin and presents swimming performance results for both constrained efficiency and unconstrained free-swimming economy experiments. Finally, chapter 6 summarizes the major results, gives conclusions drawn from this work, and indicates fertile areas of research which could follow. 


\section{Chapter 2}

\section{Background}

A study of biologically-inspired propulsion for use in underwater vehicles naturally spans several areas of research, including biology, fluid mechanics, structural mechanics, and robotics. A review is given of relevant information relating to underwater vehicles in general and specifically to biologically-inspired ones. Pertinent details about the biology of batoid rays are given, and related studies on the fluid mechanics of flapping propulsion are described. Finally, a brief introduction to tensegrity structures is presented, in order to lay the groundwork for this study's contributions.

\subsection{Underwater Vehicle Technology}

Underwater vehicles can be generally classified by whether or not they contain human occupants, who perform onboard functions such as navigation and control [6]. Those which do are either submarines, used principally for military purposes [7], or submersibles, used principally for scientific, industrial, and exploratory purposes [8]. Major limitations of manned vehicles are that they need to be large enough to accommodate human occupants, and require multiple systems to support human life underwater.

Unmanned vehicles are free from these requirements and therefore can be smaller, 
somewhat less complicated, and have extended mission times. Vehicles without human occupants are classified most generally as unmanned underwater vehicles (UUVs), sometimes called unmanned undersea vehicles [9]. This broad category can be further broken down into either autonomous underwater vehicles (AUVs) or remotely operated vehicles (ROVs) [10]. ROVs have human pilots who operate the vehicles to accomplish specific tasks. ROVs are typically tethered, due to power and bandwidth limitations of underwater wireless communication, which obviously limits their range and presents the possibility of the tether becoming entangled [1]. AUVs, as the name implies, operate autonomously, with little to no human guidance. These vehicles typically have arrays of sensors to give navigation cues and collect data for later analysis. Because they are untethered, their range may be great, reaching depths of up to $6000 \mathrm{~m}$ [11] and even crossing entire oceans unassisted [12].

A number of reviews of existing UUV technology are available [1, 2, 6], and they reflect that UUVs typically fall into one of two categories based on general shape and function [10]: many long range AUVs have a streamlined "torpedo-like" shape that function well for low-drag, efficient cruising; meanwhile, numerous ROVs exhibit a "box design" that is optimized for low-speed maneuvering. A tradeoff is presented by these designs: streamlined UUVs suffer from poor low-speed maneuverability while UUV designs with low-speed maneuverability are either incapable of or are inefficient at high-speed cruising. Each type of vehicle works well for specific applications, but a vehicle combining the strengths of both would be desirable [13].

The limited turning ability of traditional streamlined AUVs is often cited as a major drawback of this general design, with the best reported turning radius being estimated as around four body lengths (also noting that the rate of turning is much slower than biological swimmers) [13]. It has been estimated that turning radius of conventional AUVs is at least 10 times larger than the corresponding value for fish [2].

Both types of UUVs predominantly use standard rotating propellers to produce thrust [14]. While traditional propellers can perform well in producing high-speed 
steady-state thrust, flapping fins have several advantages such as lower noise and reduced risk of tangling, and are even reported to have higher thrust-power ratios [15]. The propellers used on traditional UUVs have efficiencies ranging from as low as $40 \%$ [16] to as high as 70\% [17]. Meanwhile, oscillating biological propulsors have been estimated to have efficiencies as high as 90\% [17, 18]. Additionally, the performance of rigid propellers is limited to a narrow operational range where their efficiency is maximal, while flexible appendages of swimming animals maintain a high efficiency over a broad operational range [19].

Overall, aquatic animals have been observed to be much more capable than traditional UUVs in several areas: small turning radii, sudden accelerations (burst speeds), and hovering (also called "station keeping") [2]. The performance gap between UUVs using conventional designs and biological swimmers seems to be due (at least in part) to the lack of several mechanisms found in biology, such as high-lift, unsteady hydrodynamics, and the use of structural compliance. Engineered platforms typically depend on steady flows and avoid flow separation, while fish rely on unsteady flows that generate forces by shedding vortices, thus utilizing flow separation. With these differences in mind, it seems that understanding how biological swimmers produce thrust could lead to performance improvements in man-made UUVs, closing the performance gaps that exist between conventional underwater technology and what has been observed in biology.

Many types of biologically-inspired AUVs (BAUVs [20]) using various propulsion mechanisms have already been proposed and tested, including jet-based (squid [10], jellyfish [21]), caudal fin-based (tuna [22, 23], salmon [24], pike [25], and general carangiform [26]), and pectoral fin-based (sunfish [27, 28] and penguin/turtle [29]). Reviews of many other BAUVs are given by Roper et al. [11, Low [30, Kato [31] and Bandyopadhyay [2]. In these studies, it is emphasized that biologically-inspired underwater vehicles hold the potential for improving both energy efficiency and maneuverability compared to conventional underwater vehicles. Progress towards this 
goal is discussed in the following section, where several types of flapping propulsors and freely swminng BAUVs are reviewed, especially efforts related to ray-like platforms. But first it is important to understand the swimming style of real rays, and therefore a review of relevant biological information is given.

\subsection{Taking Inspiration from Biology}

The performance gap between current UUV technology and biological swimming can be narrowed by further study of unsteady propulsion and application of our understanding to the next generation of UUVs. As Bandyopadhyay explains [2]: "The goal is not to build a robotic replica of animals. The goal is to distill the science from biology and implement that on existing platform components with a minimum of logistical and a maximum of operational impact." This section covers previous attempts at understanding and recreating the performance of biology, in order to identify constructive areas of research for this work and future studies.

\subsubsection{Biological Observations}

Aquatic organisms exhibit an enormous array of locomotory styles across a wide range of size scales, from the flagella of bacteria to the tails of whales [32]. While interesting and complex, low Reynolds number flows are dominated by viscosity and are typically found on small size scales [33, 34], so they will not be discussed here. Instead the focus is on swimming animals of moderate body size operating at intermediate to high Reynolds numbers, which are dominated by inertial fluid forces. With the exception of jetters (e.g. squid) and rowers (e.g. a duck's feet when swimming on the surface of water), most animals in this regime use a lift-based mechanism for thrust production [13, 31, 33, 35]. This process generally involves the motion of either appendages or the entire body (or both) to create and control vortices [16, 36 38]. Using oscillatory movements, these swimmers repeatedly form and shed starting and 
stopping vortices at each reversal, creating high levels of lift during each stroke, which is angled forward to produce thrust. This lift-based locomotion is believed to be up to $90 \%$ efficient in some cases [17, 18].

Although many types of biological swimmers using this general locomotory scheme are worthy of investigation, this study is inspired by the manta ray. They have recently been identified as having a number of desirable characteristics that should be emulated in a next generation UUV [4, 5, 20, 39,48]. These include: swimming over very large distances at impressive sustained speeds, high maneuverability, accelerating quickly to reach high burst speeds, proficiency at station keeping in currents, having low flapping frequencies compared to many other fish species (reducing the possibly of noise generation), and importantly, are regarded as very efficient swimmers. Additionally, the overall shape of manta rays makes them good candidates for a UUV platform because their central bodies are approximately rigid (allowing for easier placement of equipment in a hull, compared to flexible-bodied fish) and the broad, flat shape allows for the possibility of low energy, long distance gliding using a buoyancy drive [49].

Limited research has been done on manta rays, with most work relating to their overall size, structure, population dynamics, behavioral patterns and migrations [5053. Unfortunately, even less information is available specifically relating to their swimming performance. However the smaller and more common cousins of manta rays in the family myliobatoidea (including eagle rays, cownose rays, and mobula rays) use a similar locomotor style and can act as stand-ins for gaining information about this type of swimming. This family of rays comes in a wide range of sizes, suggesting that the locomotor strategy is highly scalable, so that UUV platforms of nearly any desired size could be created.

Members of the myliobatoidea family are more generally classified as batoid rays, which are a superorder of dorsoventrally flattened cartilaginous fish, closely related to sharks and are found in every ocean of the world [54, 55]. Although some ba- 
toid species utilize axial undulations of the body (like most sharks), the morphology of most batoid species is characterized by diminished tails and by greatly enlarged pectoral fins, which provide both thrust and maneuvering. These median paired fin (MPF) 56] species are the subject of interest for the current study and axial undulators are excluded, so that the kinematics responsible for swimming can be isolated to only the fins.

The MPF swimming styles of batoids can be broken down into two major groups: rajiform, a drag-based swimming style in which the fins undulate, and mobuliform, a lift-based swimming style in which the fins oscillate [56, 57]. The distinction between these undulatory and oscillatory swimming modes is drawn from the observed number of chord-wise waves present on the fin, with undulators having more than one full wave present and oscillators having less than half a wave present ${ }^{1}$ [54]. The largeamplitude oscillatory swimming style is often described as appearing much like the flapping of bird flight [54, 58]. Both swimming modes feature curvature of the fins in the chord-wise and span-wise directions, but the undulatory mode is dominated by chord-wise curvature and the oscillatory mode is dominated by span-wise curvature.

Schaefer and Summers [55] compared the morphology of cartilaginous fin skeletal structures for both oscillating and undulating rays. Both groups have a similar structure in general, which is characterized by a series of fin radials extending outward from the pectoral girdle, with each fin radial comprised of multiple cylindrical cartilage elements stacked end-to-end. Muscles on both the dorsal (upper) and ventral (lower) side of the fins alternately contract to flex the fin radials up and down, resulting the overall observed locomotion patterns for each species. Schaefer and Summers found that specific differences in skeletal morphologies strongly predicted the locomotory style of swimming. Notably, oscillators were found to have a pattern of cross-bracing between radials in medial areas, effectively stiffening the fins there, whereas cross-bracing was absent in most undulators. Their findings suggest that a

\footnotetext{
${ }^{1}$ Species showing between half and a full wave present on the fin are sometimes called "semioscillatory" [55].
} 
somewhat stiffer, less flexible fin structure may be beneficial for oscillatory swimming.

Parson, Fish, and Nicastro [59] compared the maneuverability of oscillators and undulators by measuring the velocities, turning radii and turning rates for individuals of each group in a large aquarium. It was found that the minimum turning radii of both groups were comparable, at about 0.9 body lengths (BL). However, oscillatory rays had a higher maximum turning rate and velocity $\left(48^{\circ} / \mathrm{s}\right.$ and $\left.1.1 \mathrm{BL} / \mathrm{s}\right)$ compared to undulators (at $32^{\circ} / \mathrm{s}$ and $0.8 \mathrm{BL} / \mathrm{s}$ ). Parson et al. note that oscillatory rays have been observed performing essentially zero-radius turns from a resting position and so the measurements of uncoerced motions from this study may not represent maximum potential turning performance. By these specific performance measures though, it appears the oscillatory swimming style is more desirable to emulate for an underwater vehicle. Additionally, it is thought that lift-based oscillatory swimming should be more efficient at higher cruising speeds [32] and generally a lift-based oscillatory mode should produce larger and more efficient thrust compared to a drag-based mode [60].

Rosenberger [54] compared the pectoral fin kinematics of several batoid species with similar but distinct locomotor patterns. It was found that rather than a clear division between purely undulatory (rajiform) or oscillatory (mobuliform) locomotion, there is instead a continuum of swimming styles represented across the species. Some species, such as the butterfly ray (Gymnura micrura), were even found to shift between swimming modes depending on distance from the bottom surface of the tank - exhibiting undulatory locomotion close to the bottom and oscillatory locomotion away from the bottom. This interesting behavior draws attention to an even broader trend across batoid species: benthic species (near the substratum in a body of water) are generally undulatory swimmers whereas pelagic species (in the open water column) are generally oscillatory swimmers. Rosenberger notes that while undulators are better at low-speed efficiency and maneuverability, oscillators excel at efficient steady cruising and thrust production. Since efficient steady cruising in open water has been identified as a desirable characteristic for UUVs, oscillatory locomotion is 
further justified as the swimming mode of interest for the current study.

In comparing the various batoid species, Rosenberger found that the means by which swimming speed is modulated depends on swimming style. For oscillators, swimming speed is most influenced by wavespeed (the stream-wise speed of the partial wave present on the fin) and fin tip speed, while keeping frequency and amplitude relatively constant over the range of speeds observed. Of all species studied, the cownose ray (Rhinoptera bonasus) was the most oscillatory, with a wavenumber of only 0.4 . The oscillatory flapping of the cownose is characterized by a relatively low frequency $(1 \mathrm{~Hz})$ and high amplitude (35\% of total wingspan $\left.{ }^{2}\right)$, both of which are typical for pelagic oscillators. It is noted that the cownose ray has a somewhat unusual asymmetric flapping pattern, with the fins staying mostly above the dorsoventral plane. This flapping pattern is postulated to derive from the dependence of the cownose on a benthic diet, despite that it spends much of the time in the pelagic zone. Other pelagic oscillators that do not depend on a benthic diet generally have a more symmetric flapping pattern.

Heine [58] carried out what is probably the most in-depth biological study of oscillatory batoid ray swimming kinematics, focusing on the cownose ray and also the bullnose eagle ray (Myliobatis freminvillei). The kinematics of the pectoral fins and the body overall were tracked while specimens swam in test tanks. Heine's data shows that the vertical amplitude of fin flapping motions are approximately smooth sinusoidal waves. Horizontal velocity was highly uniform, with no noticeable oscillation associated with flapping, and the swimming velocities ranged from 0.3 to $0.9 \mathrm{~m} / \mathrm{s}$ (approximately 1 to $3 \mathrm{BL} / \mathrm{s}$ ). In examining the relationship between various kinematic parameters and swimming speed, there was a weak correlation between flapping frequency and swimming speed, with most flapping frequencies around $1 \mathrm{~Hz}$. An even weaker correlation was found between flapping amplitude and swimming

\footnotetext{
${ }^{2}$ This is equivalent to a fin tip displacement of approximately $88 \%$ of a single fin's span. However a completely symmetric flapping pattern would sweep through the same distance with an amplitude of $44 \%$ of a single fin's span.
} 
speed, with flapping amplitudes (normalized by half the wing span) in the range of $0.4-0.9$. Again, cownose rays have an asymmetric flapping pattern, so a symmetric pattern would sweep through the same distance using an amplitude of $0.2-0.45$ (also nondimensionalized by the span of a single fin).

The kinematic parameter that correlated most strongly to swimming speed was the fin tip speed (specifically on the upstroke). Heine notes that many other types of fish have higher flapping speeds relative to their forward speed, and that this frequency is generally correlated with swimming speed. However these oscillatory rays show relatively slow flapping compared to their forward speed, with no correlation to flapping frequency. It appears this fin tip speed is modulated by changing the duration of time spent at the maximum amplitude, slightly shifting away from a purely sinusoidal waveform.

Heine tracked the velocity of several points along the span of the fin (from the posterior direction) and also tracked the twist angle along the span. The measured twist angle at $60 \%$ of the wing span was up to $20^{\circ}$ and importantly, this twist maintained a phase lead over the heaving motion. It was found that there is some correlation between maximum twist angle and swimming speed. The angle of attack was then calculated at various points, and not surprisingly it increased along the span (since the heave component of the local velocity increases along the span). The maximum angle of attack near the most distal portion of the fin was about $25^{\circ}$.

Additionally, Heine experimentally found force coefficients for sections of the fins and body by testing rigid castings in steady flow at various angles of attack. Combining the resulting force coefficients with the kinematic data, his calculations show that the average net thrust from the fins is about $0.1 \mathrm{~N}$, and no greater than $0.4 \mathrm{~N}$. From this it was concluded that the thrust produced by the fins is far too small to propel these rays forward. Instead it is proposed that thrust is produced by the oscillation of the ray's rigid body, caused by the heaving of the fins. Potential flow theory applied to this small amplitude body oscillation predicted thrust to be about $0.75 \mathrm{~N}$, however 
experimental attempts to measure it resulted in a net thrust from the body of only $0.04 \mathrm{~N}$.

While the quality of Heine's data seems to be good, it appears two major errors were made in coming to his conclusion about thrust production from the body and not the fins directly. First, Heine himself admits that a quasi-steady assumption is probably not valid for determining the instantaneous forces on the fin which derive from large amplitude flapping. Indeed, it should be expected that the forces found in steady flow are not the same as those found from unsteady flow of a high amplitude pitching/heaving motion. There are several effects relevant to unsteady flows that would not be present in steady flows such as acceleration reactions (the force required to accelerate a mass of fluid near the fin), the Wagner effect (the delay in formation of bound circulation about the fin, since the direction of circulation changes every half-cycle), and vortex shedding dynamics.

Second, it seems that any change in fin shape (such as the camber of a given section along the span) throughout the flapping cycle, whether from active muscular contraction or from passive flexibility, has been completely disregarded in the process of finding steady force coefficients on the rigid fin sections. It is obvious that ray fins are highly flexible, a point that is even reinforced by some of Heine's own observations, and do indeed change shape throughout the flapping cycle, so this provides further doubt that the calculated net force accurately represents real ray fins.

For these reasons, it will be assumed that Heine's conclusion about the lack of thrust from the fins is incorrect ${ }^{3}$, Despite this shortcoming, Heine's work provides many valuable observations about oscillatory ray swimming overall, and there may be some credence to the idea that an oscillating body can contribute to thrust production.

While biological studies inform us about the swimming styles, fin kinematics, and performance capabilities of rays, there is a limit to their usefulness for designing artificial propulsors because large variations can exist amongst both species and in-

\footnotetext{
${ }^{3}$ This is an important point, because there would be no purpose in further study of an isolated fin if Heine's conclusion was correct.
} 
dividuals within a species, making it very difficult to isolate individual components that contribute to swimming performance [61]. Additionally, observations of animals in the wild or of specimens in a laboratory may not represent maximum performance.

\subsubsection{Biologically-Inspired Propulsors}

Lauder [61] notes that in recent years swimming hydrodynamics has garnered attention from both biologists and engineers, but several key issues need to be addressed in order to fully understand biological locomotion. Both the structure and deformations/motions of propulsive surfaces need to be studied in three dimensions, since many biological motions are complex and cannot be represented by only twodimensional analyses. Additionally, much is still unknown about the flexibility of biological appendages and the effects of flexibility on performance. Moreover, even less is known about the ability of animals to change appendage flexibility during swimming. Lastly, the extent to which propulsor deformations are either passive (as a result of fluid forces) or actively controlled is still largely unknown.

Robotic test platforms are one strategy that Lauder suggests for addressing the issues above. Robotic systems offer the advantage of an extremely high level of control compared to working with biological test subjects: motions can be accurately repeated and varied within the range of biological observations and beyond. Structural changes, such as variations in planform or stiffness, can also be studied relatively easily. Additionally, certain measurements that are difficult to make on biological subjects, such as direct force measurements, can be carried out routinely on robotic systems. Lauder indicates that propulsor deformation is one area where the use of robotics can be extremely useful, because the deformation of flexible robotic appendages would allow for the study of both performance measures and wake dynamics. It is emphasized that robotic test platforms should be self-propelling, swimming against an oncoming flow by generating their own propulsive forces.

Some of the most canonical experimental studies of flapping propulsion are based 
on idealizing the problem to that of a two-dimensional pitching, heaving, or combined pitching/heaving airfoil [62]. Triantafyllou, Triantafyllou, and Grosenbaugh 63] found that rigid oscillating foils produce net thrust for certain combinations of parameters and that the thrust production is directly related to a vortex pattern formed in the wake of the foil. The wake contains a staggered array of vortices with rotational directions reversed compared to a standard von Kármán vortex street, indicating thrust instead of drag, and averaged over time, appears as a convectively unstable jet flow. Triantafyllou et al. found that the wake was amplified within a relatively narrow frequency band, and importantly, that the maximum wake amplification is correlated with maximum foil efficiency.

The hydromechanical efficiency used here is the one commonly defined as Froude propulsive efficiency, $\eta$, which is a ratio of useful power output (typically the product of thrust and swimming velocity) to net power input to the fluid [18]. Oscillating frequencies in swimming propulsion are nondimensionalized as the Strouhal number, $S t$, which is the product of vortex shedding frequency and wake amplitude, divided by flow speed. This nondimensional frequency essentially quantifies how often vortices are shed, and how closely they are spaced together ${ }^{4}[16]$.

Triantafyllou et al. found in their experimental work a maximum efficiency of about $20 \%$ at a Strouhal number of about 0.25 (although kinematic parameters were not optimized to increase maximum efficiency). They note that the highest efficiencies occur in a range from $S t=0.25$ to 0.35 , which coincides with the range of Strouhal numbers observed for a wide variety of swimming animals, concluding that animals swim in this range to achieve maximum efficiency. Taylor, Nudds, and Thomas 64] reinforce this idea, noting that it applies not only to swimming, but to the cruising flight of birds, bats, and insects as well. They expand the optimal Strouhal number range to $0.2-0.4$, and posit that animals using oscillatory lift-based propulsion tune

\footnotetext{
${ }^{4}$ Dabiri 38 makes a strong argument that Strouhal number is merely a consequence of optimal vortex formation time, limited by the energetics involved, and that optimal vortex formation is a more fundamental principle.
} 
their kinematics to be within this range.

Anderson et al. [65] conducted experiments with a rigid, pitching/heaving, twodimensional, high aspect ratio foil and found parameter combinations that resulted in high efficiency, up to $87 \%$. High efficiencies were obtained by ensuring the proper Strouhal number range was satisfied $(0.25-0.4)$, that heave amplitudes were large (75\% of chord length), oscillatory pitching leads heaving by a phase of about $75^{\circ}$ and a nominal angle of attack was between $15^{\circ}$ and $25^{\circ}$. These tests were conducted at a relatively high flow velocity of about 3.6 chord lengths per second, compared to 1.7 chord lengths per second in the experiments by Triantafyllou et al. described above. The results were compared to linear and nonlinear inviscid numerical simulations and agreement was mixed: for some sets of parameters there was decent agreement, but for other sets of parameters there were large discrepancies. Disagreement was attributed to the formation of strong leading-edge vortices, which were observed with particle image velocimetry (PIV) wake visualization, and are correlated to high efficiency. This discrepancy motivates further experimental studies of flapping propulsion, because computational fluid models may not be able to capture all flow phenomena.

The idea that wake structure qualitatively changes with kinematic parameters (such as leading-edge vortex formation for large heave amplitudes, as described in Anderson et al.) was investigated previously by Koochesfahani [66]. Koochesfahani showed with dye visualizations of a low-amplitude pitching airfoil that wake structure depends heavily on frequency, amplitude, and waveform (sinusoidal versus nonsinusoidal). A variety of different wakes were produced by varying these parameters, including a classic reverse von Kármán vortex street, but also wakes with two or even three vortices of the same sign shed per half-cycle 5 Even more exotic, asymmetric wakes were produced with non-sinusoidal pitching oscillations (as are sometimes used by oscillatory batoids). Also it was found from visualizations that axial flow through the vortex cores was present, further demonstrating how complex the wake structures

\footnotetext{
${ }^{5}$ Schnipper, Andersen, and Bohr [67] present excellent visualizations of pitching foil wakes, with formations up to $8 \mathrm{P}$.
} 
of flapping foils can be, even for a relatively simple two-dimensional pitching motion.

The oscillating propulsion studies discussed above used rigid foils, while notably most swimming animals use flexible appendages [68, 69]. Some limited experimental studies have been done on flexible two-dimensional flapping foils. For example, Prempraneerach, Hover, and Triantafyllou [70] found that a pitching and heaving foil with the properly selected amount of chord-wise flexibility can have $36 \%$ higher propulsive efficiency compared to a rigid foil, with only small loss in thrust. Riggs, Bowyer, and Vincent [15] found that a flexible pitching foil with chord-wise variation in stiffness had up $26 \%$ better thrust production compared to a stiffer, non-biomimetic foil. Also the biomimetic foil showed thrust production over a greater frequency range compared to the non-biomimetic foil. Heathcote, Wang, and Gursul [71] found that a heaving, cantilevered foil benefitted in terms of efficiency from some span-wise flexibility compared to a rigid foil, but that too much flexibility was detrimental to thrust production, indicating that more flexibility is not necessarily better and that some intermediate, optimal level might exist (depending on propulsor configuration).

A two-dimensional pitching/heaving foil does generally represent the motions of a chord-wise slice through an oscillatory ray fin, however it ignores important threedimensional features of the problem: oscillatory batoid fins have amplitudes, velocities, and chord lengths that vary with span-wise location; each span-wise location oscillates along an arc, not a straight line; large amplitude flapping from span-wise curvature leads to span-wise flow on the fins, especially near the tips; and a finite aspect ratid ${ }^{6}$ fin has tip vortices, further contributing to a three-dimensional wake. Therefore to properly quantify the performance of an oscillatory batoid fin, swimming performance studies should be conducted using a fin that fully reproduces three-dimensional kinematics.

Multiple types of biologically-inspired robotic fins with fully three-dimensional kinematics have been explored [72]. Tangorra et al. [9, 73, 74] developed a propulsor

\footnotetext{
${ }^{6}$ Finite aspect ratio effects become even more important when the aspect ratio is relatively low, as it is for batoid fins [58.
} 
similar to the pectoral fin of a bluegill sunfish, having multiple independently-actuated fin rays connected with a thin membrane (a "pleated webbing"). The large number of degrees of freedom allows for many different sets of kinematics and makes this fin capable of various flapping modes. Peak instantaneous thrust on the order of $0.5 \mathrm{~N}$ was reported, and specific sets of kinematics were found to produce positive thrust throughout the flapping cycle. However no data on power input, efficiency, or other performance measures were given. Palmisano et al. [75] also developed a propulsor with fin rays, mimicking the pectoral fin of a bird wrasse fish. They present kinematic and force data, with peak instantaneous thrust on the order of $1 \mathrm{~N}$. Some large power input data is given, but this is not translated into efficiency or any other type of performance metric.

Willy and Low [76 78] developed a very low aspect ratio "ribbon fin" with a series of linkages that is intended to model the undulation of a cuttlefish or stingray. Freely-swimming velocities are reported, with top speed around one third of a chord length per second. Epstein, Colgate, and MacIver [79] developed a similar type of ribbon fin in order to reproduce kinematics of the black ghost knifefish. Experimental performance data on this fin is reported by Curet et al. [80]. Maximum average thrust was found to be on the order of $1 \mathrm{~N}$ and velocities up to one chord length per second are reported. Power measurements were not made, but efficiency is reported as wave efficiency (the ratio of undulatory wave speed to forward travel speed), which has a maximum of about 0.5 and decreases with wave frequency.

Experiments using robotic fins like the ones just described can provide interesting insight into different forms of biological swimming, but there are still very few studies of fins with kinematics similar to oscillatory batoid rays. Experimentally reproducing batoid kinematics is not a simple task and some attempts are more successful than others. For example, Brower [43] attempted to develop an artificial fin mimicking a manta ray, but due to design and fabrication issues with the actuation system, the kinematics of this fin were very limited and the fin did not self-propel in water. On 
the other hand, one of the most useful experimental studies on batoid propulsion was performed by Clark and Smits [41], who tested the thrust production of an undulatory batoid fin. Their fin allowed for the active control of low amplitude $(11 \%$ of the fin's span length) undulatory motions of varying wavelength by changing the phase between rigid rotating spars embedded in a flexible skin. Thrust and power measurements were taken by constraining the fin against oncoming flow and Froude propulsive efficiency was calculated from this setup. Both thrust and power generally increased with flapping frequency, although there was some local variation in thrust, where values decreased and then increased again with frequency. Maximum efficiency of about $54 \%$ was obtained near a Strouhal number of 0.3 . Dye flow visualizations were performed on the fin at lower Reynolds numbers and general descriptions of the complex, three-dimensional wake structures are provided. It was found that the structure of the wake qualitatively changed based on Strouhal number, and that this wake seems to be directly tied to thrust production. It is suggested that optimizing kinematics, changing planform, or introducing span-wise curvature may lead to increases in efficiency.

Dewey, Carriou, and Smits [81] investigated the wake structure of this fin more thoroughly using PIV and found that wake structure depended heavily upon both wavelength and Strouhal number. The number and coherence of vortices shed correspond to these parameters and the time-averaged momentum flux was found to form a bifurcating jet for certain sets of parameters. Maximum efficiency was associated with a non-bifurcating $2 \mathrm{~S}$ (2 single vortices shed per cycle) wake structure.

This undulatory batoid fin was also tested under free-swimming conditions in another study [40] and is one of the only known flapping propulsor studies to measure free-swimming economy (velocity divided by average power $]^{7}$. The fin was capable of freely swimming at high speeds (correlating to high frequencies), up to $2 \mathrm{BL} / \mathrm{s}$ at a $2 \mathrm{~Hz}$ driving frequency. Testing included creating a passive version of the fin, in

\footnotetext{
${ }^{7}$ Here, the power measurement used was total power, not net power to the fluid.
} 
which the two trailing actuation spars were removed so that the trailing portion of the fin was allowed to freely deform. Velocities were about $80 \%$ lower for this passive fin compared to the active version. The Strouhal number for free swimming was between 0.2 and 0.4 for most velocities ( $S t$ increased for low velocities) and was independent of wavelength or passivity. Economy generally increased with frequency for most test: 8 and the greatest economy occurred at the highest frequency and wavelength using the passive fin. The results generally demonstrate that passive flexibility might be key to maximizing the energy economy of swimming.

The most directly relevant experimental study of oscillatory batoid thrust production was performed by Moored et al. [4, 40]. A batoid-like, triangular planform fin capable of span-wise curvature (using a jointed mechanism consisting of 3 rigid plates, embedded in a flexible polymer) produced pure heaving motion, with no undulatory (chord-wise curvature) component. Thrust and power were measured at a constant flow speed across a variety of flapping frequencies in order to obtain efficiency measurements across a range of Strouhal numbers. Three other variables were considered: the amount of span-wise curvature (from flat rotation towards more biological), the amount of tip lag 9 , and the tip speed 10 , Both thrust and power were higher for the flat mode of flapping compared to the more curved mode. Efficiencies were similar for both modes, except at the lowest frequencies where the efficiency of the flat mode increased (to a maximum of just above 20\%, near a Strouhal number of 0.15), while it decreased for the curved mode. Varying tip lag had little effect for the kinematics produced in this study and swimming with tip speed modulation seemed to decrease thrust compared to sinusoidal flapping. A major implication of this study is that incorporating chord-wise flexibility in a similar fin might increase performance.

\footnotetext{
${ }^{8}$ This economy measurement showed signs of plateauing towards higher frequencies, but only decreased for one set of tests, which was the fully actuated fin with largest wavelength.

${ }^{9}$ The fin tips of oscillatory batoids are often observed to lag behind the more proximal portions of their fins.

${ }^{10}$ Since oscillatory batoids typically modulate forward speed with fin tip speed, not fin beat frequency.
} 
More recently, Russo [48] created artificial oscillatory ray fins with a high level of biological fidelity, based on a parameterized model of the ray skeletal architecture. He tested the thrust production of two fins having different inter-radial joint pattern angles and found that both a higher joint pattern angle and a higher wavenumber on the fin were correlated with increased thrust production. The results imply that specific parameters of skeletal architecture are related to swimming performance, both for biological rays and for engineered fin structures closely resembling ray skeletons. It seems that the differences in skeletal architecture lead to differences in kinematics, and that those kinematics drive swimming performance, however the specific differences between the resulting kinematics of the two fins were not quantified, leaving it unclear what types of prescribed motions might lead to improved performance (other than incorporating some amount of chord-wise traveling wave).

Aside from these studies of single fins, there are a few examples of recent attempts at creating freely swimming ray-like vehicles with paired fins [20, 82]. Several researchers interested in ray-like platforms have noted the AquaRay made by Festo, however there does not seem to be any technical work published on the vehicle [83]. A white paper available from the company describes the use of their own fluidic muscles to create span-wise curvature in the fins, and a maximum speed of about $0.8 \mathrm{BL} / \mathrm{s}$ is given, but there are no other performance metrics reported ${ }^{11}$ [84]. Love, Arroyo, and Schwartz [42] built a "Solar Ray" vehicle that was intended to swim like an oscillatory batoid while utilizing solar panels for powering the electronics. While their final prototype had a ray-like appearance, it was not able to propel itself forward at all. Wang et al. [85] built a ray-like vehicle with undulatory fins, but much of their discussion is based on control of a buoyancy system and no data is reported on swimming speeds. A few other examples of robots using undulatory locomotion are described by Low [86]. Some researchers focus on developing and integrating novel types of actuators, such as soft pneumatic actuators created by Suzumori et al. [87]

\footnotetext{
${ }^{11}$ It appears that this was a demonstration of the company's actuator technology, and not a scientific investigation of batoid propulsion.
} 
or the integration of shape memory allow (SMA) actuators by Wang et al. [88]. These studies report top speeds between 0.2 and $0.6 \mathrm{BL} / \mathrm{s}$, but no other performance measures are given. Chu et al. [89] give a review of other recent attempts at integrating novel actuator technologies into biomimetic swimming robots.

Gao et al. [44] built a fin consisting of a rigid leading edge spar that rotates at the root and is trailed by a thin membrane to create the fin shape, roughly producing ray flapping kinematics. This fin was tested alone and found to have thrust that increases with both frequency and amplitude, with a maximum thrust of about $0.85 \mathrm{~N}$. Two of these fins were attached to a body and it was reported to swim at a speed of up to $1.4 \mathrm{BL} / \mathrm{s}$, but no other performance metrics are given. Yang, Qiu, and Han [46] developed a ray-like vehicle with rotating spars attached to a thin membrane and report some free-swimming velocities as a function of amplitude, frequency, and wavenumber. The maximum velocity was about $0.4 \mathrm{BL} / \mathrm{s}$, but no other performance metrics are given. Low et al. [47, 90, 91] produced several versions of a vehicle they call "RoMan" which use rotating spars connected to a thin membrane. Various control strategies were explored and some velocity data is reported, with a top speed of $0.8 \mathrm{BL} / \mathrm{s}$, but no other performance metrics were given. Cai et al. 92 94] developed several versions of fins and vehicles imitating a cownose ray using a pneumatic muscle system. Relatively large average thrust of up to $2.75 \mathrm{~N}$ is reported for constrained tests of one of the fins and the maximum speed of one of the prototypes is $0.9 \mathrm{BL} / \mathrm{s}$. Again though, there were no other performance measures reported.

The common problem amongst all of these studies is that they do not provide data related to power consumption, and therefore important metrics such as propulsive efficiency and free-swimming economy cannot be determined. While maximum swimming speed is a decent benchmark for determining the proficiency of a new vehicle platform, it seems far more important to determine how much power is required to produce a vehicle's given speed, because ultimately this will determine the range and duration of a mission using the vehicle. 
Additionally, without efficiency or economy measurements, there is no insight into the underlying fluid mechanics of the motion. This dearth of performance data motivates further experimental work on ray-like fins that takes power consumption into consideration.

\subsection{Active Tensegrity}

The word tensegrity is a term coined by Buckminster Fuller, which is a portmanteau of "tensional integrity," reflecting the idea that these structures derive their integrity from the continuous network of tension throughout them [95]. Many different and sometimes conflicting definitions of tensegrity have been given, but generally tensegrity can be defined as [95]: "A system in a stable self-equilibriated state comprising a discontinuous set of compressed components inside a continuum of tensioned components." This definition gets at the idea that a state of self-stress holds compressive elements together using elements in tension, although it could be argued that the compressive elements need not be discontinuous (as is true of the structures used in this study).

Man-made tensegrity structures can take many forms, ranging from a balloon (a structure in self-equilibrium, composed of compressed air within a tensioned membrane) to the space frame of a geodesic dome, however the use of tensegrity as a structural foundation can actually be seen as biologically inspired. According to Ingber [96], "An astoundingly wide variety of natural systems, including carbon atoms, water molecules, proteins, viruses, cells, tissues and even humans and other living creatures, are constructed using a common form of architecture known as tensegrity." Ingber explains that tensegrities self-stabilize by redistributing stresses throughout the entire structure, giving them maximal strength for a given amount of building material and for this reason, they are found throughout nature. Using the general definition of tensegrity, the musculoskeletal systems of batoid ray fins actually are 
active tensegrity structures because muscle fibers (a network of tension elements) pull against cartilaginous radial segments (discontinuous compression elements) to produce fin motions. Of course the actual tensegrities studied here differ greatly from those found in biology, but they take advantage of some of the same principles.

In this implementation, tensegrities refer to special types of pin-jointed truss structures connected with prestressed tension elements. Here, the compression elements will be referred to as struts (rigid bars) and the tension elements will be referred to as cables (with no ability to bear compressive loads). Prestress refers to the fact that both types of elements are placed in a state of stress, even with no external loading, meaning the structures are statically indeterminate [97]. This prestress is the key to stabilizing equilibrium positions of tensegrity structures. The structures are configured such that struts and cables meet at nodes (which are generally assumed to be frictionless), and no torques are transferred through the nodes, so that loads on all elements are only axial. The class of a tensegrity structure refers to the maximum number of strut elements attached at any node [98]; so a class 1 tensegrity has no struts directly attached to one another (true discontinuous compression), and a class 2 tensegrity has at least one node with two struts connected.

Historically, tensegrity literature has focused on static structures [99], but recently there has been great interest in creating active tensegrities, in which elements (either cables or struts) are actuated to change length and thus deform the overall structure [98, 100 103]. These studies include both dynamic and quasi-static analyses. Most studies on active tensegrity are theoretical analyses and there are very few examples in the literature of researchers physically building and using active tensegrity structures, at least in part because of the complexity of their construction and actuation. For example, Aldrich, Skelton, and Kreutz-Delgado 104 describe a control methodology for tensegrity-based robots, but there is no experimental component to the study. This is in contrast to the numerous examples of static tensegrity that can be found, especially in art and architecture [97]. Several of the most prominent 
examples of physically-built active tensegrity are given below.

Pinaud, Solari, and Skelton [105] demonstrate solutions for the reconfiguration of symmetric tensegrity structures, and even show a physical model of a deployable boom that reconfigures to change its height through length change of active cables. However it does so very slowly, with no external loading, and the results of this experimental structure are not compared to predictions. Domer and Smith [106] discuss simulation and control strategies for deformation of tensegrities and validate these on a very large structure. Paul, Valero-Cuevas, and Lipson [107] showed the feasibility of producing a "walking" tensegrity robot, that took advantage of actuations developed through a genetic algorithm in simulations. Similarly, Shibata, Saijyo, and Hirai [108] developed a "crawling" tensegrity robot that uses shape memory alloy actuators. The only known example of active tensegrity being physically implemented for a swimming robot is in work conducted by Bliss et al. [109, 110], which demonstrated robust gait entrainment using central pattern generator control applied to a class 2 planar tensegrity beam (essentially acting as a caudal fin).

Recent studies by Moored lay the foundation for using active tensegrity as the structural basis for a batoid-inspired artificial pectoral fin [4, 5, 39, 111, 112]. Moored provides several important contributions towards this goal by developing: the rationale for using activity tensegrity to produce batoid kinematics; a generalized numerical model for the clustered ${ }^{12}$ mechanics of tensegrity structures; the concept of an optimal remote actuation strategy for two-dimensional (planar) beam structures, realized by cable clustering; and analytical solutions for the actuation and loading response of two-dimensional beam structures. Multiple methods for creating batoid kinematics are explored, including fully three-dimensional deformable plate structures. However the mechanics, optimization, construction, and actuation of such structures are highly complex compared to two-dimensional beams, which are suggested as a simpler strategy for physically implementing in an artificial fin.

\footnotetext{
${ }^{12}$ Moored defines clustering as the combination of several individual cable elements into one continuous cable.
} 
The analytical solutions developed for these planar beams (discussed in further detail in the next chapter) provide insight about the relationship between geometric and material parameters, and the resulting actuation and loading responses. The results have been verified by Moored's numerical model, but experimental validation is necessary in order for these structures to be used in an artificial fin. Houle [113] experimentally tested the loading response of cable-clustered planar tensegrity beams in comparison to numerical simulations, however Houle's work is insufficient in the current context because the optimal cable routing strategy for planar beams was not tested. Therefore experimental validation of the optimal routing strategy is carried out in the present study.

Overall, a review of the background literature draws out several important concepts that directly motivate the work presented in this study. Conventional underwater vehicle technology is lacking in several functions where biological swimmers excel and the swimming style of oscillatory rays has been identified as a desirable model for a biologically-inspired underwater vehicle. The flapping kinematics of these rays is dominated by a large span-wise curvature of the fins, with a superimposed undulatory component. Many studies have been conducted on the propulsive performance of various types of pitching/heaving foils, but investigations that directly relate the specific three-dimensional kinematics of oscillatory rays to swimming performance are lacking. Relevant experimental work suggests that a fin combining both large span-wise curvature and a traveling wave component could perform better than either kinematic component alone. Active tensegrity structures have been identified as a viable method for recreating batoid kinematics, but experimental validation of their mechanics is necessary, and so far active tensegrity has not been physically implemented as a batoid fin. All of these findings lead to the experimental study of active tensegrity structures, their incorporation into a robotic pectoral fin, and the subsequent measurement of swimming performance using such a fin. 


\section{Chapter 3}

\section{Validating Tensegrity Mechanics}

It has been established that batoid rays exhibit coupled span-wise and chord-wise deformations of their pectoral fins that result in what is believed to be highly efficient thrust production. To better understand the relationship between these fin motions and swimming performance, the current study uses a tensegrity-based artificial fin to create kinematics similar to those seen in biology (chapters 4 and 5). Underlying this study is the assumption that replicating the fin kinematics of biological swimmers is key to replicating their performance, and thus to exploring and understanding their swimming capabilities. Any attempt to accurately mimic the kinematics of the biological fins using active tensegrity structures must start with a validated design process that can predict the loaded response as a function of known parameters.

This chapter reviews the mechanics of planar, cable-clustered active tensegrity structures, showing how they can be designed to achieve large span-wise bending and resist external loads. These mechanics solutions are then validated through experimental testing. The validation of external loading resistance for a static tensegrity beam demonstrates the feasibility of implementing these as active structures in an artificial fin. In the next chapter, the design of an artificial fin using active tensegrity is described and validations of active deformation are demonstrated. 


\subsection{Tensegrity Mechanics}

As noted in Chapter 2, active tensegrity structures have been identified as a class of structures with the potential for reproducing the kinematics of batoids. In addition to achieving complex deformations, active tensegrities can be designed to have high stiffness-to-mass ratios and can take advantage of remote actuation schemes, with actuators migrated out of the structure. High stiffness structures are desirable in this study so that fin kinematics are minimally affected by the hydrodynamic forces generated by fluid-structure interaction. Remote actuation schemes both remove mass from the structure (reducing the energy to actuate) [4] and enable the design of slender structures which can fit within the envelope of a ray fin.

While numerical solutions exist for determining the actuation and structural response of general three-dimensional geometries and configurations [39], the process of analysis, form-finding, and critically, construction of these tensegrities is complex, occluding the relationship between design parameters and structural response. For the purposes of this study, simpler tensegrity structures - namely, two-dimensional planar beam $\rrbracket^{1}$ that provide a bending mode - are considered excellent candidates to replicate the span-wise bending deformation of a batoid fin. These simpler structures lend themselves to an analysis that elucidates the relationship between design parameters and structural response.

Moored et al. carried out extensive analysis (analytical and numerical) to design and predict the response of active tensegrity structures [5]. It was proposed that these structures could be used as the foundation for a robotic pectoral fin. For example, a series of active beams could be used to create the necessary tip deflection and traveling wave component similar to that observed in a candidate ray. For a successful design, it is necessary to calculate both the amount of input actuation needed to achieve the required flapping amplitude and the response of this structure due to external loading

\footnotetext{
${ }^{1}$ These beams can be either class 2 or class 3 , depending on whether or not the vertical elements are struts or cables - this modification does not significantly change the mechanics of the active bending mode or response to tip loading.
} 


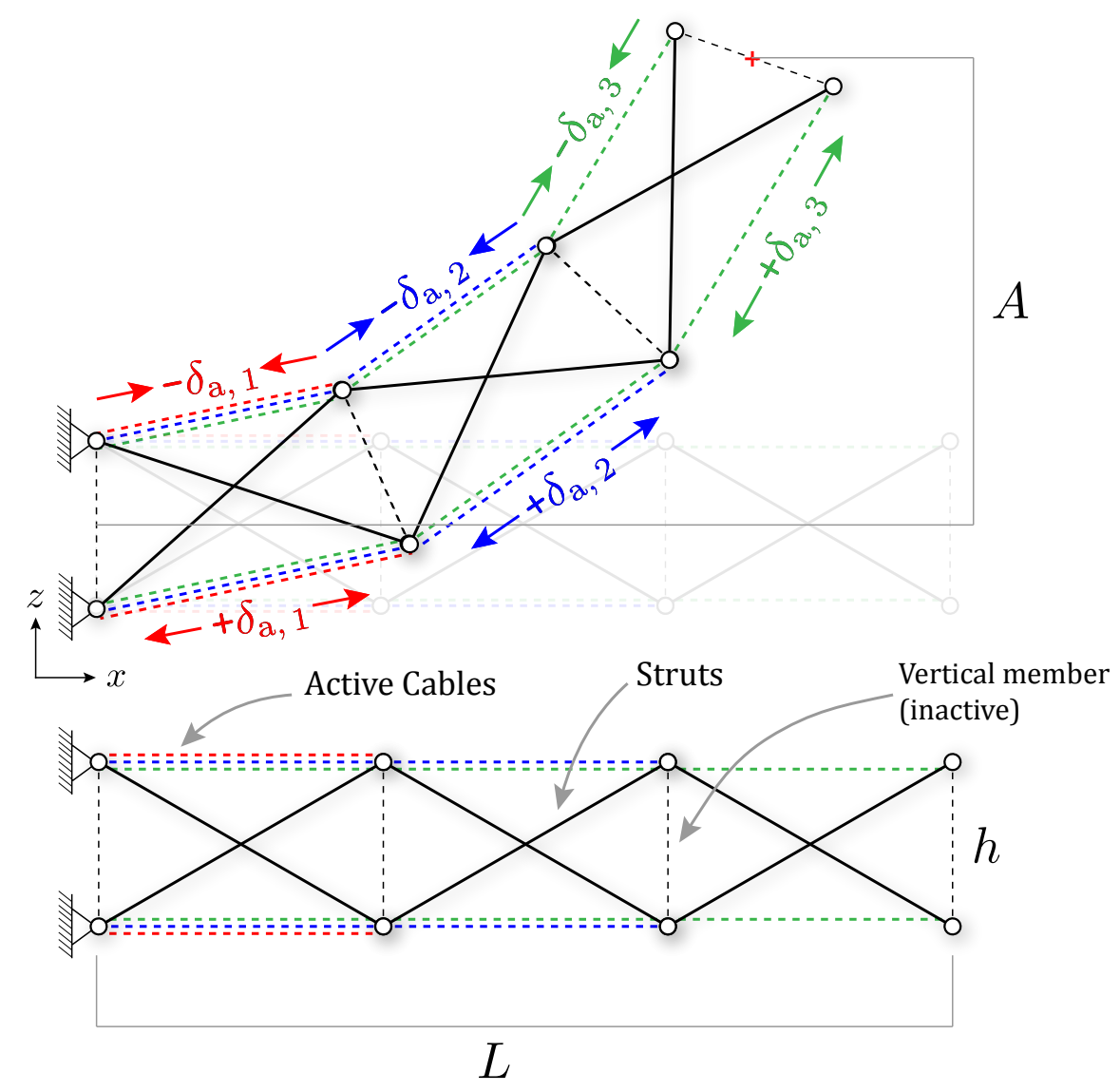

Figure 3.1: A tensegrity beam with total length $L$, made up of $N=3$ planar cross unit cells, showing neutral position and actuation to amplitude $A$. MC routing is also shown schematically, with a single cable (red) corresponding to the first cell, another cable (blue) corresponding to the second cell, and a final cable (green) corresponding to the third cell. In this antagonistic actuation scenario, the amounts of cable contraction (upper) and release (lower) for each cell, $\delta_{\mathrm{a}, j}$, are equal in magnitude but opposite in sign.

(coming primarily from hydrodynamic forces). A review of the relevant mechanics follows.

Moored et al.concentrated on beams comprised of planar tensegrity cross unit cells (figure 3.1) because this unit cell gives the desired bending mode, has been identified as a high stiffness-to-mass topology [114], and conveniently lends itself to a straightforward analysis.2 Using this unit cell, several cable routing schemes are possible, each of which can produce equivalent unloaded actuation but which

\footnotetext{
${ }^{2}$ The analysis relies on a configuration in which each cell of the beam has the same geometry.
} 
have different load bearing characteristics. A routing scheme termed Multiple Cablerouted ${ }^{3}$ (MC) has been identified as the optimal choice in terms of stiffness and therefore will be the only routing scheme discussed in this work. In an MC routing scheme, each cell has its own pair of active cables that are routed along the cables of previous cells, resulting in multiple cables per cell (illustrated in figure 3.1). For this topology and routing, a linearized approximation of the input actuation for the cables of the $j$ th cell $\delta_{\mathrm{a}, j}$, needed to produce a vertical tip amplitude, $A$, is derived for an active tensegrity beam comprised of $N$ planar cross unit cells:

$$
\delta_{\mathrm{a}, j} \approx \frac{A h j}{N L}
$$

$L$ is the total length of the beam and $h$ is the height of the beam. The actuation scheme for this unit cell is based on the assumption that it is purely antagonistic i.e. for the pair of top and bottom cables, $\delta_{\mathrm{a}, j}$ refers to a change in the length of each cable that is equal in magnitude but opposite in sign. Note that for equal actuation of each cell in an MC actuation scheme, the actuation for the $j$ th cell is simply $j$ times as large as for the base cell. Given this simple relationship, we can find the approximate input cable actuation required for a tensegrity beam composed of planar crosses, with any number of cells, and any aspect ratio $L / h$ to give a desired vertical tip amplitude.

Next, the response to an external load, $P$, applied to the beam tip $5^{5}$ is considered. There are two important aspects of the response to consider: (1) the change in force and thus pretension level in each of the cable elements, and (2) the deformation of the beam due to cable element strain. The former is relevant mostly because it determines the limit of validity for the latter. That is, the predictioned deformation response relies on all cables elements having nonzero tension, and if the tension in

\footnotetext{
${ }^{3}$ Multiple cable routing features a pair of active cables for each cell which are routed along the the active cables of the previous cell

${ }^{4}$ Note that $j$ increases from base to tip, such that $j \equiv 1$ for the base cell and $j \equiv N$ for the tip cell.

${ }^{5}$ Applied in the plane of the beam and perpendicular to the length of the beam.
} 


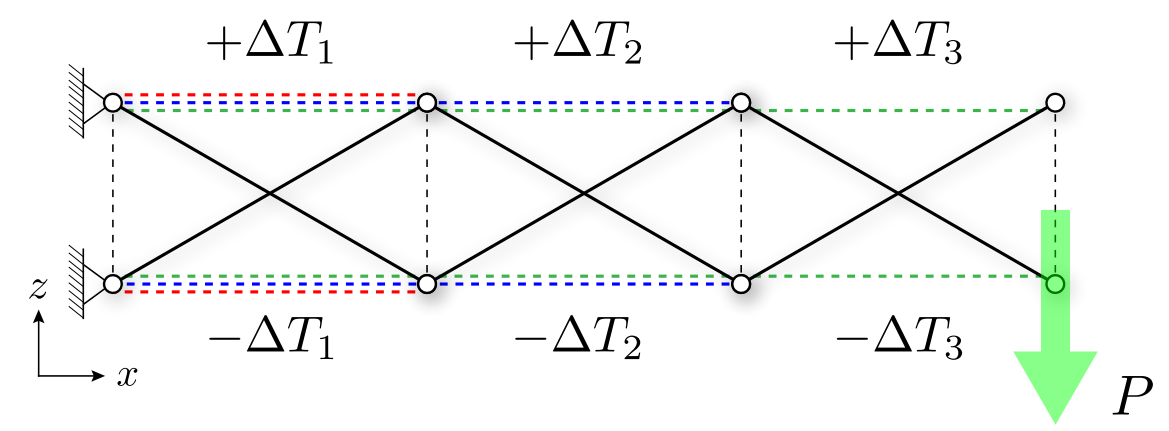

Figure 3.2: Tensegrity beam with external tip load, $P$. This tip load causes a change in tension for the top and bottom cables. Here, a downward $(-z)$ force causes the tension in the top cables to increase, and tension in the bottom cables to decrease. This follows expectations for ordinary beam bending, which places one side of the beam in tension and the other in compression.

any cable element drops to zero because of an external load, then the deformation predictions are no longer valid. The load at which this occurs is termed the critical slackening load.

Moored et al. found the change in tension in the $j$ th cell, $\Delta T_{j}$, associated with an applied tip load, $P$, to be [5] :

$$
\Delta T_{j}=\left\{\begin{array}{l}
\frac{P L}{N h} \quad \text { if } \quad j \neq N \\
\frac{P L}{2 N h} \quad \text { if } j=N \quad \text { (tip cell) }
\end{array}\right.
$$

Note that the response differs for the tip cell because it is the only cell which does not have another cell attached to its terminal end. As a direct result from equation 3.2. the critical slackening load, $P_{\text {cr }}$, is the load at which $\Delta T_{j}=T_{0, j}$ (i.e. when the decrease in tension is equal to the pretension, the cable is effectively slack):

$$
P_{\text {cr }}= \begin{cases}N T_{0, j} \frac{h}{L} & \text { if } \quad j \neq N \\ 2 N T_{0, j} \frac{h}{L} & \text { if } j=N \quad \text { (tip cell) }\end{cases}
$$


It is optimal to have the bottom ${ }^{6}$ cables of every cell to go slack at the same load level. This is made evident by considering the contrary situation: if a single cable goes slack before others, then either the loading response of the overall beam is unnecessarily softened by this one errant cable, or all the other cables are excessively tensioned, which increases actuation resistance7. Equation 3.3 demonstrates that for equal pretensions in every cell, the cables will not all become slack at the same load.

In order to rectify this situation, the optimal pretensioning ratio for an $\mathrm{MC}$ beam should be set such that the pretension in the tip cell is half that of all other cells, as dictated by equations 3.2 and $3.3 . T_{\mathrm{opt}}=P_{\mathrm{cr}} L / 2 \mathrm{Nh}$, so that for the tip cell $T_{0}=T_{\mathrm{opt}}$ and for all other cells $T_{0}=2 T_{\mathrm{opt}}$. Using these results, the pretension distribution in an $\mathrm{MC}$ beam can be properly prescribed to match an assumed maximum level of loading such that all cables go slack at the critical load, (or such that no cables go slack if some factor of safety is desired), without making the pretensions unnecessarily high.

The final important result drawn from this analysis is predicting the amplitude of beam tip deformation, $A_{P}$, due to external tip load, $P$, which is captured in the first order bending stiffness, $K_{\text {bend }}$ :

$$
K_{\mathrm{bend}}=\frac{P}{A_{P}}=\frac{2 N^{2}}{2 N-1}\left(\frac{E_{\mathrm{c}} A_{\mathrm{c}}}{L}+\frac{2 T_{\mathrm{opt}}}{L}\right)\left(\frac{h}{L}\right)^{2}
$$

It can be seen from this result that the bending stiffness scales with number of cells $(N)$, the inverse of aspect ratio $(h / L)$, cable material stiffness $\left(E_{\mathrm{c}}\right)$, cable element size $\left(A_{\mathrm{c}}\right)$, and pretension level $\left(T_{\mathrm{opt}}\right)$. This is a useful result because it allows us to explore how these structural parameters influence stiffness, and we can design

\footnotetext{
${ }^{6}$ Here the convention of a downward $(-z)$ tip load is carried forward, so that tensions in the bottom cables decrease with a tip load applied.

${ }^{7}$ Pretensional actuation resistance is discussed in Moored et al., but is beyond the scope of this section (see section 4.1 below for a discussion the topic). It is sufficient for the objectives of this section to note that pretension above and beyond that required for the critical slackening load increases resistance to actuation. Additionally, increasing pretension levels will increase friction at the joints of the structure, but the contribution of this effect is difficult to estimate and depends upon specific chosen hardware.
} 
accordingly. It should be noted that for high relative stiffness $\left(k_{\mathrm{r}}=E_{\mathrm{c}} A_{\mathrm{c}} / 2 T_{\mathrm{opt}}\right)$, the level of pretension does not contribute greatly to bending stiffness, which is instead dominated by material properties.

In summary, this section has reviewed the relevant design equations provided by Moored et al. that predict the actuation and loading response of planar MC active tensegrity beams as a function of geometry and material properties. The following sections describe how the analytical predictions have been experimentally validated and the results will be used as the basis for designing an active tensegrity beam for use in an artificial pectoral fin.

\subsection{Experimental Validation}

In order to implement a tensegrity beam as the structural basis for an artificial pectoral fin, we must first establish the validity of the design equations introduced in the previous section. Two separate relationships need to be addressed: (1) the input actuation to tip amplitude relationship and (2) the load-displacement relationship. The former will be addressed in section 4.5 using the active tensegrity beam of the artificial fin. The latter is the subject of this section, wherein validation of the loaddisplacement design equations is accomplished through experimentation. A static (non-actuated) MC tensegrity beam is constructed and subjected to external tip loads while measuring tip displacement. The results pave the way for using these beams in an artificial fin, with confidence in their response to external loads.

\subsubsection{Test Setup}

Several features of the idealized tensegrity structures introduced in the previous section make it challenging to create an accurate physical representation: frictionless rotation at all joints, frictionless motion of cables through each node during deformation, absolutely rigid strut members, linear elastic cables, nodes that are essentially 


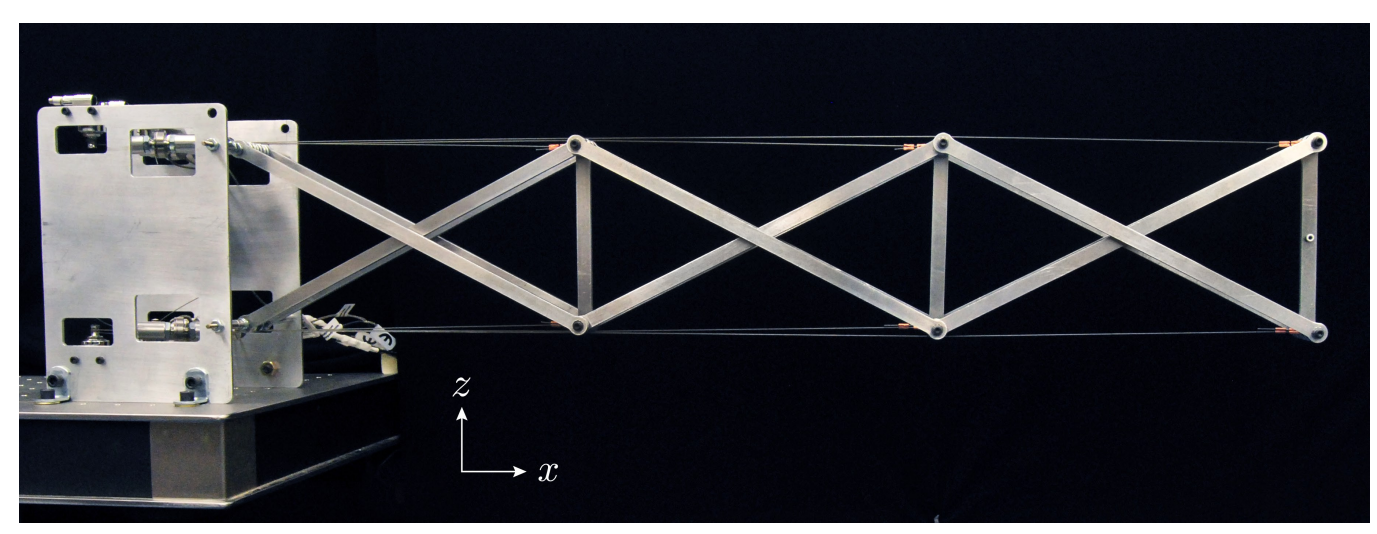

Figure 3.3: Static, 3-cell, MC tensegrity beam used for experiments. Strut elements are machined from aluminum and cable elements are braided stainless steel. On the left side the beam is rigidly attached to the tensioning base. On the right, the tip tracking target is centered on the terminal end of the beam.

points (zero radius), precisely prescribed pretensions in every cable element, and the structure's inherent two-dimensionality. Every effort was made to satisfy these conditions experimentally, and the physical features used to realize them are described in this section. This study uses a class 3, 3-cell beam structure composed of planar tensegrity cross unit cells (see figure 3.3). This topology can be assembled as a class 2 structure, with two struts from adjacent cells meeting at shared nodes, and in this configuration a cable element acts as the vertical member shared between adjacent cells. However, replacing those vertical members with rigid strut elements greatly simplifies construction and does not alter the structural response of interest, which is dictated by the elongation of horizontal cable elements. As for the choice of three cells, using only a single cell structure for these experiments would obviously not demonstrate that the analytical predictions for multiple cell structures hold true, yet a structure with a very large number of cells is cumbersome to construct. Therefore the number of cells was chosen to be three: enough to demonstrate multiple-cell mechanics but few enough to simplify construction.

The rigid strut elements are machined $9.5 \mathrm{~mm}$ wide from $5.0 \mathrm{~mm}$ thick 6061 aluminum plate using a CNC machine with $10 \mu \mathrm{m}$ resolution. The analytical predictions assume the nodes are frictionless, so flanged ball bearings with $0.125 "(3.18 \mathrm{~mm})$ inner 
diameter are inserted in the ends of each strut for low-friction rotation at the joints. The nodes are constructed by joining the strut ends of adjacent cells with steel 5-40 $(0.125 " \varnothing)$ cap screws as axles. In the experimental beam, a pair of parallel struts represent a single compression element and they sandwich the ball bearing pulleys at the nodes. This configuration minimizes twisting and out-of-plane bending of the structure while under load, as it is not possible in this design to have all cables perfectly coplanar. The cable tension elements are made of $0.018 "(0.46 \mathrm{~mm})$ nominal diameter braided stainless steel aircraft cable with a rated breaking strength of 40 lbf $(178 \mathrm{~N})$. The cable elements are laid out in the beam structure according to the $\mathrm{MC}$ routing scheme, routing through some nodes and terminating at others. Cables are routed through a node by wrapping around a ball bearing pulley, to simulate the frictionless routing of the analytical predictions (see figure 3.4). The cables are terminated at nodes in the structure using copper cable crimps around the pulleys. The overall structure has a length $L=0.72 \mathrm{~m}$ and height $h=0.12 \mathrm{~m}$, giving a beam aspect ratio of $L / h=6$.

One assumption of the analytical model is that the strut elements are completely rigid and that structural deformation under external loading is due only to axial strain in the cable elements. To demonstrate the validity of this assumption, we can compare the axial stiffness, defined as $k=A_{\mathrm{c}} E / l$, for the two structural elements. $A_{\mathrm{c}}$ is the cross-sectional area for each element, $E$ is elastic modulus of each element, and $l$ is the length of each element. In addition to the geometry of the elements, it is necessary to determine the elastic modulus for the strut and cable, which is essential for predicting the loading response of the structure (see equation 3.4). For the struts we assume a standard value for 6061 aluminum [115], $E_{\text {strut }}=70 \mathrm{GPa}$. Since the elastic modulus and even the alloy of stainless steel for the braided cables are unknown, the modulus is obtained by tension testing according to standard ASTM A 931-08 [116].

Five cable samples were prepared with the same terminations as used in the tensegrity beam. These samples were each loaded in tension at a crosshead displacement 


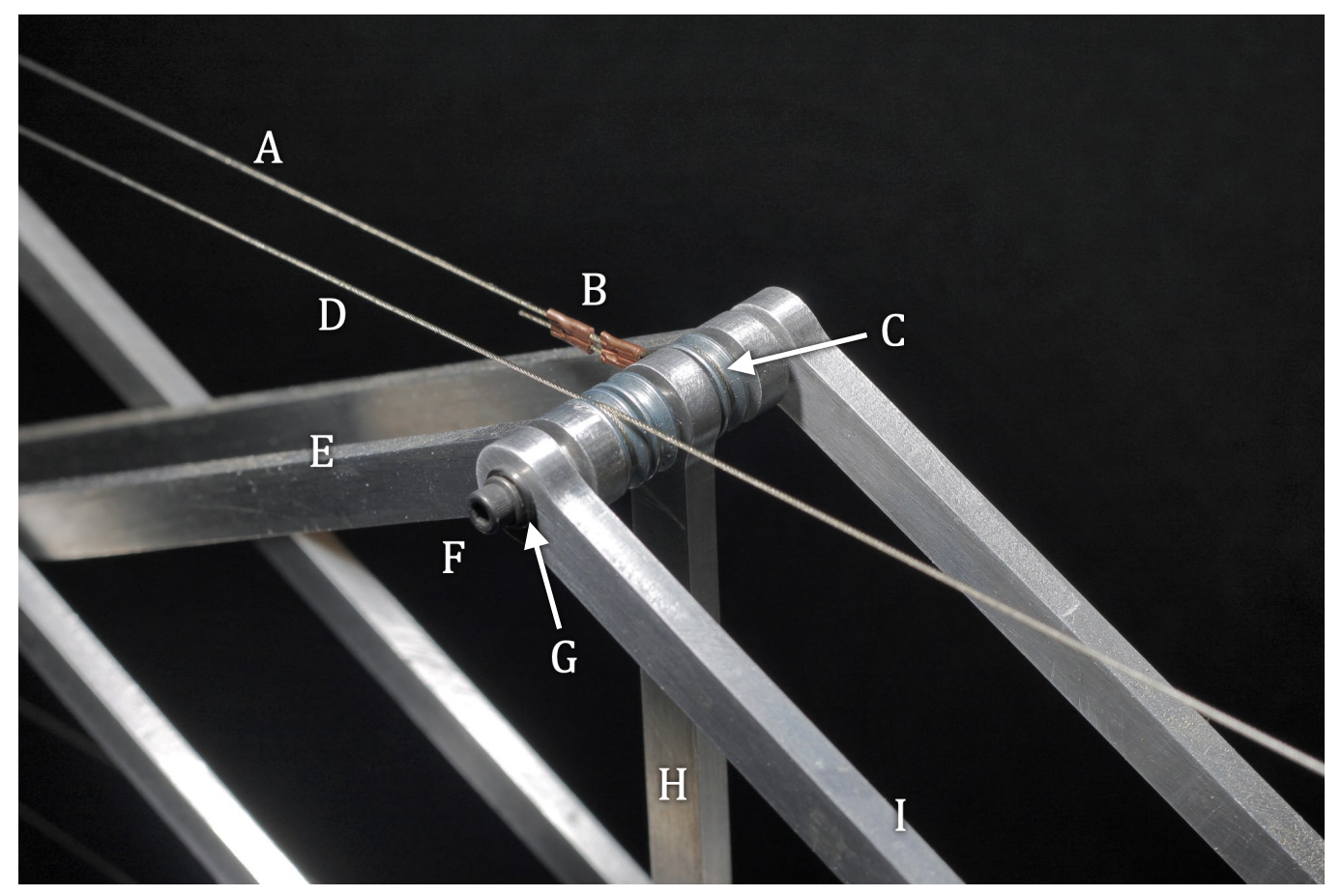

Figure 3.4: Detail of joint construction, showing the upper node on the static tensegrity beam between cells 2 and 3 . The cable for cell 2 (A) terminates with copper crimps (B) around a ball-bearing pulley (C). The cable for cell 3 (D) is routed around a pulley at the node. Struts from cell 2 (E) and cell 3 (I) share this node. The node's axle is made by a cap screw $(F)$, which runs through flanged ball bearings $(G)$ in the strut ends. A vertical strut $(\mathrm{H})$ also connects at this node. 


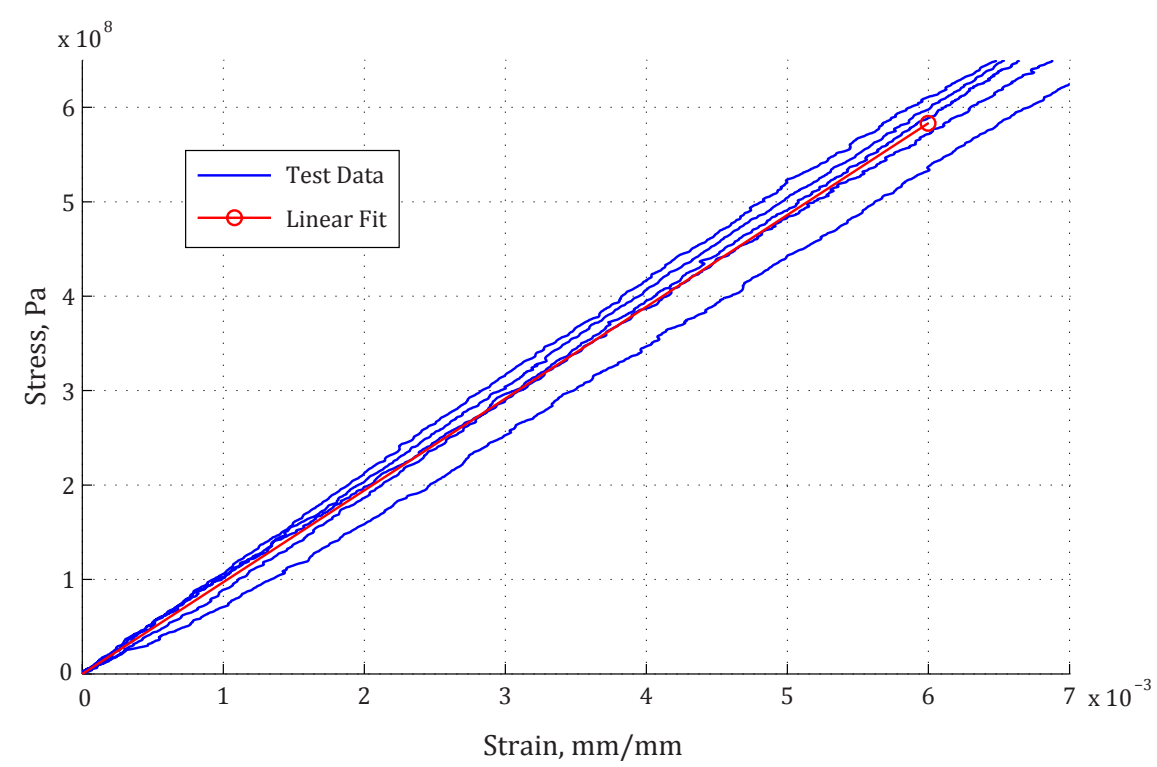

Figure 3.5: Results of tensile testing for 5 samples of braided stainless steel cable used in the experimental MC beam. This linear portion of the stress-strain curves was used to determine the mean elastic modulus, $E_{\text {cable }}$ (plotted in red).

rate of 1 millimeter per minute until failure (complete cable rupture) in an Instron 5848 Microtester. Load readings were taken with a $2 \mathrm{kN}$ load cell and strain values were measured with a laser extensometer, independent from the crosshead displacement. The stress-strain curves produced from this load-displacement data are shown in figure 3.5. The modulus value for each specimen was calculated as the slope of a linear fit to the stress-strain data, up to $110 \mathrm{~N}$ (62\% of the ultimate load in tension tests ${ }_{8}^{8}$. The mean value for the modulus of the cables was found to be $E_{\text {cable }}=97$ GPa, with a standard deviation of $3 \mathrm{GPa}$.

Now to compare the axial stiffnesses we take a worst-case-scenario for the lengths of each, using the value of the longest strut member $\mathrm{I}^{9}$ in the beam $(0.268 \mathrm{~m})$ and the shortest cable member in the beam $(0.24 \mathrm{~m})$. Using the measured modulus of the cable, the calculated stiffness for the shortest cable element is $k_{\text {cable }}=68 \mathrm{kN} / \mathrm{m}$ whereas the stiffness for the longest strut element is $k_{\text {strut }}=25 \times 10^{3} \mathrm{kN} / \mathrm{m}$, making

\footnotetext{
${ }^{8} 110 \mathrm{~N}$ is the maximum tension in any cable during the tensegrity load-displacement experiments.

${ }^{9}$ Note that because of the sandwiched construction of the beam, two struts with the dimensions described previously take the load as one member, thus doubling the effective area.
} 


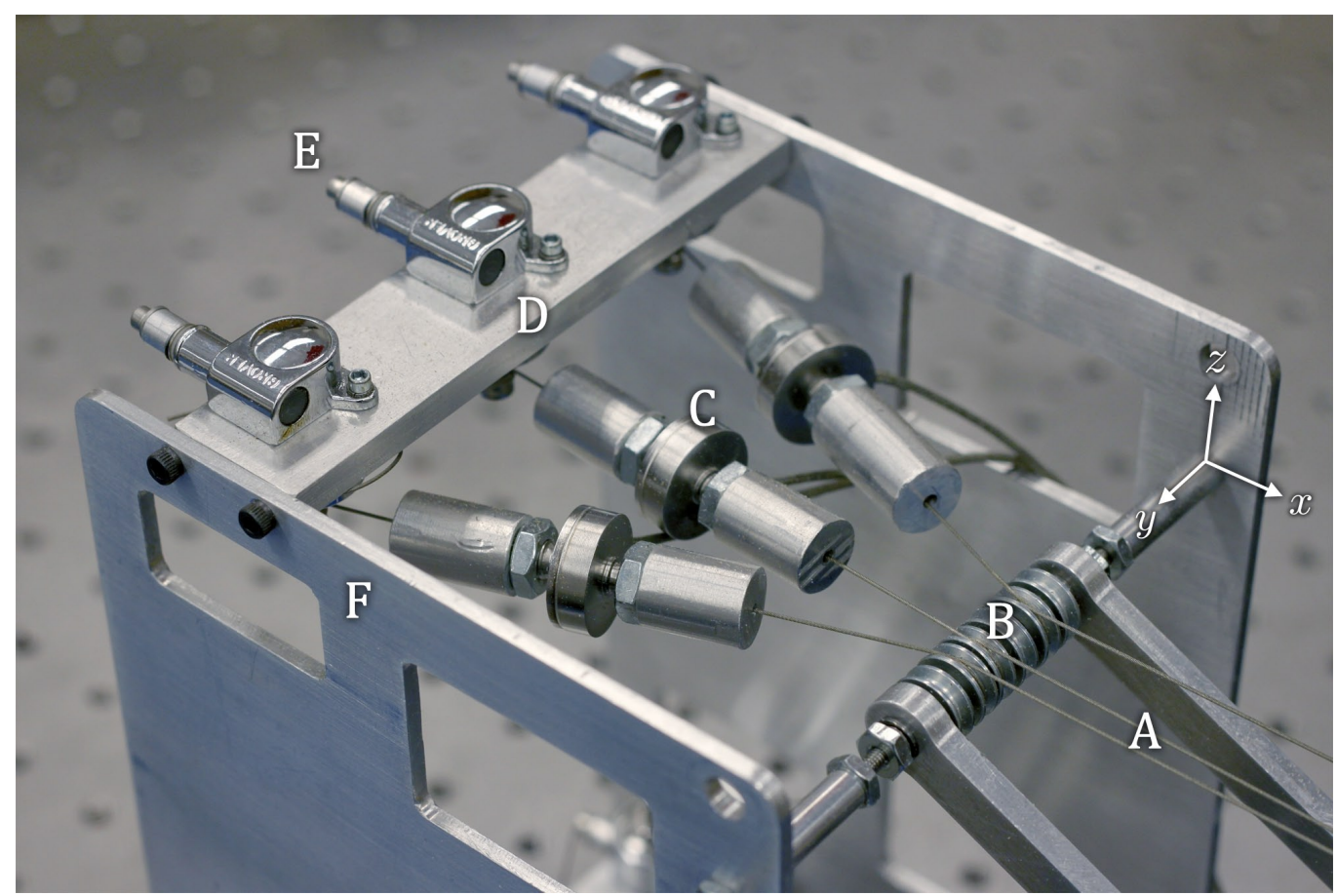

Figure 3.6: Detail of the mounting base for the static tensegrity beam, showing connections for the upper cables. Each cable (A) routes through the upper base node (B) before terminating at an in-line load cell (C). The load cells are then connected to the posts of machine heads (D), which set the pretension in each cable by tuning at $(\mathrm{E})$. All hardware is secured to a rigid base $(\mathrm{F})$, also machined from aluminum.

the struts approximately 370 times as stiff as the cables. Based on this result we can proceed with confidence that our rigid strut assumption is valid.

To measure the tension in each cable, a strain gauge-based load cell (Omega LC201-25) is attached at its terminus and then connected to a tensioning mechanism, as shown in figure 3.6. These in-line load cells are necessary for both proper pretensioning and to validate the predictions for change in cable tension due to external loading. The tensioning mechanism for each cable is based on a guitar machine head, which is a compact, non-backdrivable worm gear set. This hardware allows for fine tuning of the pretension in the structure, which is of critical importance to setting a predetermined slackening load. This base of the structure is fabricated from machined aluminum plates and is rigidly secured to an optical table. 


\subsubsection{Experimental Procedure}

With the tensegrity beam constructed as described above, it is mounted in a vertical orientation, such that self-weight of the beam in bending does not affect the tension in the cables. In this orientation, the structure is pretensioned to the specified optimal pretension ratio for an $\mathrm{MC}$ beam of $T_{0}=[2,2,1] T_{\mathrm{opt}}$, as discussed in section 3.1. Since the purpose of these tests is to show the transition of bending stiffness at the predicted critical slackening load, a range of loads spanning the pre-critical and post-critical regimes was applied. Also, in order to maximize the signal-to-noise ratio of the measurements, the maximum external load is chosen to place maximum tension levels near the full-scale output of the load cells. Since the full-scale output of these load cells is $25 \mathrm{lbf}(111 \mathrm{~N})$, an optimal pretension of $T_{\mathrm{opt}}=19.6 \mathrm{~N}$ and a maximum external tip load of $27.5 \mathrm{~N}$ (administered as $2.8 \mathrm{~kg}$ of brass weights) should give a maximum cable tension 10 of $94 \mathrm{~N}$, or $85 \%$ of the load cell's dynamic range in tension.

The output voltages of all 6 load cells are measured using a National Instruments USB-6259 data acquisition device. The loads are sampled via LabVIEW at a rate of $100 \mathrm{~S} / \mathrm{s}$ and the mean value over 100 samples was recorded for each load. Pretensioning of the cables is accomplished by rotating the wormshaft on each of the tensioning mechanisms while monitoring a live reading of the measured load in the corresponding cable's load cell. The process is recursive, due to the fact that the prestress states of each cell are not independent, so the tensions are adjusted from the base cell outwards repeatedly until the pretension for each cable is within $0.5 \%$ of the target value. The beam is then mounted in a cantilever position (with the plane of joint rotations perpendicular to the plane of the ground) so that weight applied to the beam tip will act in the plane of the beam.

With the structure properly pretensioned, load-displacement tests can be carried out. Loads are applied via hanging masses from the tip of the beam. Cable ten-

\footnotetext{
${ }^{10}$ Using equation 3.2
} 
sions are recorded using the load cells and beam tip displacement is recorded with photographs. Figure 3.7 shows the beam under load in a typical experiment. By measuring the relative displacement of the target between loaded and unloaded positions, the vertical displacement of the beam due to each load is known.

The process of measuring the displacement distances for each photograph was carried out using MATLAB, in order to avoid human error in determining the target positions. Using a photograph of the beam tip in its unloaded position, a region of interest (ROI) containing the target is defined in order to set the origin, from which all distances can be calculated. The code then searches all subsequent tip-loaded images for pixels similar to those found in the ROI, using an image cross-correlation technique. The result is a set of $[x, z]$ pixel coordinates which correspond to the set of applied loads and are relative to the unloaded neutral position. An illustration of the displacement measurement technique is shown in figure 3.8 . The vertical $(z)$ component of each displacement is then transformed into real units by measuring the pixel length of an element in the photograph of known length (specifically, the height of the terminal vertical strut) and scaling by this proportionality.

This procedure was carried out for 14 different loads, ranging from $0.2 \mathrm{~kg}$ to 2.8 $\mathrm{kg}$. The lowest load corresponds to $10 \%$ of $T_{\mathrm{opt}}$ and the highest load corresponds to $140 \%$ of $T_{\text {opt }}$, i.e. a range that spans the pre-critical and post-critical regimes. A set of tests was conducted for all of the loads, and then this process was repeated for a total of 5 sets, from which mean values and standard deviations are derived.

\subsubsection{Results and Discussion}

In conducting the load-displacement experiments, it was found that large tip loads caused noticeable bending of the axle comprising the lower base node of the structure, due to the axial forces in the attached struts and the relatively large span of this axle (see figure 3.9). This was the only noticeable deformation of any component which was assumed to be rigid. The movement of this bottom base node is equivalent to 


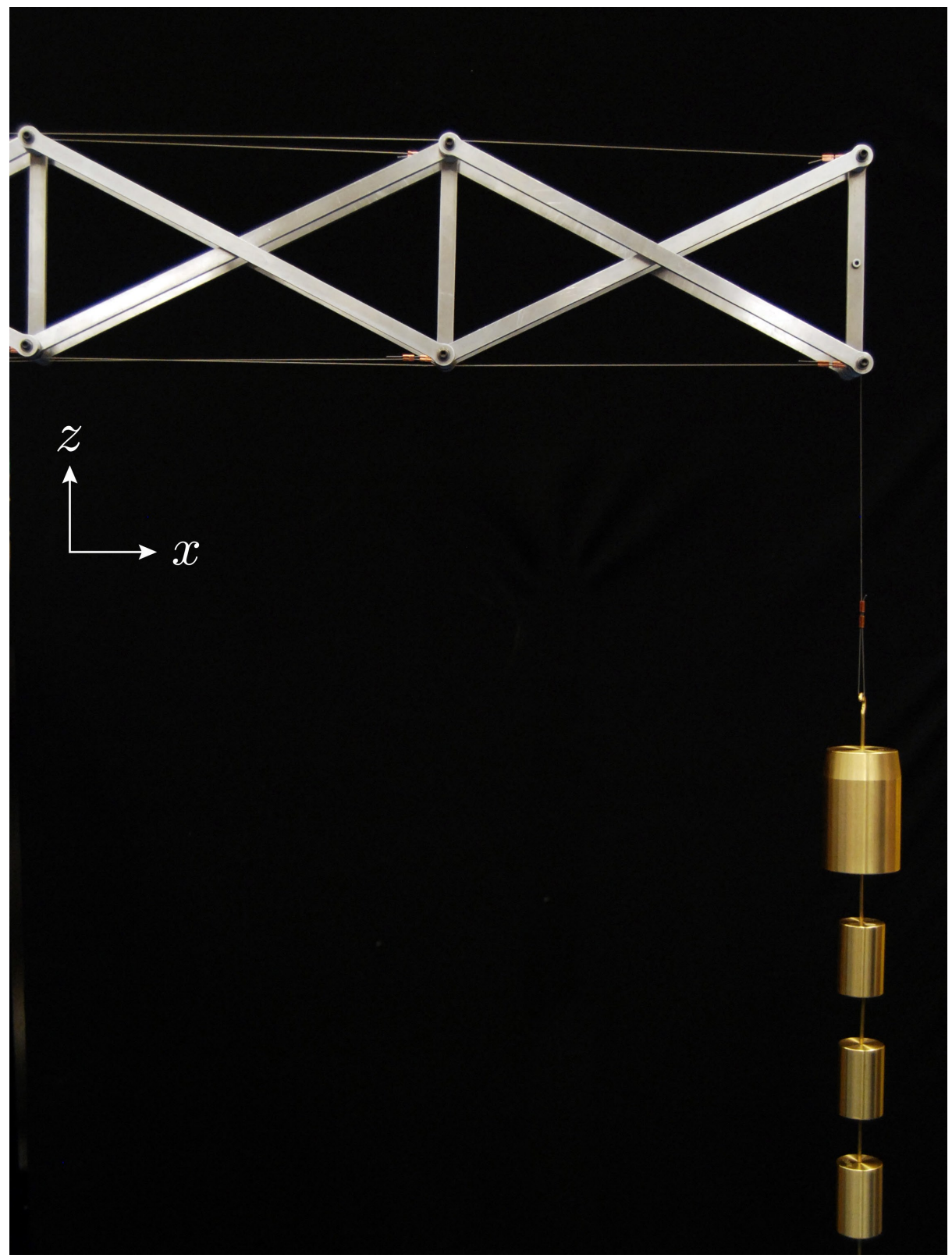

Figure 3.7: During a typical load-displacement test the tensegrity beam is loaded at the tip with brass weights. 


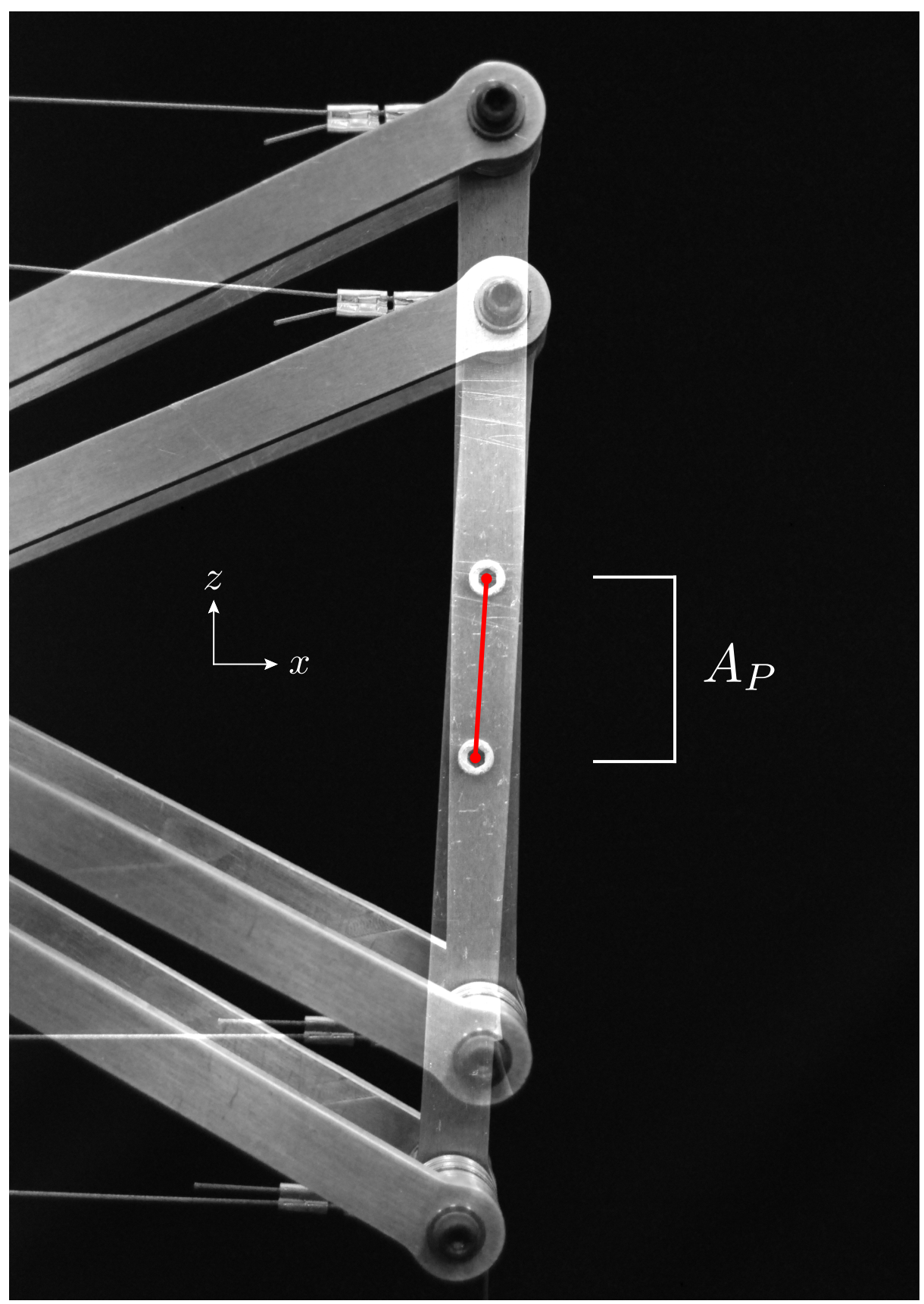

Figure 3.8: Two images from the tip tracking are superimposed to show the amplitude of deformation $A_{P}$ due to tip load $P$. The total displacement (red line) is measured by MATLAB and the vertical $(z)$ component is taken as $A_{P}$. 


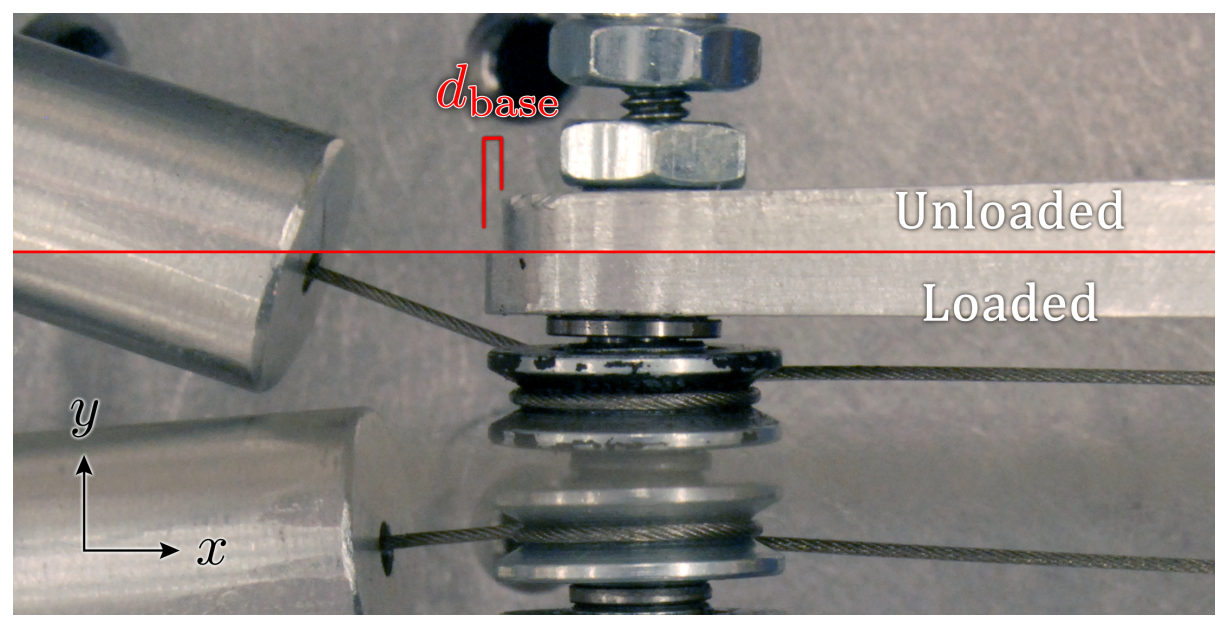

Figure 3.9: Two images overlaid to show the base node axle deforming under load from the attached struts. Here, $d_{\text {base }}=0.64 \mathrm{~mm}$.

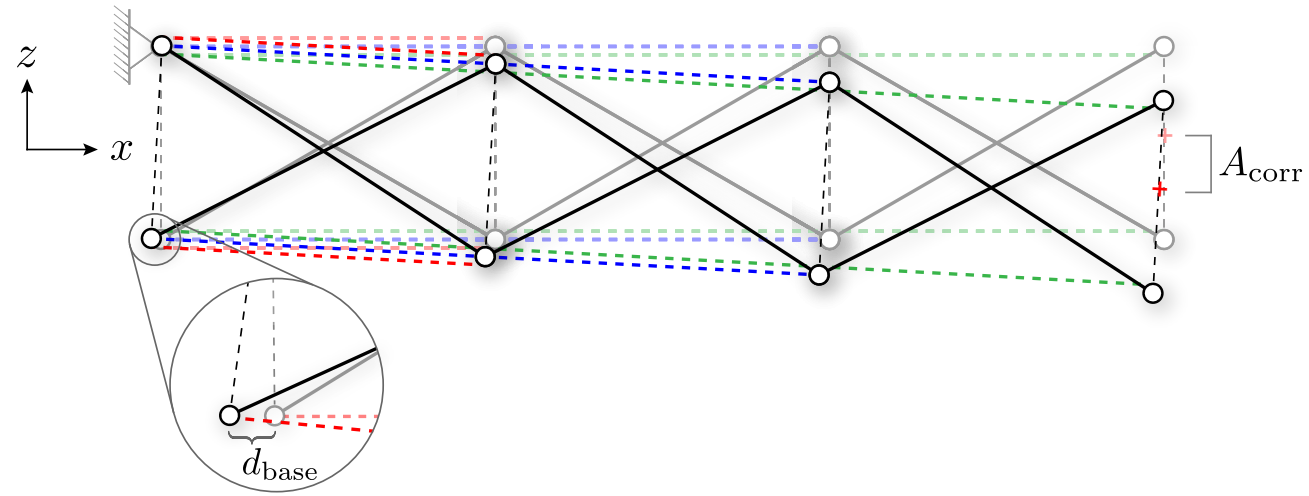

Figure 3.10: Rigid-body rotation of the beam about the upper base node due to movement of the lower base node, $d_{\text {base }}$, in the $-x$ direction.

a rigid body rotation of the beam about the upper base node, as shown in figure 3.10. A correction for this effect is made by measuring the displacement of the base node axle while tip loads are applied. This displacement was measured optically, as in figure 3.9, and the correction for tip displacement is approximately:

$$
A_{\text {corr }}=d_{\text {base }} \frac{L}{h}
$$

Figure 3.11 shows the optically measured lateral base node movement plotted against applied tip load. The load-displacement relationship was highly linear over the range tested. These base node movements can be converted to equivalent tip 


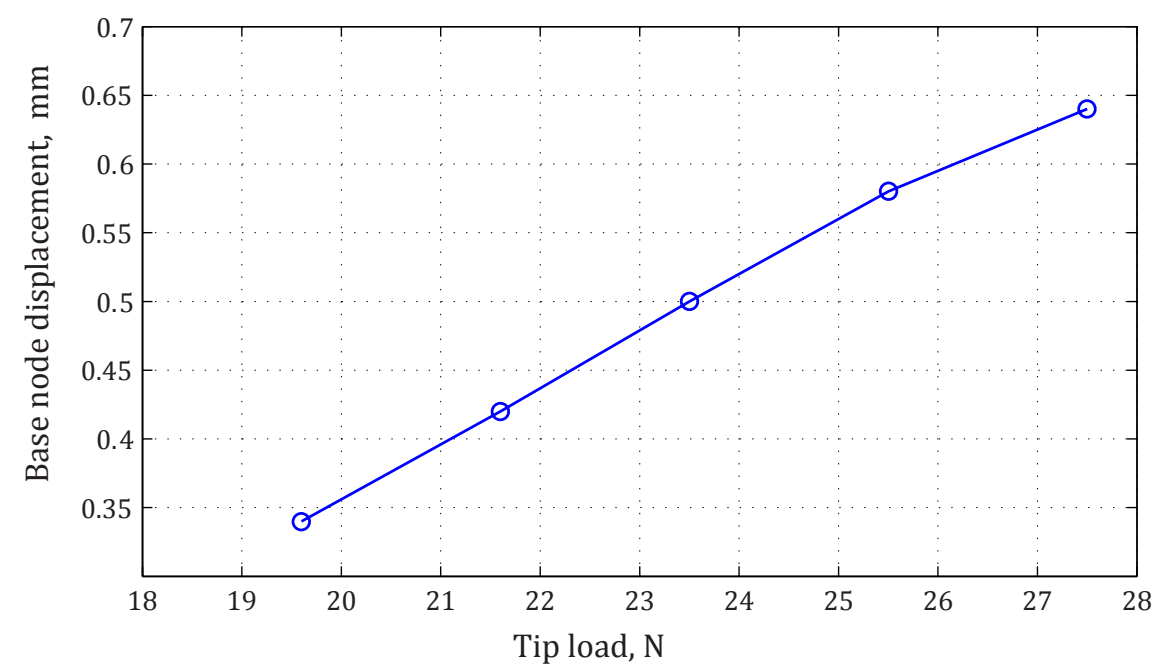

Figure 3.11: Displacement of base node based on optical tracking plotted against applied tip load.

displacements using equation 3.5. The maximum tip displacement due to the deformation of the base node axle, as a percentage of the total tip displacement, was found to be $A_{\text {corr }} / A_{P}=20 \%$. The final load-displacement data for the beam is calculated using both the measured displacements at the tip and the tip displacements due to base node movement, so that $A_{P}^{*}=A_{P}-A_{\text {corr }}$.

During the experiments, it was also observed that the structure had a small but noticeable amount of out-of-plane deformation under load, as shown in figure 3.12 . The source of this deformation appears to be eccentric loading on the nodes of the structure, arising from its inherent three-dimesionality. Both the analytical and numerical models of the tensegrity beam are for an idealized two-dimensional structure, yet for experimental purposes the beam is three-dimensional. As a result the cables are not all coplanar, so they exert loads at termination points within the structure which are eccentric with respect to the idealized two-dimensional plane of the structure. The rotational joints of the structure at each node are made with ball bearings, which have a finite amount of both play and stiffness with respect to radially-induced moments. These physical characteristics of the bearings allow for the struts to rotate out-of-plane relative to one another, resulting in the observed out-of-plane deforma- 


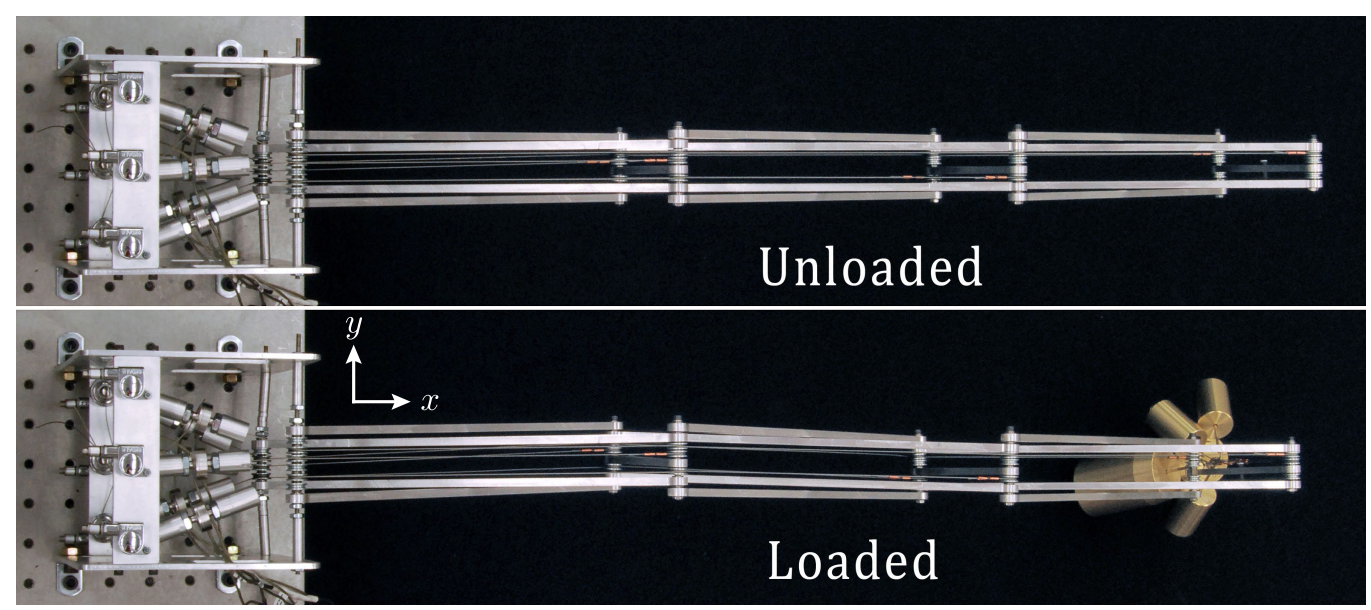

Figure 3.12: Overhead view of tensegrity beam deforming under load, showing outof-plane motion particularly at the nodes between cells 1 and 2. This out-of-plane deformation keeps the bottom cables from becoming completely slack at the predicted critical slackening load.

tions for the overall structure.

The major consequence of these out-of-plane deformations is a delay in slackening: a situation where not all bottom cables become slack at the same critical load. This effect is observed in both load-displacement and change in tension results, which are discussed below. Out-of-plane deformations due to load eccentricities could be further minimized by using stiffer rotational joints with less play, by rigidly connecting the pairs of struts with common nodes, and by doubling the number of cables to create pairs, such that all eccentric cable loads are balanced.

The optically-measured tensegrity beam tip displacements were averaged over 5 sets of tests, and the standard deviations (normalized by the mean displacement values at each load) were calculated, as shown in figure 3.13 . The maximum deviation is about $8 \%$, which was for the lowest load. That deviation is particularly high percentage-wise because the displacement at that load was rather small, at only 0.89 mm. All other deviations were no larger than $1 \%$ of the mean value.

Figure 3.14 presents the experimental results for tip displacement of the tensegrity beam (corrected for base node movement as noted above) plotted against applied tip load. Also shown are plots for the analytical solution (given by equation 3.4) and 


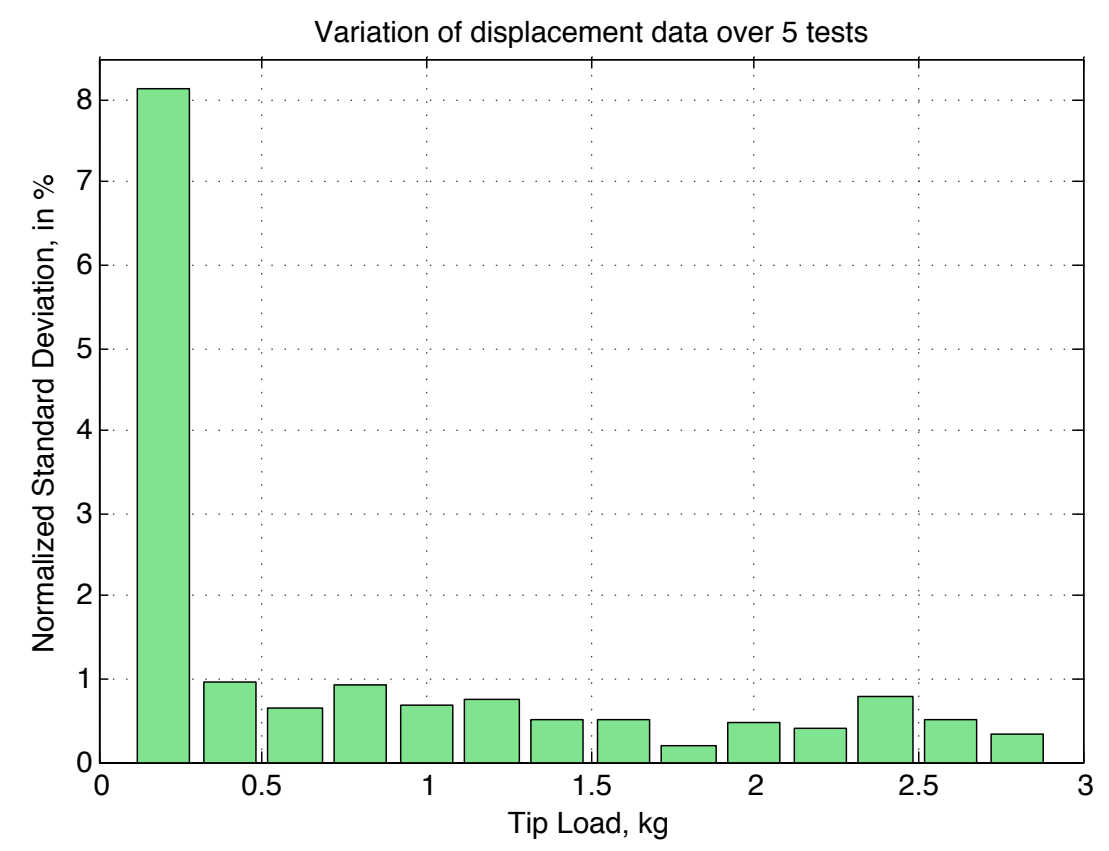

Figure 3.13: Standard deviations of optically measured tip displacement data, normalized by the average displacement value at each load level.

for the full numerical solution (as presented in [5]). Overall, the experimental results demonstrate excellent agreement to the analytical prediction for loads less than the critical slackening load. The analytically predicted first-order bending stiffness is $K_{\text {bend, anly. }}=2217 \mathrm{~N} / \mathrm{m}$ and the slope of the pre-critical experimental data yields a stiffness of $K_{\text {bend, exp. }}=2162 \mathrm{~N} / \mathrm{m}$, a difference of less than $3 \%$.

Approaching the critical slackening load, the stiffness of the structure begins to drop. Ideally, the drop in stiffness should occur right at a normalized tip load of $P / T_{\text {opt }}=1$, however we see from the experimental results that this drop instead occurs near $P / T_{\mathrm{opt}} \approx 0.9$. The reason for this discrepancy is the self-weight of the structure, which acts as an additional load and thus critical slackening begins at a lower normalized external tip load. Self-weight is included in the numerical model, and accordingly the numerical results show a transition from pre-critical to post-critical stiffness occurring around the same external load as in the experimental results.

As expected, the analytical prediction for stiffness is not valid in the post-critical 


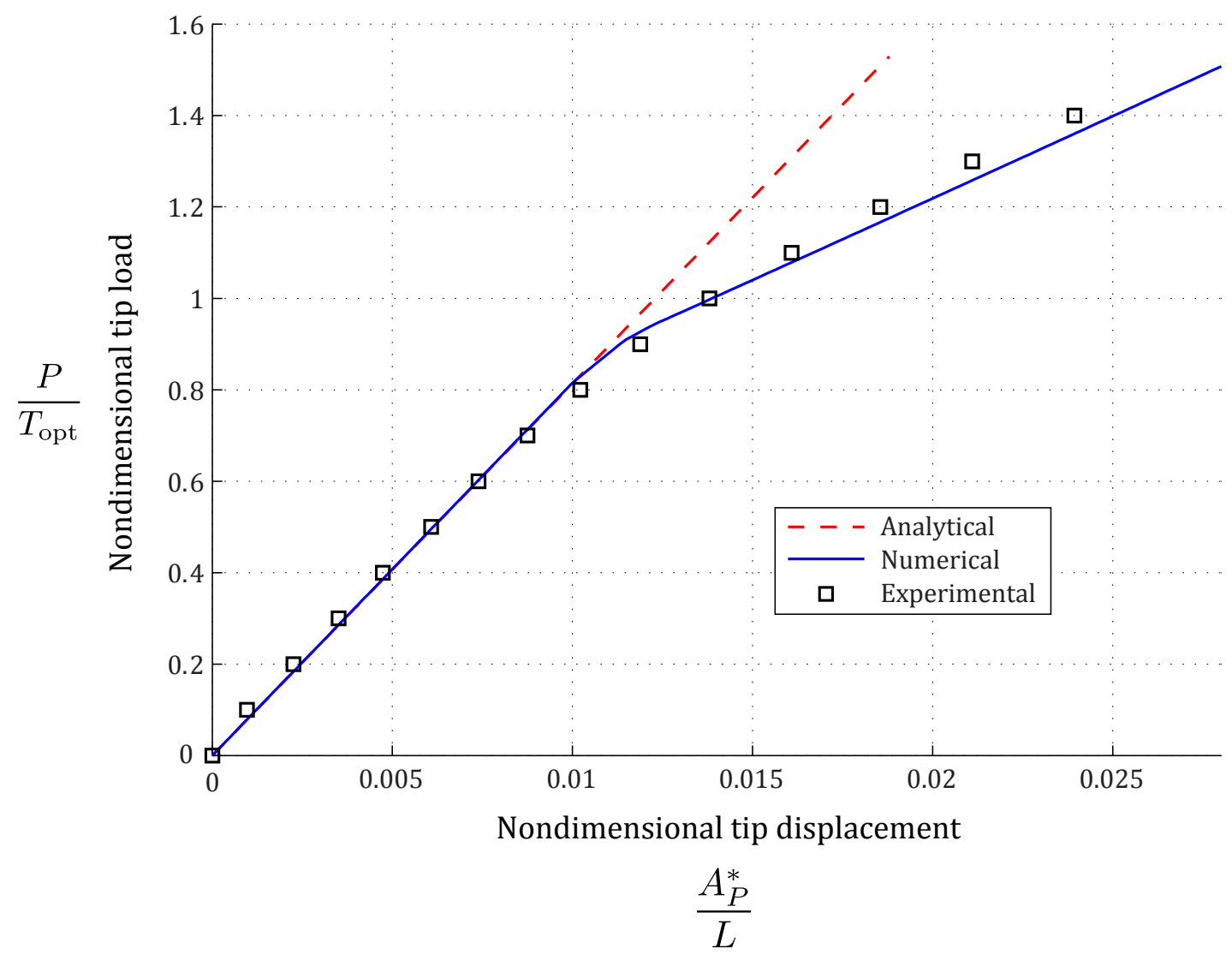

Figure 3.14: Load-displacement data plotted nondimensionally, with the tip load normalized by the pretension $T_{\mathrm{opt}}$ and the tip displacement reported as fraction of beam length. Experiments closely match the analytically predicted bending stiffness in the pre-critical regime. In the post-critical regime, the experiments follow numerical predictions, with slight deviations due to three-dimensional effects on the beam. Standard deviations for the experimental displacements are small enough that they fit within the markers used, so they are not shown. 
regime, where the experimentally measured stiffness settles to a lower value that is $44.4 \%$ of the pre-critical value. The numerical simulation predicts a post-critical stiffness that is $44.5 \%$ of the pre-critical value. So again the experimental results in the post-critical regime are in good agreement with the numerical simulation, which does account for critical slackening (and the associated redistribution of loads).

There is some discrepancy in these post-critical results however, with the experimental data showing slightly smaller displacements than expected at loads above $P / T_{\text {opt }}=1$. This is due to the delay in slackening discussed above: while the postcritical stiffness does settle out very close to the predicted value, it does so over a larger range of loads than is expected because of the delay in slackening - there is not a sharp transition from pre-critical to post-critical stiffness as is seen in the numerical simulation. As a result, the experimental post-critical displacements are offset, shifted left (in figure 3.14 from this delay in slackening. The delay in slackening from out-of-plane deformations is even more evident in the experimental results for cable tension.

Figure 3.15 shows the tension in each cable, reported as force density values, over the range of tip loads. Force density, defined as the force in each element divided by its length $(\lambda=T / l)$, is used because of its prevalence in tensegrity mechanics [4]. Here the force density in each element is normalized by the force density in the tip cell of the unloaded structure, $\lambda_{0}=T_{\mathrm{opt}} / L$. Analytical (given by equation 3.2 and numerical results are also shown.

As expected, the bottom cables demonstrate decreasing force densities as tip loads increase, while the top cables demonstrate increasing force densities as tip loads increase. The force densities have different values at zero tip load for several reasons: the optimal pretension distribution dictates that not all cells have the same pretension, the effect of self-weight changes these initial cable pretensions, and the force densities are scaled (inversely) by the length of each cable, which increases from base to tip. There is generally good agreement between experimental results and the an- 


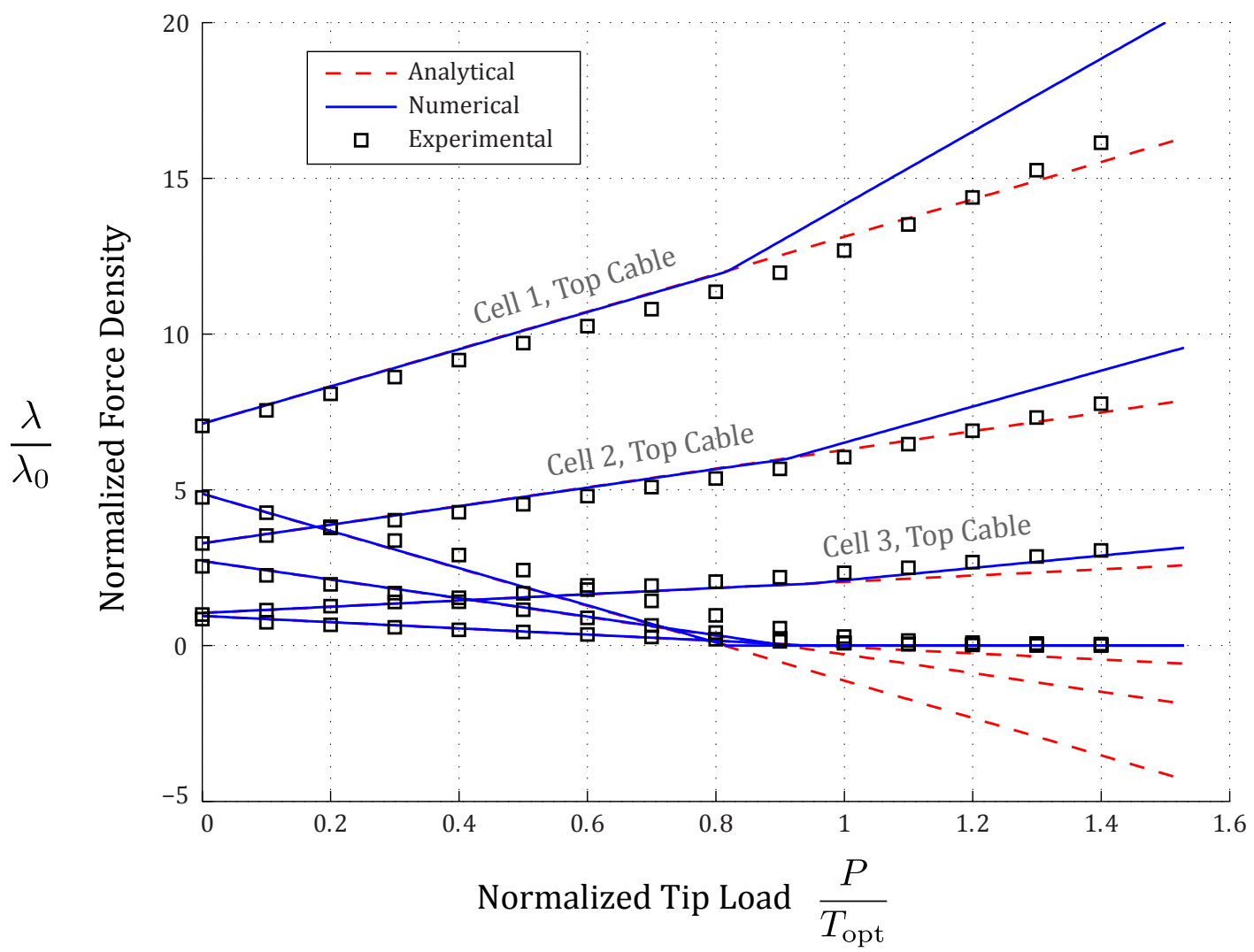

Figure 3.15: Tension in each cable, reported as normalized force density. In the top cables, tension increases with tip load, while in the bottom cables, tension decreases with increasing tip load. Most notably, this plot shows delay in critical slackening from three-dimensional effects, with the result being lower than expected tensions in the top cables for the post-critical regime. 
alytical and numerical predictions. However, there are more noticeable differences compared to the load-displacement results.

The force density in the top cables of cells 1 and 2 are slightly lower than predicted in the pre-critical regime, meanwhile the force density in the bottom cable of cell 1 is noticeably higher than both the analytical and numerical predictions in the range of about $0.3<P / T_{\text {opt }}<1$. The force density in this cable should drop to zero at $P / T_{\mathrm{opt}}=1$, or with self weight included, more like $P / T_{\mathrm{opt}} \approx 0.9$. However it appears that the out-of-plane deformations of the beam lead to a residual tension in this cable, since it is resisting the out-of-plane deformations. During the experiments, it was observed that manually loading the structure in the out-of-plane direction at the nodes in order to straighten the beam resulted in the bottom base cable becoming slack for $P / T_{\text {opt }} \geq 0.9$. It was therefore concluded that the out-of-plane effects discussed previously are the major cause for discrepancies between experiment and the predictions for force density and cable slackening. Even with this delay in slackening, we see a discontinuous increase in cable tension in the top cables when the bottom cables do slacken, which matches expectations.

In summary, the experimental observations validate the analytical predictions for beam stiffness based on geometric design parameters, material properties, and pretension levels. This result directly enables the design of similar beams for use in a fin, with confidence that their resistance to external loading will match expectations. Also, the results clearly demonstrate the effect of critical slackening on beam stiffness, which reinforces the significance of proper pretensioning in these structures. The magnitude of any discrepancies is small enough that we can confidently design MC routed tensegrity beams based on the analytical predictions of section 3.1 . 


\section{Chapter 4}

\section{Robotic Fin Design}

Experimental validation of previously derived tensegrity mechanics allows us to proceed with implementing these structures towards a robotic underwater propulsor. The analysis and design procedure to actuate the active bending mode of a tensegrity beam with minimal resistance is presented. Following this is a walkthrough of the overall design for the robotic fin, considering constraints and desired outcomes. A fluid loading model is used in order to estimate forces on the fin surface, to aid in designing the beam and actuation system. A cam-actuated active tensegrity beam is then implemented into a batoid ray-like pectoral fin. The analytical prediction of the active bending amplitude is compared to experimental measurements in order to achieve the necessary amplitudes for the robotic fin based on prescribed input actuations. The result is a robotic fin with both shape and kinematics that approximate an oscillatory batoid ray, which is used quantify hydrodynamic performance.

\subsection{Modified Antagonistic Actuation}

In order to actuate an active tensegrity beam, some mechanism must be in place to synchronously contract and release cables in accordance with the analytical results

presented in 3.1. Of all possible solutions, it has been shown in [5] that a rotary 

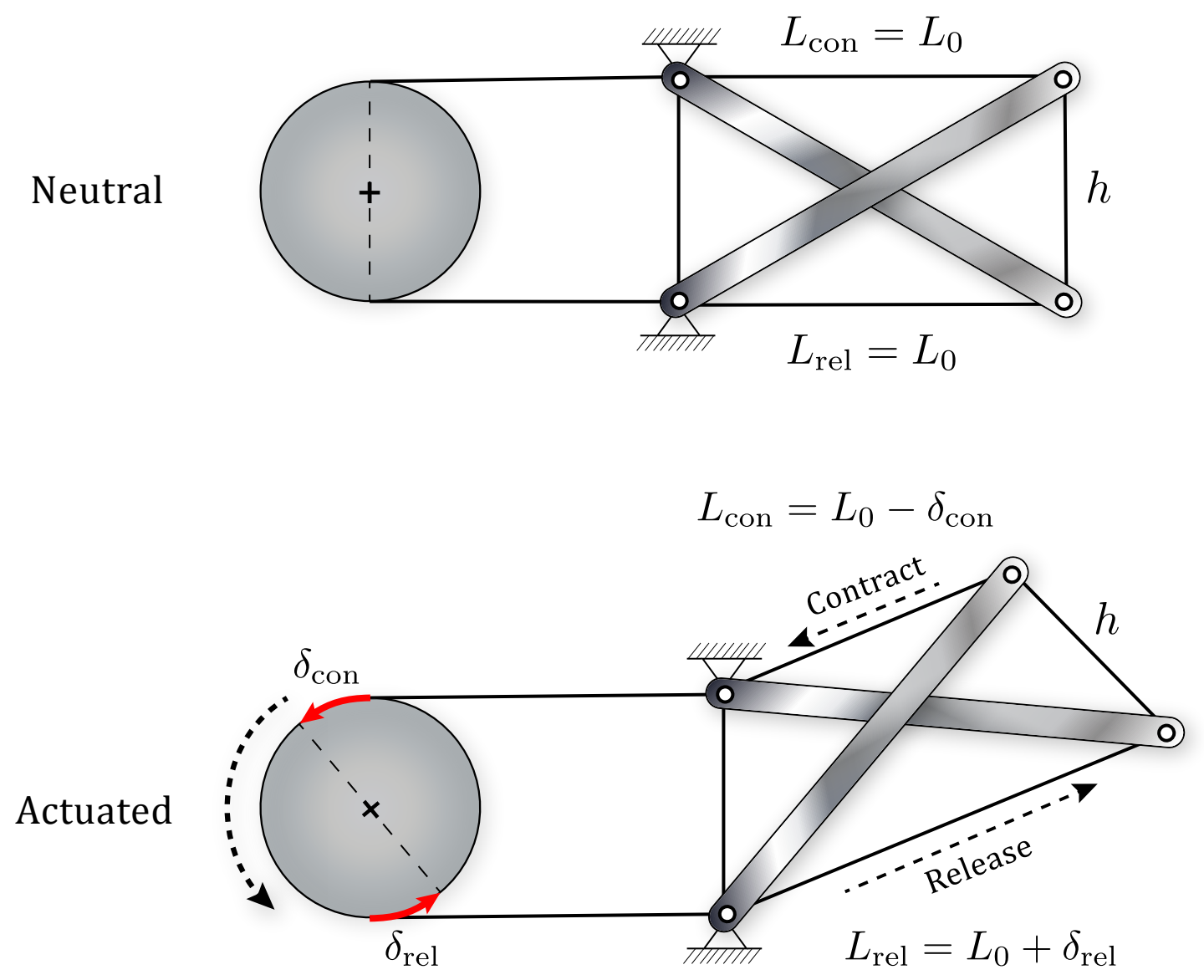

Figure 4.1: A single tensegrity planar cross unit cell, actuated with a rotary drum and demonstrating deformation due to contraction of upper cable and release of the lower cable. In a perfectly antagonistic scenario (circular drum), the cables both change length by the same amount, so that $\delta_{\text {rel }}=\delta_{\text {con }}$. However in a modified antagonistic scenario, the changes in cable lengths differ, so that $\delta_{\text {rel }} \neq \delta_{\text {con }}$.

scheme in which antagonistic pairs are attached to opposite sides of an actuation drum is advantageous. The main advantage gained in this setup is that equivalent pretension forces in each cell's cable pair cancel each other out as equal and opposite torques on the drum, such that no extra effort is required to bear the pretensional loads. In this setup, the proposed shape of the rotary actuation drum is circular, which gives perfectly antagonistic actuation to cable pairs, as shown in figure 4.1 . This antagonistic actuation works well for small angle assumptions upon which the analytical results were derived. 
However, to match the kinematics of batoids, large amplitude beam bending is required and in this regime the small angle assumptions of antagonistic actuation break down. It is straightforward to show that for large deformations of a tensegrity unit cell, the length changes for the pair of cables are not equal. The difference between the length changes represents an induced strain for the antagonist pair of cables - i.e. for perfectly antagonistic actuation, the cell can only deform if one of the cables strains to make up the difference. Thus the prescribed length changes of the cables should match the geometric deformations of the unit cell in order to avoid large loads associated with induced cable strain.

This scenario poses several questions: what is the expected magnitude of forces from induced cable strain as a function of actuation amplitude? What amount of modified antagonistic actuation is needed to avoid this cable strain? And ultimately, how should an actuation mechanism be designed in order to minimize induced cable strain while achieving large bending amplitudes?

To answer these questions and open the possibility of designing a mechanism to produce modified antagonistic actuation, free from undesirable induced cable strain, we should first examine the geometry of a single unit cell (as shown in figure 4.2) to determine the relationship between the lengths of the cell's active cable pair. Quantifying the difference in their length changes as the cell deforms directly indicates the amount of induced cable strain from antagonistic actuation. From the geometry of the tensegrity unit cell, we can use the law of cosines on internal angles to solve for the length of the contraction cable, $L_{\text {con }}$, as a function of the length of the release cable, $L_{\text {rel}}$ :

$$
L_{\text {con }}=\sqrt{2 h^{2}+L_{0}^{2}-2 h \sqrt{h^{2}+L_{0}^{2}} \cos \left[\arccos \left(\frac{L_{\text {rel }}^{2}-L_{0}^{2}}{2 h L_{\mathrm{rel}}}\right)-\arccos \left(\frac{L_{\mathrm{rel}}^{2}+L_{0}^{2}}{2 L_{\mathrm{rel}} \sqrt{h^{2}+L_{0}^{2}}}\right)\right]}
$$




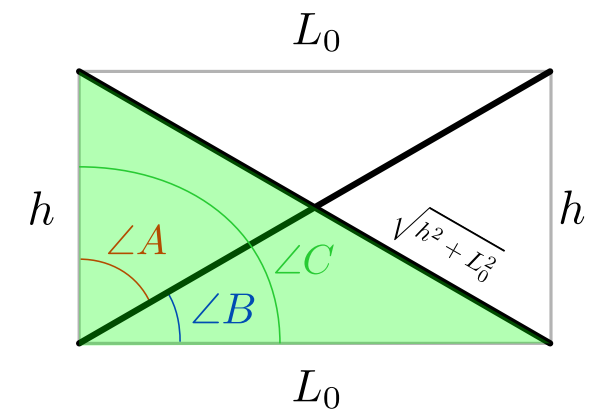

Neutral

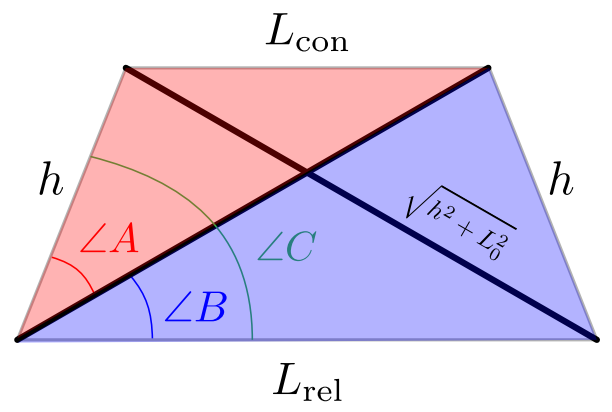

Actuated

Figure 4.2: Internal angles of the unit cell can be used to find a relationship between $L_{\text {con }}$ and $L_{\text {rel }}$. Here, we know $\angle A+\angle B=\angle C$, and the law of cosines is used to describe these angles in terms of both known and desired lengths. Shading is used to indicate the internal triangles used for the derivation of equation 4.1 .

Using the assumption ${ }^{1}\left\{h, L_{0}, L_{\text {rel }}\right\}>0$ and trigonometric identities, the relationship between the two lengths conveniently simplifies to a straightforward equation:

$$
L_{\mathrm{con}}=\frac{L_{0}^{2}}{L_{\mathrm{rel}}}
$$

It is interesting to note that this result is independent of the cell's height $h$ and thus aspect ratio, $A R_{\text {cell }}=L_{0} / h$. Equation 4.2 also demonstrates that the product of the two cable lengths is always a constant $\left(L_{0}^{2}\right)$. Clearly the two lengths are algebraically interchangeable, so this inverse relationship holds for either length as a function of the other. The definition of each cable as contraction or release is arbitrary, since the length changes are symmetric about the neutral, undeformed configuration. For convenience, the remaining relations will be relative to an input actuation prescribed to $L_{\text {con }}$. We can define the lengths of each cable relative to their original lengths as follows:

$$
\begin{gathered}
L_{\text {con }}=L_{0}-\delta_{\text {con }} \\
L_{\text {rel }}=L_{0}+\delta_{\text {rel }}
\end{gathered}
$$

\footnotetext{
${ }^{1}$ For all physical structures, we know that $h, L_{0}$, and $L_{\text {rel }}$ will be positive and real.
} 
For consistency, it should be assumed that $\left\{\delta_{\text {con }}, \delta_{\text {rel }}\right\}>0$ so that $L_{\text {con }}<L_{0}<L_{\text {rel }}$. Using this convention, the length changes in physical units will have positive values. The length changes can be expressed in a number of ways, but the most useful is simply as nondimensional strain values:

$$
\begin{gathered}
\bar{\delta}_{\text {con }}=\frac{L_{0}-L_{\text {con }}}{L_{0}}=\frac{\delta_{\text {con }}}{L_{0}} \\
\bar{\delta}_{\text {rel }}=\frac{L_{\text {rel }}-L_{0}}{L_{0}}=\frac{\delta_{\text {rel }}}{L_{0}}
\end{gathered}
$$

It is important to note that these strains have been defined as positive for convenience, even though $\bar{\delta}_{\text {con }}$ represents "compression" by cable contraction (which is ordinarily a negative strain) and $\bar{\delta}_{\text {rel }}$ represents elongation by cable release.

Up to this point the derivation has been for a single cell. However the results apply to MC beams with $N$ cells using appropriate normalizations. For instance, in an $\mathrm{MC}$ beam the actuation for the $j$ th cell is simply $\delta_{\text {con, } j}=j \delta_{\text {con }}$ and the length of the cable for the $j$ th cell is $L_{j}=j L_{0}$. So if the actuation is normalized as $\bar{\delta}_{\text {con, } j}=$ $\delta_{\text {con }, j} / L_{j}$, then $\bar{\delta}_{\text {con }, j}=j \delta_{\text {con }} / j L_{0}=\delta_{\text {con }} / L_{0}=\bar{\delta}_{\text {con }}$. Hence, the non-dimensional strains are invariant for all cells of the beam. Since the following relations are based on these nondimensional strains, they are also invariant with respect to cell number. Combining equations 4.2 , 4.3, and 4.4, we can solve for the nondimensional strain of one cable as a function of the other:

$$
\bar{\delta}_{\mathrm{rel}}=\frac{\bar{\delta}_{\mathrm{con}}}{1-\bar{\delta}_{\mathrm{con}}}
$$

Plotting the non-dimensional strains of the unit cell's cable contraction and release in figure 4.3 , we can clearly see a difference growing between the two as the cell deforms further. As the contraction cable's length is shortened at a constant rate, the release cable's length increases by an additional amount. The difference between 
these two strains is the geometric actuation deltd $2^{2}$ for which a modified antagonistic actuation system needs to compensate. We can formulate the following expression for the dimensionless geometric actuation delta between the contraction and release cables as a function of the contraction cable's normalized actuation:

$$
\bar{\Delta}_{\text {act }}=\bar{\delta}_{\text {rel }}-\bar{\delta}_{\text {con }}=\frac{\bar{\delta}_{\text {con }}^{2}}{1-\bar{\delta}_{\text {con }}}
$$

The description of induced cable strain arising from purely antagonistic actuation and the amount of $\bar{\Delta}_{\text {act }}$ needed for a modified antagonistic actuation system are equivalent: i.e. by quantifying the amount of induced strain, we automatically know the extra amount of cable release needed. Having an expression for $\bar{\Delta}_{\text {act }}$ opens up the possibility to design a non-circular actuation drum shape which will produce the modified antagonistic actuation necessary.

It should be noted here that in addition to accounting for geometrically-induced cable strain, a cam shape could account for other actuation resistance effects as well. Moored [5] indicates another mechanism counteracting actuation, termed prestress reorientation, which comes from the reorientation of force vectors in the vertical members of the tensegrity beam during actuation. The resistance force on a single unit cell due to this effect is given as:

$$
F_{\mathrm{pr}}=T_{0} \frac{\delta_{a}}{L_{0}}=T_{0} \bar{\delta}_{a}
$$

This force is directly proportional to the level of pretension and therefore could be quite substantial if the pretension is large. Meanwhile, the resistance force arising from the induced strain of $\bar{\Delta}_{\text {act }}$ can be calculated using Hooke's Law and equation 4.6 .

\footnotetext{
${ }^{2}$ The geometric actuation delta of the planar cross unit cell, $\bar{\Delta}_{\text {act }}$, should not be confused with the previously defined antagonistic actuation, $\delta_{\mathrm{a}}$, which uses a lower-case delta.
} 


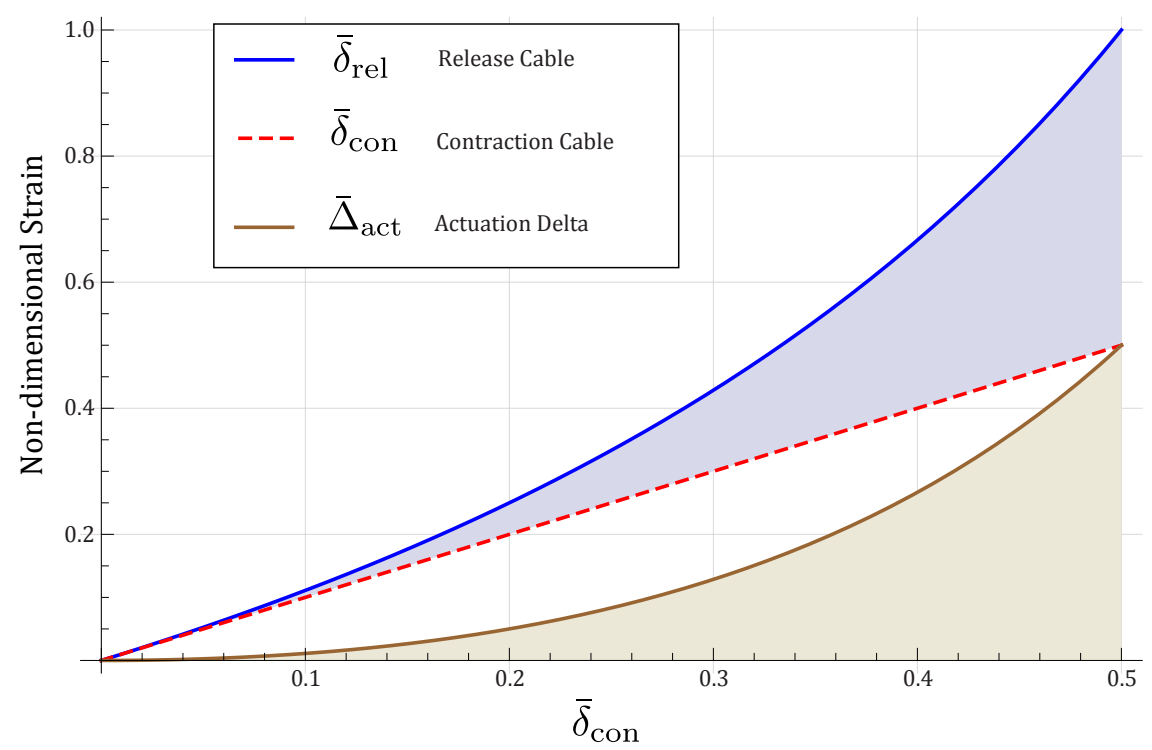

Figure 4.3: Plot of the release cable strain, $\bar{\delta}_{\text {rel }}$, as a function of the contraction cable strain, $\bar{\delta}_{\text {con }}$. As the contraction cable strains at a constant rate, the release cable strains at an increasing rate, governed by equation 4.5. The difference between these two strains increases with the tensegrity unit cell deformation and creates the actuation delta, $\bar{\Delta}_{\text {act }}$.

$$
F_{\Delta}=E_{\mathrm{c}} A_{\mathrm{c}} \bar{\Delta}_{\mathrm{act}}=\frac{E_{\mathrm{c}} A_{\mathrm{c}} \bar{\delta}_{a}^{2}}{1-\bar{\delta}_{a}}
$$

This force, $F_{\Delta}$, is caused by induced strain on the release cable (with crosssectional area $A_{c}$ and modulus $E_{c}$ ) during antagonistic actuation if the contraction cable is actuated by $\bar{\delta}_{a}$. It is clear this force will be large for relatively stiff structures, which are the focus of this study. For comparison of these two effects, we can take values from the tensegrity fin design example in [5]: $T_{0}=5 \mathrm{~N}, A_{c}=5.3 \times 10^{-8} \mathrm{~m}^{2}$, and $E_{c}=97 \mathrm{GPa}$. Using these values we calculate that $F_{\mathrm{pr}}=5 \bar{\delta}_{a} \mathrm{~N}$ and $F_{\Delta}=$ $5150 \bar{\delta}_{a}^{2} /\left(1-\bar{\delta}_{a}\right)$ N. Plotting these forces in figure 4.4 over a range of $\bar{\delta}_{a}$, we see that the actuation resistance from prestress reorientation is minuscule in comparison to that from the geometric actuation delta.

Small as it might be, if it were desired to account for the prestress reorientation 


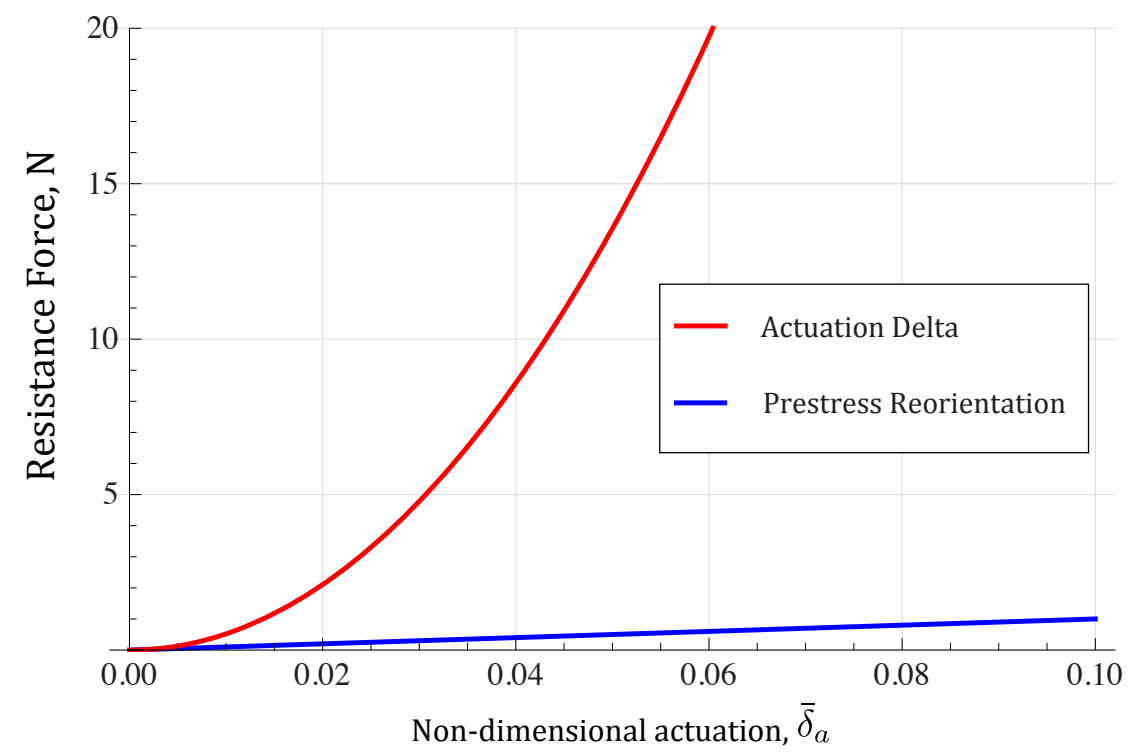

Figure 4.4: Plot of two contributions to actuation resistance. Clearly the resistance arising from the geometric actuation delta is much greater than that from prestress reorientation, for the relatively stiff structure in question.

effect, the amount of extra release, $\delta_{a}^{*}$, needed for a single cell is calculated as [5] :

$$
\delta_{a}^{*}=\delta_{a} \frac{2 T_{0}}{E_{\mathrm{c}} A_{\mathrm{c}}}
$$

The amount of extra release to relieve prestress reorientation resistance is proportional to the nominal actuation, $\delta_{a}$, and the proportionality is the inverse of the structure's relative stiffness, $k_{\mathrm{r}}$ (from section 3.1). Again, from this relationship we can see that for a high relative stiffness, the prestress reorientation effect diminishes in importance. Using the relative stiffness of the design example in [5] to put this into perspective, we see that $\delta_{a}^{*}=0.0019 \delta_{a}$. So if, for example, we wanted an actuation of $\delta_{a}=2 \mathrm{~cm}$ (representing $\bar{\delta}_{a}=0.24$ for the design example in [5]), the extra cable release would only be $\delta_{a}^{*}=39 \mu \mathrm{m}$.

Clearly this is a small effect and the amount of extra release is likely well below the manufacturing resolution that might be available. It can be concluded from 
this example that prestress reorientation is only a significant effect for relatively soft structures (and chiefly those with very high pretension), which are not the focus of study here, so we can continue designing the cam shape with disregard to this mechanism.

From the cable strains in figure 4.3 and from the actuation delta of equation 4.6, it is clear that the total actuation for the release cable needs to be larger than for the contraction cable. In the baseline actuation scheme of a circular drum, the actuation delta is zero and the absolute value of actuation for both cables wrapped around the drum is equal to the arc length swept through by the drum rotation angle: $s=r \theta=\delta_{a}(r, \theta)$. Clearly a larger radius increases the actuation amount for a given rotation, and a smaller radius decreases the actuation amount. From this baseline, we can imagine a cam-shaped actuation drum with a variable radius such that more or less cable will be released depending on the angular position of the drum, as shown schematically in figure 4.5 .

The main problem now is: how do we describe a cam shape, defined by $r(\theta)$, in terms of the necessary actuation delta? The actuation delta for the cam shape can be formulated as:

$$
\bar{\Delta}_{\mathrm{cam}}=\bar{s}_{\mathrm{rel}}-\bar{s}_{\mathrm{con}}
$$

Here, the arc length swept out by each side of the cam is normalized as $\bar{s}=$ $s / L_{0}$ (by the original length of a unit cell, as with previous normalizations) and the formulation of $\bar{\Delta}_{\text {cam }}$ is subtractive similar to equation 4.6 under the assumption that $s_{\text {con }}>0$. This actuation delta for the cam can then be described in terms of the cam shape if the arc lengths are related to $\theta$ and $r(\theta)$. In general, the arc length swept out by an arbitrary curvilinear path described by $r(\theta)$, can be given by [117]: 

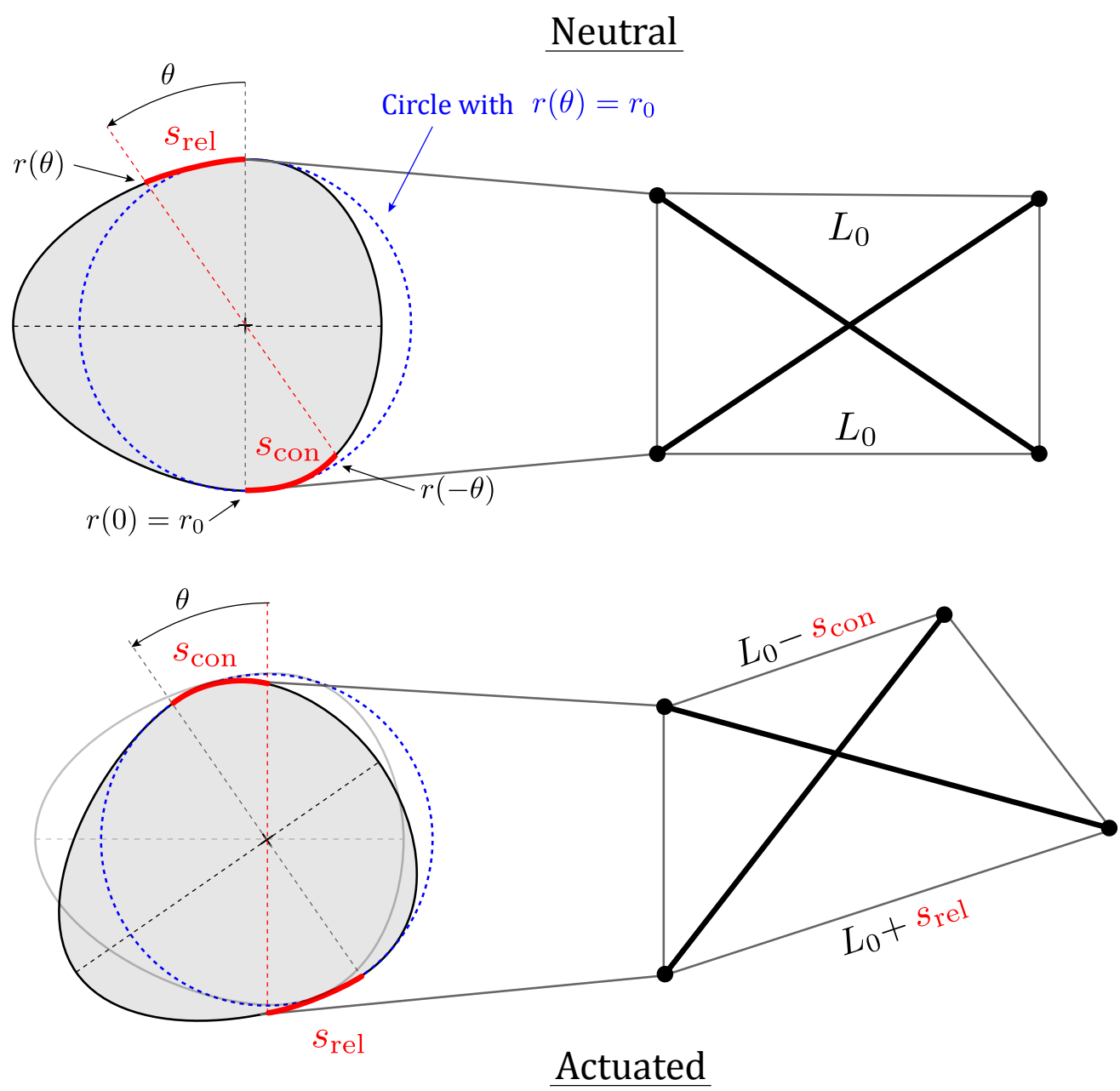

Figure 4.5: Schematic of cam concept, showing arc lengths on an actuation drum with varying radius $r(\theta)$. The cam shape is symmetric about the line from $\theta=\pi / 2$ to $\theta=-\pi / 2$. The radius increases on the release side of the cam, so that $r(\theta)>r_{0}$ for $0<\theta<\pi / 2$ and the radius decreases on the contraction side of the cam, so that $r(\theta)<r_{0}$ for $0>\theta>-\pi / 2$. This general shape ensures that $s_{\text {rel }}>s_{\text {con }}$. 


$$
s=\int_{0}^{\theta} \sqrt{r(\theta)^{2}+\left(\frac{d r(\theta)}{d \theta}\right)^{2}} d \theta
$$

It would be convenient to solve for the radius at any point, $r(\theta)$, in terms of the desired actuation delta using this relation, however there is no general closed-form solution. Instead we can start with an assumed form of the solution for $r(\theta)$, and try to solve for the parameters of the model. The simplest possible solution form to achieve our goal of matching the actuation delta would be a linear one, in which the radius varies linearly with $\theta$, such as: $r(\theta)=m \theta+r_{0}$. Again normalizing ${ }^{3}$ this relation by the unit cell length $L_{0}$ such that $\bar{r}(\theta)=r(\theta) / L_{0}, \bar{m}=m / L_{0}$, and $\bar{r}_{0}=r_{0} / L_{0}$ we have a simple dimensionless model for the radius as a linear function of $\theta$ :

$$
\bar{r}(\theta)=\bar{m} \theta+\bar{r}_{0}
$$

For this simplest model, a closed-form solution for the arc length can be found using a symbolic manipulation tool like Mathematica, but it is unduly complex and only valid for $\theta>0$. Taking a two-term series approximation of the solution about $\theta=0$ and solving for $\bar{m}$ in terms of $\bar{r}_{0}, \theta$, and $\bar{s}$ yields multiple explicit solutions, but again are extraordinarily complicated algebraically. A more straightforward approach for determining the appropriate value of $\bar{m}$ would be one in which the arc lengths for both sides of the cam are calculated numerically over a range of $\bar{m}$ so that $\bar{\Delta}_{\text {cam }}$ can be compared to the desired actuation delta, $\bar{\Delta}_{\text {act }}$.

Equations 4.11 and 4.12 indicate that the arc lengths should depend not just on the value of $\bar{m}$, but also upon $\bar{r}_{0}$. An appropriate value of $\bar{r}_{0}$ is chosen by considering the desired maximum tip amplitude of the tensegrity beam and approximating the

\footnotetext{
${ }^{3}$ When normalized as the cell actuations $\bar{\delta}$ above, this formula for radius also applies to multiplecell beams and is invariant to cell number for the same reason. This simplifies the analysis that follows, because it only needs to be done once for the whole beam, not for each cell. To be explicit, if the nominal drum radius corresponding to the $j$ th cell of the beam is $r_{j}=j r_{0}$ and if it is normalized by the length of cable for the $j$ th cell, then we see that $\bar{r}_{j}=r_{j} / L_{j}=j r_{0} / j L_{0}=r_{0} / L_{0}=\bar{r}_{0}$, which is invariant with respect to the cell number. By this normalization, the real radius with units does scale with cell number as $r_{j}=j \bar{r}_{0} L_{0}$ as does the value of $m_{j}=j \bar{m} L_{0}$.
} 
actuation giving rise to that tip amplitude by the arc length swept out by a circular drum of the same radius. Using equation 3.1 and the arc length of a circular drum, we can estimate an appropriate radius for an $\mathrm{MC}$ tensegrity beam as:

$$
\bar{r}_{0} \approx \frac{\bar{A} h}{\theta L}
$$

Here $\bar{A}$ is the tip amplitude of the beam normalized by the total beam length, $L$. This relation shows that for a fixed maximum $\theta$, the tip amplitude is proportional to both $\bar{r}_{0}$ and the beam aspect ratio $L / h$. Due to the symmetry of the cam, the absolute maximum $\theta$ is $\pi / 2$ but it is not necessary to use the entire surface of the cam. The maximum practical value for $\theta$ depends on the specifics of each application, such as the actuator being used to drive the rotation. Similarly, the radius $\bar{r}_{0}$ depends on the specifics of the application, so the value given by equation 4.13 is only an approximate value that would be appropriate, and different values could be used successfully. The radius given by 4.13 represents the minimum in order to achieve the desired amplitude using rotation $\theta$. A maximum radius is governed by physical considerations for the actuation system, and limits the maximum $\theta$ that can be used. For a typical biological amplitude of $\bar{A}=0.5$, a sample beam aspect ratio of $L / h=6$, and assuming $\theta_{\max }=\pi / 4$, we have $\bar{r}_{0}=0.1$. This value can be used for the following example of finding $\bar{m}$.

Proceeding with a candidate $\bar{r}_{0}$, we can make an array of values for $\theta$ (from 0 to $\theta_{\max }$ for $\bar{s}_{\text {rel }}$ and from 0 to $-\theta_{\max }$ for $\left.\bar{s}_{\text {con }}\right)$ and an array of possible values for $\bar{m}$. Note that $\bar{m}$ has a maximum practical value defined by the model of equation 4.12 ; for $\theta<0$ (i.e. the contraction side of the cam) there is a value of $\bar{m}$ sufficiently large such that the radius goes to zero. If we consider the limiting case at $\theta=-\pi / 2$ (at the line of symmetry for the cam), then $\bar{m}_{\max }=2 \bar{r}_{0} / \pi$. For $\bar{r}_{0}=0.1, \bar{m}_{\max }=0.064$. The case of $\bar{m}=0$ represents a circular drum, so we can search in the range from 0 to $\bar{m}_{\max }$.

For each candidate $\bar{m}$, the radius $\bar{r}(\theta)$ is calculated at every $\theta_{i}$ using equation 
4.12. Transforming these points describing the cam surface from polar coordinates to cartesian coordinates, we can calculate the arc length cumulatively as the Euclidean distance between each point:

$$
\bar{s}_{i}=\sqrt{\left(\bar{x}_{i+1}-\bar{x}_{i}\right)^{2}+\left(\bar{y}_{i+1}-\bar{y}_{i}\right)^{2}}
$$

The required actuation delta of the unit cell is calculated for each $\bar{m}$ with $\bar{s}_{\text {con }}$ acting as $\bar{\delta}_{\text {con }}$ using equation 4.6 , and the actuation delta of the cam shape is calculated using 4.10 . The error between these two actuation deltas at each point along the cam surface is calculated as:

$$
e_{i}=\left(\bar{\Delta}_{\mathrm{cam}, i}-\bar{\Delta}_{\mathrm{act}, i}\right)^{2}
$$

The total error for each candidate value of $\bar{m}$ is simply the integration of all $e_{i}$ over the range of $\theta$, so that $e_{\text {tot }}=\sum e_{i} d \theta$. The total error has been plotted for this example in figure 4.6. The minimum total error, which is $7.3 \times 10^{-13}$, occurred for $\bar{m}=0.0101$. Figure 4.7 shows the error between the actuation deltas (using this best $\bar{m}$ ) at each point along the cam, which is largest near $\theta_{\max }$. However the magnitude of even the largest error is quite small, at less than $4.5 \times 10^{-12}$. The fact that this error is small can also be seen in figure 4.8, where there is excellent agreement between the required actuation delta and the actuation delta produced by the cam shape.

From the arc lengths shown, it may seem like the actuation delta produced by the shape of the cam would be almost inconsequential. However consider that the actuation delta at $\theta_{\max }$ is more than 0.006 , which represents a cable strain of $0.6 \%$. Using $A_{c}$ and $E_{c}$ of braided stainless steel cable from the force estimations earlier in this section, that strain translates to more than $30 \mathrm{~N}$ of force, which is a substantial effort just to actuate an unloaded tensegrity beam. However, using the cam shape provided here, that unnecessary actuation resistance is eliminated. The final cam shape producing this actuation delta is shown in figure 4.9, with a circular drum of 


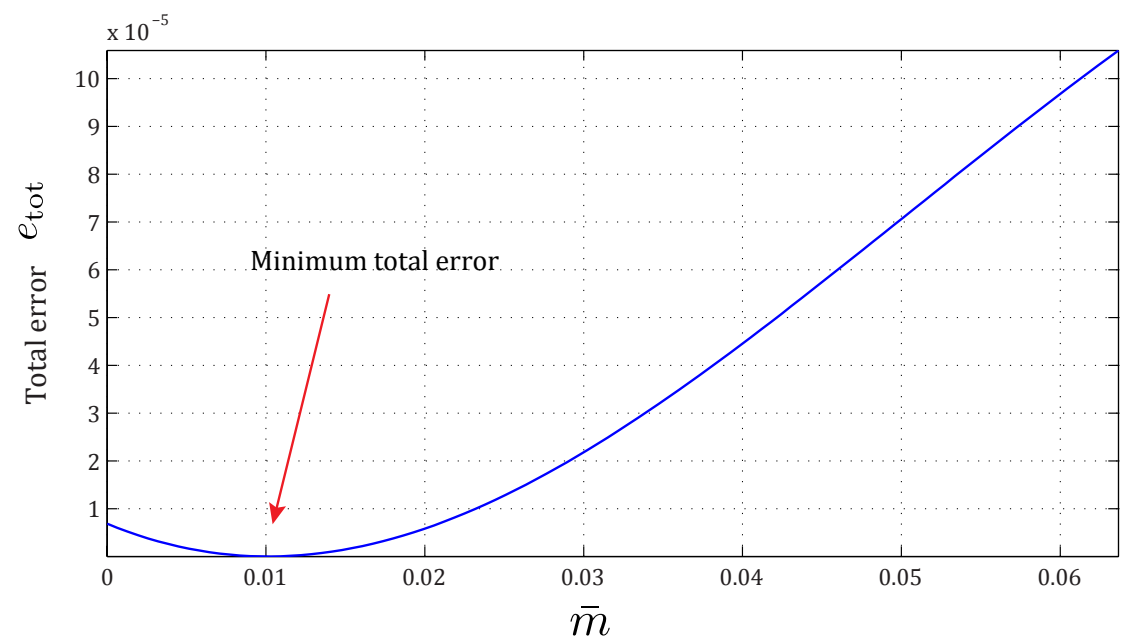

Figure 4.6: Minimum total error of the cam shape for target actuation delta, based on $\bar{r}_{0}=0.1$.

radius $\bar{r}_{0}$ shown for comparison.

The example of this particular cam shape using typical values of a tensegrity beam has been for the purposes of demonstrating the efficacy of the design method proposed, which eliminates induced strain when actuating. The same method can be carried out using different design parameters to similar effect. It is interesting to note however that the value of $\bar{m}$ does depend on $\bar{r}_{0}$. In order to determine the nature of the relationship between these parameters, the process outlined above was carried out for a wide range of $\bar{r}_{0}$ and for each case the optimal (minimum total error) value of $\bar{m}$ was found. The result is shown in figure 4.10. Clearly the value of $\bar{m}$ increases along with $\bar{r}_{0}$, however there is an apparent discontinuity around the value of $\bar{r}_{0} \approx 0.35$, after which the optimal value of $\bar{m}$ does not rise nearly as quickly. From the plot of total error (corresponding to each $\bar{m}$ ), we can see that soon after this transition, around $\bar{r}_{0} \approx 0.5$, the total error rises substantially. This indicates that for large enough values of $\bar{r}_{0}$, this model for a cam shape fails to provide the necessary actuation delta. Therefore in using this method values of $\bar{r}_{0}$ should be kept low, preferably below $\bar{r}_{0} \approx 0.35$. The cam design process for the active tensegrity beam used in the fin is carried out with this limit in mind. 


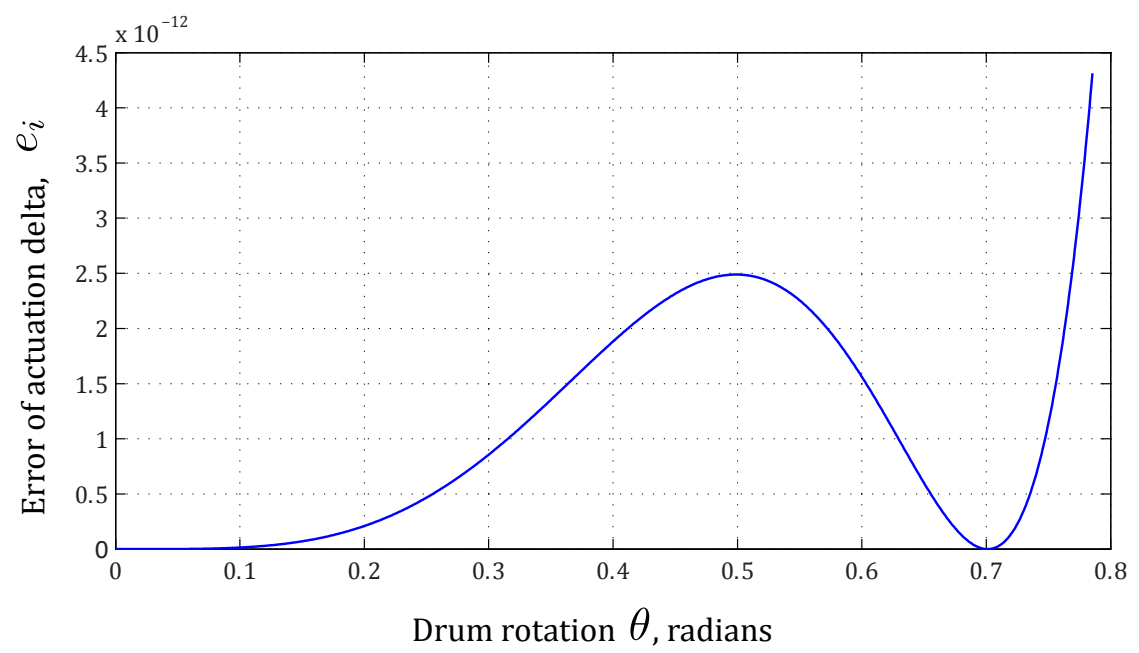

Figure 4.7: Actuation delta error along the surface of the cam for $\bar{r}_{0}=0.1$ and $\bar{m}=0.0101$. The error is largest at $\theta_{\max }$, but the magnitude of this error is very small. 

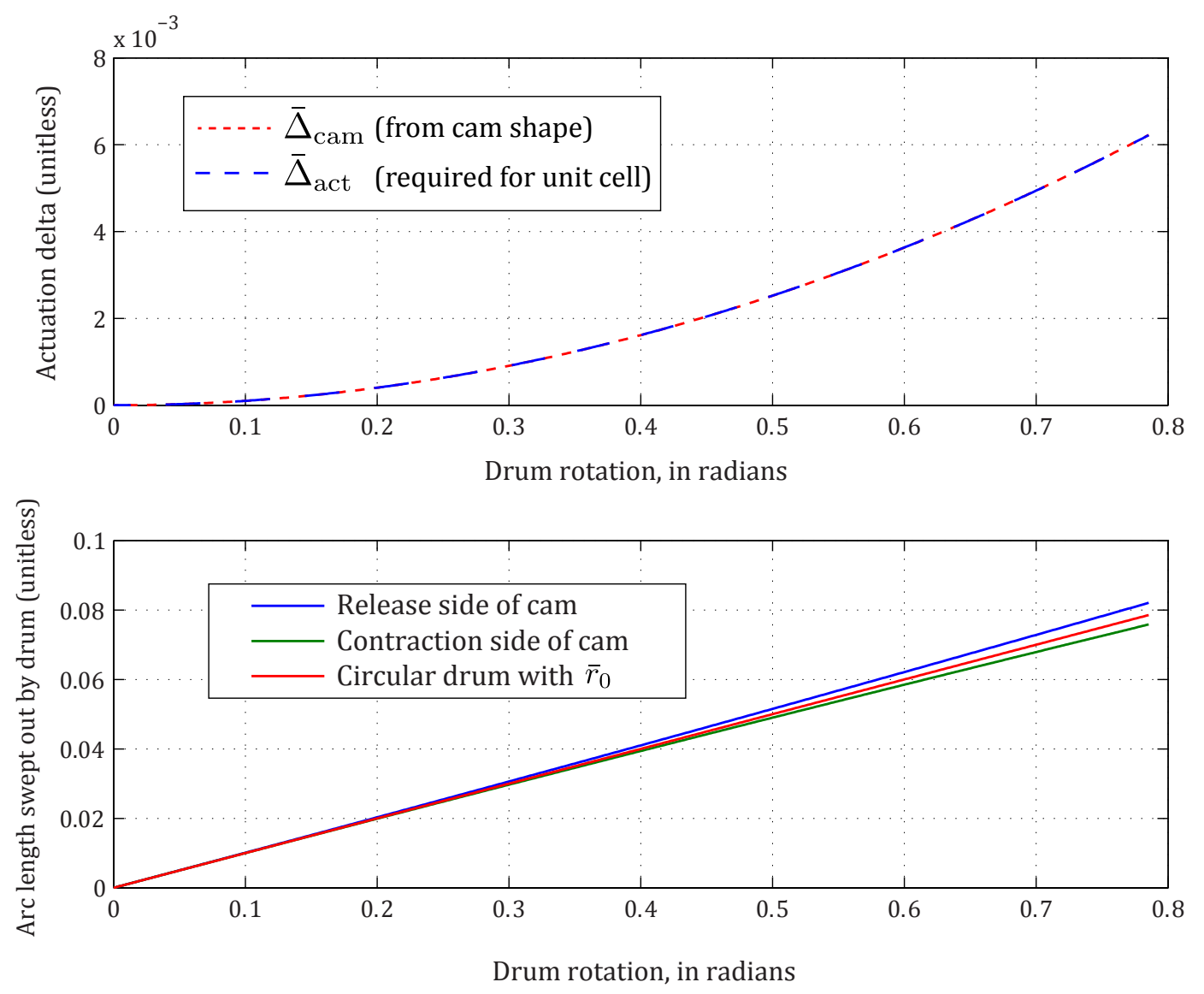

Figure 4.8: Resulting actuation delta for the cam with $\bar{r}_{0}=0.1$ and $\bar{m}=0.0101$, showing excellent agreement with the actuation delta required for the unit cell. Also shown are the cumulative amounts of arc length (corresponding to cable length changes) for both the release and contraction sides of the cam, with the arc length of a circular drum shown for comparison. 


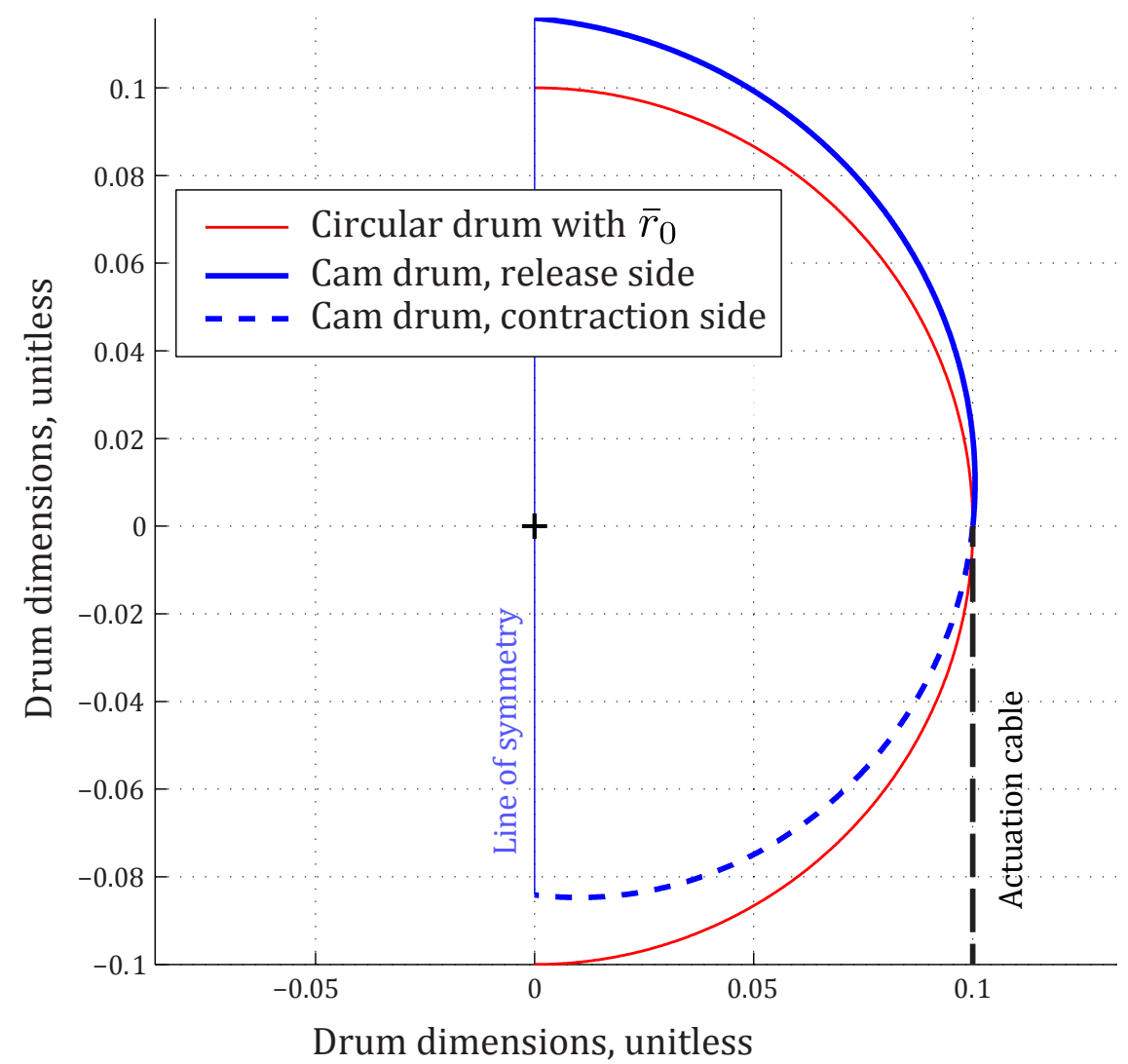

Figure 4.9: Resulting cam shape for $\bar{r}_{0}=0.1$ and $\bar{m}=0.0101$, showing the radius increasing with $\theta$ on the release side of the drum, and the radius decreasing on the contraction side of the drum. A circular drum with equivalent $\bar{r}_{0}$ is shown for comparison and the black line shows where an actuation cable would lie tangent on the drum. The complete actuation drum would simply be mirrored about the line of symmetry. 

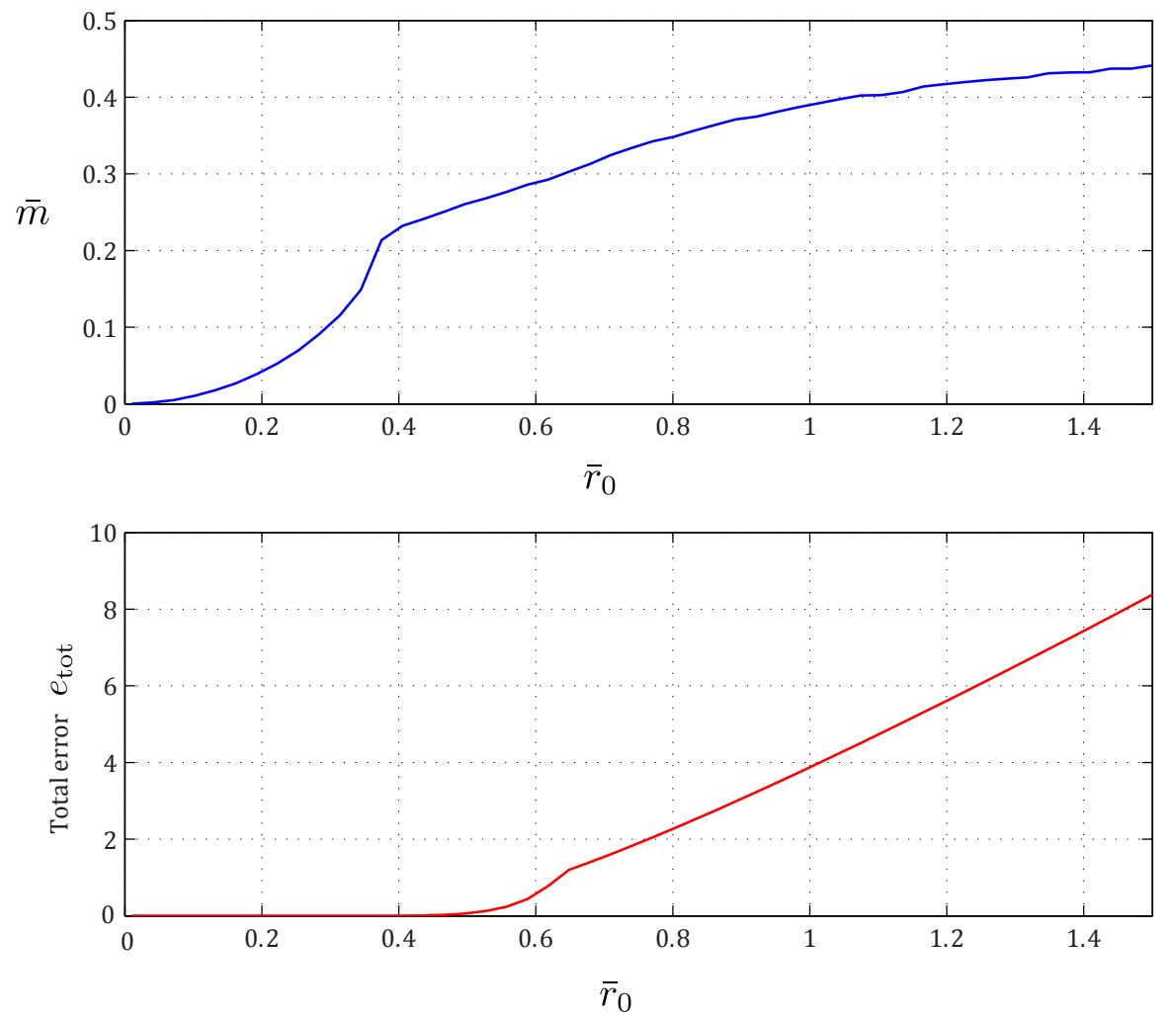

Figure 4.10: Optimum values of $\bar{m}$ plotted against $\bar{r}_{0}$. There is a transition in the relationship around $\bar{r}_{0} \approx 0.35$, after which the cam model fails to provide the actuation delta necessary, as shown by the rise in total error. 


\subsection{Fin Concept}

With validated tensegrity mechanics and a means of actuation to minimize induced cable strain for large amplitude beam bending, the next issue is the design of a fin which incorporates a tensegrity beam for the structural basis and is also capable of handling fluid loading to produce thrust. The overall idea is to implement the kind of tensegrity beams previously discussed in order to create kinematics similar to batoid rays. Since this is an open-ended design problem with many possible solutions, the following discussion serves to justify the design choices made for this study, not as the only or absolute best way to create batoid kinematics using tensegrity.

The goal of this fin design is to produce the major components of oscillatory batoid ray kinematics - i.e. large span-wise bending (oscillatory flapping) with some amount of chord-wise curvature (an undulatory wave) - using the simplest arrangement of tensegrity beams possible. Considering the number of design choices that must be taken into account, it seems that increasing complexity obscures us from the objective of studying batoid swimming performance. Therefore it is suggested here that a fin using only one beam near the leading edge of a compliant skin could accomplish the objective, creating the appropriate kinematics and allowing us to quantify swimming performance.

It should be noted that there has been a recent proposal [5] to use a series of tensegrity beams actuated in sequence to actively produce both the span-wise bending and the chord-wise traveling wave components of batoid kinematics. This overall design choice presents two problems though: first, the level of difficulty for both constructing and controlling the entire structure scales with the number of beams; second (and more importantly), this design could lead to large amounts of energy wasted on straining an artificial skin. If multiple beams are actuated with a phase difference to create a traveling wave, then the distance between adjacent beams would be constantly changing, an effect most extreme at the beam tips. The result is that a 
skin material spanning across these beams would undergo significant strain $\rrbracket^{4}$ the level of which would be determined by the specific kinematics of the beams (amplitude and phase difference). Straining the skin material would not contribute to thrust production and would be a waste of input energy so for these reasons a multiplebeam approach is not taken here.

The active tensegrity beams covered in the previous chapter can produce the large span-wise bending desired, but some means of producing chord-wise curvature with only one beam is still needed. Looking towards biology for inspiration, there are two notable observations: fish and most biological swimmers in general are highly flexible [68, 118]; and the musculature of batoid rays is heavily concentrated towards the leading edge of the fin (see figure 4.12), leaving far less muscle for active fin deformation in the distal and posterior portions of their fins. It therefore seems likely that the full kinematics seen on the fins of biological rays are a product of both active and passive mechanisms ${ }^{6}[61$, 119]. The assumption then is that a simple design can produce the appropriate kinematics by using a tensegrity beam near the leading edge to actively deform for large span-wise flapping amplitude and a compliant skin to passively deform for chord-wise curvature. Several recent bio-inspired propulsor studies [4, 41, 48, 120] have used a general design in which some mechanism is embedded in a compliant polymer skin in order to form a smooth, streamlined propulsive surface for hydrodynamic loading $]^{7}$ — an approach that will be adopted here.

With a general concept of the fin in place, the next item to resolve is a planform

\footnotetext{
${ }^{4}$ This skin straining scenario could possibly be avoided by using some kind of flexible corrugated or baffled material for the skin, which would easily accommodate the strain. However this type of design is undesirable because it would not allow for a smooth, streamlined airfoil shape seen in actual ray fins.

${ }^{5}$ Unless the strain energy could be released in some way to excite a resonance of the structure, which would further complicate the design process. Taking advantage of structural resonance is an important issue to consider, but is beyond the scope of this study, which is based on reproducing kinematics.

${ }^{6}$ But this is a subject of study for biologists and currently there are no definitive answers on this pertaining to batoid rays.

${ }^{7}$ At the extreme edge of simplicity, other recent studies have shown that highly flexible panels actuated only at the leading edge can be used to produce thrust [121].
} 
for this fin, which defines the chord length as a function of span position, and thus determines the overall fin aspect ratio as well. Variations in planform and aspect ratio certainly exist amongst batoid rays, but the overall morphology of oscillatory rays is remarkably consistent and the general fin planform shape is triangular with differing levels of sweep [58]. The influence of swept-back tips and other planform variants is not well-studied for this type of swimming so the default planform shape for this study is chosen to be the simplest possible, which is purely triangular. While this may not seem very representative of biology, the pectoral fin of the spotted eagle ray (Aetobatus narinari) is indeed very nearly an isosceles triangle, as shown in figure 4.11. This planform is simple and does not confuse the design with unknown effects of swept tips or other features, therefore it will be used for this study.

Aspect ratio for the body of a ray can be defined as $A R_{\text {body }}=b^{2} / S$, where $b$ is the total tip-to-tip wingspan and $S$ is the total area, including the rigid body. Since the bodies of oscillatory rays are rhombic in planform, the area can be approximated as $S \approx b c_{\mathrm{BL}} / 2$, where $c_{\mathrm{BL}}$ is the chord length of the body (not including the tail), which makes the aspect ratio $A R_{\text {body }} \approx 2 b / c_{\mathrm{BL}}$. Typical values of this body aspect ratio for oscillatory batoids are in the range of $3.2-3.5$ [58]. To convert this body aspect ratio to an aspect ratio for the fin alone, it will be assumed that $A R_{\text {fin }}=A R_{\text {body }} / 2$, since the span of a fin is approximately half of the total wing span. A value of $A R_{\text {fin }}=1.65$ (which corresponds to $A R_{\text {body }}=3.3$ ) will be used for the design of this fin moving forward, creating a fixed relationship between root chord and span such that $c_{\text {root }}=1.22 b$.

As mentioned above, the tensegrity beam should be positioned near the leading edge of the fin for two reasons: we see in oscillatory batoid rays the majority of the musculature toward the leading edge, and the objective of a passive traveling wave on the fin will only be accomplished if the flexible portion of the fin trails the active tensegrity beam. However with this information alone it is unclear where exactly to position the base of the tensegrity beam along the root chord and what 


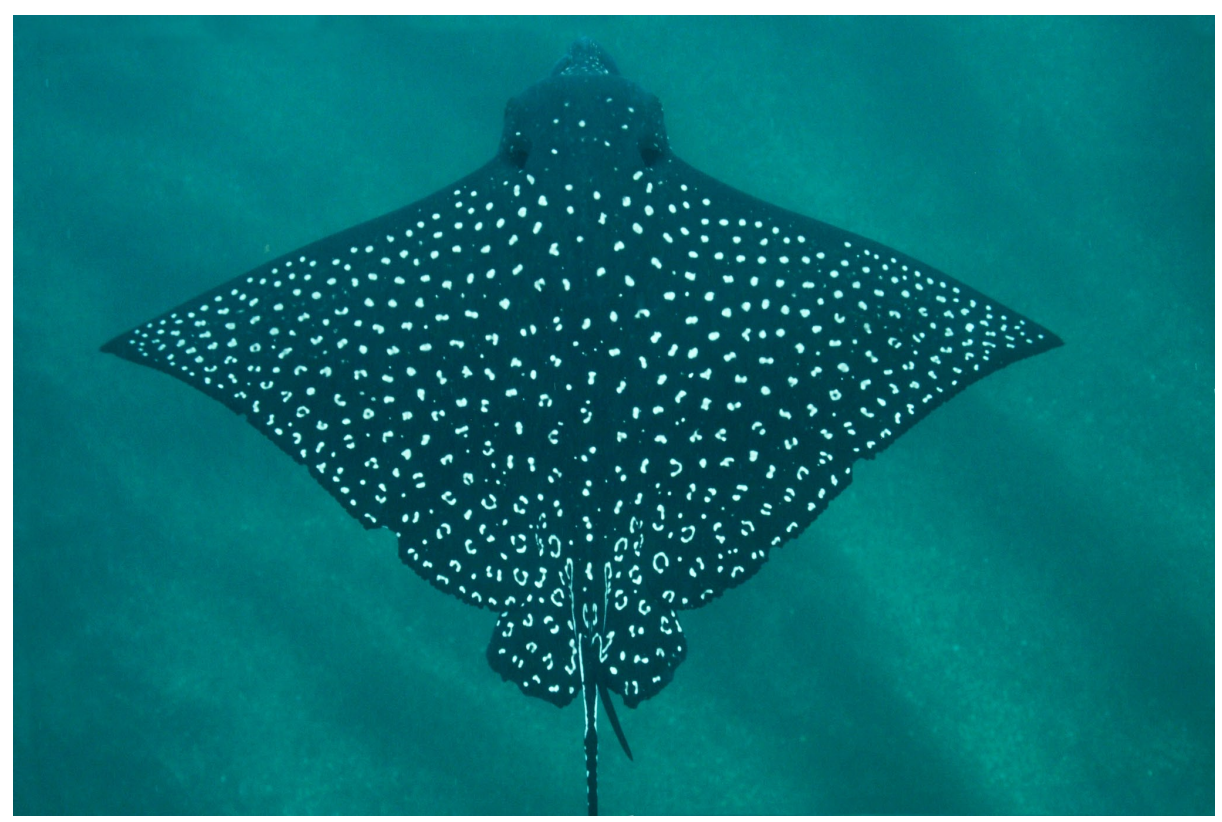

Figure 4.11: Spotted eagle rays, another common myliobatoid species, exhibit a remarkably triangular fin planform (with very little sweep or other curvature). Image source: [122].

angle the beam should make relative to the span line (or $x$-axis, perpendicular to the stream-wise direction). We can determine both of these parameters simultaneously by considering two things: the fineness ratio, which is the ratio of chord length to maximum thickness (inverse of thickness-to-chord ratio), is large for biological fins in general, essentially meaning they are streamlined; and that a tensegrity beam of finite minimum thickness must fit within this envelope. Therefore, in order to maximize the fineness ratio it is desirable to place this tensegrity beam of minimum thicknes: 8 along the thickest chord-wise position of the fin. The fineness ratio for myliobatoid rays is in the range of about 5 to 10 (meaning a thickness-to-chord ratio of 0.2 to 0.1) [58], so this gives a target for the design of the artificial fin.

To determine the portion of the fin with maximum thickness, consider that the cross sections of batoid ray fins (and of most biofoils in general) are very similar to

${ }^{8}$ The mechanics of section 3.1 demonstrate that reducing the height of the beam (corresponding to thickness of the fin) while keeping its length fixed reduces the beam's bending stiffness. However it is important to match the shape of a biological fin, so minimum height is desired. Fortunately, the reduction in bending stiffness due to increased beam aspect ratio can be compensated for simply by using stiffer (larger diameter) cable elements. 


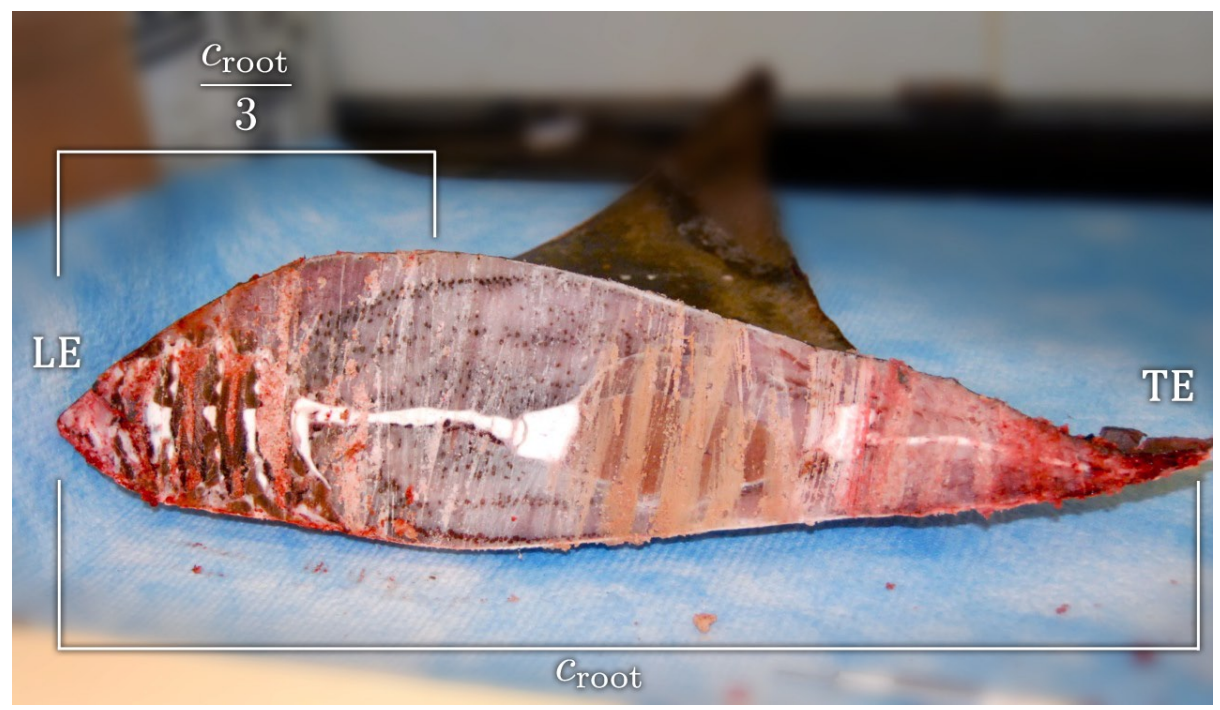

Figure 4.12: Chord-wise slice through the pectoral fin of a cownose ray at the fin root. Dissection clearly shows musculature concentrated towards the leading edge (LE) and relatively little towards the trailing edge (TE), resulting in an airfoil-like shape. Like a NACA airfoil, the thickest portion of the biofoil occurs around $1 / 3$ of the chord length away from the leading edge.

modern airfoils used in fixed-wing aircraft. Take for example a chord-wise cut through the fin of a cownose ray, shown in figure 4.12 , which is clearly reminiscent of a typical airfoil. Noting this, we can simplify our design by using common airfoils to create the cross-sectional shape of the fin. Standard four-digit NACA foils? have a maximum thickness at 0.3 chord lengths away from the leading edge (or approximately $c / 3$ ). This distance away from the leading edge at which maximum thickness occurs can be used as the line along which the tensegrity beam lies, thus simultaneously fixing its base position along the root chord and its angle with respect to the span line. For the isosceles triangular planform, the line of maximum thickness along which the beam should lie is tilted backwards (towards the trailing edge) relative to the span line $(x$-axis $)$ at an angle of $\phi=\tan \left(c_{\text {root }} / 6 b\right)$, as shown in figure 4.13 .

A major constraint on further choices for physical design of the fin is on overall size. The fin will undergo hydrodynamic testing in a water tunnel (described further

\footnotetext{
${ }^{9}$ Note that four-digit NACA foils have a format $c c t t$ where $c c$ denotes the amount and location of maximum camber, while $t t$ gives the thickness-to-chord ratio as a percentage. For example, NACA 0015 is an uncambered foil with $t / c=0.15$. Only uncambered foils will be used here.
} 


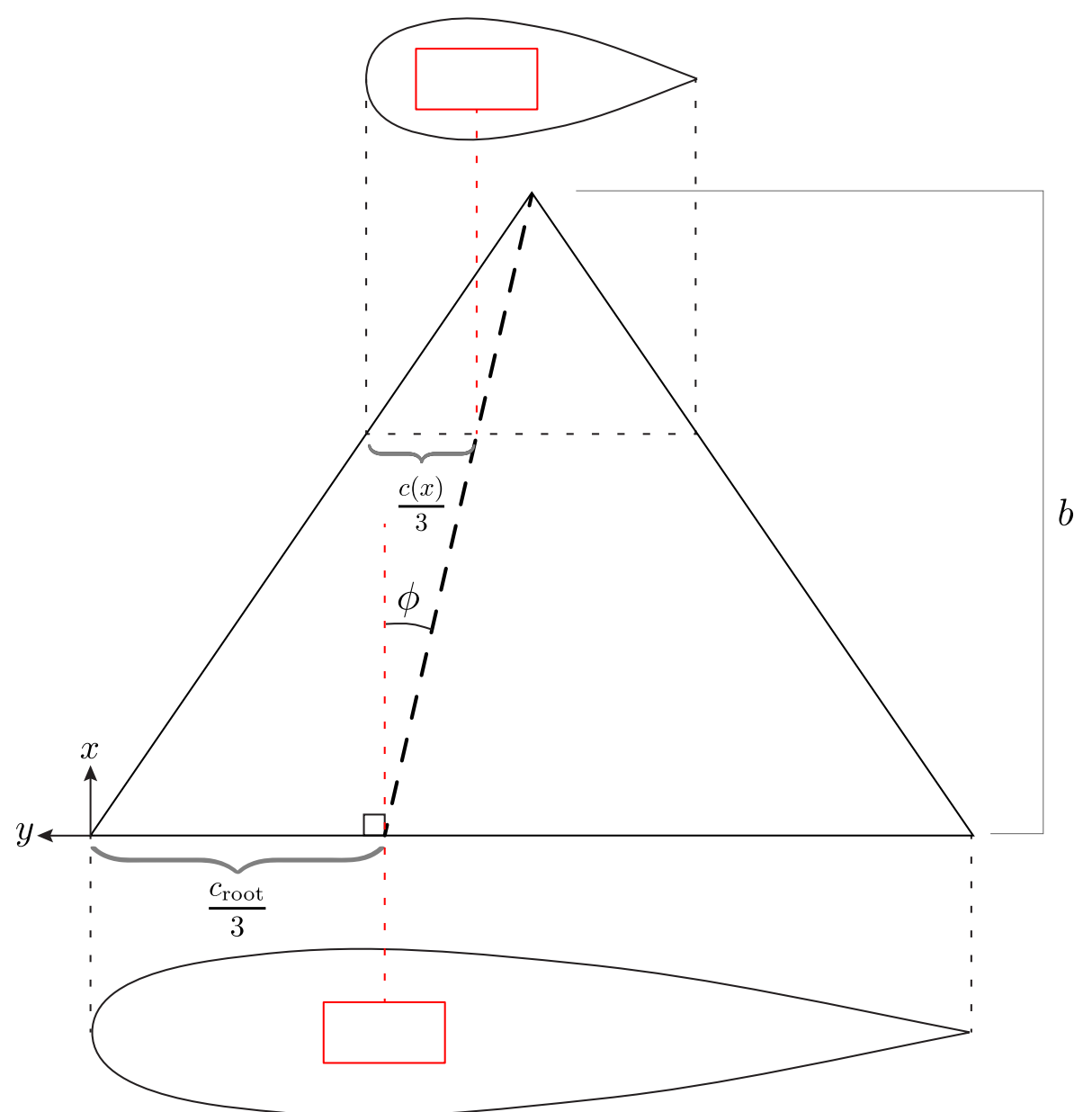

Figure 4.13: Schematic of triangular planform, showing the line of maximum thickness along which the tensegrity beam should ideally lie, at angle $\phi$ with respect to the spanwise direction ( $x$-axis). The foil at the root section here is a NACA 0020 and the foil at $c(x)$ is a NACA 0040, demonstrating the need for a lower fineness ratio approaching the tip to accommodate a beam of constant thickness (shown in red). 
in section 5.1), and therefore must fit within a fixed-size test section, which is 15 " $(38.1 \mathrm{~cm})$ wide and $20 "(50.8 \mathrm{~cm})$ deep. As mentioned in section 2.2.1 the maximum tip amplitude of batoids is typically in the range of $50 \%$ of the span length. Therefore the absolute maximum span for a fin flapping in this water tunnel would be 15", so that the tip-to-tip flapping amplitude just matches the tunnel width. The span length of the fin was chosen to be $8 "(20.3 \mathrm{~cm})$, so that the minimum distance between the fin tip and tunnel walls is $88 \%$ of the maximum tip amplitude $(\mathrm{A} / \mathrm{b}=0.5)$, in order to avoid flow interaction with the tunnel boundary as much as possible while still keeping the fin at a reasonable size for manufacturability.

The effects of wall proximity on unsteady flapping performance have not been well-documented in the literature, although two recent studies offer some insight on unsteady propulsion near a solid boundary. Quinn et al. [123] performed hydrodynamic experiments with a rigid airfoil, pitched at the leading edge while the distance from a solid boundary was varied. They found that at an optimal distance from the wall, thrust could be enhanced by as much as $40 \%$, however within experimental error, the measured efficiency was independent of distance from the wall. In another study, Quinn et al. [124] tested the propulsive performance of various thin flexible panels that were actuated through a heaving motion at the leading edge and varied the distance of these panels to a solid boundary. The results indicated that for certain frequency ranges thrust could be increased with proximity to the solid boundary. For the most rigid panel, this increase in thrust came at the cost of more power, and as a result the efficiency was independent of distance from the wall. For the more flexible panels, some increases in efficiency were observed when either resonant heaving modes of the panels were excited or when torsional modes of the panels were suppressed. Self-propelled swimming tests showed that swimming speed could be increased with wall proximity (especially for the stiffest panel), however swimming economy appeared to be completely independent of wall proximity for all panels. 
Therefore while there has been an attempt in this study to minimize wall effects ${ }^{10}$, it is assumed here that the impact of any such effects upon hydrodynamic results should be minimal, so long as the measures of performance include power consumption (i.e. efficiency and economy).

With the span and fin aspect ratio determined, this gives a root chord of $c_{\text {root }}=$ $9.75 "(24.8 \mathrm{~cm})$. For the triangular planform with these values of $b$ and $c_{\text {root }}$, this gives a beam angle with respect to the span line of approximately $12^{\circ}$. The amount of detail about the fin geometry determined so far is sufficient to estimate the overall fluid loading on the fin, while the remaining attributes of the fin geometry (such as fineness ratio at various span-wise locations) are based on features of the active tensegrity beam (beam length and minimum beam height), which are dictated by hardware considerations and are the subject of section 4.4 .

\subsection{Fluid Loading Model}

In order to determine necessary pretension levels in the structure to avoid a critical slackening condition, some estimate of external loading on the fin is required. Additionally, this loading estimate will dictate both the selection of an appropriate actuator to drive the active tensegrity beam and also the sizing of components (either based on minimum bending stiffness to allow a maximum deflection, or to avoid component failure). Estimating dynamic fluid loading on a surface with unsteady motion is no simple task, but an approach is laid out here which gives a rough estimate in order to obtain the order of magnitude for these forces. Let us assume a simple motion for the fin to approximate flapping, which is given by a rigid plate (with the triangular planform described previously) rotating about the root chord line, as shown in figure 4.14.

The forces on this rotating fin can be analyzed as drag perpendicular to a flat

\footnotetext{
${ }^{10}$ Through the choice of fin size, limited by the constraints of both the water tunnel and manufacturing capabilities.
} 


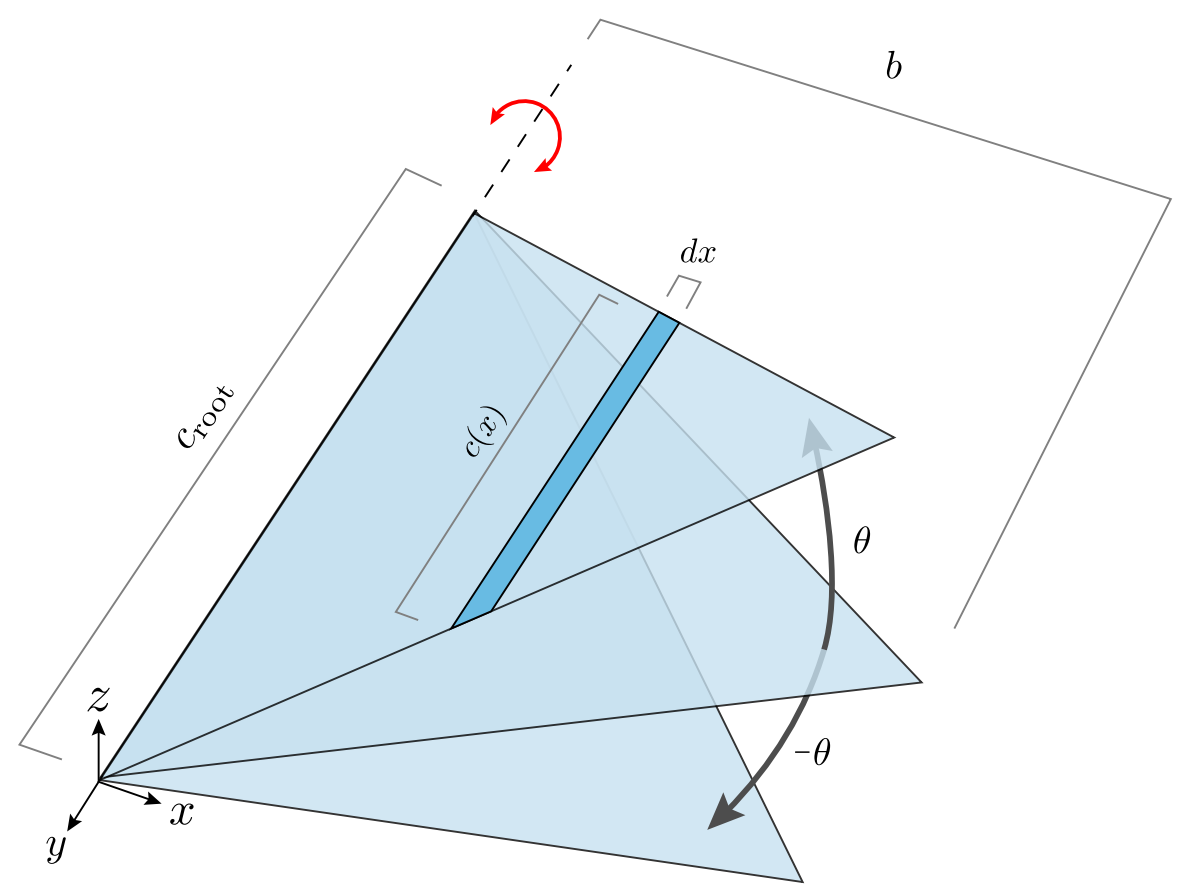

Figure 4.14: Schematic showing fin model used for fluid loading estimate. The fin is simplified to a flat, rigid triangular planform which rotates about the root chord.

plate by taking into account the dynamic pressure resulting from local flow velocities stagnating. Additionally, using a coefficient of drag, $c_{d}$, for a finite flat plate takes into consideration forces measured experimentally in steady flow, which are higher than only the forces expected for pure flow stagnation, due to suction on the back side of the plate and other intricacies of the flow ${ }^{11}$. Reported values for $c_{d}$ on a flat plate perpendicular to flow can vary widely, depending on experimental conditions, aspect ratio of the plate and other conditions. To be conservative in the estimate of force on the fin (erring higher), a high value of $c_{d}=1.98$ will be used [32. The total force on the plate is then given by the dynamic pressure multiplied by the fin area, both of which are integrated over the span of the fin:

\footnotetext{
${ }^{11}$ Note that this analysis does not take into account effects such as added-mass inertial loading or vortex interactions, which are both complicated and beyond the scope of this analysis. It is also worth noting that added-mass inertial forces should be maximal when the fin is accelerating near the maximum amplitude for each-half cycle of flapping, while the dynamic pressure forces should be maximal when the fin is passing through the neutral plane at maximum velocity. Since these effects don't occur simultaneously, they should not be additive.
} 


$$
F_{\text {plate }}=\frac{1}{2} c_{d} \rho \int_{0}^{b} U_{\max }(x)^{2} c(x) d x
$$

Here $\rho$ is the density of the fluid, $U_{\max }(x)$ is the local fluid velocity induced by flapping motion at some $\operatorname{span} x, c(x)$ is the chord length of the fin at $x$ and $b$ is the maximum span. The chord length at any $x$ for the isosceles triangle planform is given by:

$$
c(x)=c_{\text {root }}\left(1-\frac{x}{b}\right)
$$

If we assume the fin is flapping sinusoidally by sweeping through angle $\theta$ with with frequency $f$ and amplitude $\theta_{\max }$, then the angular position at any time is $\theta(t)=$ $\theta_{\max } \sin (2 \pi f t)$ and the angular velocity is thus $\dot{\theta}(t)=2 \pi f \theta_{\max } \cos (2 \pi f t)$. The velocity at some point along the span normal to the fin surface is given by $U(x, t)=x \dot{\theta}$. The maximum fluid loads are of interest and we expect these to occur at maximum fin velocity, so we can express the maximum velocity normal to the fin surface as:

$$
U_{\max }(x)=2 \pi f x \theta_{\max }
$$

Thus by combining equations 4.16, 4.17, and 4.18 we have an expression for the total force on the plate:

$$
F_{\text {plate }}=2 \pi^{2} c_{d} \rho f^{2} \theta_{\max }^{2} c_{\text {root }} \int_{0}^{b} x^{2}-\frac{x^{3}}{b} d x
$$

Integrating over the span gives the maximum drag-based force over the entire fin as it passes through the neutral plane:

$$
F_{\text {plate }}=\frac{\pi^{2}}{6} c_{d} \rho f^{2} \theta_{\max }^{2} c_{\text {root }} b^{3}
$$

However it proves interesting to examine the distribution of the force per unit 


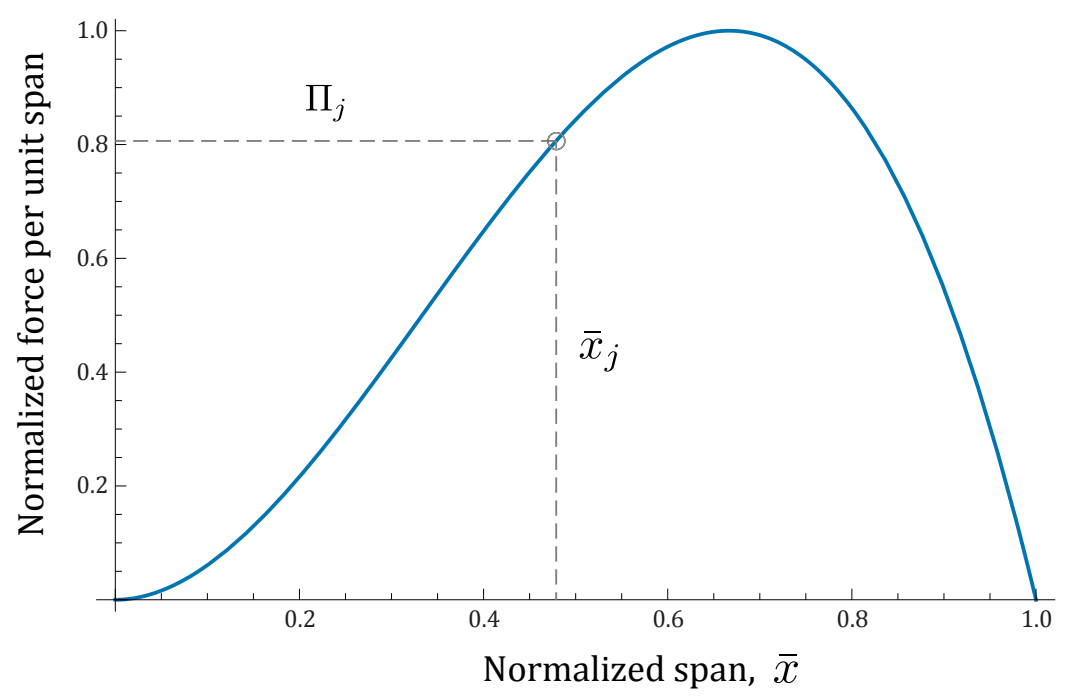

Figure 4.15: Plot of force per unit span from dynamic pressure, normalized by maximum value. Values of force per unit span, $\Pi_{j}$ can be determined from this distribution at each position $\bar{x}_{j}$, in order to estimate loading contributions, using equation 4.21 .

span arising from dynamic pressure, given by the integrand of equation 4.19 and shown in figure 4.15. This plot demonstrates the variation in force over the span length, based on the chosen planform and assumed velocity profile for flapping. Thus different planforms and flapping patterns would produce different distributions. The maximum force per unit span occurs at $x=\frac{2}{3} b$. As should be expected, this value drops to zero both at the fin root (because the velocity goes to zero) and at the fin tip (because the loading area goes to zero).

This distribution can be used to estimate how the loading distribution over the surface of the fin would translate to point loads on the nodes of a tensegrity beam embedded in the fin. If we look at the force per unit span at these nodal locations, we can estimate the relative force contribution that will act at each cell of the tensegrity structure. The normalized force per unit span at each node location (normalized by span length) $\bar{x}_{j}$ is $\Pi_{j}$. We can renormalize these contributions relative to each other and assume they represent the distribution of the total force so that the force at each node, $P_{j}$, is: 


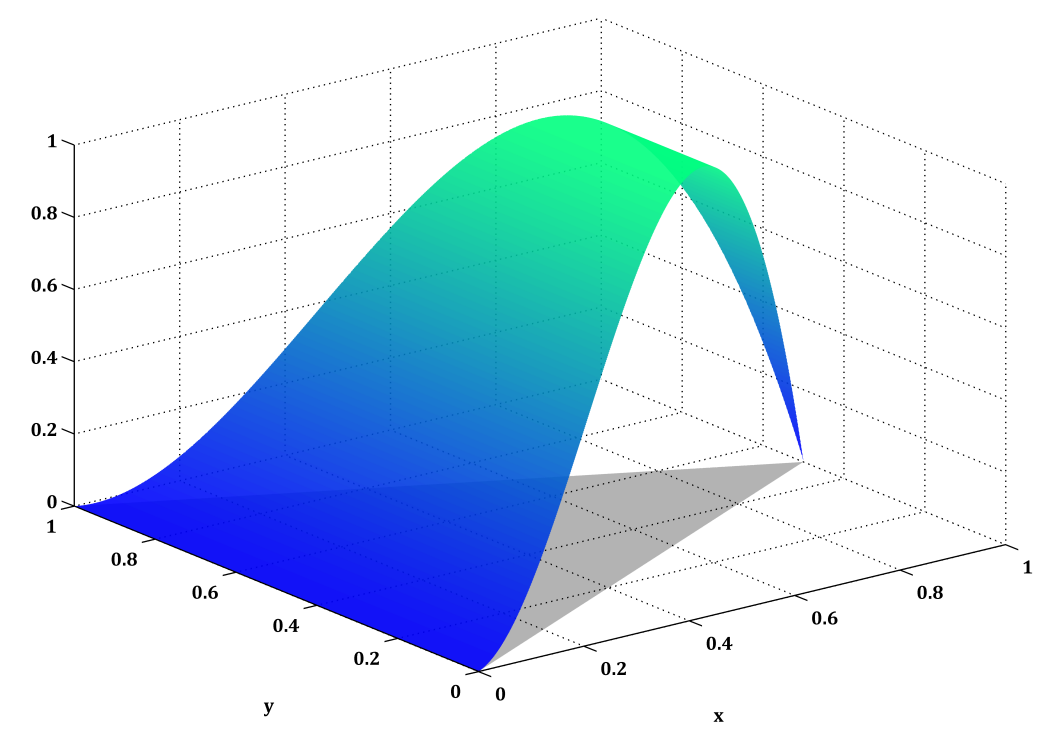

Figure 4.16: Fluid loading of equation 4.19 visualized on a triangular planform fin.

$$
P_{j}=\frac{\Pi_{j}}{\sum \Pi} F_{\text {plate }}
$$

The loading analysis of section 3.1 (specifically using equation 3.2) can be extended to account for these distributed forces at each node of an MC tensegrity beam. By simply taking the superposition of the change in tension for the cable of the $j$ th cell, $\Delta T_{j}$, caused by each load $P_{j}$, we can derive an expression for the total change in tension for the $j$ th cable:

$$
\Delta T_{j, \mathrm{tot}}=\frac{L}{2 N h}\left(P_{j}+2 \sum_{i=j+1}^{N} P_{i}\right)
$$

One implication of this result is that the change in cable tension will be highest in the first cell, since it is the only cell affected by all of the distributed loads. Therefore the criterion for cable failure due to external loading should be based on this first cell. Additionally, the optimal pretensions defined previously for a single tip load are no longer valid for this distributed loading scenario and instead the pretension 
distribution will depend upon the values of each $P_{j}$.

To actuate an MC beam against this distributed loading using a drum with radii $r_{j}=j r_{0}$, the total actuator torque required to produce this flapping would be:

$$
\tau_{\text {tot }}=r_{0} \sum j \Delta T_{j, \text { tot }}
$$

In summary, a methodology for estimating the fluid loading on the flapping fin is described, so that actuators and other components can be sized appropriately. The total loading and distribution on the nodes of the tensegrity beam will depend on final fin and beam parameters, which are determined in the following section.

\subsection{Implementation of an Active Tensegrity Beam}

Having laid out the framework for tensegrity design, the general concept of the robotic fin, and a method to estimate the fluid loading, the design and implementation of an active tensegrity beam can be completed. This beam will act as the structural basis for leading-edge span-wise bending in the artificial fin. As with the tensegrity mechanics experiments of section 3.2 , the number of cells was chosen to be 3 . Three cells give adequate shape resolution for the span-wise bending mode while minimizing complexity of design and fabrication.

Similar to the tensegrity structure used for load-displacement experiments, ball bearings are needed at the ends of each strut to create nodes with low-friction rotation. The diameter of these ball bearings is the limiting factor for the height of the beam, since the beam must be at least two ball bearing diameters tall, plus the width of strut material containing them, plus clearance for the vertical strut to rotate. The smallest flanged extended inner ring ball bearings that could be readily obtained as off-the-shelf items have an outer diameter of $0.25 "(6.35 \mathrm{~mm})$ and a flange diameter of $0.296 "(7.52 \mathrm{~mm})$. The result is a minimum strut height of approximately 0.75 " $(1.9 \mathrm{~cm})$, which is shown in figure 4.19 . 
Allowing for a minimum skin material thickness at any point along the beam of about $0.3 "(7.6 \mathrm{~mm})$, this gives a minimum fin thickness of $1.35 "(3.43 \mathrm{~cm})$. At the root chord, we then have a maximum possible fineness ratio of 7.2 . If this minimum thickness remains constant, the fineness ratio will decrease moving outwards spanwise (as the chord length decreases). In order to avoid a very small fineness ratio at the distal portion of the fin (approaching a bluff body), the tensegrity beam must terminate before the maximum span length of the fin so that the thickness can taper down. A final tensegrity beam length of $5 "(12.7 \mathrm{~cm})$ was chosen to give a minimum fineness ratio of about 2.5 just beyond the terminal node of the beam.

Using these size constraints, a 3D model of the fin can be created. To accommodate the variable fineness ratio along the span, several NACA foils were placed at various span-wise locations, and the solid shape is created by lofting between the foil sections, as shown in figure 4.17. Fineness ratio is maximum at the root, then decreases towards the beam tip as the maximum thickness remains roughly the same, but the chord length decreases. Past the beam tip, the fin tapers down and fineness ratio increases again. The distal end of the fin ends in a small foil section with chord length $0.5 "(1.3 \mathrm{~cm})$, so it is not exactly triangular in planform (actually rhombic instead). This is done to simplify both 3D modeling and the skin molding process.

With a beam 5" long swept back at an angle of $12^{\circ}$, the 3 cells have nodes at the span-wise locations of $x_{j}=[0.204,0.408,0.611] b$. Using the values of $\Pi_{j}$ at these locations (given by the integrand of equation 4.19) the distribution of forces are estimated as $P=[0.12,0.36,0.52] F_{\text {plate }}$. So by this method, about half of the total loading on the fin will be applied at the beam tip, with the rest distributed to the other two cells, in decreasing amounts away from the tip.

To complete the estimate of $F_{\text {plate }}$, the parameters of $f$ and $\theta_{\max }$ are still needed. Biological observations of oscillatory rays demonstrate maximum flapping frequencies of about $1.5 \mathrm{~Hz}$ [54], so for the purposes of this study, the maximum flapping frequency (where maximum dynamic loading is expected to occur) will be $f=1.5 \mathrm{~Hz}$. The non- 


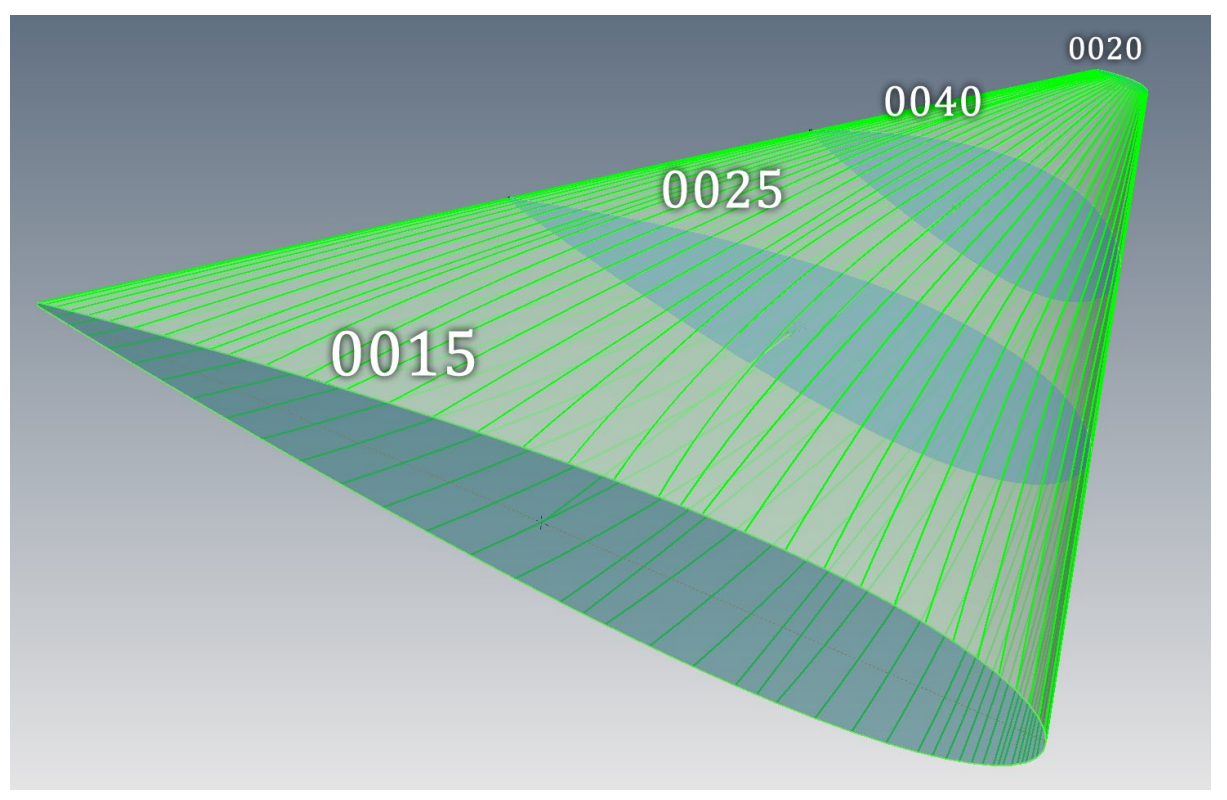

Figure 4.17: Fin shape based on beam length and minimum thickness. Numbers indicate the four-digit NACA code for the foil section shown. The final shape was created by lofting between these foil sections.

dimensional amplitude, $A / b$, achieved by the rigid fin rotating through angle $\theta$ is given simply by $\sin (\theta)=A / b$. A typical batoid amplitude of $A / b=0.5$ gives $\theta_{\max }=30^{\circ}$. Using values of $c_{d}=1.98$ and $\rho=1000 \mathrm{~kg} / \mathrm{m}^{3}$ (and values for $b$ and $c_{\text {root }}$ given above), the maximum force over the entire fin is estimated to be $F_{\text {plate }}=4.2 \mathrm{~N}$. This gives a final distribution at each node of $P_{j}=[0.50,1.50,2.17] \mathrm{N}$. Using these values of $P_{j}$, the change in tension of each cable due to this loading distribution is determined by equation 4.22 , giving $\Delta T_{j}=[16.2,12.1,4.5] \mathrm{N}$.

The torque required to resist all of these cable loads depends on the radii $r_{j}$. These are determined by $r_{j}=j r_{0}$ for an $\mathrm{MC}$ beam and using equation 4.13 to determine an appropriate value of $\bar{r}_{0}$. For a desired amplitude of $\bar{A}=0.5$, the aspect ratio $L / h=12.4$ (determined by beam parameters above), and an example $\theta_{\max }=30^{\circ}$, this gives a nominal radius of $\bar{r}_{0}=0.077$. However, this gives a drum radius corresponding to the first cell of $r_{0}=\bar{r}_{0} L_{0}=0.128 "$, which is quite small, making manufacturing difficult. As mentioned in section 4.1, the radius given by equation 4.13 is merely a guideline and not a strict design requirement. In order to improve manufacturability, 
the size of the radius is increased to $r_{0}=0.3$ ", corresponding to $\bar{r}_{0}=0.18$. To achieve the same amplitude of $\bar{A}=0.5$ with this radius, the necessary drum rotation is reduced to only $\theta=12.8^{\circ}$. The numerical procedure outlined in section 4.1 is used for $\bar{r}_{0}=0.18$ to find the optimal cam shape parameter $\bar{m}=0.0336$. Putting the results in real units, the radii corresponding to each cell of the tensegrity are $r_{j}=[0.3,0.6,0.9] "$ and the cam shape parameters for each portion of the drum are $m_{j}=[0.056,0.112,0.168]^{\prime}$.

Using these drum radii and cable loads, the total maximum torque expected on the actuation drum during flapping ${ }^{12}$ is $\tau_{\text {tot }}=0.41 \mathrm{Nm}$, given by equation 4.23 . This estimate of torque allows for the selection of an appropriate rotary actuator to drive the tensegrity beam. Ultimately, a servomotor was chosen actuation due to ease of position control and compact size. The model HS-7950TH by Hitec has a maximum torque of $3.4 \mathrm{Nm}$, which is more than sufficient given the estimate above ${ }^{13}$ It is rated for a maximum (no load) rotational speed of 462 degrees/s, and a maximum rotational range of $151^{\circ}$ was measured. Although it is expected that the angular position (and thus angular velocity) of the servo will align closely with the position commands given to the controller, the servomotor was modified so that analog voltage signals from the servo's internal potentiometer could be measured during all tests in order to verify the angular position.

With design parameters set and an actuator chosen, the actuation system can be fabricated and assembled. The final cam shape design is modeled and fabricated using a fused deposition modeling 3D printer. The material used is acrylonitrile butadiene styrene (ABS) polymer with $0.01 "(0.254 \mathrm{~mm})$ resolution. The servomotor drives the cam drum using a $1 / 4 " \varnothing(6.35 \mathrm{~mm})$ aluminum shaft, with a torque sensor mounted between the two in order to measure power input for the hydrodynamics

\footnotetext{
${ }^{12}$ Due solely to fluid loading from dynamic pressure. This torque estimate does not account for the acceleration of the fin or surrounding water.

${ }^{13}$ Having such a high factor of safety (more than 8 ) for the maximum torque is desirable, because the servomotor's speed is load dependent — as the load on the servomotor approaches its maximum, speed can drop considerably.
} 


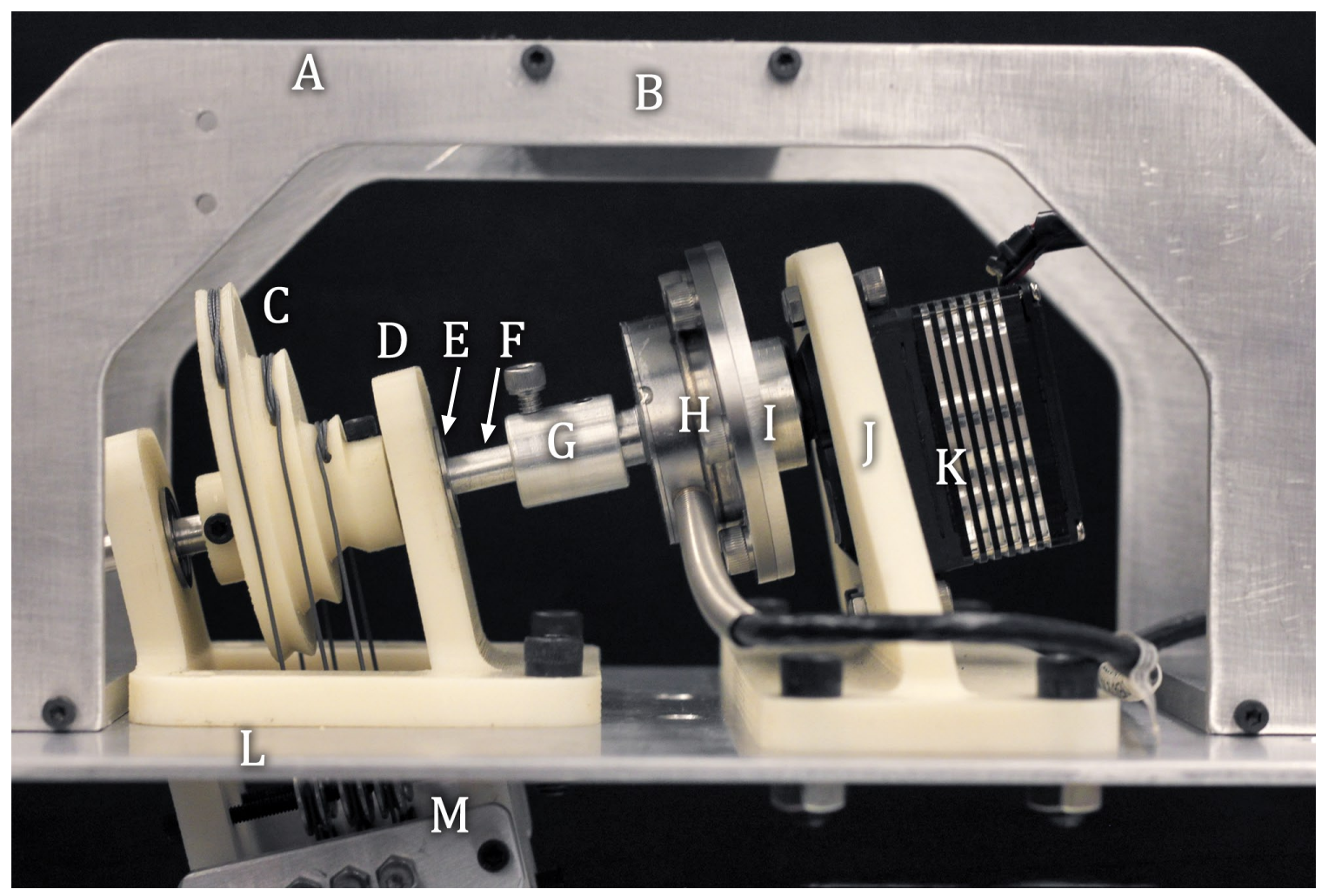

Figure 4.18: Actuation system for the active tensegrity beam. The cam drum (C) is mounted on an aluminum shaft $(\mathrm{F})$, which connects to the torque sensor $(\mathrm{H})$ with coupler $(\mathrm{G})$. The sensor connects to the servomotor $(\mathrm{K})$ with a flanged hub (I). The shaft rotates on ball bearings (E) placed in the cam/tensegrity mount (D). Both this mount and the servo mount $(\mathrm{J})$ are attached to the main aluminum chassis $(\mathrm{L})$. The cam/tensegrity mount penetrates through the chassis, leading to the tensioning system (M) and tensegrity beam below. The frame structure (A) allows the whole assembly to attach to the water tunnel rail from above at $(B)$.

experiments (explained further in section 5.1). These actuation system components are mounted to a common aluminum chassis, again using ABS printed parts. The assembled system is shown in figure 4.18 .

The struts of the tensegrity beam are machined with a CNC mill from 1/8" (3.18 $\mathrm{mm}$ ) thick aluminum sheets and the flanged ball bearings are pressed into the ends of each strut to create the nodes of the tensegrity structure. The vertical struts have holes at each end to permit the actuation cables to either route through to the 


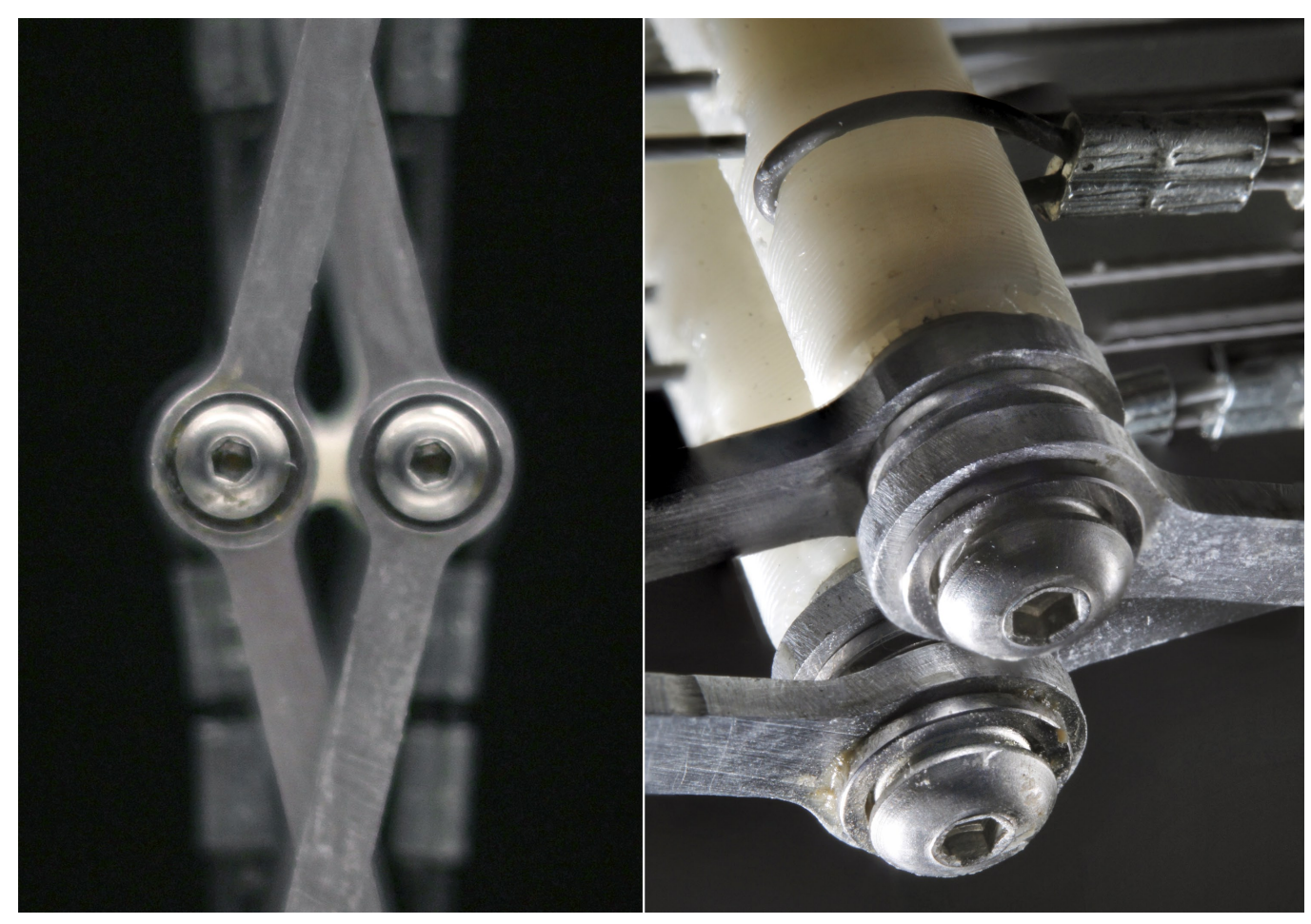

Figure 4.19: Detail of assembled nodes, showing aluminum struts connecting to a vertical strut (ABS plastic) with button head screws going through the ball bearings. Also in the perspective view, a cable from the first cell terminates with a cable crimp, while the two other cables route through the node.

next cell or terminate ${ }^{14}$, and they are 3D printed from ABS plastic. The terminal vertical strut has a 1.5" $(3.81 \mathrm{~cm})$ long extension in the span-wise direction in order to stiffen the tip of the fin. The aluminum struts attach to each vertical strut using 5-40 button-head machine screws through each bearing. Joint assembly details are shown in figure 4.19 .

The assembled tensegrity beam is mounted on the chassis in line with the cam actuation drum. Nylon-coated braided stainless steel cables $0.024 " \varnothing(0.61 \mathrm{~mm})$ rated at $70 \mathrm{lbf}(311 \mathrm{~N})$ breaking strength are routed through the structure. Each cable pair is actually one continuous cable that is threaded through the cam drum such that it

\footnotetext{
${ }^{14}$ Ball bearing pulleys were omitted from this structure in the interest of minimizing beam thickness. It was found that routing nylon-coated braided cables through filleted holes in the ABS resulted in a node with acceptably low friction.
} 
does not slip 15 , and each end of the cable is terminated in the structure with copper crimps (also shown in figure 4.19). The cables are attached to the beam while in a vertical hanging orientation to give them the correct nominal lengths.

In order to adjust the pretension of the cables in the structure, a tensioning system was devised, consisting of a pulley attached to a lead screw and two idler pulleys (see figure 4.20. As the lead screw is driven inwards, the cable deflects between the two idler pulleys. Since the cable is fixed at both ends (one end terminates in the structure and the other end at the actuation drum), the tension in that cable increases by the action of the lead screw. There are six of these tensioning mechanisms in total, corresponding to both the top and bottom cables of all three cells of the beam.

Using the tensioning mechanism, the pretension is increased slightly in each cable to achieve a stable prestress state for the structure while the neutral position of the beam is maintained. Then the correct pretension is set by considering a conservative estimate for the critical slackening load: if the entire load $F_{\text {plate }}$ is applied at the tip and no cables go slack, then the structure is considered properly tensioned. To accomplish this, $F_{\text {plate }}$ is applied to the beam tip as hanging brass weights and the tension in each cable is recursively increased (on the side of the beam corresponding to decreasing tension from the tip load) until this no-slack condition is met. The process is repeated for the other side of the beam, and then checked once again for each side of the beam. Unlike the larger static tensegrity beam from section 3.2 , there is no room for in-line load cells in this compact structure, so the condition of slackness is determined manually. While this method is somewhat subjective and exact tension values are unknown $[16$, the transition from slackness (no tension) to some tension is quite distinct, so the method at least ensures the beam will not experience critical slackening under this load. The assembled, pretensioned beam attached to the test

\footnotetext{
${ }^{15}$ The cam drum is designed with holes that tunnel through each radius so that the cables loop through several times, essentially forming a self-tightening knot around part of the drum. This configuration was never found to slip by any measurable amount for any external load applied.

${ }^{16}$ Estimating the error in this tension is difficult, but based on extensive prototyping with other tensegrity beams, it is likely on the order of $5 \mathrm{~N}$.
} 


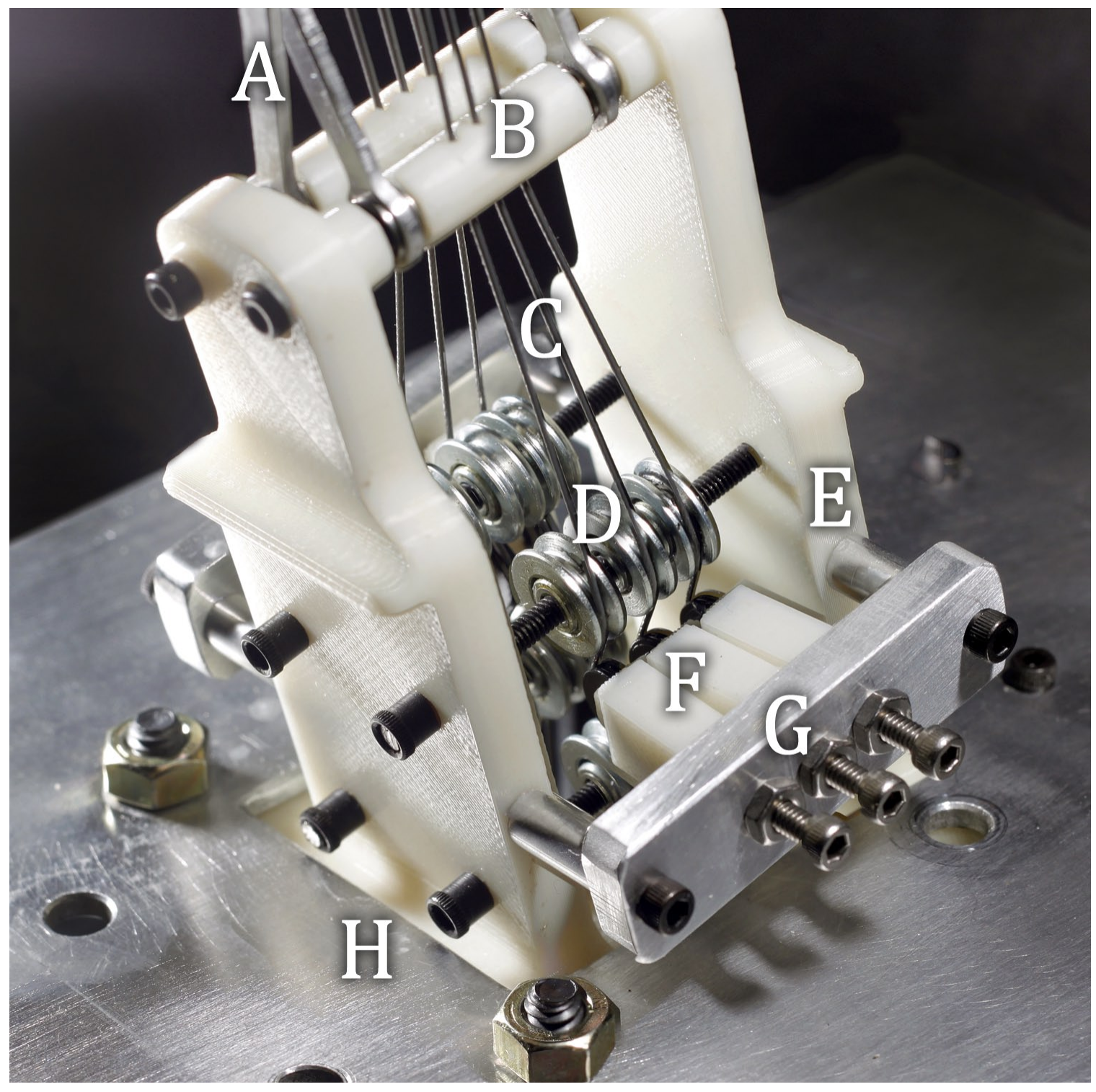

Figure 4.20: Detail of tensioning mechanism in the active tensegrity beam. Lead screws $(\mathrm{G})$ drive tensioning arms $(\mathrm{F})$, made of acetyl pulleys allowed to freely rotate in a lubricated pocket. These tensioning arms deflect the cables between sets of idler pulleys (D) before the cables $(\mathrm{C})$ route through the base nodes (B) of the tensegrity structure (A). The mechanism is rigidly attached to the tensegrity mount (E), which penetrates through the main chassis $(\mathrm{H})$. 

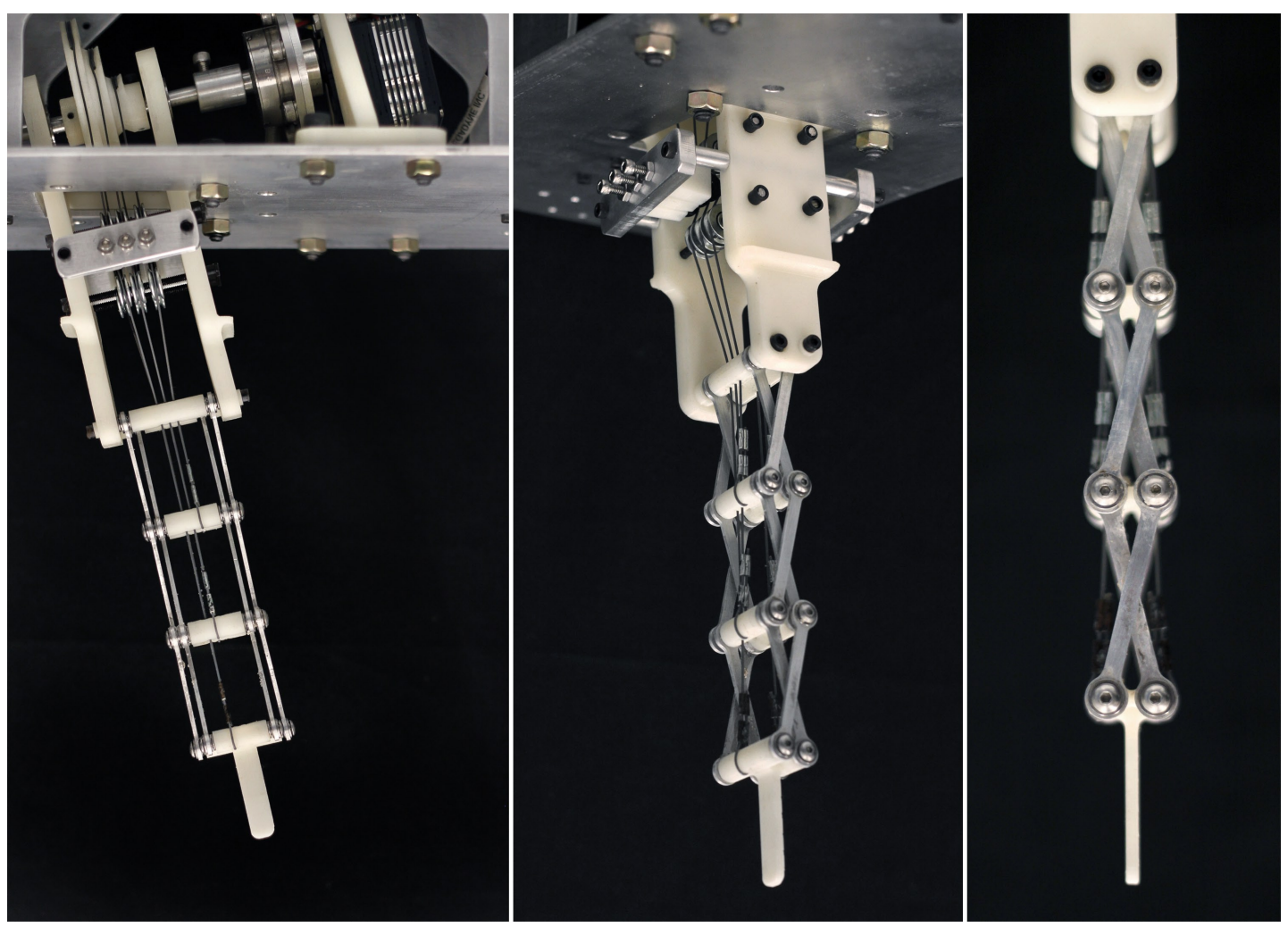

Figure 4.21: The active tensegrity beam assembled and pretensioned, shown in three different views. The extension on the terminal node stiffens the tip of the fin.

chassis is shown in figure 4.21 .

To turn the fin shape design (figure 4.17) into a mold for making the skin, the 3D model is inverted so that the fin shape is a cavity within a solid block that is then split in two. The root chord section was extended 1" $(2.54 \mathrm{~cm})$ in order to allow for embedding anchors that hold the skin in place (these anchors were 3D printed using ABS and attach to the main chassis). This model was used to fabricate a two piece mold from $1.5 "(3.81 \mathrm{~cm})$ thick acrylic sheets using a CNC mill, shown in figure 4.22 .

Using this mold, a skin material can be set around the tensegrity beam to form the final fin shape. The material used to make the skin is a two-part addition curing (platinum catalyst) liquid silicone rubber (which sets at room temperature) called Ecoflex from Smooth-On, Inc. The formula used is rated at a nominal Shore hardness of OO-10, but a non-reactive silicone thinner was added to comprise $20 \%$ of the mixture (by weight) in order to make the final material even softer and more flex- 


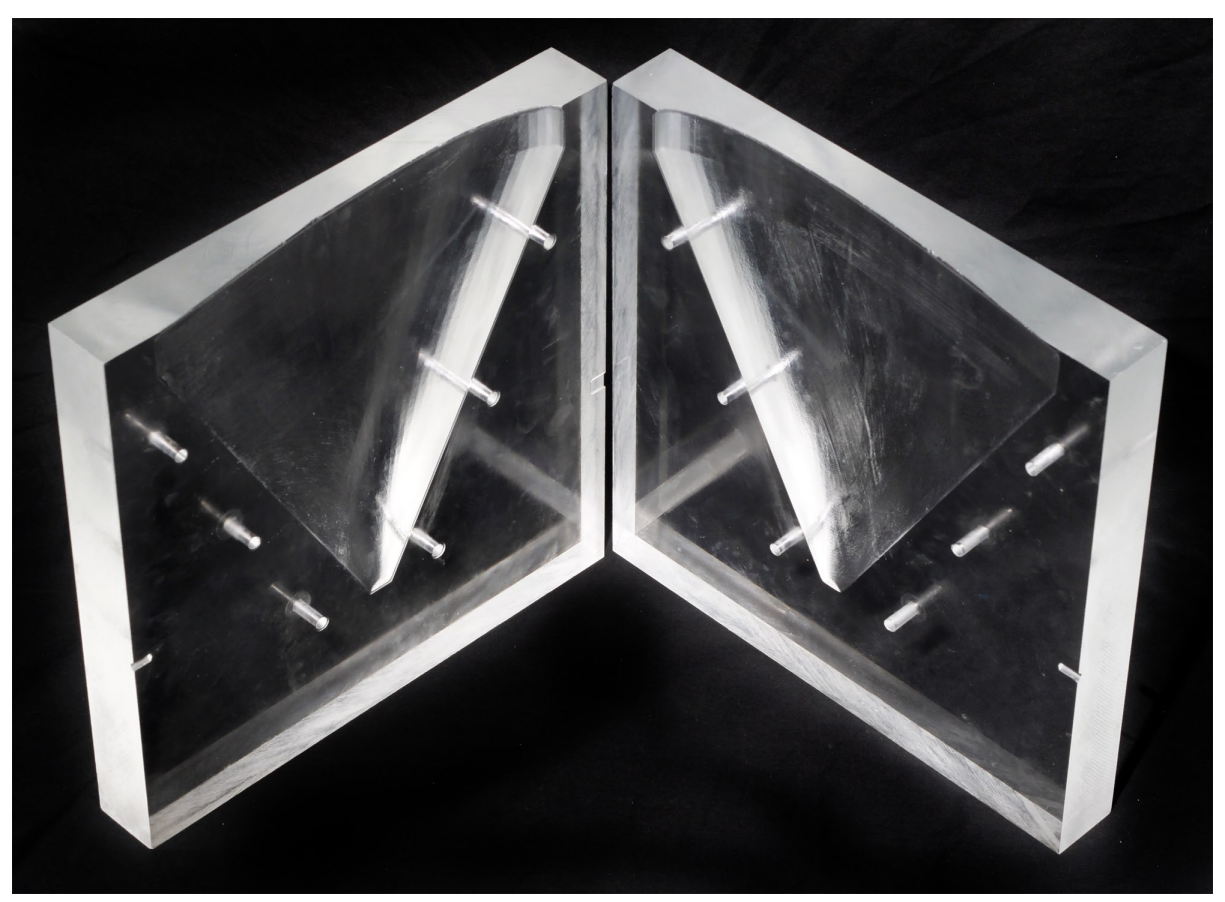

Figure 4.22: Mold for skin, based on fin shape from 4.17 and milled from acrylic.

ible. This material is slightly positively buoyant when cured. The tensegrity beam was encased in a close-fitting polyisoprene sleeve in order to exclude the liquid skin material from the beam while molding. The entire fin assembly was placed on top of the closed mold using mounting guides to ensure proper positioning and alignment. The two-part liquid silicone (plus thinner) was mixed, degassed, and poured into the mold. When fully cured, the mold was separated and the fin was released. At this point the skin is removable, by detaching the skin anchors from the chassis and simply sliding the skin off of the beam. In this way, inspections, repairs, or adjustments can easily be made to the underlying beam and the skin is interchangeable so that different skins can be tested. The entire assembly with the skin attached is shown in figure 4.23 . 


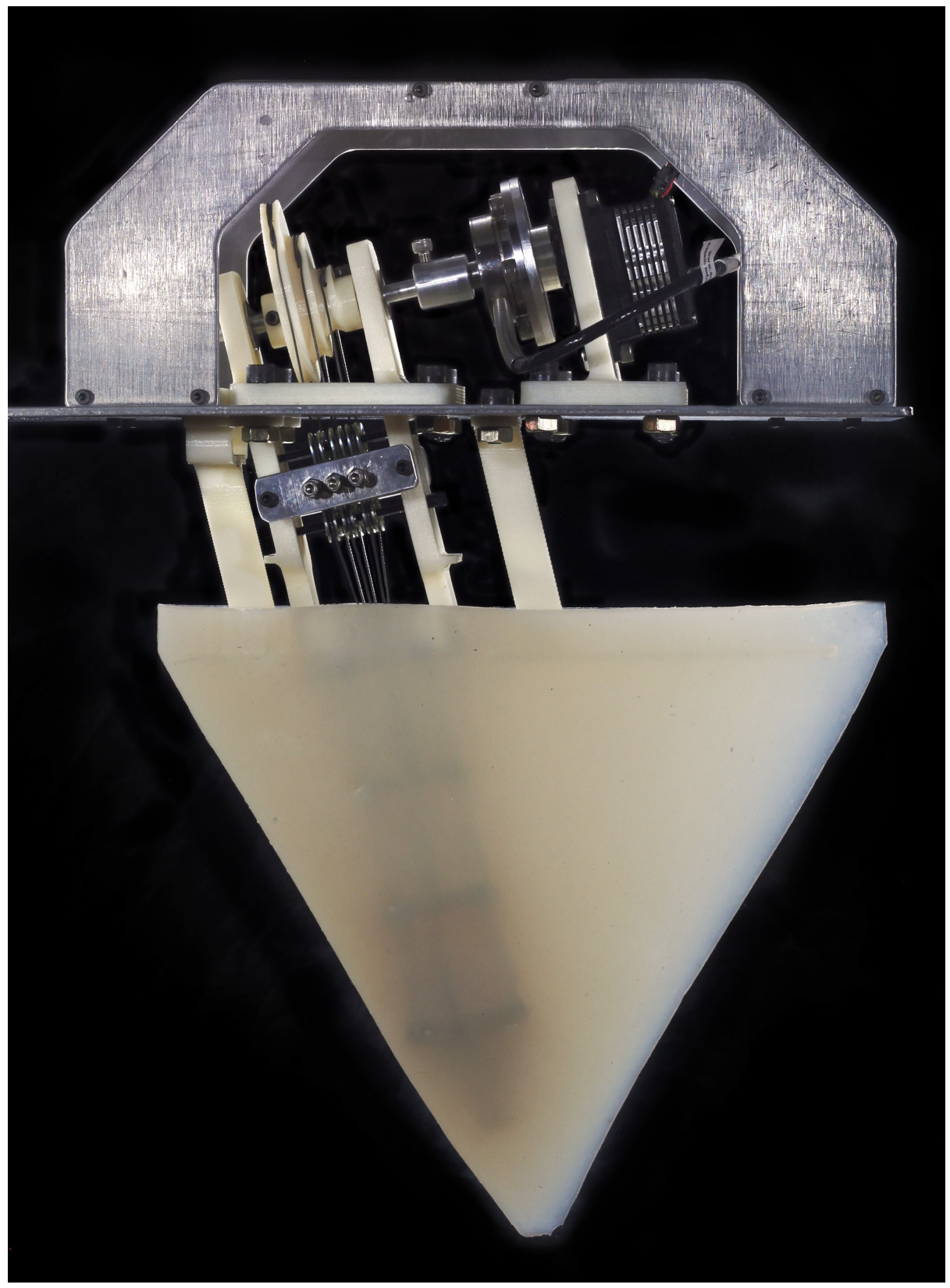

Figure 4.23: Complete fin, with tensegrity embedded in elastomer skin. 


\subsection{Actuation Results}

Using the fully-assembled tensegrity beam described in the previous section, the active bending amplitude can be measured and compared to the linearized analytical prediction for bending amplitude, which is obtained for an MC beam by rearranging equation 3.1 .

$$
A \approx \frac{\delta_{\mathrm{a}, j} N L}{h j}=\frac{r_{0} \theta N L}{h}
$$

Where the actuation amount $\delta_{\mathrm{a}, j}$ for the $\mathrm{MC}$ beam can be approximated by a circular actuation drum, as discussed in section 4.1. In order to predict bending amplitudes for large input actuation (which is expected to deviate from the linearized prediction), a full, non-linearized analytical prediction for the 3-cell beam has been adapted from [4] to give:

$$
A=\frac{L}{N}\left(\sin \frac{r_{0} \theta}{h}+\sin \frac{3 r_{0} \theta}{h}+\sin \frac{5 r_{0} \theta}{h}\right)
$$

Additionally, with an artificial skin in place, the tip amplitude of the whole fin (which is the measure of interest for hydrodynamic purposes) can be predicted and tested as well. The total fin amplitude can be estimated by assuming that the tip stiffener (see figure 4.21) and tip portion of the molded silicone skin beyond the stiffener undergo a rigid body rotation, the angle of which is determined by the rotation of the terminal face of the tensegrity beam. Using the geometry of the deformed tensegrity cells, this additional tip amplitude is given by:

$$
A_{\text {tip }}=L_{\text {tip }} \sin \left(2 N \arctan \frac{r_{0} \theta}{h}\right)
$$

To experimentally obtain the actuation amplitudes, tests are conducted measuring the amplitude of both the beam tip and fin tip over a range of rotational positions of the actuation drum. Positioning of the active tensegrity beam is prescribed by 
rotation of the servomotor, which connects to the cam-shaped actuation drum, which then in turn simultaneously contracts and releases cables to activate the bending mode of the beam.

For the purposes of both amplitude tests and for actuating the flapping fin in the hydrodynamics tests of chapter 5 , the servomotor is controlled by a LabVIEW program that outputs position signals to a Parallax USB Servo Controller board. This board then translates these position commands and sends them to the servomotor's internal controller in the form of a pulse-width modulated (PWM) signal. The theoretical positioning resolution of this control arrangement is $0.2^{\circ}$, corresponding to $\bar{\delta}_{\mathrm{a}}=6 \times 10^{-4}$ and $A=1 \mathrm{~mm}$ (based on the linear prediction).

For amplitude tests of the beam alone with no skin, the servomotor is statically set to rotational positions in the range of $\pm 30^{\circ}$, which corresponds to a maximum nondimensional actuation of approximately $\sqrt{17} \bar{\delta}_{\mathrm{a}}=0.095$. Amplitude measurements are made optically, by taking photos of the beam with a camera positioned orthogonal to the plane of beam movement, as shown in figure 4.24. Amplitude is measured as the perpendicular distance from the middle of the terminal vertical strut (as with the experiments of section 3.2 to the line of neutral position of the beam.

The measured amplitudes are plotted against rotational position in figure 4.25 . Both the linearized prediction of actuation amplitude (equation 4.24) and the exact analytical solution (equation 4.25) are overlaid for comparison. The results show the tensegrity beam is capable of achieving large amplitude bending, up to $A / L=0.73$ at $30^{\circ}$. Notice that the exact solution starts to noticeably diverge from the linear prediction at high amplitudes, above $A / L \approx 0.4$ (because the linear prediction is based on a small angle assumption that breaks down). The exact solution accounts for the fact that at a high enough level of actuation, the beam tip is displacing noticeably in the span-wise direction $(-x)$, and therefore the slope of the amplitudeactuation curve falls. This same trend is exhibited in the experimental results, which

\footnotetext{
${ }^{17}$ Using the antagonistic circular drum prediction; the actual contraction and release actuations will be less than and greater than, respectively, this estimate because of the cam shape.
} 


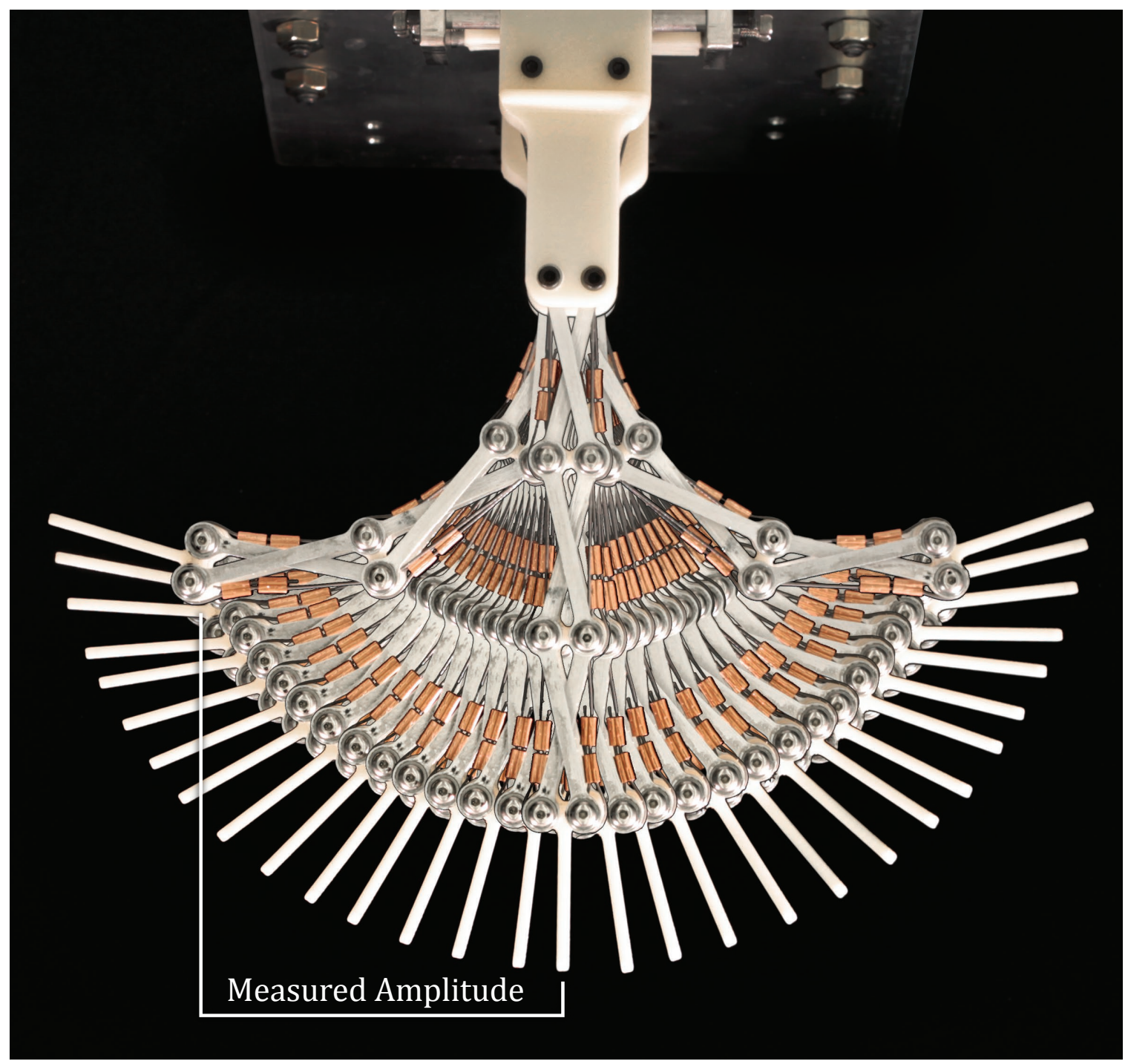

Figure 4.24: All images from the optical amplitude measurements are overlaid in composite to demonstrate the actuation range of the active tensegrity beam. Each image corresponds to servomotor rotation increments of $2^{\circ}$. The neutral and maximum amplitude positions are shown atop the intermediate amplitudes. Measured amplitudes are taken from the end of the terminal cell, not the end of the tip stiffener. 


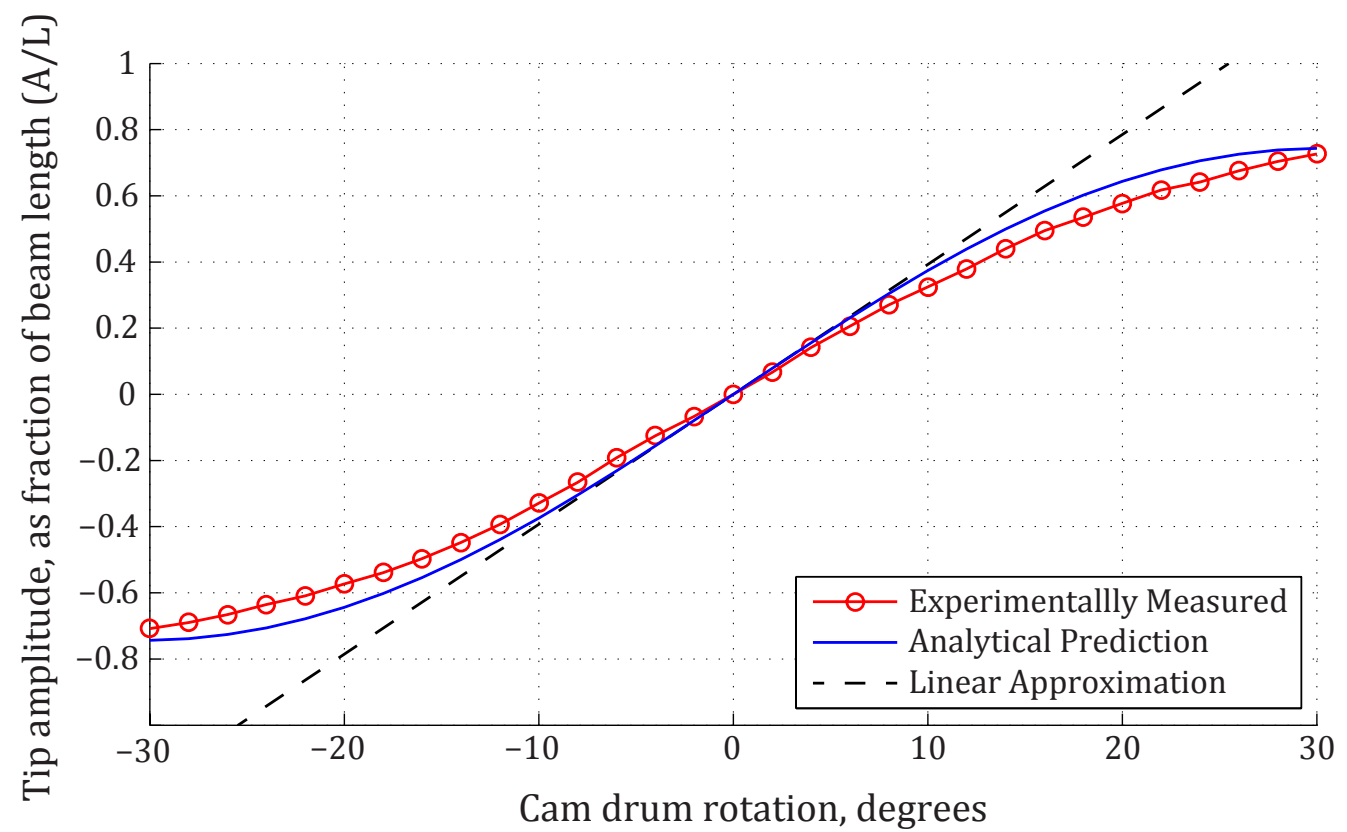

Figure 4.25: Plot of active tensegrity beam amplitude versus actuation drum rotation.

have a general shape quite close to the exact solution, however the actual amplitudes for some rotational positions $\left(8^{\circ}\right.$ to $\left.28^{\circ}\right)$ are somewhat lower than the exact solution, on average being about $10 \%$ lower.

While it is not completely clear what caused this deviation, there are a few likely sources. First, the beam was actuated with a cam drum, not perfectly antagonistically as in the analytical predictions; since the radius on the contraction side of the cam is slightly smaller than it would be for an equivalent circular drum, this would contribute to the decreased amplitude. Second, some components in the structure are made of ABS plastic, which is significantly softer than the rest of the components (that are either aluminum or stainless steel), so it is possible that some of these plastic components are deforming during actuation such that amplitudes are made slightly lower. Third, there is some friction experienced by all moving components in contact and the cables are not routed over low friction pulleys within the beam, but rather through small holes, so some amount of rubbing on the cables could be resisting the beam's actuation. 


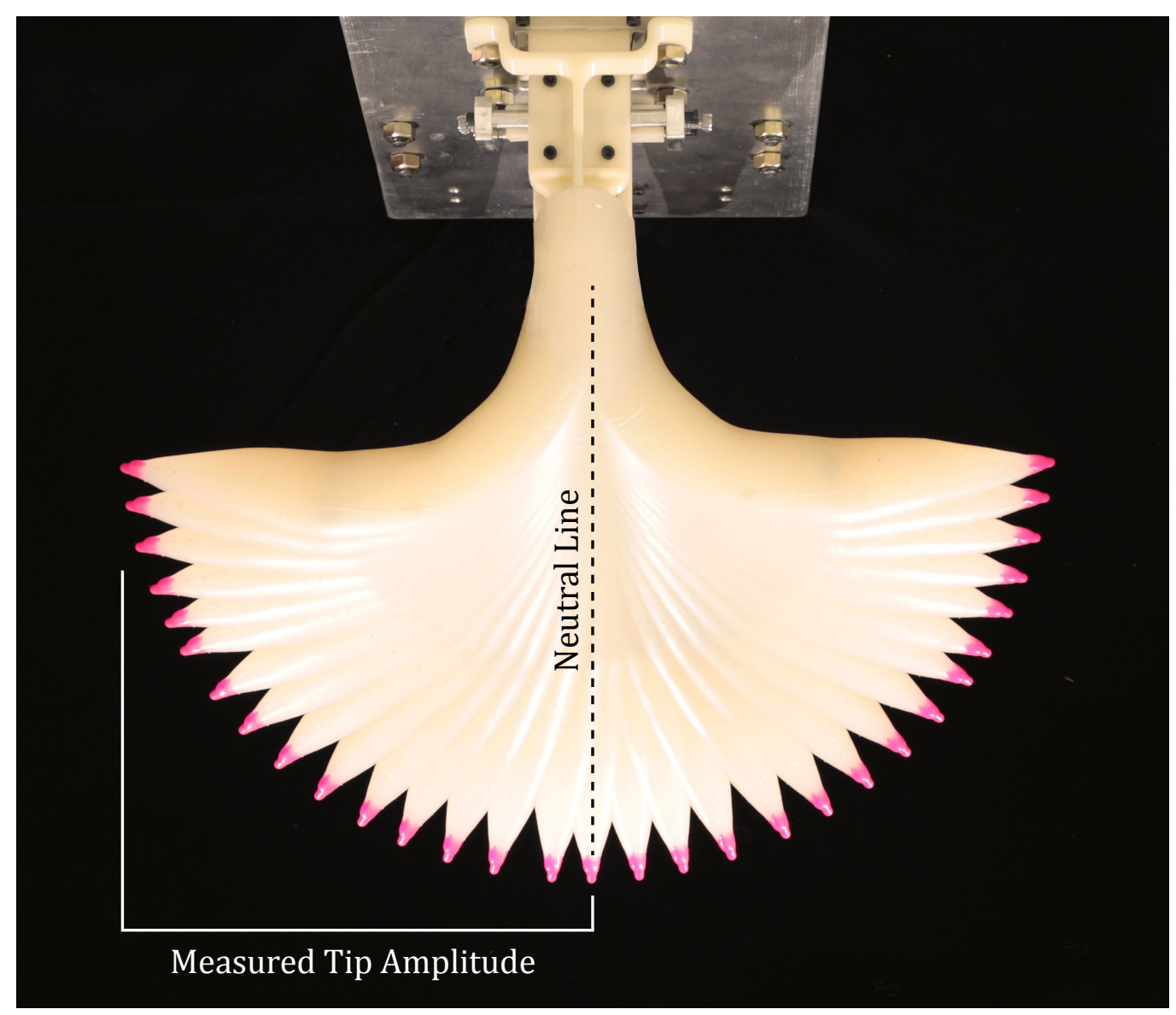

Figure 4.26: The fin is actuated in a horizontal plane and the amplitude from the neutral line at each position is measured optically.

Despite the fact that the bending amplitudes are slightly lower than predicted, these results quantify the relationship between drum rotation amount and unloaded tip amplitude for this beam, making it possible to achieve a desired nominal tip amplitude using this data. Additionally, for the purposes of hydrodynamic testing, the amplitude of the fin tip is the measure of importance, not the underlying beam, so fin tip amplitude is measured next.

The amplitude of the fin tip is measured in a manner similar to the tensegrity beam alone, except with the artificial skin on, as shown in figure 4.26. This shows the fin is clearly the fin is capable of large span-wise bending amplitudes.

Figure 4.27 shows the measured amplitudes normalized by fin span length and plotted against drum rotation angle. Measurements were made for drum rotation 


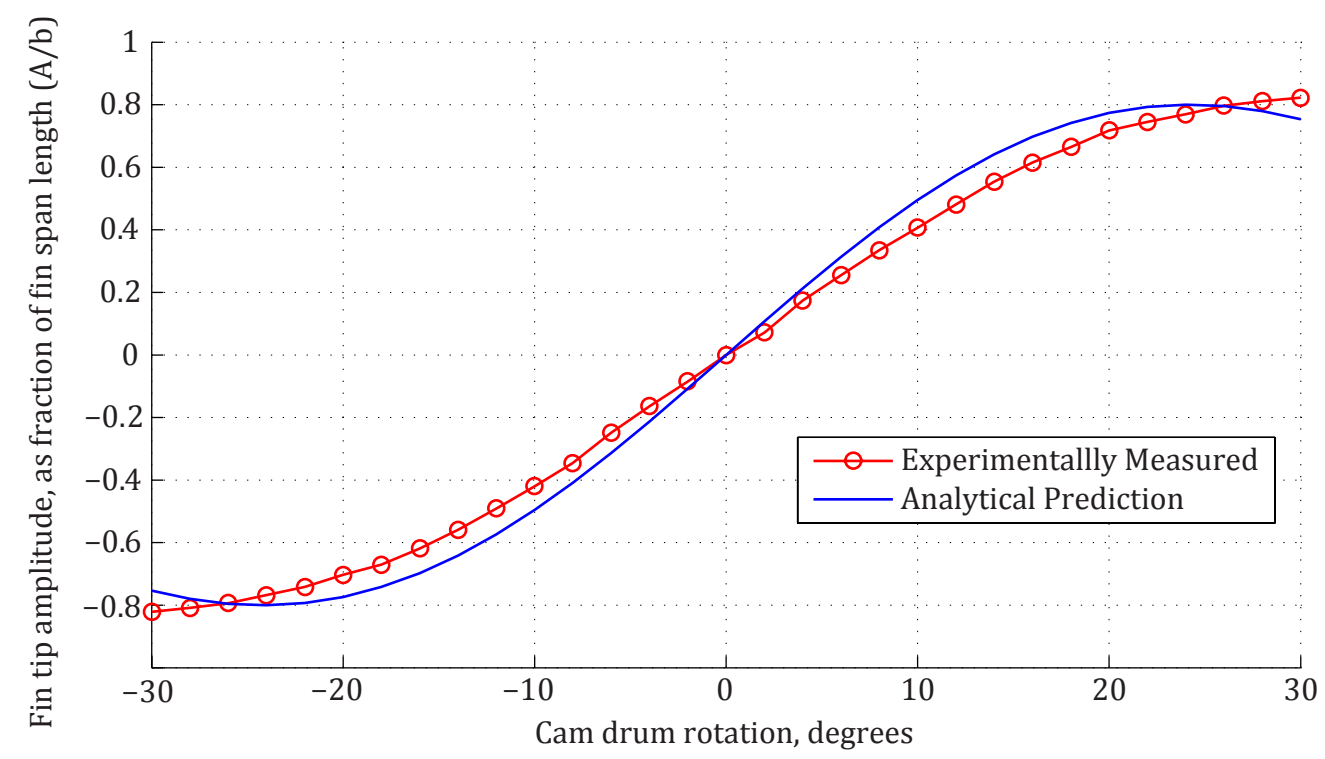

Figure 4.27: Plot of optically measured fin amplitudes versus actuation drum rotations.

angles in the range of $\pm 30^{\circ}$. The analytical prediction used for the tensegrity beam alone can be extended to apply to the whole fin by assuming the fin length beyond the end of the tensegrity beam (this includes the 1.5" stiffener attachment seen in figure 4.24 and another 1.5" of passive skin material) is a straight, rigid segment that rotates with the angle of the terminal face of the tensegrity beam. The measured amplitude matches fairly well to the analytical prediction, although as with the tensegrity beam the amplitude is slightly lower for most of the actuation range (also about $10 \%$ lower, averaged across all points), which should be expected if the underlying tensegrity amplitudes are lower than the analytical prediction.

Overall, these experimental results show that for low amplitudes (up to about $A / L \approx 0.4)$, the linear prediction of section 3.1 works reasonably well for predicting tensegrity beam tip amplitudes. For higher amplitudes, the linear prediction breaks down and experimental results track more closely with the exact analytical solution, although a large portion of the measured amplitudes are around 10\% lower than expected. Measurements of the entire fin — the tensegrity beam with the artificial skin on - demonstrate that large amplitude flapping (up to $A / b=0.82$ ) can be 
achieved with this system. These results only reflect amplitudes with no external loading and it is expected that amplitudes will be lower when hydrodynamic loading is applied to the fin. This scenario is investigated in the next chapter, where the fin is subjected to hydrodynamic testing and amplitudes in the water tunnel are again measured optically.

In summary, this chapter has demonstrated several important contributions towards the overall goal of being able to quantify swimming performance for an artificial pectoral fin. It sets forth the concept, design, and fabrication of an artificial fin that meets the basic requirements and constraints of the hydrodynamic experiments. In support of this effort, the amount of modified antagonistic actuation necessary for minimizing induced strains was quantified, which directly enabled the design methodology laid out for a cam-shaped actuation drum. Additionally, an estimate was given for fluid loading on the fin during flapping, which allowed for the sizing of components and proper pretensioning of the tensegrity beam. Lastly, bending amplitudes relative to input actuation were quantified and the fin demonstrated the desired span-wise bending mode, similar to the kinematics observed in biological oscillatory rays. All of these individual components are necessary for the creation of the fin which is used in the following chapter to quantify swimming performance. 


\section{Chapter 5}

\section{Hydrodynamic Experiments}

With the ability to reproduce the major kinematic motions seen in batoid rays using the artificial batoid fin described in the previous chapter, the goal is to quantify the swimming performance of this robotic fin by taking specific bulk measurements in hydrodynamic experiments while systematically varying kinematic parameters. Of greatest interest is the relationship between thrust production and two important flapping parameters: frequency (temporal) and amplitude (spatial). Swimming performance is measured in a water tunnel with two different types of tests, in which the fin is either constrained or unconstrained to move along the direction of flow. Additionally, the effects of several other experimental variables are explored: the existence of a free surface in the water tunnel, the imposed flow speed in constrained tests, allowing for acceleration in free swimming, and stiffness of the fin's artificial skin material. What follows in this chapter is a description of both the experimental methods and the results of hydrodynamic tests performed with the tensegrity-based fin. 


\section{$5.1 \quad$ Experimental Setup}

As introduced in chapter 2, a variety of methods for quantifying swimming performance (both biological and artificial) have been established by others [63, 65, 125, 126]. One of the prevailing measures for the performance of flapping propulsors is Froude propulsive efficiency, which is a non-dimensional ratio of output power to input power. Determining Froude propulsive efficiency requires the measurement of net thrust produced at a known flow speed, and also a measurement of mechanical power input to the fluid. In addition to this efficiency measure, free-swimming performance of the fin, which can be measured as swimming economy, is also of interest. The experimental rig should therefore be capable of two types of tests: constrained, in which the relative flow speed across the fin is fixed, for measuring thrust and efficiency; and unconstrained, in which the fin is allowed to freely swim in the stream-wise direction, for measuring free-swimming speed and economy. The following is a description of the experimental test rig designed and constructed to quantify the net thrust produced by the fin and the free-swimming speed of the fin for these two types of tests.

\subsubsection{Equipment}

All experiments related to swimming performance were carried out using a recirculating water tunnel (also known as a "flow tank") in which the fin can flap submerged up to the root chord line. The equipment used is a Model 1520 water tunnel made by Rolling Hills Research Corporation. The test section of this water tunnel has a width of $15 "(38.1 \mathrm{~cm})$, depth of $20 "(50.8 \mathrm{~cm})$ and length of $60 "(152.4 \mathrm{~cm})$. The construction of the tunnel allows for viewing from three orthogonal directions. The maximum flow speed is $36 \mathrm{in} / \mathrm{s}$ (approximately $0.9 \mathrm{~m} / \mathrm{s}$ ) with turbulence of less than $1 \%$ RMS. Flow velocity can be set with a resolution of $0.1 \mathrm{in} / \mathrm{s}(2.5 \mathrm{~mm} / \mathrm{s})$ and is controlled by a calibrated flow sensor embedded in the return side of the closed-loop system. It was found that in practice, the flow control system resulted in a reading 
of flow speed that could fluctuate randomly within $\pm 0.1 \mathrm{in} / \mathrm{s}$ of the set speed, so it is assumed that the standard deviation in flow speed measurements is $0.058 \mathrm{in} / \mathrm{s}$ $(1.5 \mathrm{~mm} / \mathrm{s})$.

To allow for free swimming along the length of the test section (along the direction of flow), the fin (described in section 4.4) is mounted to a slider bearing on a linear motion guide rail. This setup also isolates the forces from the fin to act only along the stream-wise direction for the thrust measurements of the constrained tests. The linear rail hardware is a model LWLF18-BCS from IKO, which was selected because it has the smallest form factor that comes in the length desired for the water tunnel test section ${ }^{1}$. The load rating for this model is $2.3 \mathrm{kN}$, which is more than sufficient for the weight of the fin assembly, which is $22 \mathrm{~N}$ (out of water). The rail is $1.3 \mathrm{~m}$ long, which provides more than 5 full chord-lengths of travel for the fin.

The static friction along the rail was estimated by placing a brass weight on the slider bearing and slowly raising one end of the rail until the slider bearing traveled freely. By simple trigonometry the component of the weight acting along the rail direction is determined by the rail length and elevation of the raised end of the rail. After some modifications to the linear motion system (sandblasting material from the rail channels, removing the slider bearing end seals, and replacing the stock lubricant with a far less viscous one), the maximum resistance to sliding was found to be less than $0.1 \mathrm{~N}$.

The linear motion guide rail is attached to a rigid mounting frame for the entire test rig. The frame is constructed from aluminum U-channels and is designed to straddle the rails of the water tunnel with adjustable length feet such that the fin can be submerged in the free surface of the water tunnel just up to the root chord line. A $1 / 4$ " thick acrylic plate can be attached to the frame with threaded rods so that the height of the plate can be adjusted to meet the free surface of the water in

\footnotetext{
${ }^{1}$ The sliding resistance of these systems generally scales with size, so the smallest form factor was desired in order to have low friction. However, maximum monolithic rail length also scales with form factor, so a very small (low friction) form factor rail would also be very short, requiring abutted joints, which are difficult to align properly.
} 


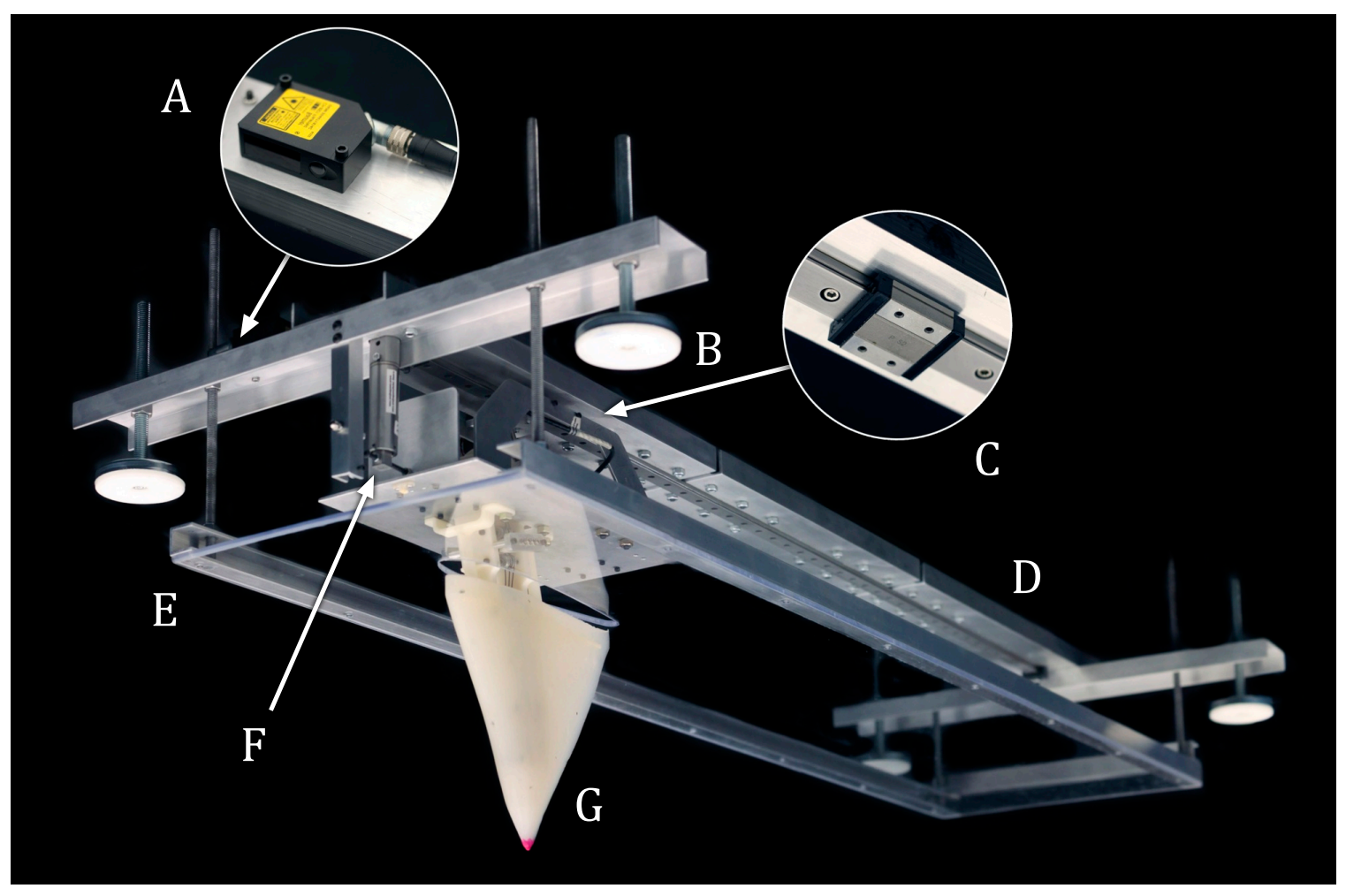

Figure 5.1: Assembled test rig for flow tank experiments. The tensegrity fin $(\mathrm{G})$ is attached to the linear motion guide rail with a slider bearing $(\mathrm{C})$. The rail is attached to the mounting frame (D), which straddles the side rails of the water tunnel with adjustable feet (B). In constrained tests, thrust measurements are taken with the load cell $(\mathrm{F})$ and in unconstrained tests, position along the rail is measured with the laser distance sensor (A). The height of the surface wave suppression plate (E) can be adjusted to meet the fluid surface in the water tunnel.

the tunnel. This plate has a hole cut out that is slightly larger than the cross section of the fin at the root chord, so that the fin can penetrate through the hole and flap without contacting the plate. The purpose of this plate is to optionally suppress motions of the fluid's free surface, in order to evaluate the effect of waves and other surface disturbances. The assembled test rig is shown in figure 5.1.

To measure net thrust in the constrained tests, a strain gauge-based load cell is attached at the front end of the test rig's frame. The load cell used is an Omega model LC601-1, which has a range of $\pm 1 \mathrm{lb}( \pm 4.45 \mathrm{~N})$ with a stated repeatability of $0.02 \%$ of full-scale output $(0.9 \mathrm{mN})$. The fin carriage transmits force to the load cell via contact between a plate on the fin carriage and an acorn nut on a screw attached 


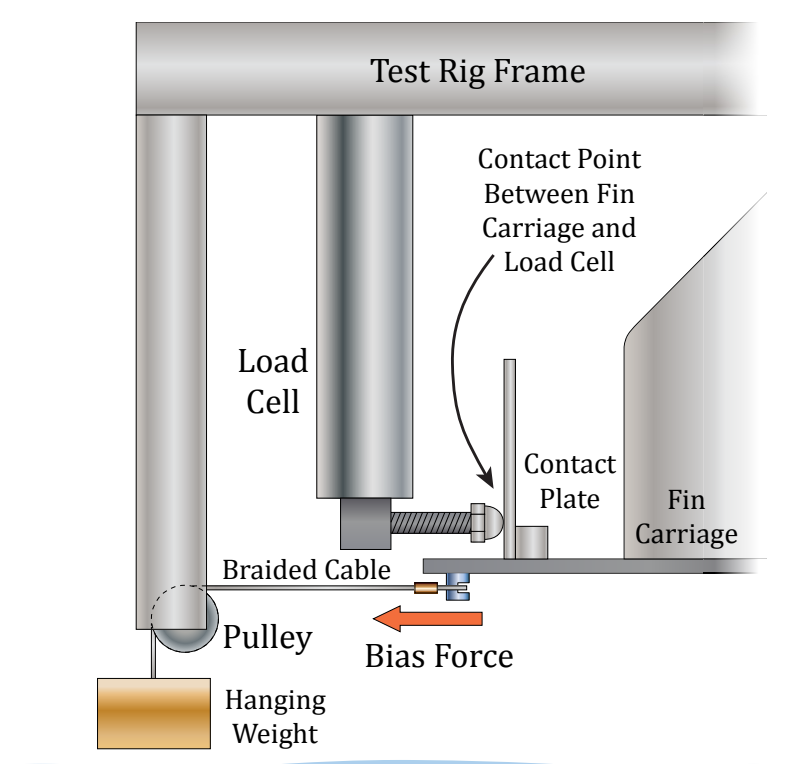

Flow Direction

Figure 5.2: Configuration for thrust measurements. As the fin produces net thrust, the force is transmitted through the fin carriage and onto an acorn nut attached to the load cell. The fin is biased forward by a hanging weight so that it remains in contact with the load cell even if there is drag.

to the load cell (see figure 5.2). The load is biased forward by hanging a $100 \mathrm{~g}$ weight over a low friction pulley. In this way, the fin carriage remains in contact with the load cell even if there is net drag (up to $100 \mathrm{gf}$ ) on the fin, and therefore this setup allows for the measurement of instantaneous net drag. This configuration with a single point of free contact also eliminates the possibility of binding, which could occur if the fin were rigidly attached to the load cell. The forward bias is simply zeroed out in the data recording system, so that net thrust/drag is measured relative to this biased configuration (with the flow off).

Free-swimming velocity in the unconstrained tests is measured using a laser distance sensor, which is mounted at the front of the frame such that it is aimed at a target on the fin carriage. By measuring the position of the fin along the rail during tests (without contact), the free-swimming velocity can be calculated by simply adding the fin's velocity relative to the water tunnel to the flow speed of the water. The laser distance sensor used is a Baumer model OADM 250U1101/S14C, which has 
a measurement range of $0.2-4.0 \mathrm{~m}$, resolution of $1.3 \mathrm{~mm}$, and stated repeatability of $\pm 5 \mathrm{~mm}$. The sensor works by triangulation, detecting the perceived lateral movement of the diffuse reflection of the emitted laser beam, so the target on the fin carriage is painted matte black.

To measure the mechanical power input to the tensegrity fin, a torque sensor is placed in the drive train of the actuation system between the servo motor and cam drum (as mentioned in section 4.4). The torque sensor used is an Omega model TQ202-25, which can measure \pm 25 in $\mathrm{lb}( \pm 2.8 \mathrm{Nm})$ of torque with a stated accuracy of $0.2 \%$ of full-scale output $\left(5.7 \times 10^{-3} \mathrm{Nm}\right)$.

The analog voltage signals from the torque sensor, servomotor internal potentiometer, load cell, and laser distance sensor are measured using a National Instruments USB-6259 data acquisition device and recorded with LabVIEW software at a sampling rate of $100 \mathrm{~S} / \mathrm{s}$. LabVIEW is also used to control the servo motor driving the tensegrity fin actuation (as described in section 4.5). The signals to and from the fin carriage (torque sensor, servomotor potentiometer, power and control to the servomotor) are carried over ultra-flexible cables (braided 26 AWG with silicone insulation) suspended from overhead with ample slack to ensure the cables do not apply any measurable amount of force to the carriage, so that thrust and speed measurements are unaffected.

\subsubsection{Experimental Procedures}

For the constrained efficiency tests, the flapping amplitude is set at a fixed nominal value that is typical for batoid rays and the flapping frequency is varied ${ }^{2}$ over a range similar to that seen in biology. A fixed flow speed is chosen to span a Strouhal number range which is expected to correlate to peak efficiency — as discussed in section 2.2, this range is generally considered to be $0.2<S t<0.4$. For example, a

\footnotetext{
${ }^{2}$ While it has been observed that oscillatory rays do not primarily use frequency to modulate thrust production [58, sweeping through a frequency range for this type of test is somewhat standard [4, 41, 65] and provides results that can be compared against literature.
} 
fixed amplitude of $A=10.2 \mathrm{~cm}$ (corresponding to $A / b=0.5$ ) and a frequency range of $0.5<f<1.25 \mathrm{~Hz}$, would produce a Strouhal number range of $0.2<\mathrm{St}<0.5$ for a flow speed of $U=0.25 \mathrm{~m} / \mathrm{s}$. Tests can then be performed with various combinations of flow speed or amplitude over a range of flapping frequencies. All flapping tests use a symmetric sinusoidal actuation waveform.

The constrained test protocol is as follows. The fin carriage is positioned at the front of the linear motion guide rail, attached to the forward bias weight with a braided cable so that the carriage is in contact with the load cell. With the water tunnel flow turned off and the fin motionless, the load cell measurement is balanced (zeroed). The flow velocity of the water tunnel is then set and turned on. Each test for a group of parameters (fixed $A$ and $U$, and a range of $f$ ) is then run for 12 flapping cycles ${ }^{3}$ while the resulting thrust production and power input are measured. Once the frequency range has been spanned to complete a set, each test set is then repeated 5 times so that mean values and standard deviations can be calculated. During the tests, the flapping fin is recorded with video and still images from both the spanwise and posterior (downstream) views, so that the in-water tip amplitudes can be measured and used in Strouhal number calculations and so that passive deformation of the skin can be observed. This procedure can be carried out either with or without the surface wave suppression plate.

The unconstrained free-swimming tests are similar to the constrained tests in that the actuation amplitude is held constant over a range of frequencies. However the procedure for the unconstrained tests differs in that the water tunnel flow speed is adjusted for each flapping frequency so that the fin travels upstream at a constant velocity for a minimum number of cycles over the length of the rail. This adjustment of flow speed ensures that the fin is indeed freely swimming and that there is an adequate number of cycles over which the data can be averaged to represent performance for that set of parameters. If the flow speed is too low, the fin will traverse the entire

\footnotetext{
${ }^{3}$ In post-processing the data, the first 2 flapping cycles are omitted to eliminate any transient effects, and thus cycle-averaged measurements are taken over 10 full flapping cycles.
} 
rail distance in a small number of cycles, reducing the duration of constant-velocity swimming over which measurements can be taken. If the flow speed is too high, the fin will not propel itself upstream at all. Therefore appropriate flow speeds were found through iteration in order to provide a minimum of 5 full cycles over the length of the rail (although most tests completed at least 7 cycles) This procedure was carried out 5 times for each set of parameters to provide mean values and standard deviations. The basic setup for both types of testing is shown in figure 5.3 .

In addition to tests performed in the water tunnel, the power input required to flap the fin in air is measured so that it can be subtracted from the power required to flap the fin in water (for the same set of frequency and amplitude parameters) in order to calculate the net power to the fluid. Since the angular velocity is prescribed at each instant for a set of parameters, the difference in power for water and air tests is caused by the amount of torque required to actuate the fin. The power to flap in air represents the torque needed to deform the skin material, overcome any friction in the structure, and the effort required to accelerate the mass of the fin throughout the flapping motion. Additional torque is needed when flapping in water, in order to impart energy to the fluid through momentum transfer. The difference between these two cycle-averaged power measurements is the net power input to the fluid, which is the measurement of interest for calculating both efficiency and economy (since only the net power input to the fluid can contribute to thrust production).

The fin was initially flapped with a given frequency and amplitude in 8 different orientations, all with the plane of the fin perpendicular to the plane of the ground, in order to find the orientation which required least power (thus giving the most conservative estimate of net power). The orientation requiring least power to flap in air was that with the tensegrity beam parallel to the ground, as shown in figure 5.4. All air power tests were performed in this orientation for each set of parameters $\{f, A\}$ corresponding to tests carried out in the water tunnel.

\footnotetext{
${ }^{4}$ Similar to the constrained tests, in post-processing the first few cycles of data are omitted from the calculation of cycle-averaged velocity for each test.
} 


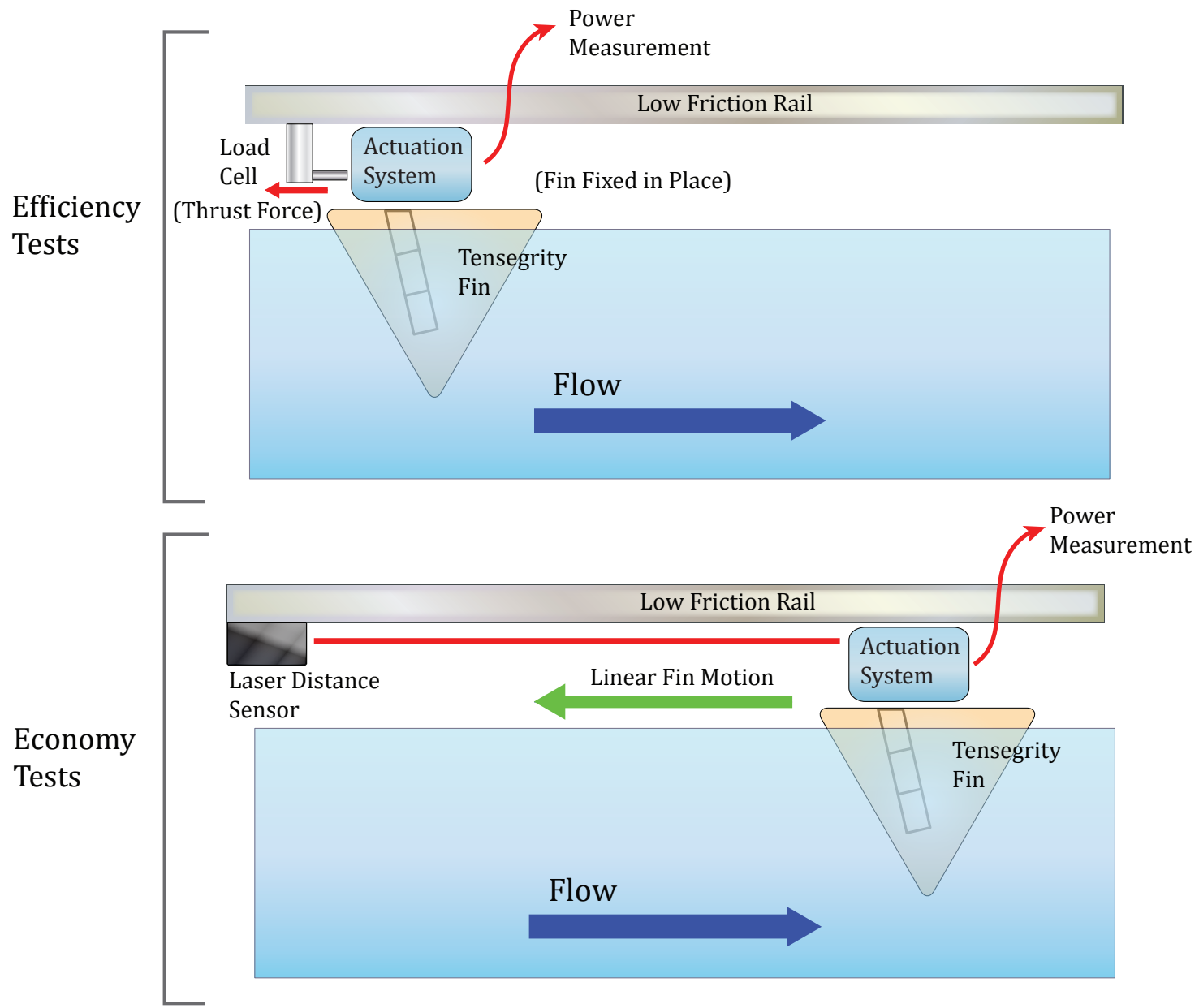

Figure 5.3: Schematic showing the two types of hydrodynamic tests performed: the setup for constrained efficiency tests, with the fin producing thrust against against a load cell at a fixed flow speed (top image); and the setup for unconstrained economy tests, with the fin allowed to free-swim upstream (bottom image). 


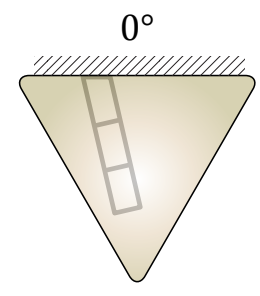

$180^{\circ}$

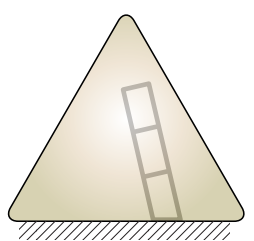

$12^{\circ}$

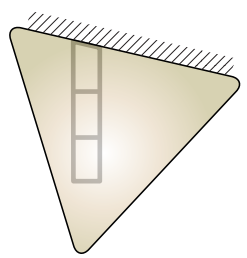

$192^{\circ}$

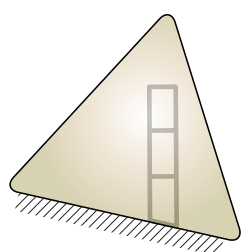

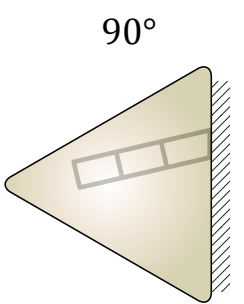

$270^{\circ}$

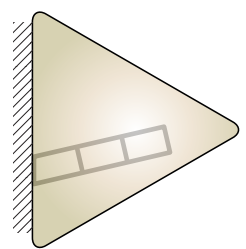

$102^{\circ}$

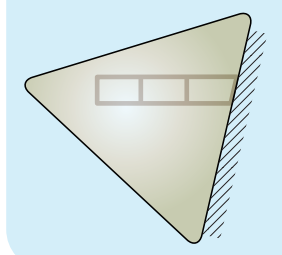

$282^{\circ}$

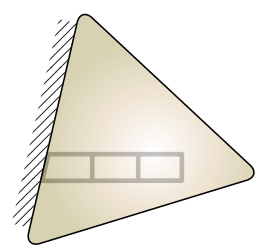

Figure 5.4: $P_{\text {air }}$ was initially measured in 8 different orientations to find one using the lowest power. Four of the orientations place the root chord either horizontal or vertical and four of the orientations place the tensegrity beam either horizontal or vertical (note the beam is tilted back from the span-wise axis by $\phi=12^{\circ}$ ). The orientation at $102^{\circ}$ (tensegrity beam horizontal, leading edge up) was found to consume the lowest power to flap in air, and was used for the remainder of air power tests.

\subsubsection{Performance Calculations}

The raw data collected from both the water tunnel and air power experiments is post-processed in MATLAB to calculate swimming performance. For both constrained and unconstrained tests and for air power measurements, the instantaneous mechanical power of the tensegrity fin, $P_{i}$, is calculated as the product of the measured torque, $\tau_{i}$, and the angular velocity of the drive shaft, $\omega_{i}$, so that: $P_{i}=\tau_{i} \omega_{i}$ (each corresponding to the $i$ th sample of the test). The angular velocity is simply the derivative of the drive shaft's angular position with respect to time (where the shaft position is measured with a rotary potentiometer, as explained in section 4.4.), calculated as a centralized finite difference: $\omega_{i}=\frac{\theta_{i+1}-\theta_{i-1}}{2 d t}$. For each test, the instantaneous power is then averaged over the test duration (of $n$ samples) to give the cycle-averaged power: $\bar{P}=\sum P_{i} / n$. This calculation is performed for both the air tests and water tunnel tests so that the net power (for matching $f$ and $A$ ) is $\bar{P}_{\text {net }}=\bar{P}_{\text {water }}-\bar{P}_{\text {air }}$. For both types of water tunnel tests, the net power measurement 
can be nondimensionalized as the coefficient of power:

$$
C_{P}=\frac{\bar{P}_{\text {net }}}{\frac{1}{2} \rho S U^{3}}
$$

Here $\rho$ is the density of the fluid 5 is the planform surface area of the fin $\left(S=0.0252 \mathrm{~m}^{2}\right)$, and $U$ is the flow speed for each kind of test. For constrained tests, $U$ is recorded from the digital readout of the water tunnel's flow sensor. For the unconstrained tests, the flow speed experienced by the free-swimming fin is the water tunnel speed plus the speed at which the fin travels upstream along the rail: $\bar{U}_{\text {free }}=U_{\text {tunnel }}+\bar{U}_{\text {fin }}$. The instantaneous speed of the fin along the rail is the derivative of its position, $y_{i}$, (measured with the laser distance sensor) with respect to time, calculated as a centralized finite difference: $U_{\text {fin }, i}=\frac{y_{i+1}-y_{i-1}}{2 d t}$. The speed used for performance calculations is the average of this instantaneous speed, taken over $n$ samples: $\bar{U}_{\text {fin }}=\sum U_{\text {fin }, i} / n$. Again, the sampling period is a subset of the original test data with the initial acceleratory portion removed, so that only constant-velocity free swimming is measured.

In constrained experiments, the instantaneous thrust measurement from the load cell is $T_{i}$, which is averaged over the test duration to give the cycle-averaged thrust for each constrained test: $\bar{T}=\sum T_{i} / n$. This thrust can be nondimensionalized as the coefficient of thrust by:

$$
C_{T}=\frac{\bar{T}}{\frac{1}{2} \rho S U^{2}}
$$

The nondimensional measures $C_{P}$ and $C_{T}$ are useful because they give an indication of power and thrust magnitudes if the fin size or flow speed were to be scaled. Additionally, they allow for the comparison of the thrust produced and power input

\footnotetext{
${ }^{5} \mathrm{~A}$ standard value for water, $\rho=1000 \mathrm{~kg} / \mathrm{m}^{3}$, is assumed since it is expected that the density would vary by less than $0.2 \%$ over the widest imaginable temperature range for the water tunnel.
} 
for this particular fin with other artificial fins in the literature [40, 41].

The Froude propulsive efficiency can then be calculated for constrained tests as the ratio of these two coefficients:

$$
\eta_{\mathrm{F}}=\frac{C_{T}}{C_{P}}=\frac{\bar{T} U}{\bar{P}_{\mathrm{net}}}
$$

As discussed earlier in this chapter and in section 2.2, this measure of efficiency represents the ratio of useful power output to the net power input to the fluid, and has been widely used in studies of oscillating propulsion. Each of the individual measurements used to calculate this efficiency has an uncertainty associated with it, therefore the uncertainty in the efficiency measurement itself must calculated using a standard propagation of error [127]. Thus the standard deviation of the propulsive efficiency measurements is calculated as:

$$
\begin{aligned}
\sigma_{\eta_{\mathrm{F}}} & =\sqrt{\left(\frac{\partial \eta_{\mathrm{F}}}{\partial \bar{T}} \sigma_{\bar{T}}\right)^{2}+\left(\frac{\partial \eta_{\mathrm{F}}}{\partial U} \sigma_{U}\right)^{2}+\left(\frac{\partial \eta_{\mathrm{F}}}{\partial \bar{P}_{\mathrm{net}}} \sigma_{\bar{P}_{\mathrm{net}}}\right)^{2}} \\
& =\frac{1}{\bar{P}_{\mathrm{net}}} \sqrt{\left(U \sigma_{\bar{T}}\right)^{2}+\left(\bar{T} \sigma_{U}\right)^{2}+\left(\frac{\bar{T} U}{\bar{P}_{\mathrm{net}}} \sigma_{\bar{P}_{\mathrm{net}}}\right)^{2}}
\end{aligned}
$$

Where $\sigma_{\bar{T}}, \sigma_{U}$, and $\sigma_{\bar{P}_{\text {net }}}$ are the standard deviations of the thrust, flow speed, and power measurements, respectively. This shows that as a the net power input to the fluid becomes small (typically for low flapping frequencies), the uncertainty in propulsive efficiency inherently increases.

For the unconstrained tests, it is desirable to compare the free-swimming speed with the power input required to produce that speed, so free-swimming economy of the fin is calculated as:

$$
\xi=\frac{\bar{U}_{\text {free }}}{\bar{P}_{\text {net }}}
$$


The result of this metric is a quantification of distance traveled per unit energy input $(\mathrm{m} / \mathrm{J}$ in SI units). The uncertainty in the economy measurements can be calculated in the same manner as above for the efficiency, so the standard deviation in economy measurements is:

$$
\sigma_{\xi}=\frac{1}{\bar{P}_{\text {net }}} \sqrt{\left(\sigma_{\bar{U}_{\text {free }}}\right)^{2}+\left(\frac{\bar{U}_{\text {free }}}{\bar{P}_{\text {net }}} \sigma_{\bar{P}_{\text {net }}}\right)^{2}}
$$

Where $\sigma_{\bar{U}_{\text {free }}}$ is the standard deviation in the free-swimming velocity measurements. Similar to the uncertainty in efficiency, this shows that as a the net power input to the fluid becomes small (typically for low flapping frequencies), the uncertainty in free-swimming economy inherently increases.

As discussed in section 2.2.1, the Strouhal number is an important measure of nondimensional frequency often used to characterize oscillatory swimming. The Strouhal number is calculated as:

$$
S t=\frac{f A}{U}
$$

Here $f$ is the flapping frequency and $U$ is the fin speed (as defined above for either constrained or unconstrained tests). The amplitude, A, used for Strouhal number is intended to represent the size of the wake, which is fairly straightforward for two-dimensional flows. However it is difficult to estimate a priori for this case because the amplitude of the fin varies along the span and so the wake should be highly three-dimensional. To estimate this amplitude, we can assume the peak-topeak amplitude at each point along the span represents the wake width there. In this way, the wake width at the fin root is zero and the wake width at the tip is the peak-to-peak amplitude, which is $2 A_{\text {tip }}$, or twice the normal tip amplitude. Therefore 
the average wake width along the span should be about half the wake width at the tip, or simply $A_{\text {tip }}$, the tip amplitude of the fin. This convention has been adopted in [4, 41], and should provide estimates of St consistent with those and other similar studies. Uncertainty in Strouhal number measurements can also be estimated with a propagation of error, considering the uncertainties in amplitude and flow speed measurements 6 The standard deviation of these measurements is then calculated as:

$$
\sigma_{S t}=\frac{f}{U} \sqrt{\sigma_{A}{ }^{2}+\left(\frac{A}{U} \sigma_{U}\right)^{2}}
$$

Where $\sigma_{A}$ is the standard deviation in amplitude measurements (explained next) and $\sigma_{U}$ is the standard deviation in flow speed measurements, which is either a constant value $(1.5 \mathrm{~mm} / \mathrm{s}$ as mentioned previously) for constrained tests, or is calculated as the standard deviation in free-swimming speed measurements across the number of unconstrained tests.

\subsection{Results and Discussion}

\subsubsection{Amplitude Measurements}

Due to variability in the fluid loading (depending on flapping parameters) and its effect on resulting flapping amplitudes, the actual fin tip amplitudes were measured by optical analysis (similar to the in-air static amplitude validations) over the range of frequencies used for the flapping experiments. From a posterior view of the fin (behind the test section of the water tunnel, looking upstream), the tip excursions from the neutral plane were recorded using long-exposure photographs for each flapping frequency while the fin was constrained along the rail. The tip-to-tip amplitude $\left(2 A_{\text {tip }}\right)$ for each test was measured from these photographs, as shown in figure

\footnotetext{
${ }^{6} \mathrm{It}$ is assumed that the flapping frequency is exact, and frequency analysis of the measured signals indicates this is a sound assumption.
} 

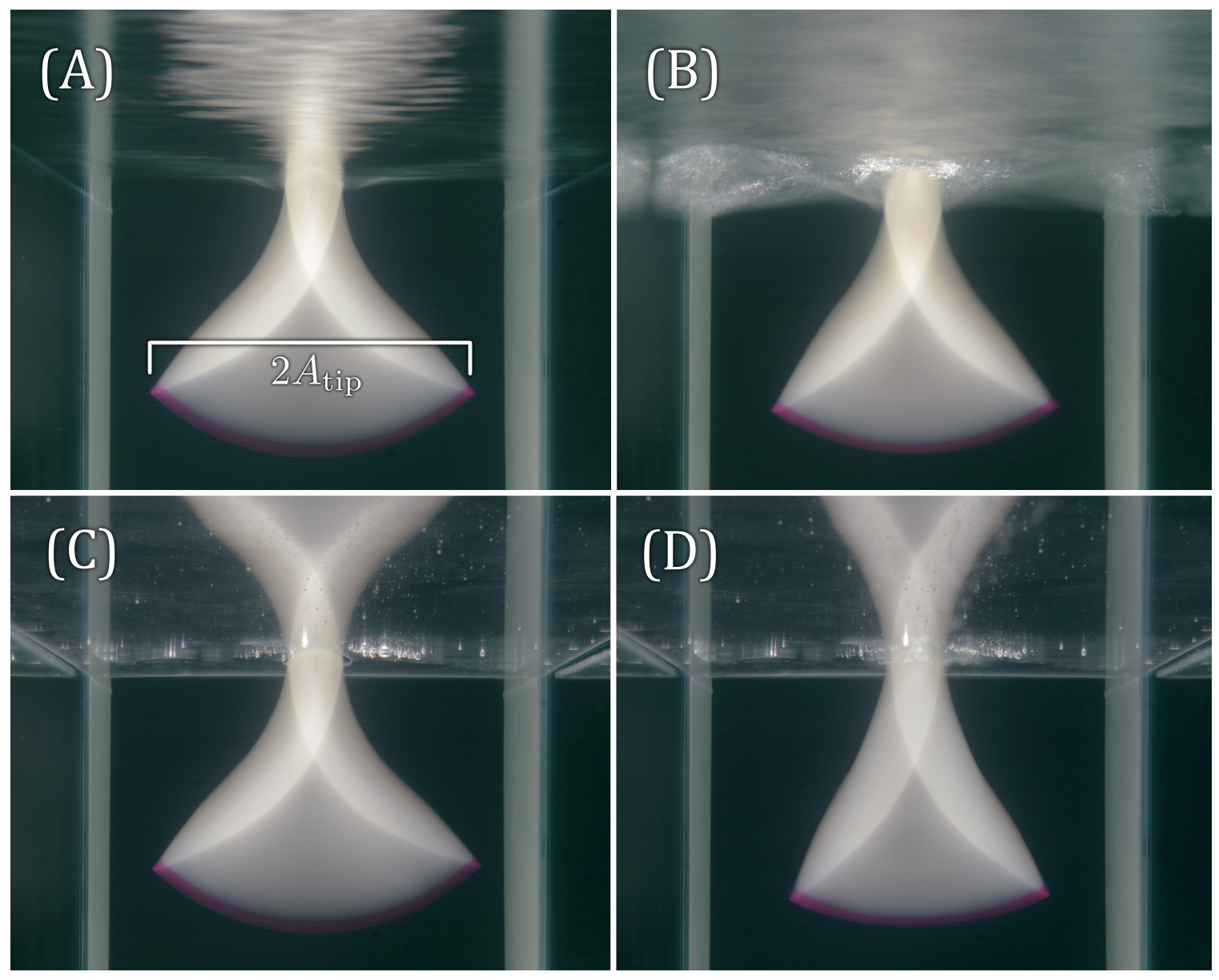

Figure 5.5: Measurement of fin tip amplitudes using posterior view long-exposure photographs. (A) $f=0.5 \mathrm{~Hz}$, free surface in the water tunnel, with tip-to-tip amplitude marked. (B) $f=1.3 \mathrm{~Hz}$, free surface. (C) $f=0.5 \mathrm{~Hz}$, wave suppression plate applied to water surface. (D) $f=1.3 \mathrm{~Hz}$, wave suppression plate applied. All images for a nominal amplitude of $A / b=0.67$ and at a flow speed of $1 \mathrm{BL} / \mathrm{s}$.

5.5. This value is divided in half to provide the amplitude, $A$, for Strouhal number calculations.

This procedure was performed both with and without the surface wave suppression plate applied to the free surface of the water for a range of flow speeds, and the recorded amplitudes demonstrated several results. First, as expected, the mechanical system comprising the fin (artificial skin, underlying tensegrity beam, and servomotor driving the actuation) exhibited a dynamic frequency response in which the actual fin amplitude generally decreased with increasing flapping frequency. Second, when the free surface of the water tunnel is left unconstrained, the flapping fin is capable 
of generating significant surface waves, an effect which itself had a notable frequency dependence. Third, the occurrence of a coupled resonance between the fin and the free surface of the water tunnel resulted in a local peak in fin tip amplitude. Fourth, application of the surface wave suppression plate effectively eliminated the two previously mentioned surface wave effects, resulting in monotonically decreasing fin tip amplitude with increasing flapping frequency.

Figure 5.6 shows the measured tip amplitudes for 3 flow speeds, both with and without the surface wave suppression plate applied. The nominal amplitude - meaning the analytical amplitude predicted by the amount of rotation sent as an instruction to the servomotor (see section 4.5) - for these tests was $A / b=0.67$. Note that the flow speeds have been normalized by the root chord length of the fin, which represents the "body length" of the fin $(0.248 \mathrm{~m})$, providing a useful, biologically-relevant reference for flow speed, so "BL/s" (body lengths per second) will be used throughout the results to report flow speeds. When the surface wave suppression plate is not applied, the resulting tip amplitudes generally decrease with increasing flapping frequency, until some critical frequency where there is a local peak in amplitude. These local peaks correspond with significant (up to $3.3 \mathrm{~cm}$ peak-to-peak, measured at the tunnel wall) observed surface waves across the tunnel width. At these critical frequencies, the heaving motion of the fin excites a resonance of the surface waves in the water tunnel and this coupled resonance results in increased fin amplitudes (compared to neighboring frequencies). When the surface wave suppression plate is applied, the surface wave resonance effect is eliminated and fin amplitudes decrease monotonically with increasing frequency. Also, fin amplitudes were slightly higher in general when the surface plate was applied.

For both sets of tests there was a correlation between fin amplitude and imposed flow speed, where a larger flow speed generally decreased the fin amplitude. The flow speed also influenced the critical frequency of the surface wave resonance in the three flow speeds tested: as flow speed increased, the resonant frequency decreased. 

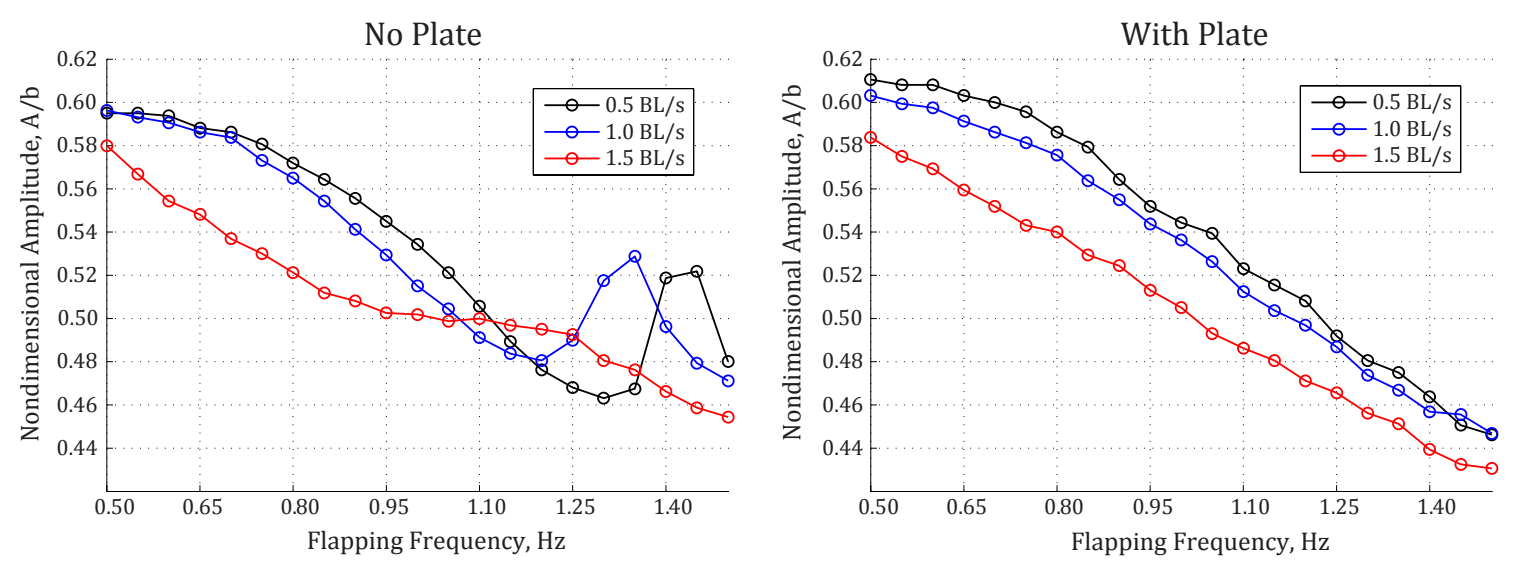

Figure 5.6: Actual fin tip amplitudes over a range of frequencies, at 3 different flow speeds, both with a free surface in the water tunnel (left) and with the surface wave suppression plate applied (right). All tests were for a nominal amplitude of $A / b=$ 0.67 .

For $U=0.5 \mathrm{BL} / \mathrm{s}$, the resonant frequency is between 1.40 and $1.45 \mathrm{~Hz}$ and for $U=1.0 \mathrm{BL} / \mathrm{s}$, the resonant frequency is between 1.30 and $1.35 \mathrm{~Hz}$. For $U=1.5 \mathrm{BL} / \mathrm{s}$, the resonant frequency appears between 1.05 and $1.25 \mathrm{~Hz}$, although the effect was much less pronounced at this high flow speed as the lateral momentum (in the heave direction) imparted to the fluid was swept downstream away from the fin more rapidly. Correspondingly, the observed maximum surface wave amplitudes were less significant at this high flow speed.

Further tests were conducted with the surface wave suppression plate in place, over an even larger range of speeds and with higher resolution between the speeds. Again, the results were consistent, in the sense that the plate was effective at eliminating effects from any surface disturbances, so that amplitudes dropped with increasing flapping frequency. Figure 5.7 shows the results over a range of speeds from $0.5 \mathrm{BL} / \mathrm{s}$ to $2.0 \mathrm{BL} / \mathrm{s}$. As before, across the range of frequencies increasing flow speed was generally correlated with lower amplitudes. This figure also shows that over a wide range of frequencies and flow speeds, a nominal amplitude of $A / b=0.67$ results in actual amplitudes centered around $A / b \approx 0.5$, which is comparable to biological observations of oscillatory batoid rays. 


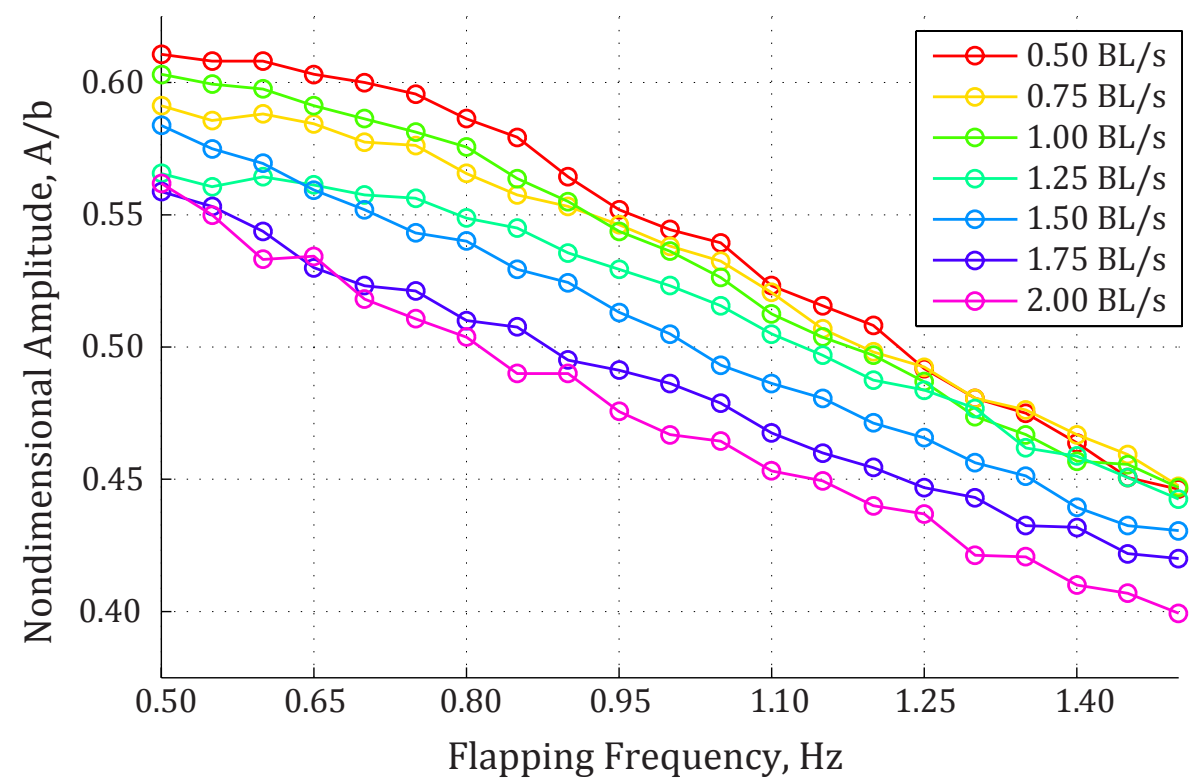

Figure 5.7: Actual fin tip amplitudes over a wide range of flow speeds. All tests were for a nominal amplitude of $A / b=0.67$. Across a range of frequencies, actual fin amplitude generally decreases with increasing flow speed.

This data set of amplitudes is used in the determination of Strouhal number for constrained efficiency tests (both with and without the wave suppression plate). In unconstrained tests, the fin moves along the rail so the same optical measurement method cannot be used (the fin amplitude would appear to decrease as the fin moved away from the camera due to perspective). Also the effective flow speed in unconstrained tests cannot be completely prescribed, so instead these optical amplitude measurements can be used to create an interpolation surface, from which the actual amplitude is estimated using the pair of $\{f, U\}$ for each unconstrained test. From repeated tests, the standard deviation in these measurements (both from optical measurement error and from variability in actual fin amplitude from test to test) is estimated to be about $13 \mathrm{~mm}$.

Using equation 5.8 we can now estimate the uncertainty in calculated Strouhal number over the parameter range of interest. The form of equation 5.8 suggests that the standard deviation in $S t$ will be maximized when $f$ is high, $A$ is high, and $U$ is low (this corresponds to a large value of $S t$ ). So for $f=1.5 \mathrm{~Hz}, A=0.14 \mathrm{~m}$, and 
$U=0.12 \mathrm{~m} / \mathrm{s}$ (giving a high Strouhal number of 1.75 ), we have $\sigma_{S t}=0.16$, or about 13\%. Conversely, the standard deviation in Strouhal number will be minimized for low $f$, low $A$, and high $U$. For example with $f=0.5 \mathrm{~Hz}, A=0.08 \mathrm{~m}$, and $U=0.37 \mathrm{~m} / \mathrm{s}$ (giving a low Strouhal number of 0.11 ), we have $\sigma_{S t}=0.02$, or about $0.8 \%$. Given a more biologically-relevant set of parameters for this fin, such as $f=0.9 \mathrm{~Hz}, A=0.1 \mathrm{~m}$, and $U=0.25 \mathrm{~m} / \mathrm{s}$ (giving a Strouhal number of 0.36 ), then we have $\sigma_{S t}=0.05$, or about $2 \%$.

This section has described both the frequency response and the effect of surface waves on actual fin amplitudes. Results show that while surface wave effects can be considerable, they can be accounted for in terms of Strouhal number (since the amplitudes can be measured) and the effect can be eliminated through surface wave suppression. In the next section, the effect of surface waves on power, thrust, and efficiency measurements will be considered.

\subsubsection{Constrained Tests: Surface Wave Suppression}

Constrained water tunnel tests were performed over a range of flapping frequencies from 0.5 to $1.5 \mathrm{~Hz}$ at a nominal amplitude of $A / b=0.67$. A flow speed of $0.248 \mathrm{~m} / \mathrm{s}$ (1.0 BL/s, normalized by root chord) was chosen to give a nominal Strouhal number range from about 0.27 to 0.82 . Tests with these parameters were performed both with and without the use of the surface wave suppression plate (hereafter referred to as "the plate" for brevity) to determine the influence on swimming performance measurements of flapping near a free surface in a water tunnel of limited width.

The power input to the fluid during these flapping experiments is captured by the measurement of cycle-averaged net power, but first the cycle-averaged gross power for each test is calculated from torque and angular velocity. Figure 5.8 shows a sample of instantaneous torque and angular velocity measurements used to calculate the gross power in a test with the plate on. In this plot, signals have been filtered with a Fourier transform (FFT) to remove broadband noise. Across all frequencies 


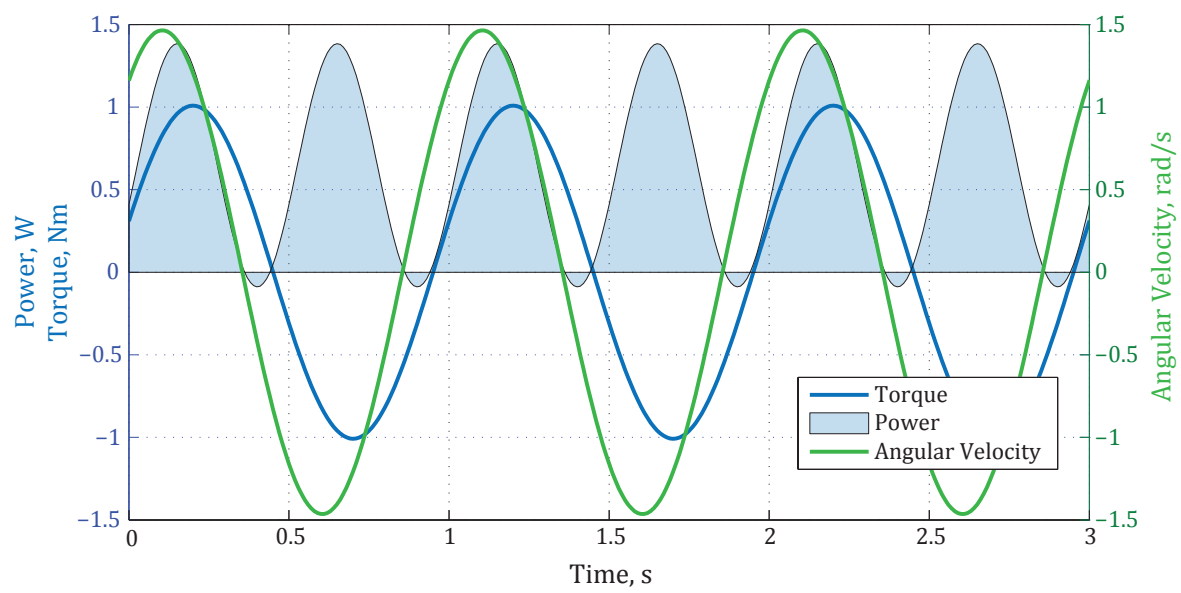

Figure 5.8: Instantaneous torque, angular velocity, and gross power measurements superimposed (FFT filtered), for $f=1.0 \mathrm{~Hz}$ with the plate on. There is a small period of negative power that occurs twice per flapping cycle, corresponding to the reversal of the fin at each extreme of its travel.

there was no qualitative change in the torque signals, only the magnitudes varied, so a representative sample at $1.0 \mathrm{~Hz}$ is shown. The oscillating torque signal has a constant phase lag behind the angular velocity of the actuation system, indicating maximum torque output occurred just after passing through the neutral plane each half-cycle. Also as a result of this phase lag, there is a period in every half-cycle when the power measurement becomes negative, corresponding to the applied torque being opposite the direction of rotation, which occurs just after the fin changes direction at its maximum amplitude. The negative power can be attributed most likely to an added-mass effect of the fluid: a mass of fluid following the fin must decelerate against the fin as it changes direction.

Figure 5.9 shows the total (gross) cycle-averaged mechanical power during water tunnel tests, the cycle-averaged power to flap in air at the same amplitude and frequencies, and also the cycle-averaged net power to the fluid (the difference between total power and air power). At the lowest frequencies, the measured net power is quite small (on the order of $0.1 \mathrm{~W}$ ) and the net power to flap in water increases considerably with frequency. This is not surprising since the fluid loading estimate of section 4.3 predicts the loading should scale with the square of flapping frequency. 
Interestingly, in the lower half of the testing frequency range, the power required to flap with the plate on is actually slightly higher than the power required to flap without the plate. Starting at around $1.1 \mathrm{~Hz}$, the effect of the water tunnel surface waves becomes evident: the total power, and therefore net power, without the plate drops considerably compared to the power with the plate. This decrease in power without the plate corresponds to observed surface wave resonance (maximum amplitude). The local minimum in power around 1.25-1.3 Hz matches closely with the local maximum amplification in fin amplitude (see figure 5.6), meaning the surface wave resonance simultaneously increases the fin amplitude while decreasing the power necessary to reach that amplitude. This indicates that the mass of water oscillating laterally across the tank is actually assisting the fin flapping motion. Meanwhile, the net power with the plate applied increases monotonically as predicted by the fluid loading model and shows none of the surface wave resonance effects. Beyond the resonant frequency (around 1.4-1.5 Hz), the net power without the plate rejoins the trend in net power with the plate.

The net thrust for these experiments derives from an average over several cycles of the instantaneous thrust signals. Figure 5.10 shows a representative sample of the instantaneous thrust signals from the load cell for all frequencies tested at $U=1.0 \mathrm{BL} / \mathrm{s}$ with the plate off (signals FFT filtered for display). Positive thrust is generated throughout most of the flapping cycles above about $0.8 \mathrm{~Hz}$, however each of the signals dips into negative net thrust (drag) for every frequency. This plot also clearly shows two peaks in thrust per flapping cycle, corresponding to vortex shedding observed during testing. Results are similar for the case with the plate on, just with different magnitudes for the thrust (which are explained in the cycle-averaged measurements).

The oscillatory thrust signals can be spectrally analyzed using a Fourier transform to examine magnitudes of the frequency components. Figure 5.11 shows a distinct peak in thrust at twice the forcing frequency, again corresponding with the thrust 


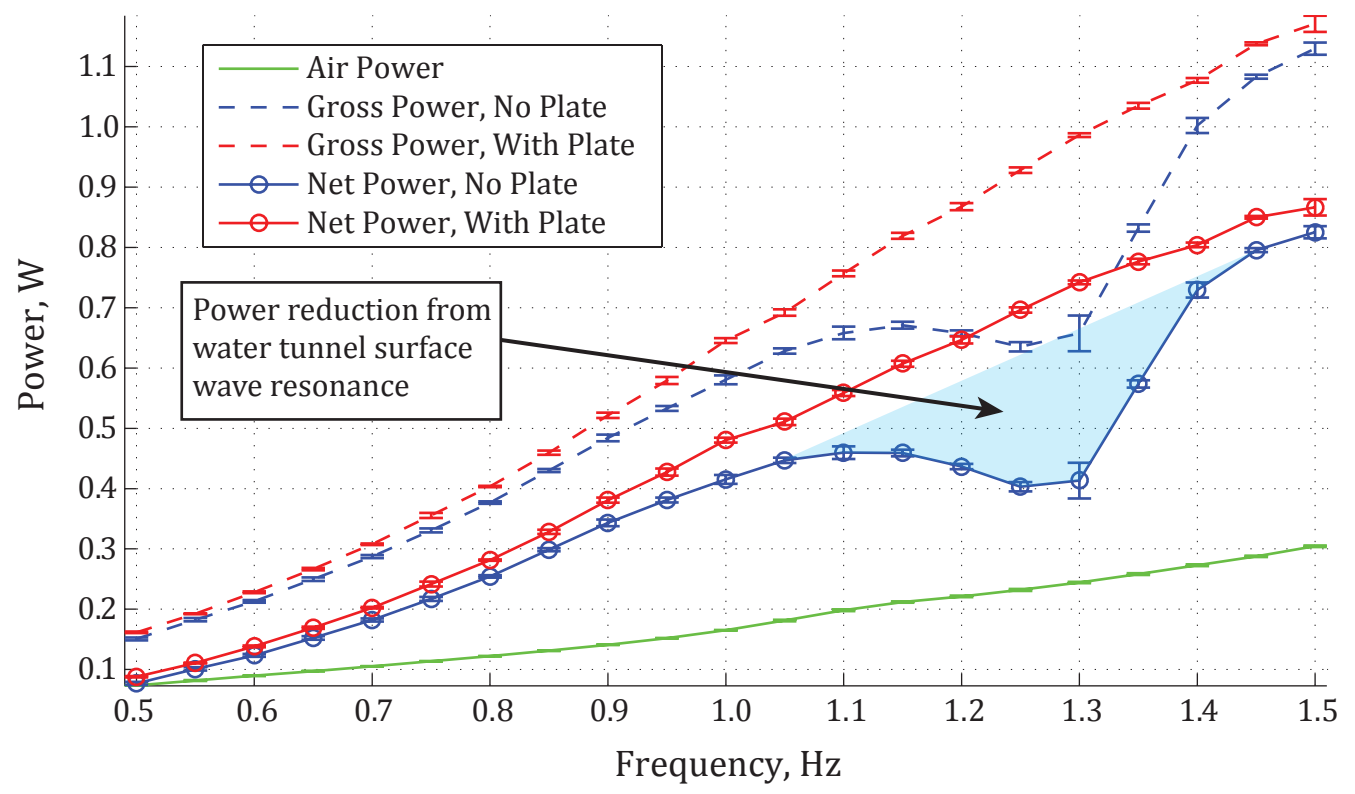

Figure 5.9: Cycle-averaged power measurements both with and without the wave suppression plate, taken at $U=1.0 \mathrm{BL} / \mathrm{s}$ and $A / b=0.67$. Net power to the fluid is the gross mechanical power minus the power to flap the fin in air. Error bars reflect the standard deviation from 5 tests.

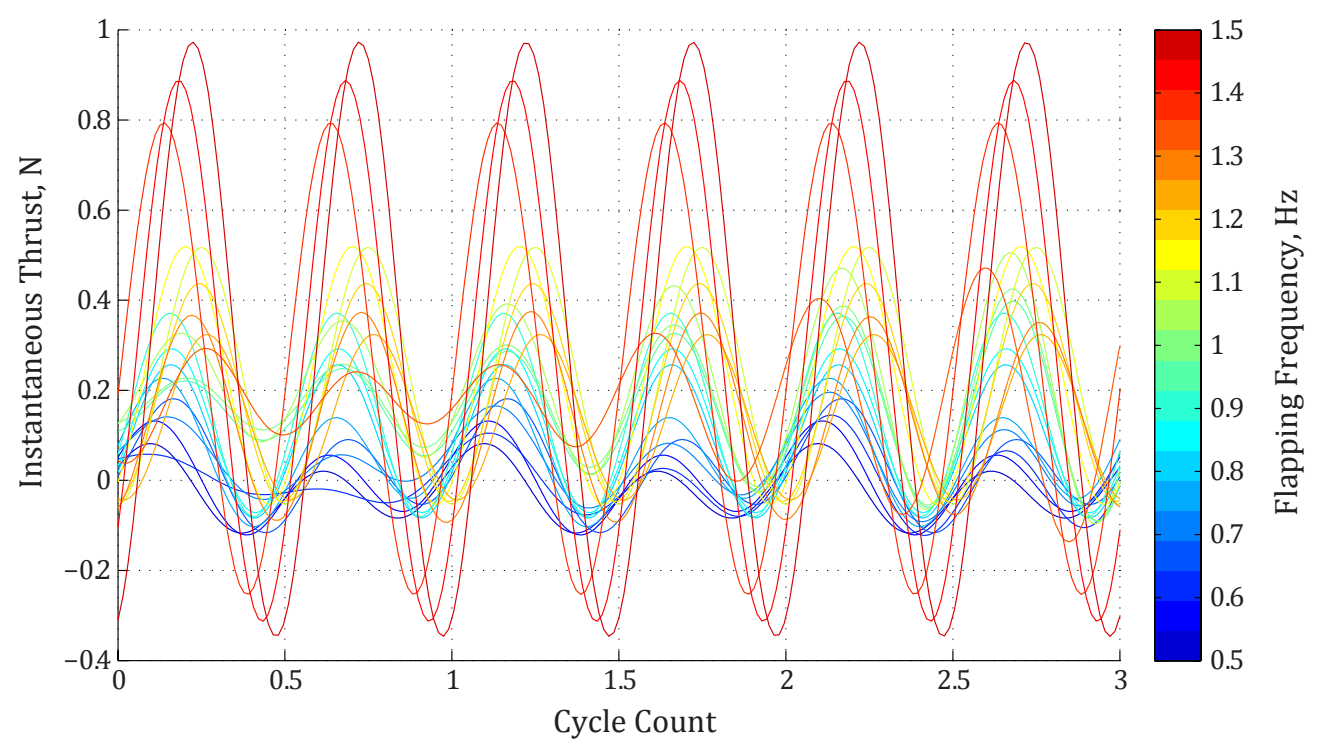

Figure 5.10: Instantaneous thrust signals for all flapping frequencies with a free surface in the water tunnel (FFT filtered to remove signal noise), showing the magnification in thrust peaks as frequency increases. Note that the phase angle for each frequency does not necessarily align because of the way sampling is performed during data capture. 


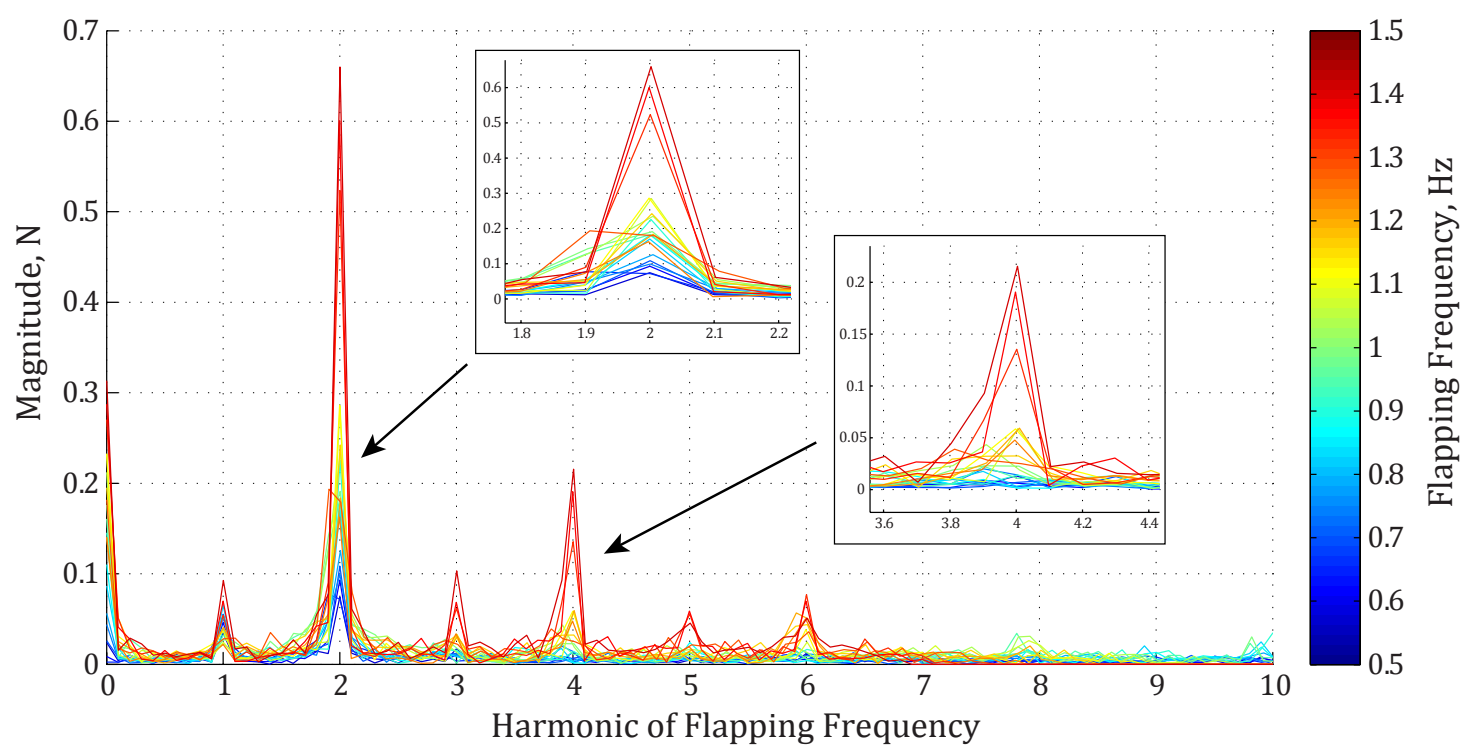

Figure 5.11: Magnitude of thrust over the frequency spectrum. The peak at the first harmonic shows magnitudes increasing with frequency until reaching a saturation point. The second harmonic shows a much stronger contribution to thrust, especially at higher frequencies. Also the DC bias, corresponding to the cycle-averaged thrust, is present on the far left.

peaks seen every half-cycle in the time domain signals. Thrust peaks are also observed at several harmonics of the fundamental frequency, with the second highest peak occurring at four times the flapping frequency. The peaks in thrust are noticeably larger for the top few frequencies (this effect is explained by examining surface wave effects, which are discussed next). The 0th harmonic (DC component) represents the cycle-averaged thrust signal for each frequency.

The cycle-averaged thrust measurements for this set of tests at $1.0 \mathrm{BL} / \mathrm{s}$ is shown in figure 5.12. For the lower portion of the frequency range, the thrust produced in both cases (with and without the plate) was essentially the same (within the uncertainty range), with the fin producing net thrust for all frequencies above $0.6 \mathrm{~Hz}$ and below this frequency the result was net drag. As seen in the power measurements, the effect of free surface waves becomes apparent starting around $1.1 \mathrm{~Hz}$, at which point the net thrust drops for the test without the plate. There is a local minimum in net thrust around $1.3 \mathrm{~Hz}$, again corresponding to the maximum observed surface 


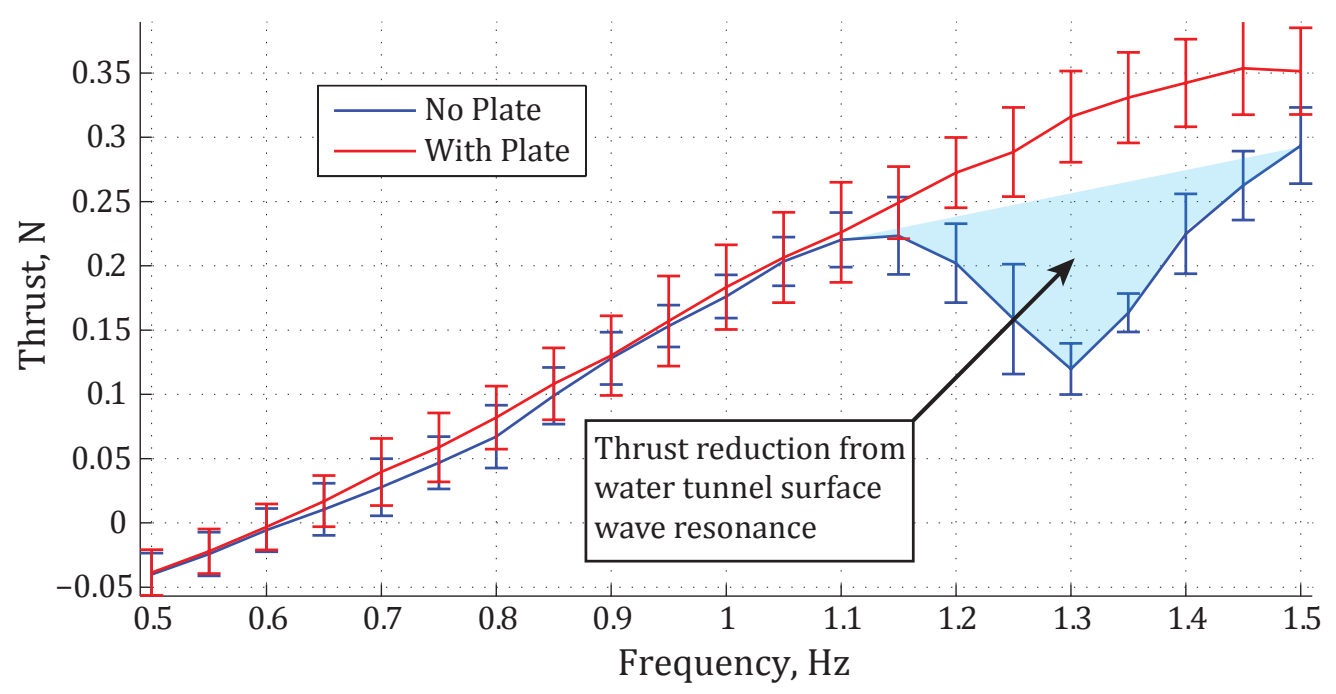

Figure 5.12: Cycle-averaged net thrust measurements for constrained water tunnel tests at $1.0 \mathrm{BL} / \mathrm{s}$ and $A / b=0.67$. Thrust production clearly drops around the surface wave resonant frequency for the case of a free surface in the water tunnel. Error bars reflect standard deviations from 5 sets of tests.

wave amplitude and local maximum of fin amplitude. The simplest explanation for this drop in thrust is that the lateral (heave direction) loading on the fin decreases as the fluid oscillates in resonance with the fin. The thrust produced by this fin is based on lift, which has components in the lateral (heave) direction and in the streamwise direction (thrust). So as the lift loading decreases (as indicated by the power measurements), the component of that lift load pointing upstream (the thrust) also decreases in magnitude. Meanwhile for the test set with the plate on, thrust increased monotonically with flapping frequency (until the very highest flapping frequency of 1.5 Hz where the thrust basically levels off - this could be attributed to the decrease in fin amplitude seen in earlier results), reaching a maximum of about $0.35 \mathrm{~N}$.

The Froude propulsive efficiency for these tests, calculated with equation 5.3 , is shown in figure 5.13. The first item to note about these results is that the efficiency is relatively low, peaking at about $12 \%$ for the case with no plate and at about $11 \%$ for the case with the plate. This low efficiency is somewhat counter to expectations, but will be discussed in more depth with a larger set of results in the next section. The other important thing to note about these results is that even though the power 


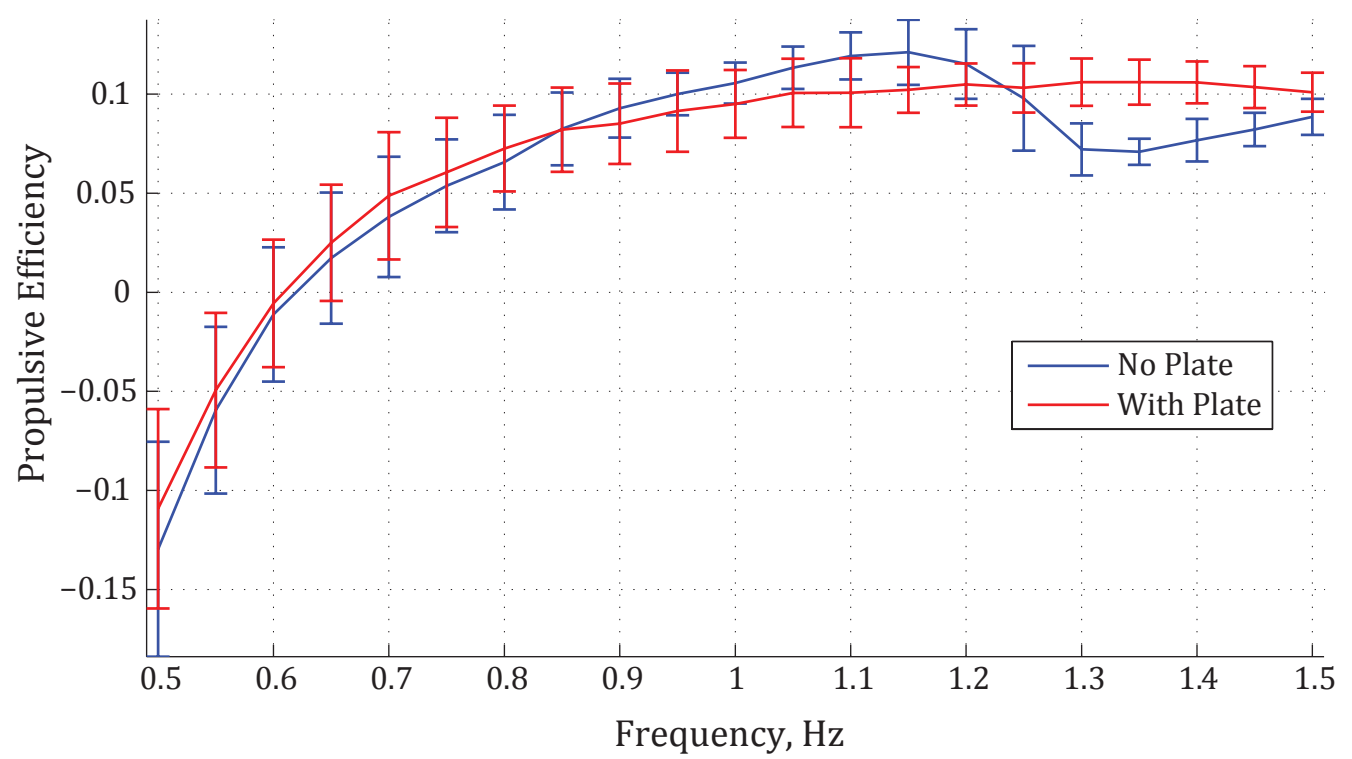

Figure 5.13: Resulting propulsive efficiency measurements for constrained water tunnel tests at $1.0 \mathrm{BL} / \mathrm{s}$ and $A / b=0.67$. Error bars reflect the propagated error in standard deviations from 5 sets of tests.

and thrust measurements are both reduced significantly by the presence of resonant free surface waves in the water tunnel, the decrease in both measures is proportional, so that the resulting efficiency is largely unaffected. Below about $1 \mathrm{~Hz}$ flapping, the efficiency results for both tests are essentially identical (certainly within the range of error). From about $1-1.25 \mathrm{~Hz}$, the efficiency with no plate is slightly higher than the efficiency with the plate (12\% vs. 10\%). This appears to come from the fact that the power input in this frequency range is slightly lower for the case with no plate (refer back to figure 5.9], as it starts to benefit from the surface waves without as much reduction in thrust. From about $1.25-1.5 \mathrm{~Hz}$ the efficiency with no plate is slightly lower than the efficiency with the plate (7\% vs. 11\%). In this range, for the case with no plate the thrust reaches a local minimum while the power input starts to climb again, so the resulting efficiency is slightly lower.

The efficiency results can also (more appropriately) be plotted against Strouhal number, as shown in figure 5.14. This plot demonstrates that in the range of interest for free swimming $(0.2<S t<0.4)$, there is no significant difference between the 


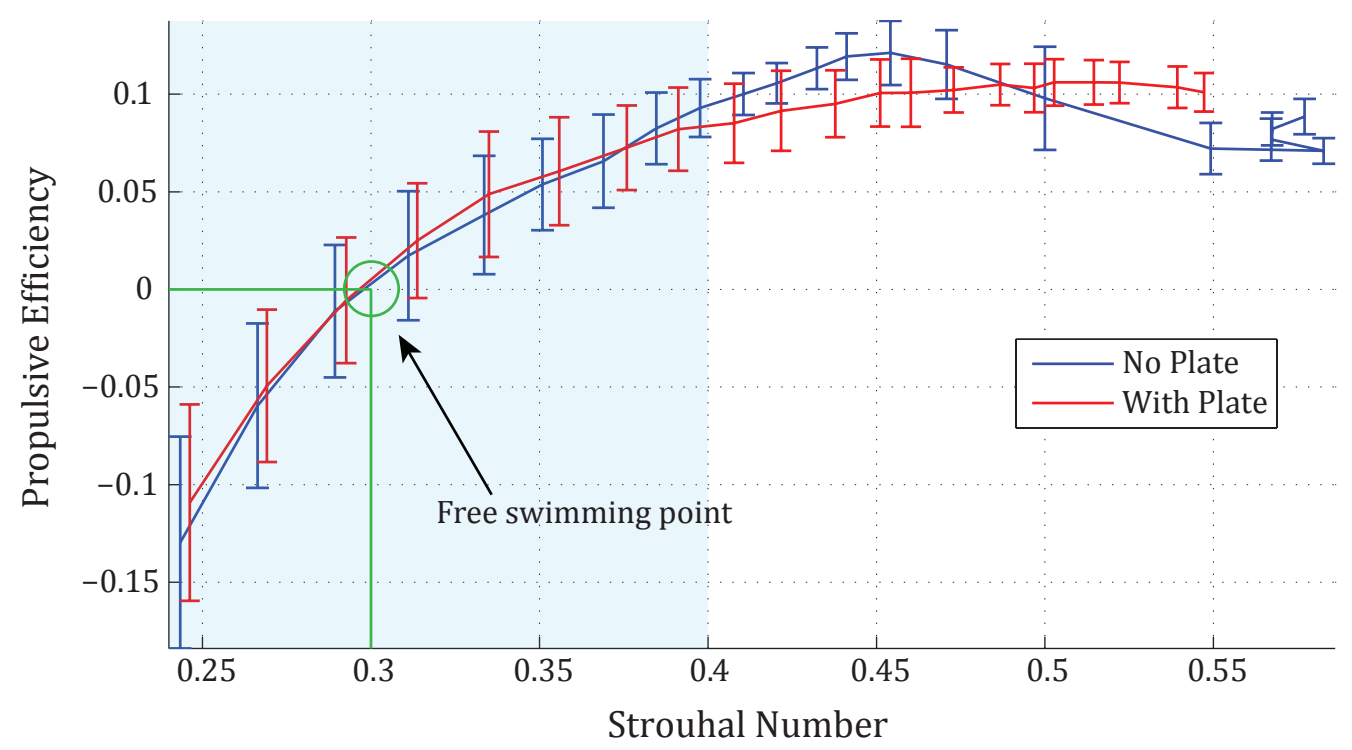

Figure 5.14: Resulting propulsive efficiency measurements for constrained water tunnel tests at $1.0 \mathrm{BL} / \mathrm{s}$ and $A / b=0.67$, plotted against Strouhal number. In the range expected for free swimming $(S t=0.2-0.4)$, the results are essentially identical.

efficiency results with and without the plate. Even in the higher Strouhal number range (where free swimming is not expected to occur), the difference in measured efficiencies is fairly small. This plot also shows that if this fin were freely swimming at $1.0 \mathrm{BL} / \mathrm{s}$, we should expect a Strouhal number of about 0.3 - since that is where there is zero net thrust — which matches well with the observed Strouhal number range for biological swimmers.

\subsubsection{Constrained Tests: Flow Speed Variation}

While the presence or absence of a free surface does not appear to make a significant difference in measured efficiency, further constrained tests were conducting with the wave suppression plate applied so that any surface wave effects did not further complicate the results. In this set of tests, the frequency range and amplitude are kept constant while the imposed flow speed is varied to see what effect this has on efficiency measurements. Five different flow speeds were used, ranging from 0.5 to $1.5 \mathrm{BL} / \mathrm{s}$, corresponding to a Reynolds number range of approximately 34,000 to 


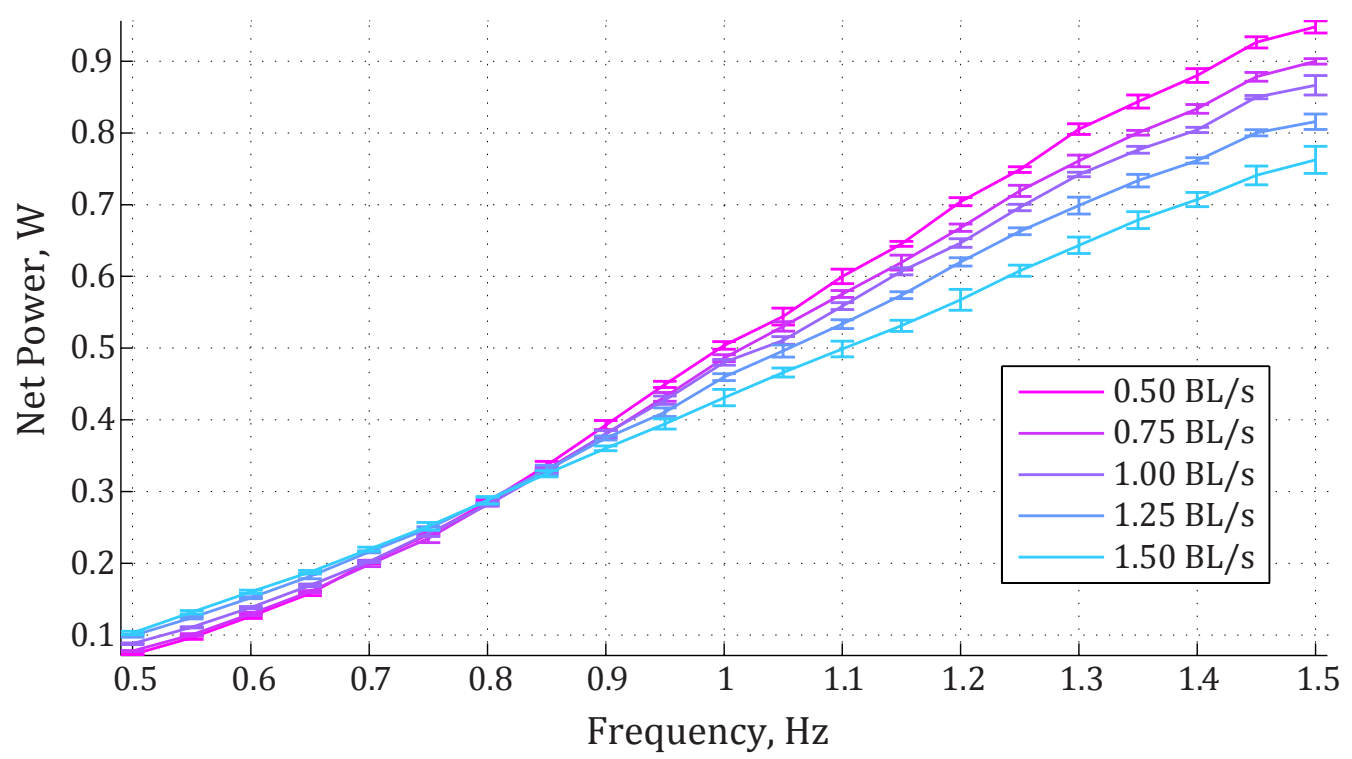

Figure 5.15: Net power to the fluid over a range of flow speeds. Error bars represent the standard deviation from 5 tests.

103,000 (based on the root chord length). Again a flapping frequency range of 0.5 to $1.5 \mathrm{~Hz}$ was used, with a nominal amplitude for all tests of $A / b=0.67$.

Figure 5.15 shows the cycle-averaged power measurements for these tests. At lower flapping frequencies (from about $0.5-0.8 \mathrm{~Hz}$ ) there is a slight increase in power input to the fluid as the flow speed increases. Interestingly, there is an inflection point at about $0.8 \mathrm{~Hz}$ where the power measurement seems to be independent of flow speed. Above this inflection point (from about $0.8-1.5 \mathrm{~Hz}$ ), there is a negative correlation between power and flow speed, in which lower flow speeds actually require more power input to the fluid.

The net power can be nondimensionalized as the coefficient of power (see equation 5.1 and plotted against Strouhal number, as shown in figure 5.16. Since Strouhal number and coefficient of power both scale inversely with flow speed $(\propto 1 / U$ and $\propto 1 / U^{3}$, respectively), the resulting values are both quite large for the lowest flow speed of $U=0.5 \mathrm{BL} / \mathrm{s}$. Overall this scaling shows that all the power results basically collapse onto a single curve. Only the power data from the slowest flow speed showed an exception: the functional relationship between $C_{P}$ and $S t$ seems consistent, just 


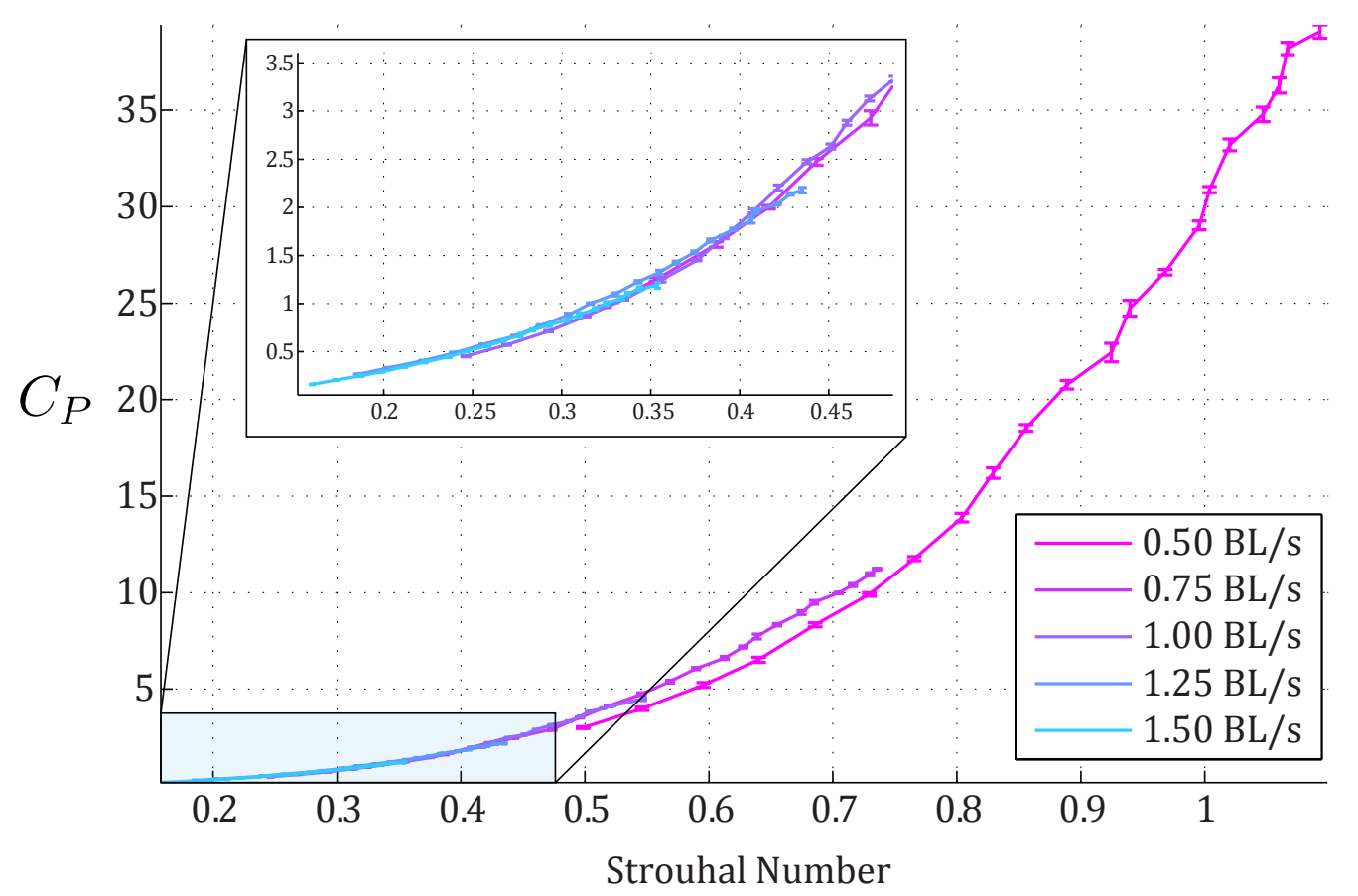

Figure 5.16: Coefficient of power over a range of flow speeds. Error bars represent the standard deviation from 5 tests.

offset slightly from the rest of the data.

Figure 5.17 shows the net thrust results for this set of tests over a range of flow speeds, which demonstrates that increasing flow speed effectively scales down the net thrust produced by the fin. For example, at the highest frequency tested $(1.5 \mathrm{~Hz})$, the net thrust at $U=0.5 \mathrm{BL} / \mathrm{s}$ is approximately $0.64 \mathrm{~N}$; if the flow speed is scaled up to $U=1.5 \mathrm{BL} / \mathrm{s}$ (by a factor of 3 ), the net thrust is scaled down to $0.18 \mathrm{~N}$ (a factor of $1 / 3.6)$. This reflects the idea that the net thrust produced by the fin should be proportional to the momentum added to the wake; as the imposed flow speed increases, the wake velocity relative to the surrounding flow is not as great, meaning net thrust is reduced.

This thrust data can be nondimensionalized as the coefficient of thrust (see equation 5.2 and the flapping frequency as Strouhal number, as shown in figure 5.18 . Similar to the coefficient of power, this effectively collapses the data down to a single curve, although not as cleanly. For one thing, there is more uncertainty in the thrust 


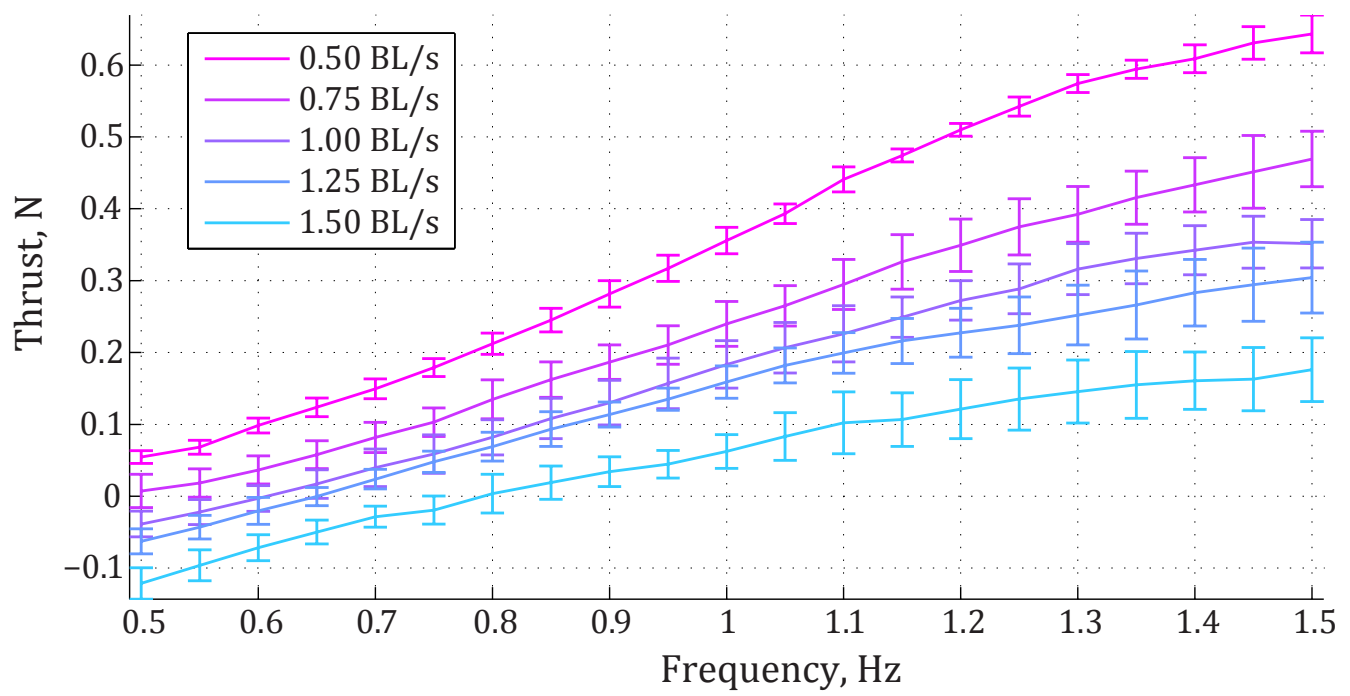

Figure 5.17: Cycle-averaged net thrust over a range of flow speeds. Error bars represent the standard deviation from 5 tests.

data (compared to the power data), so deviations from the single curve are somewhat expected. However there also seems to be a systematic trend which makes the coefficient of thrust slightly higher for tests performed at higher speeds. For example, we can compare the results along a vertical line drawn at $S t=0.3$ : for the flow speed of $U=1.0 \mathrm{BL} / \mathrm{s}, C_{T} \approx 0$; for the flow speed of $U=1.25 \mathrm{BL} / \mathrm{s}, C_{T} \approx 0.06$. This scaling reflects the fact that the tests each have a different Strouhal value where the net thrust is zero (at least for the 3 fastest flow speeds, which did cross the zero thrust line and showed net drag). This result implies that faster swimming would have a lower Strouhal number (0.2-0.25) and slower swimming would have a higher Strouhal number $(0.3-0.35)$.

This nondimensionalization also allows for comparison against other data in the literature. Two of the most relevant studies of robotic pectoral fin flapping for a batoid ray are by Clark et al. [41], who tested a fin with low amplitude undulatory kinematics, and Moored et al. [40], who tested a fin with large amplitude oscillatory flapping, but with no undulation (passive or active). Both previous studies exhibit the same trend of monotonic increase of $C_{T}$ with $S t$. Measurements of $C_{T}$ reported here are similar in magnitude to those found by Moored: for example, Moored reports 


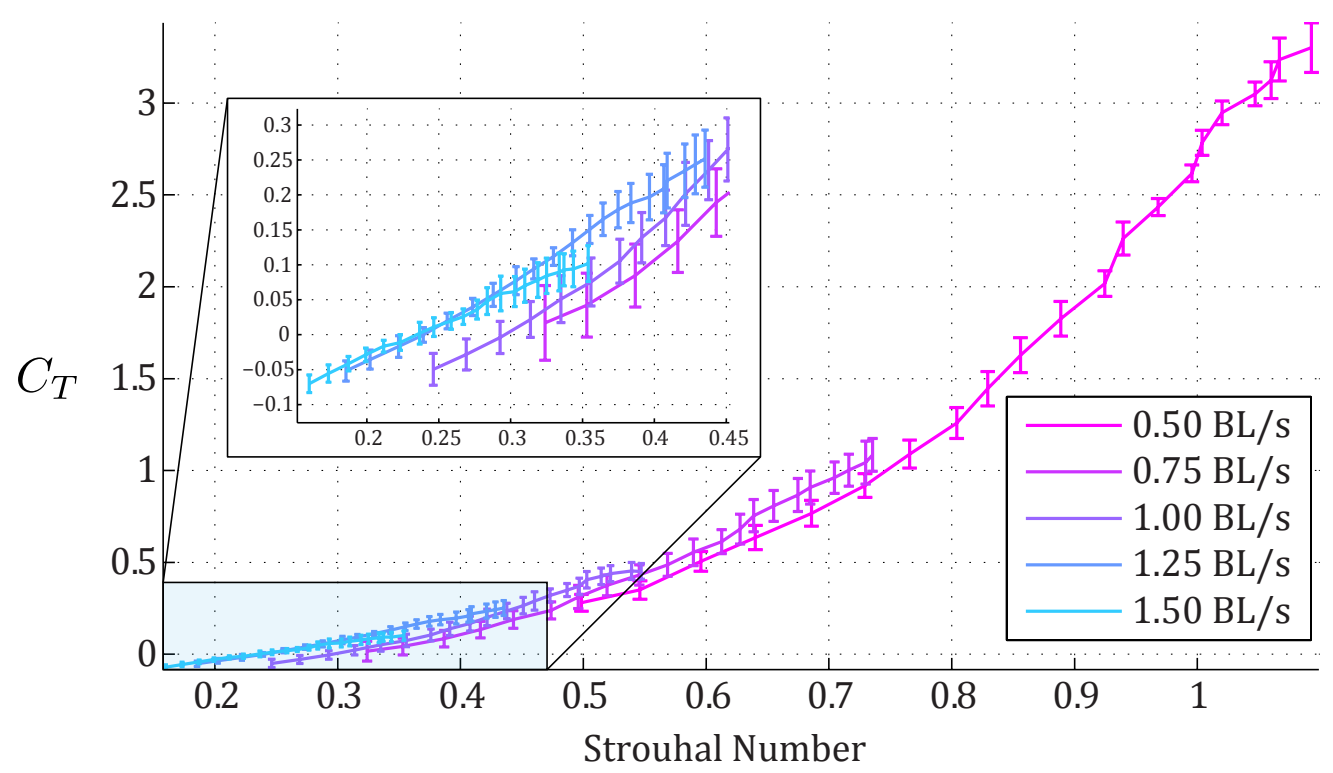

Figure 5.18: Coefficient of thrust over a range of flow speeds. Error bars represent the standard deviation from 5 tests.

$C_{T} \approx 0.2-0.5$ (depending on flapping mode) for $S t=0.4$, whereas the results here show $C_{T} \approx 0.1-0.2$ for $S t=0.4$. So it appears this tensegrity-based fin with passive undulation actually produces slightly less thrust compared to a similar fin with no undulation. In contrast, Clark reports $C_{T} \approx 1.75-3.0$ for $S t=0.4$, which is an order of magnitude more thrust compared to the tensegrity fin. The power input required to produce this thrust must be taken into consideration, as is covered next by propulsive efficiency measurements, but it seems from this result that an actively produced undulatory wave is important for creating thrust.

Taking the ratio of thrust produced to net power input to the fluid (see equation 5.3), the resulting propulsive efficiency is shown in figure 5.19. The results show a general trend over a fairly wide range (factor of 3) of imposed flow speeds: as Strouhal number is increased the efficiency rises sharply from zero (transitioning from net drag to net thrust), comes to a peak value, then decreases gradually. While this is the general trend, there are differences between the tests performed at different flow speeds. For the lowest flow speed $(0.5 \mathrm{BL} / \mathrm{s})$, the Strouhal number range is such that net drag is never reached, there is no distinct peak in efficiency, and instead 


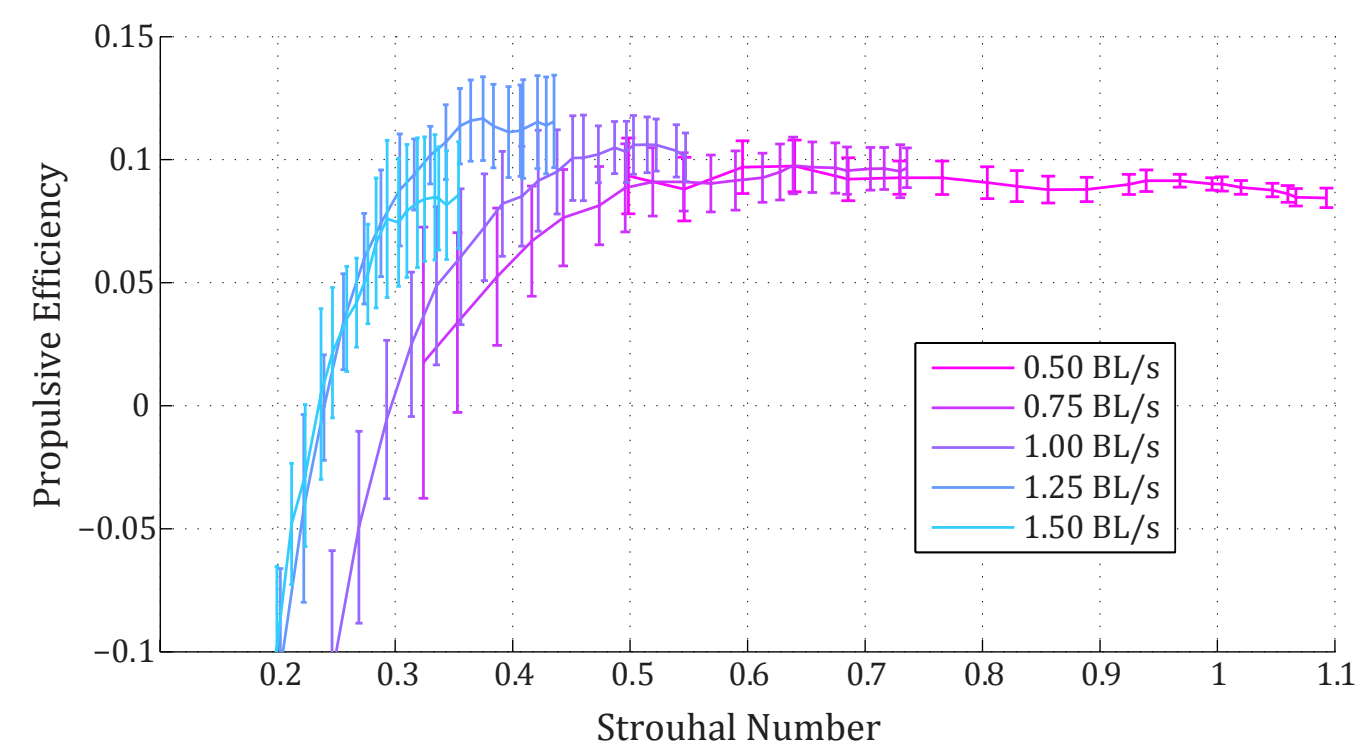

Figure 5.19: Efficiency measurements for different flow speeds, plotted against Strouhal number. Peak efficiency is about $12 \%$. Error bars represent the propagated standard deviations from 5 tests.

efficiency is relatively constant. As flow speed increases, Strouhal number is reduced for the parameter set, net thrust is reduced and peaks in efficiency become apparent. For the two cases with clear peaks in efficiency there appears to be a slight scaling in the maximum efficiency: $\eta_{\max }=11 \%$ for $U=1.0 \mathrm{BL} / \mathrm{s}$ and $\eta_{\max }=12 \%$ for $U=1.25 \mathrm{BL} / \mathrm{s}$, although these values are both within the same range of error. The highest flow speed did not exhibit a clear peak (did not reach a point at which efficiency decreased with increasing Strouhal number).

Two items are notable about these efficiency results. First, the overall efficiency is rather low. The highest peak in efficiency, $\eta_{\max }=12 \%$, occurred at $S t=0.37$ Comparing to the same two studies cited above for the thrust results [40, 41], Moored et al. found a peak efficiency of $22 \%$ and Clark et al. report a peak efficiency of $54 \%$. So the efficiency of this fin with a passive chord-wise undulatory component is actually somewhat lower than the fin from Moored et al., which was similar, but with no undulatory component. This is counter to expectations, since Moored et al. hypothesized that adding an undulatory component might be one way of improving 
efficiency. Meanwhile, the fin from Clark et al. exhibits significantly higher efficiency, but not by the same margin as $C_{T}$ results, because power input to the fluid was higher as well. As was indicated by the thrust data, these results imply that an actively produced undulatory component is superior to a passively produced one in terms of propulsive efficiency.

Second, both of the other studies show distinct peaks in efficiency occurring around $S t=0.2-0.3$, and efficiency drops off significantly for higher Strouhal numbers. The tensegrity fin in this study does not demonstrate such a distinct peak in efficiency, but instead the efficiency gradually declines across a broad range of Strouhal numbers from 0.5 to 1.1. While the maximum efficiency of the fin is rather low, it is somewhat unusual that there is not such a prominent peak in efficiency and instead a broad plateau of relatively constant efficiency. This effect is attributed to the passive compliance of the fin, which is not present in either of the other two studies.

Another difference to note about this study compared to the other two is flow velocity: The tests by Moored et al. were conducted at $U=0.48 \mathrm{BL} / \mathrm{s}$ and those by Clark et al. at $U=0.46 \mathrm{BL} / \mathrm{s}$, where body length $(\mathrm{BL})$ is the root chord length in each case. Both flow velocities are comparable to the lowest flow speed demonstrated here (which is low for biological rays [54]), and to operate in the Strouhal number range 0.20.4 they have used low amplitudes (Clark et al. ) and low frequencies (Moored et al. ). Strangely though, variation of flow velocity seems to be disregarded in other relevant studies. The results here indicate the measured efficiency has some dependence on imposed flow speed. Take for example the efficiencies measured at $S t \approx 0.35$ : for $U=0.75 \mathrm{BL} / \mathrm{s}$ the efficiency is about $3 \%$, for $U=1.0 \mathrm{BL} / \mathrm{s}$ the efficiency is about $6 \%$, for $U=1.25 \mathrm{BL} / \mathrm{s}$ the efficiency is about $11 \%$, and for $U=1.5 \mathrm{BL} / \mathrm{s}$ the efficiency is about $9 \%$.

The fact that flow speed affects measured propulsive efficiency leads inevitably to the following question: how should an imposed flow velocity be chosen in order to determine the "true" efficiency of a flapping fin? The answer has two parts: first, 
it may be that there is no single "true" efficiency - in the same sense that the efficiency varies across a range of Strouhal numbers, it can also vary across a range of flow velocities; second, it depends on what conditions these tests are designed to imitate. Ultimately, the subject of interest is a free-swimming vehicle. In steady (constant velocity) free-swimming, the average thrust and drag balance out so that net thrust is zero, meaning that Froude propulsive efficiency would also be zero. So really, efficiency defined this way is only meaningful if net thrust is positive. An example where this would be the case is a traditional rigid-hulled vessel with a rotary propeller: the hull has some coefficient of drag that can be determined for a cruising velocity of interest, and the propeller can be tested to find a coefficient of thrust to match the drag at that velocity - its efficiency at that operating point is meaningful because the sources of thrust and drag can be separated.

However, for biological swimmers (especially rays, whose fins make up a very large portion of the entire body) the sources of thrust and drag cannot be so easily separated. Even though the central bodies of myliobatoid rays are mostly rigid, they oscillate during swimming [58] and therefore a coefficient of drag for the body (determined under steady conditions) is probably not a good indicator of the thrust that needs to be produced. Even if that approach were taken, it should be noted that coefficient of drag for a streamlined body is quite low, and has been measured as $C_{D}=0.029$ for the body of a cownose ray [58]. At a coefficient of thrust matching that drag7, all the efficiencies measured in this study are extremely low. The same also holds true when looking at the results from Moored and Clark: the efficiencies corresponding to such a low value of $C_{T}$ are nowhere near as high as their peak values.

This difficulty motivates the search for some other form of performance measure that does not depend on imposed flow speed and represents the performance of a freely swimming vehicle (or animal). While the constrained tests are useful for demonstrating that the fin produces thrust (and even showing how this relates to vor-

\footnotetext{
${ }^{7}$ Assuming approximately equivalent areas for the definition of each coefficient. Even if the ratio of body to fin area is varied, the conclusion is maintained.
} 
tex shedding), the use of Froude propulsive efficiency seems ill-suited for predicting performance of a free-swimming vehicle. This is not to say that the efficiency measure has no use at all though: if two fins $\mathrm{S}^{8}$ are tested under similar conditions (with matching flow velocities) and one fin has higher efficiency than the other, then clearly the one with higher efficiency is superior at producing thrust at that flow speed. However, it is proposed here that for an oscillating propulsor where the sources of thrust and drag are inseparable, the most appropriate method for conducting performance tests is at free-swimming velocity, i.e. by running unconstrained tests. Therefore the results of tests conducted under free-swimming conditions are described next.

\subsubsection{Unconstrained Tests: Speed and Economy}

Unconstrained water tunnel tests were performed at a nominal amplitude of $A / b=$ 0.57 for a range of frequencies from $0.5 \mathrm{~Hz}$ to $1.25 \mathrm{~Hz}$. It was found that at frequencies below $0.5 \mathrm{~Hz}$, the fin could not consistently produce enough thrust to overcome the friction of the linear rail (estimated to be about $0.1 \mathrm{~N}$ ).

Figure 5.20 shows the net power of these tests plotted against flapping frequency. The magnitude of net power for the free-swimming tests was not substantially different from the constrained tests, but the shape of the curve is different for frequencies above about $0.9 \mathrm{~Hz}$. Instead of continuing to increase, the power input plateaus briefly, and then climbs even more sharply for the highest frequencies. This is in contrast to the power for the constrained tests, which all started leveling off for the highest frequencies.

Nondimensionalized as the coefficient of power (figure 5.21), this result looks drastically different than for the constrained tests. The reason for such a difference is that the coefficient of power is scaled by $1 / U^{3}$. For constrained tests, the flow velocity $U$ is constant, so the shape of the power curve is unaffected. However for the unconstrained tests, the swimming velocity changes with flapping frequency (discussed

\footnotetext{
${ }^{8}$ This could be two completely different fins, or the same fin with different sets of kinematics.
} 


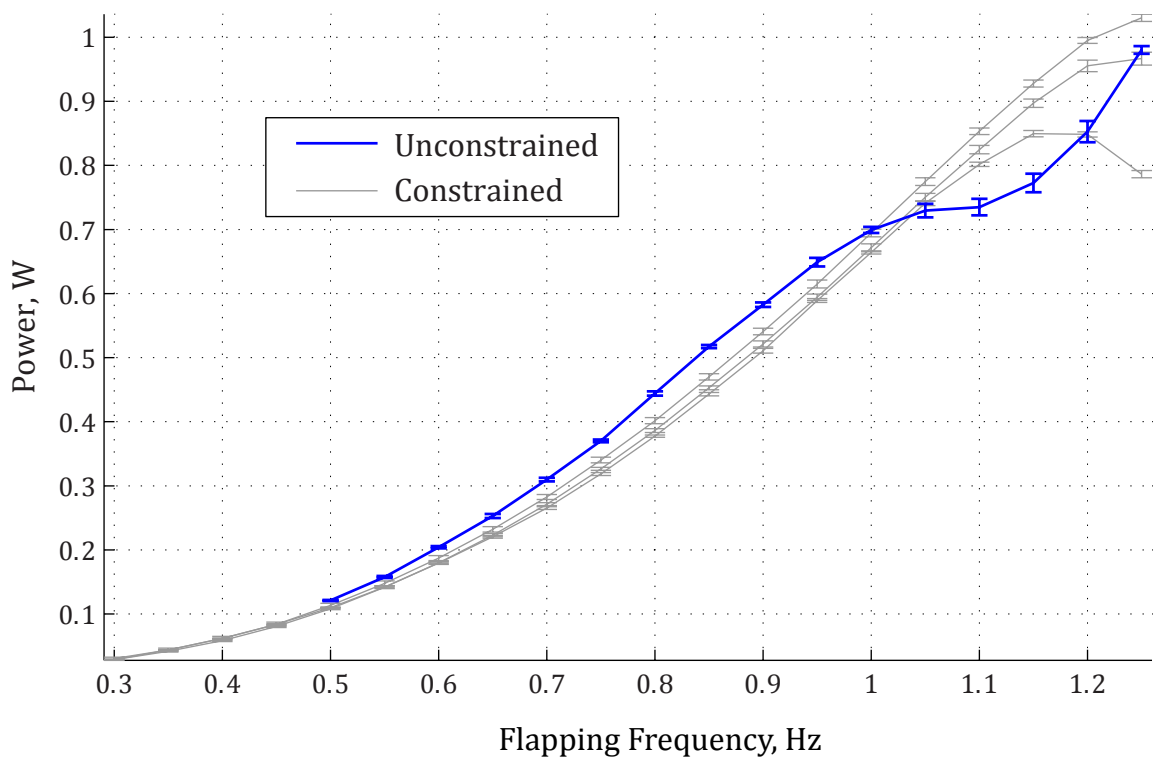

Figure 5.20: Net power for unconstrained tests plotted against flapping frequency. The magnitudes overall are similar to constrained tests, but the shape of the curve is noticeably different for higher frequencies.

next), so that a low velocity causes $C_{P}$ to become large, while a high velocity causes $C_{P}$ to become small. In this way, even though the net power to the fluid is increasing with flapping frequency, $C_{P}$ remains at a low, relatively constant level (for flapping frequencies above $0.8 \mathrm{~Hz}$ ) because the swimming velocity is increasing as well.

Figure 5.22 shows the instantaneous position of the fin along the rail for all frequencies, averaged over the 10 sets of tests. Two cycles have been removed from the beginning of each test to exclude any acceleratory portion, such that the velocity over the entire test has little variation and represents the steady swimming velocity (demonstrated by the linearity of the position plots). Note that the velocity along the rail (the slopes of these lines) does not correlate to flapping frequency because the total fin velocity is calculated as the slopes from this data plus the flow tank velocity for each test.

Figure 5.23 shows the free-swimming velocity of the fin plotted against flapping frequency. The overall trend in swimming velocity is quite similar to the net power of figure 5.20, in that it rises with frequency, then has a plateau, and rises sharply 


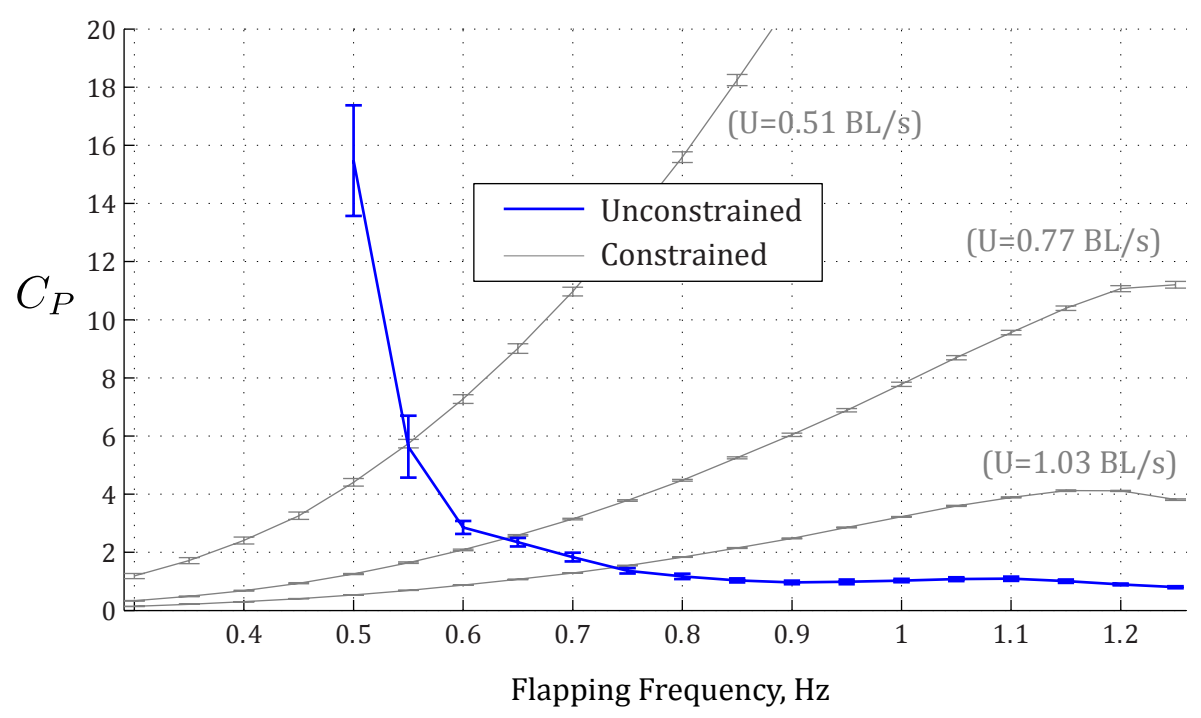

Figure 5.21: Coefficient of power versus flapping frequency for the unconstrained tests. The result is very different from constrained tests because $C_{P}$ is scaled inversely by the cube of velocity.

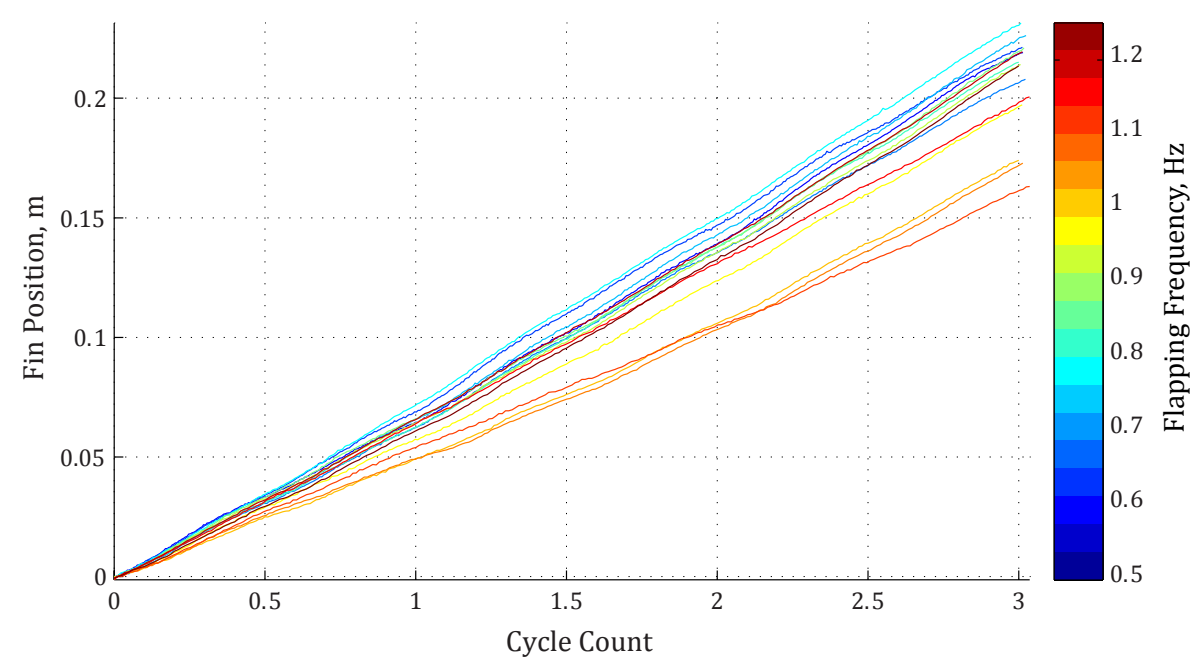

Figure 5.22: Instantaneous fin position along the rail of the experimental rig for all frequencies. The linearity of the measurements demonstrates that steady cruising was achieved. Even though total swimming velocity does correlate with flapping frequency (see figure 5.23), the slopes here do not correlate to frequency because the water tunnel velocity has not been incorporated. 


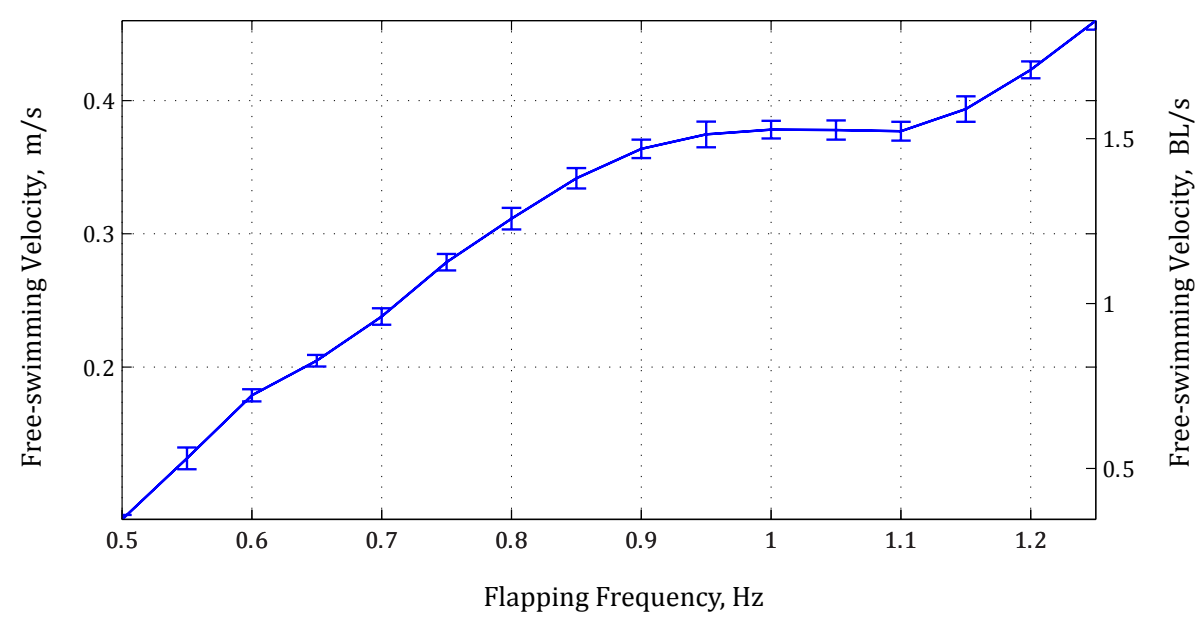

Figure 5.23: Free-swimming velocity for the unconstrained tests, shown in both $\mathrm{m} / \mathrm{s}$ (left axis) and body lengths per second (right axis).

again for the highest frequencies. The maximum swimming velocity was $1.86 \mathrm{BL} / \mathrm{s}$ $(0.46 \mathrm{~m} / \mathrm{s})$ and slowest swimming velocity was $0.35 \mathrm{BL} / \mathrm{s}(0.09 \mathrm{~m} / \mathrm{s})$. This covers the range of velocities used for constrained tests and shows that the fin is able to free-swim at significantly higher velocities than were used in constrained tests. Also, this data shows $1 \mathrm{~Hz}$ flapping produces $U \approx 1.5 \mathrm{BL} / \mathrm{s}$. In comparison, observed swimming speeds for cownose rays flapping at $1 \mathrm{~Hz}$ are between 1 and $3 \mathrm{BL} / \mathrm{s}$ [58], so the artificial fin lies directly in the middle of that range.

This velocity can also be represented as stride length, or the distance traveled per flapping cycle, as shown in figure 5.24. A maximum stride length of $1.63 \mathrm{BL}$ occurred at $f=0.9 \mathrm{~Hz}$. This is about $20 \%$ lower than reported values of stride length for cow nose rays, which are around $2 \mathrm{BL}$ [54].

Free-swimming economy is defined here as the ratio of the free-swimming velocity to net power input to the fluid. Having units of $\mathrm{m} / \mathrm{J}$, this measure directly represents the distance that can be traveled $(\mathrm{m})$ for a given amount of energy $(\mathrm{J}){ }^{9}$. Figure 5.25 shows this measure plotted against both flapping frequency and swimming velocity. Both representations have a similar shape, since swimming velocity is (roughly)

\footnotetext{
${ }^{9}$ In this way, the measure is not unlike fuel economy for automobiles, which also has units of distance per energy (miles per gallon).
} 


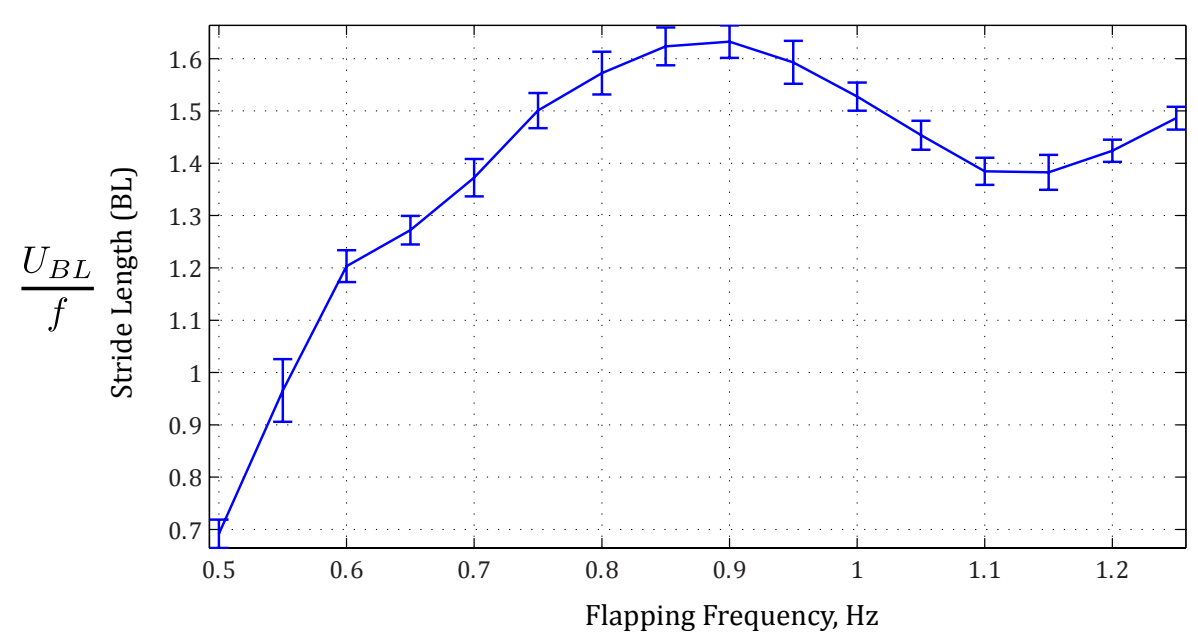

Figure 5.24: Stride length of the fin in unconstrained tests, calculated as velocity divided by frequency.

monotonically correlated to flapping frequency. This data shows a peak in economy of $\xi=0.875 \mathrm{~m} / \mathrm{J}$ at low frequency/velocity (at $0.6 \mathrm{~Hz}$ and about $0.18 \mathrm{~m} / \mathrm{s}$ ). The two very lowest frequencies/velocities have worse economy compared to the peak, and economy decreases monotonically with frequencies/velocities higher than the peak.

Why is it that the measured economy actually decreases for the two lowest flapping frequencies? The most likely explanation is that the decreased economy at the two lowest frequencies is actually an artifact of the test setup based on the inherent friction of the rail system. While the free-swimming system was made to be as low-friction as possible, it still does have friction of around $0.1 \mathrm{~N}$, which represents a finite, nonvelocity-dependent drag on the fin. Notice that the net thrust in the constrained experiments (see figure 5.17) just reaches $0.1 \mathrm{~N}$ (equal to the rail friction) at $0.6 \mathrm{~Hz}$ and $0.5 \mathrm{BL} / \mathrm{s}$. In real free swimming (not attached to any rail), drag only depends on flow around the swimmer and its magnitude should be velocity dependent, so as swimming speed slows, the drag that must be overcome by thrust decreases. Here, the friction of the rail resists very slow swimming speeds, which most likely is artificially lowering the economy measurement.

Assuming the peak in economy is an artifact of the test setup, the results show 

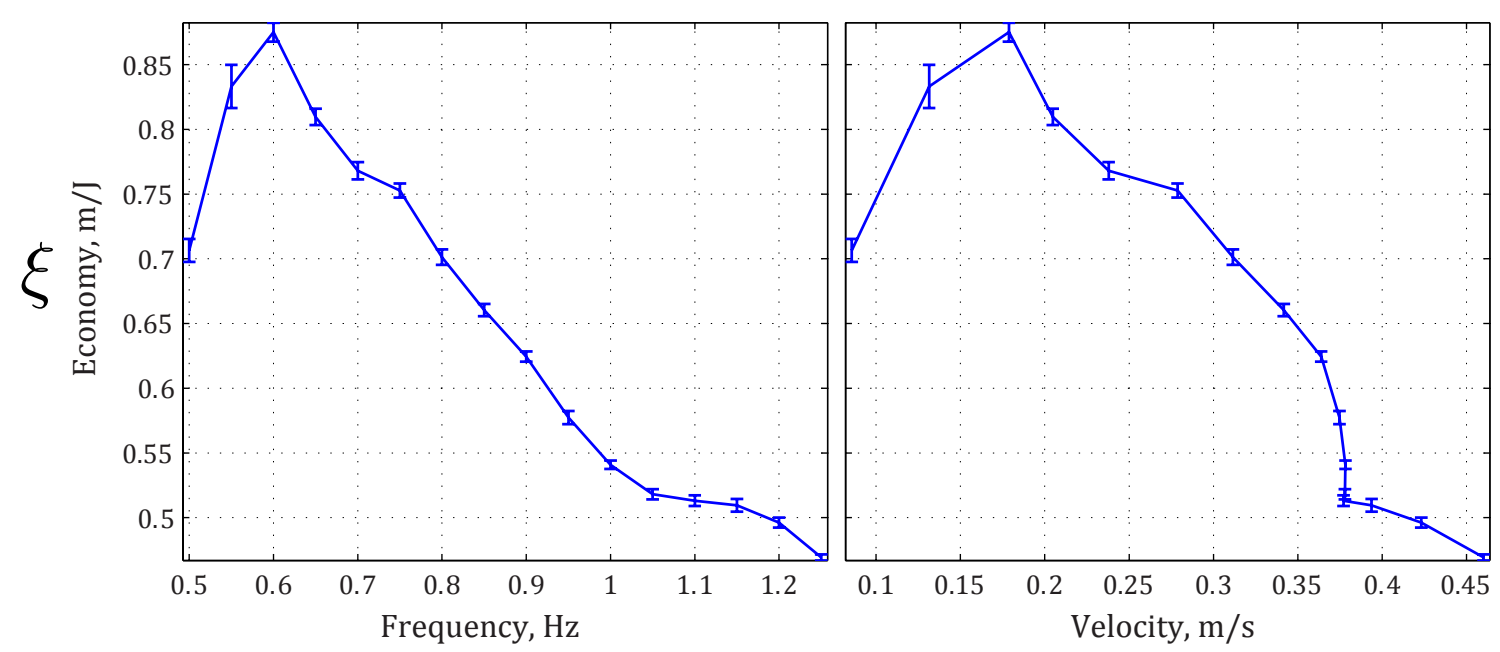

Figure 5.25: Free-swimming economy of the fin, plotted against both flapping frequency (left) and velocity (right).

that swimming as slow as possible is most economical if the goal is to minimize energy usage for a given distance traveled (without concern for the time it takes to the travel that distance). This is certainly not the only possible measure of free-swimming performance and other measures could change the conclusion about a slow optimal operating point (for instance if low velocity is penalized by some cost function). One drawback of this economy measure is that there is no immediate sense of what constitutes the best possible economy. Propulsive efficiency makes clear that $100 \%$ is the best theoretical value, and likewise that $12 \%$ is a relatively poor efficiency. However there is no clear upper limit to economy as defined here.

Also, economy measurements are extremely scarce in the literature, which makes further comparisons difficult. For example, Lauder [128] studied the thrust production of a biorobotic pectoral fin (modeled after a sunfish) and reported free-swimming velocities, but not the corresponding power measurements. Moored et al. [40] report the energy economy of an undulatory batoid fin (the same fin described in [41]), but economy is defined slightly differently, using total power of the fin not net power to the fluid, making direct comparisons difficult. Economy of the fin in this study can be calculated using total power, but that does not guarantee the results would be 
comparable, since the power to actuate each fin (in the absence of fluid loading) as a function of frequency may not be similar. When economy of the fin in this study is calculated with total power, the results are very similar to figure 5.25, just scaled downward.

Interestingly though, the results from [40] indicate the opposite trend from what is shown here: that energy economy (using total power) generally increases with frequency. The qualitative difference in the economy-frequency relationship most likely comes from the difference in actuation mechanisms, which have dissimilar mechanical efficiencies as a function of frequency 10 . Also, a major difference is that the power in [40] is total electrical power, whereas the total power in this study is mechanical, not considering inefficiencies of the servomotor. In an attempt to make a comparison, the maximum economy reported in [40] is about ${ }^{11} 0.18 \mathrm{BL} / \mathrm{J}$, while the maximum economy (based on total mechanical power) for the tensegrity fin in this study is 2.3 BL/J. However, this does not consider inefficiency of the servomotor - even if we assume the servomotor is only $10 \%$ efficient, this gives an economy of $0.23 \mathrm{BL} / \mathrm{J}$, which is larger than, but comparable to that reported in [40. Moreover, there does not appear to be a standard method of measuring the free-swimming performance of flapping underwater propulsors, which motivates the establishment of some quantity which can be applied to a variety of swimming devices. It is proposed here that a free-swimming economy based on net power to the fluid (as for Froude propulsive efficiency) is a meaningful measure that could be applied across a wide variety of swimming devices.

Another commonly cited metric related to swimming performance is Strouhal number and Taylor 64 notes that a large variety of swimmers (and even flyers) operate within the range of $S t=0.2-0.4$ in order to optimize propulsive efficiency. The Strouhal number for these free-swimming tests is shown in figure 5.26. Indeed,

\footnotetext{
${ }^{10}$ Based on personal correspondence with the authors of [40]. Mechanical efficiency of that fin is more fully described in 41

${ }^{11}$ Body-lengths per Joule, with body length reported as the root chord, the same as in this study.
} 


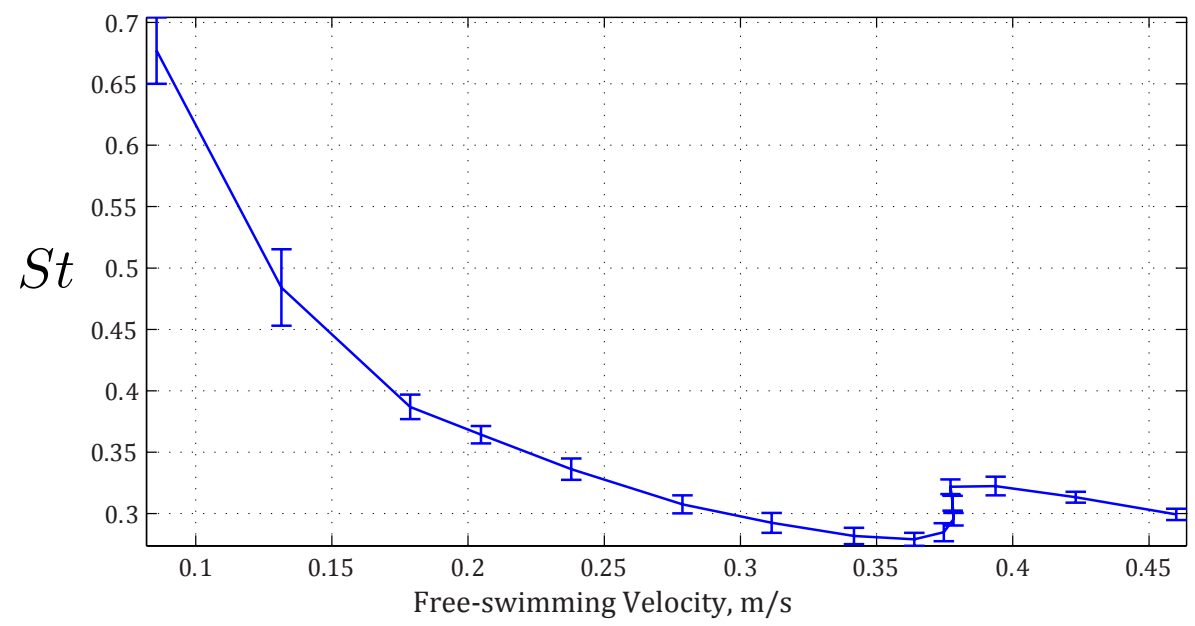

Figure 5.26: Strouhal number for unconstrained tests, showing that for most parameters, the fin operates within $S t=0.25-0.35$.

the majority of steady swimming velocities from these unconstrained tests fall right within the range mentioned above, with data points at higher velocities centered around $S t=0.3$. The two points at lowest velocities (and lowest flapping frequencies) are the only ones with Strouhal numbers outside this range, and they correspond to the same two points with lower economy, lower stride length and high $C_{P}$. All of these shifts are consistent with a low velocity compared to the other parameters of swimming, again indicating the fin may have been encumbered by friction of the linear rail at such low flapping frequencies.

Figure 5.27 shows an interesting result of the economy measurement when compared to the relative invariance of Strouhal number for most cruising speeds: for a fairly narrow range of Strouhal numbers (from about 0.28 to 0.32 ) there is a significant variation in the measured swimming economy. The highest economy in this range is $60 \%$ larger than the lowest. This is unexpected, since a variety of previous studies (as mentioned above) correlate specific Strouhal numbers uniquely with high performance, as measured by propulsive efficiency. However when using a free-swimming measure, such as the economy defined here, it appears that Strouhal number is not a unique indicator of free-swimming performance, since swimming at nearly the same Strouhal number can have both the lowest and one of the higher (85\% of maximum 


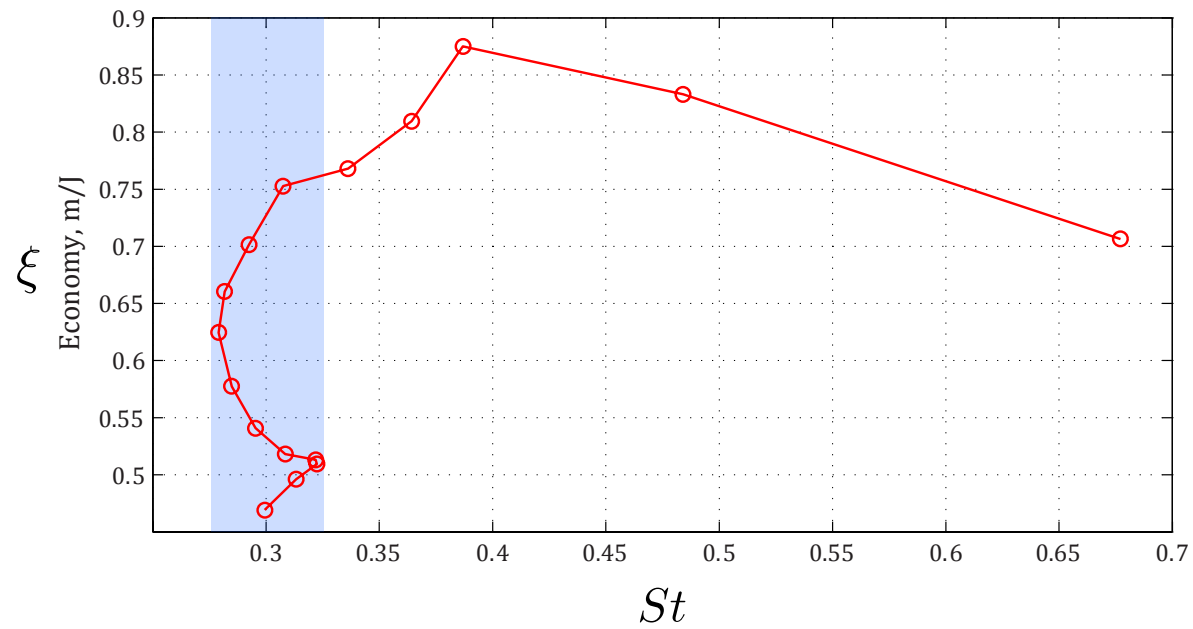

Figure 5.27: When free-swimming economy is plotted against Strouhal number, it is clear that $S t$ does not have a one-to-one correlation with this performance measure.

$\xi)$ values of free-swimming economy.

In addition to steady (constant velocity) free-swimming tests, the robotic fin was tested under acceleratory conditions to examine burst speeds for the same amplitude and range of frequencies as the tests above. The tests were conducted with no flow through the water tunnel, so that the fin accelerated from complete stop in still water. Most tests were constrained by the length of the rail to only 2 full cycles, especially at higher frequencies. Figure 5.28 shows the fin velocities after 1 and 2 cycles compared to the steady velocities discussed previously. This illustrates that flapping at $1 \mathrm{~Hz}$, the fin can reach a speed of $1 \mathrm{BL} / \mathrm{s}$ in less than 2 flapping cycles. Averaged across all frequencies, after only one flapping cycle the fin reached $40 \%$ of the steady cruising velocity, and after two cycles it reached $64 \%$ of steady cruising velocity. Overall this demonstrates the artificial fin is capable of significant accelerations, which could be a desirable trait for an underwater vehicle.

\subsubsection{Unconstrained Tests: Amplitude Variation}

All tests discussed up to this point have been conducted at a constant amplitude of $A / b=0.57$. To investigate what role amplitude modulation might play in the 


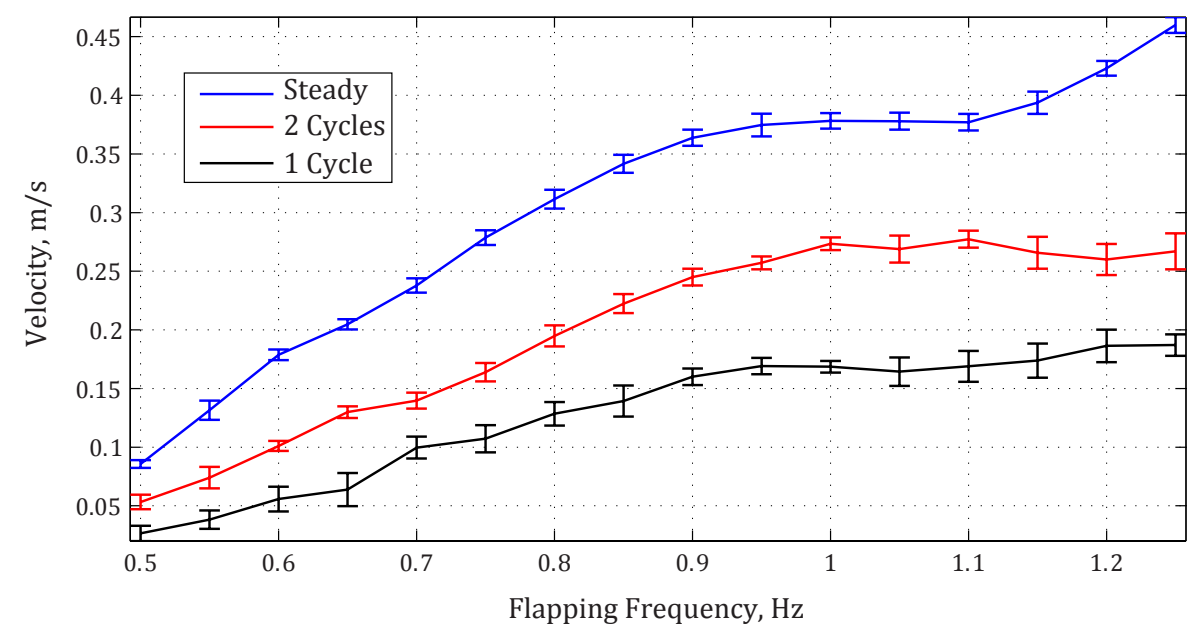

Figure 5.28: Velocity of the fin after only one and two flapping cycles (accelerated from rest in still water) compared to steady cruising velocity with the same parameters.

free-swimming performance of this fin, unconstrained tests at steady velocities were conducted for two other amplitudes: $A / b=0.42$ and $A / b=0.71$. The fin did not reliably propel itself forward at a steady velocity for the lowest amplitude until a flapping frequency of $0.9 \mathrm{~Hz}$. Figure 5.29 shows the resulting velocities for each amplitude. For each frequency, higher amplitude corresponds to higher free-swimming velocity. The largest amplitude at the highest frequency is capable of swimming at $0.61 \mathrm{~m} / \mathrm{s}$, which corresponds to $2.5 \mathrm{BL} / \mathrm{s}$ (near the upper limit of $3 \mathrm{BL} / \mathrm{s}$ recorded for cownose rays). The smallest amplitude does not swim very fast and plateaus to a fairly constant velocity of $0.25 \mathrm{~m} / \mathrm{s}$ for the highest frequencies tested. This is an interesting trend because for both of the higher amplitudes, their velocity continues to increase at the highest frequencies.

Examining the net power to swim in each case (figure 5.30), it is clear that substantially more power is required to accomplish these high velocities at high amplitude and frequency. This result also demonstrates that there is a fairly continuous power curve for all frequencies and amplitudes that correlates to swimming velocity.

The Strouhal numbers for all three amplitudes is shown in figure 5.31. The lowest amplitude tests have a somewhat higher value of $S t$ for the same velocities as the 


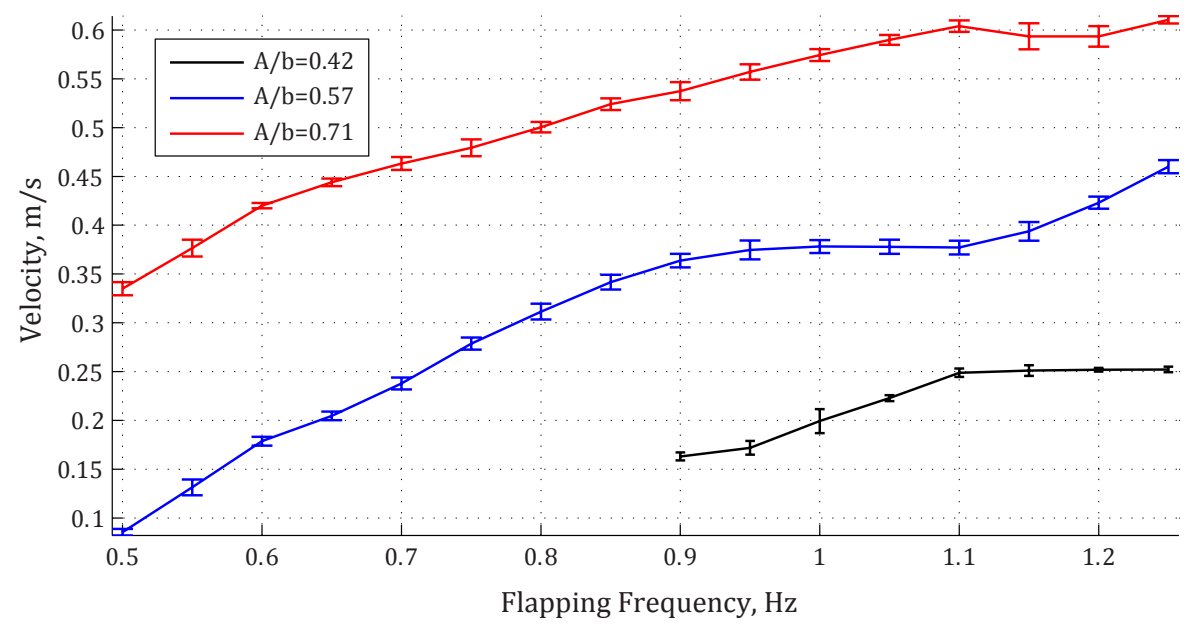

Figure 5.29: Steady cruising velocity of the fin using three different amplitudes.

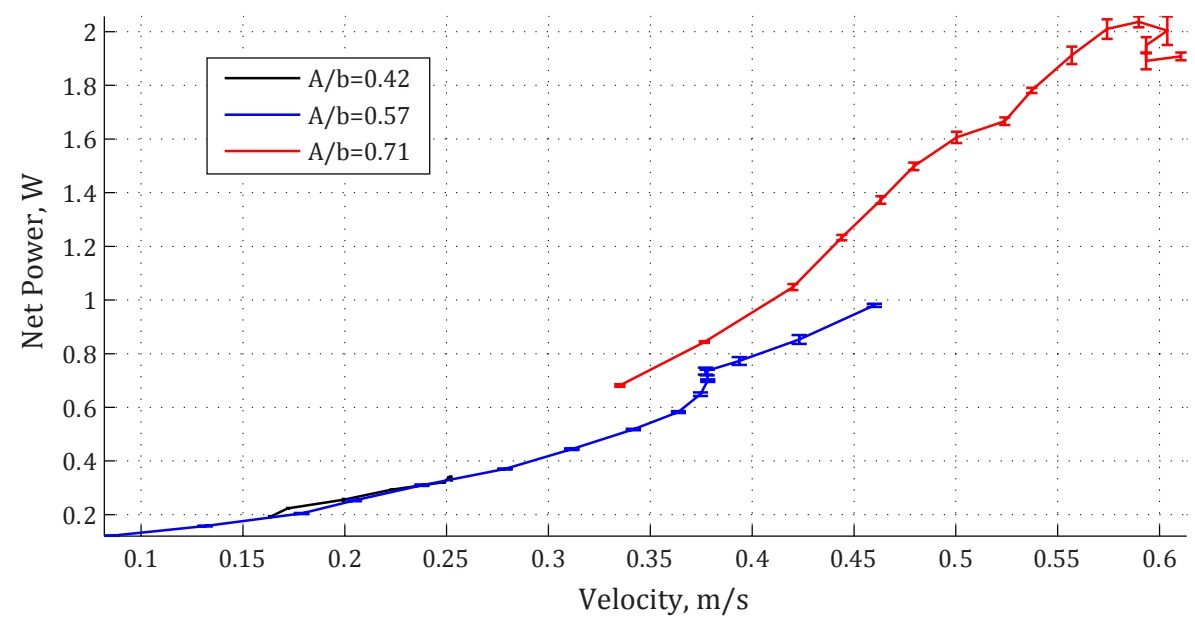

Figure 5.30: Net power input to the fluid using three different amplitudes $(A / b=0.42$ closely overlaps with $A / b=0.57$. 


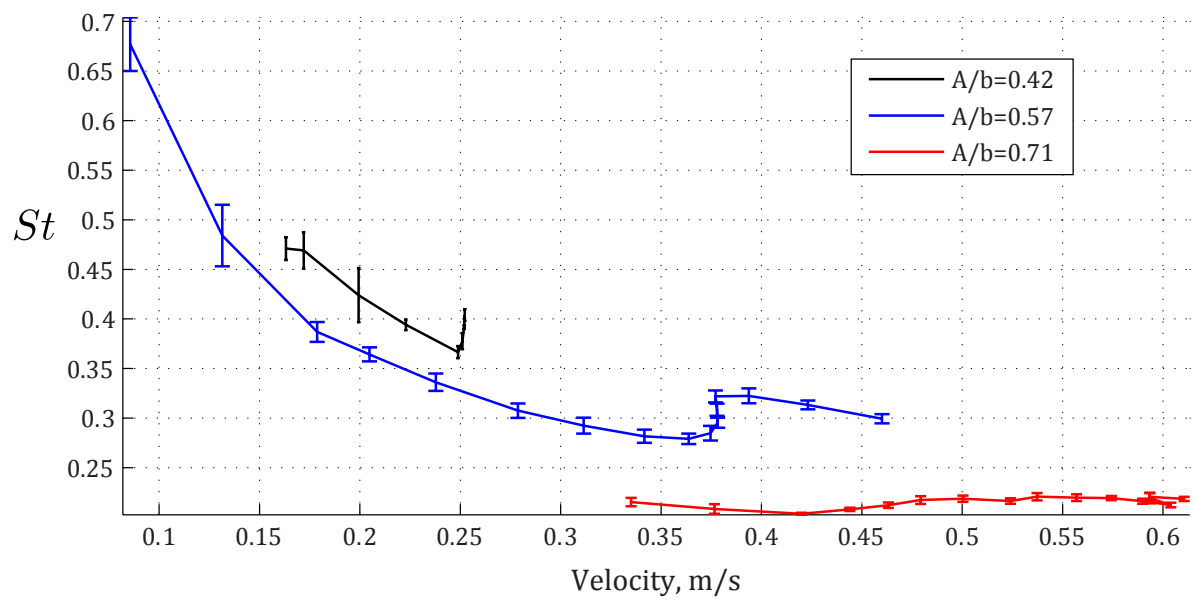

Figure 5.31: Strouhal number in free swimming using three different flapping amplitudes.

intermediate amplitude. This is because those same velocities were reached at much higher flapping frequencies. Meanwhile, the largest amplitude flapping has a relatively constant and low Strouhal number across all swimming velocities (average of $S t=$ $0.22)$.

The free-swimming economy for these other two amplitudes (see figure 5.32 follows a pattern similar to that seen for the intermediate amplitude. The lowest amplitude has similar economy values to the intermediate amplitude, and only swims at low velocities. The highest amplitude follows the trend of decreasing economy with increasing velocity. This shows that while much higher swimming velocities can be reached, they come at the price of reduced economy. In terms of operating an underwater vehicle equipped with fins like this one, this economy curve provides valuable guidance specific to the goals at hand: if least energy expenditure over a distance is desired, swimming at a low velocity is preferable; but if covering a given distance as fast as possible is desired, it can be accomplished, just at a cost of high energy expenditure. 


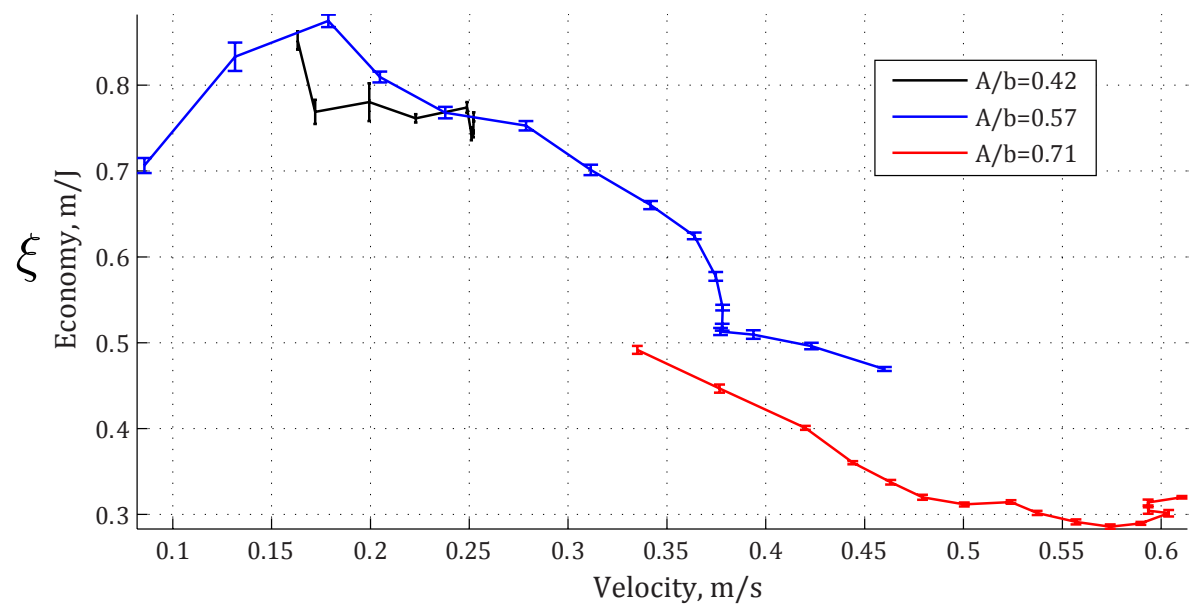

Figure 5.32: Free-swimming economy of the fin for three different flapping amplitudes.

\subsubsection{Unconstrained Tests: Skin Stiffness}

In order to investigate what role the skin material's passive compliance might play in swimming performance, a second skin was fabricated using the same mold and process as the first skin. The first skin was intended to be as soft and flexible as possible in order to maximize passive deformations and so the second skin was intended to be much stiffer, in order to minimize passive deformations, especially in the trailing edge portion of the fin. The stiffer material is a product called Dragon Skin 10, also from Smooth-On Inc., and is also a two-part liquid silicone that cures at room temperature. It is rated for a Shore hardness of 10A. To determine the stiffness of both skin materials, 6 "dog bone" samples were made with each silicone mixture and then subjected to tensile testing according to ASTM standard D 638 [129] using an Instron 5848 MicroTester. The resulting elastic moduli for the two skins were 13 $\mathrm{kPa}$ and $167 \mathrm{kPa}$ - more than an order of magnitude difference.

This second, stiffer skin underwent unconstrained water tunnel tests similar to the first, softer skin. The amplitude was similar to the intermediate amplitude of the first 


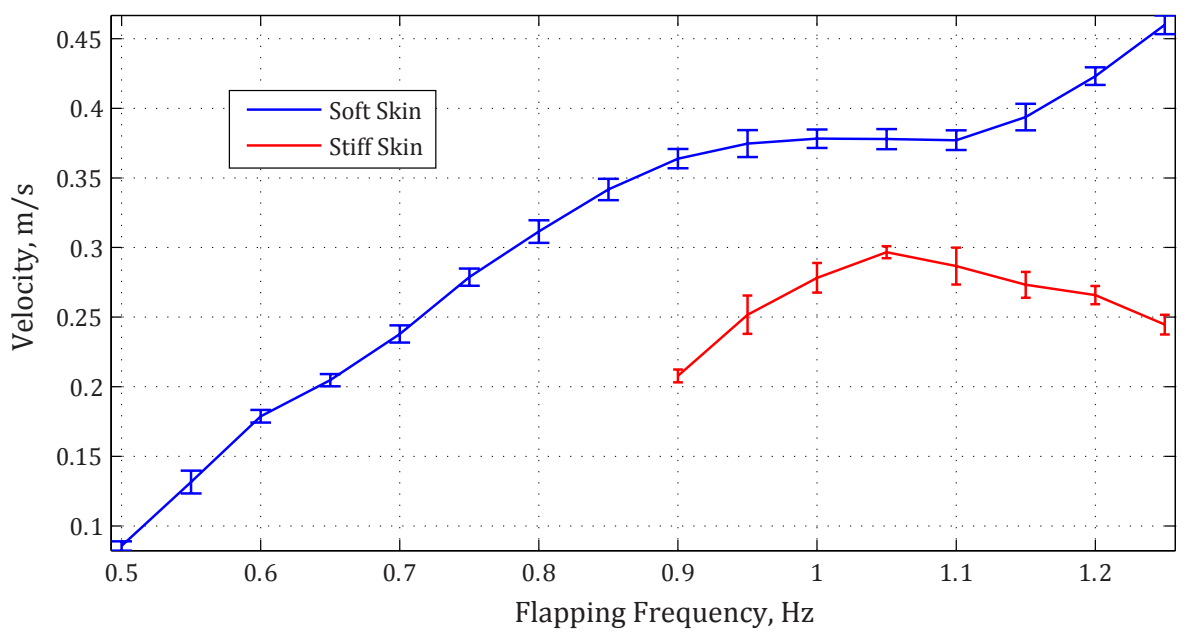

Figure 5.33: Velocity of the two different skins compared. For all frequencies, the soft skin swims faster than the stiff skin.

skin 12 , with $A / b=0.52$. The same frequency range was tested, but it was found that the stiffer fin did not reliably swim at a constant upstream velocity for frequencies lower than $0.9 \mathrm{~Hz}$, which is a similar result to the low amplitude flapping of the first skin. Figure 5.33 shows the resulting velocities for this stiff skin compared to the original soft skin. Velocities were lower at every flapping frequency and interestingly the velocity actually decreased at higher frequencies.

Figure 5.34 shows economy for the stiff skin plotted against free-swimming velocity. While this stiffer skin is capable of swimming within the velocity range of the softer skin, it does so at significantly lower economy. This is attributed to the fact that the stiff skin swims at velocities comparable to the soft skin only by flapping at higher frequencies, which are correlated to higher power input to the fluid, and so the economy is lower. These results demonstrate that flexibility can play an important role in the free-swimming performance of a flapping fin, a finding that has been shown in other studies as well [15].

To examine the amount of passive fin deformation present, photos of each skin

\footnotetext{
${ }^{12}$ Due to the high stiffness of the skin, a larger servomotor rotation amplitude was required to produce an approximately equivalent tip amplitude. While the resulting amplitude is slightly lower for the stiff skin, that does not fully account for the velocity difference (see figure 5.29) and lower amplitude does not correlate with lower economy (compare figure 5.32 with figure 5.34 )
} 


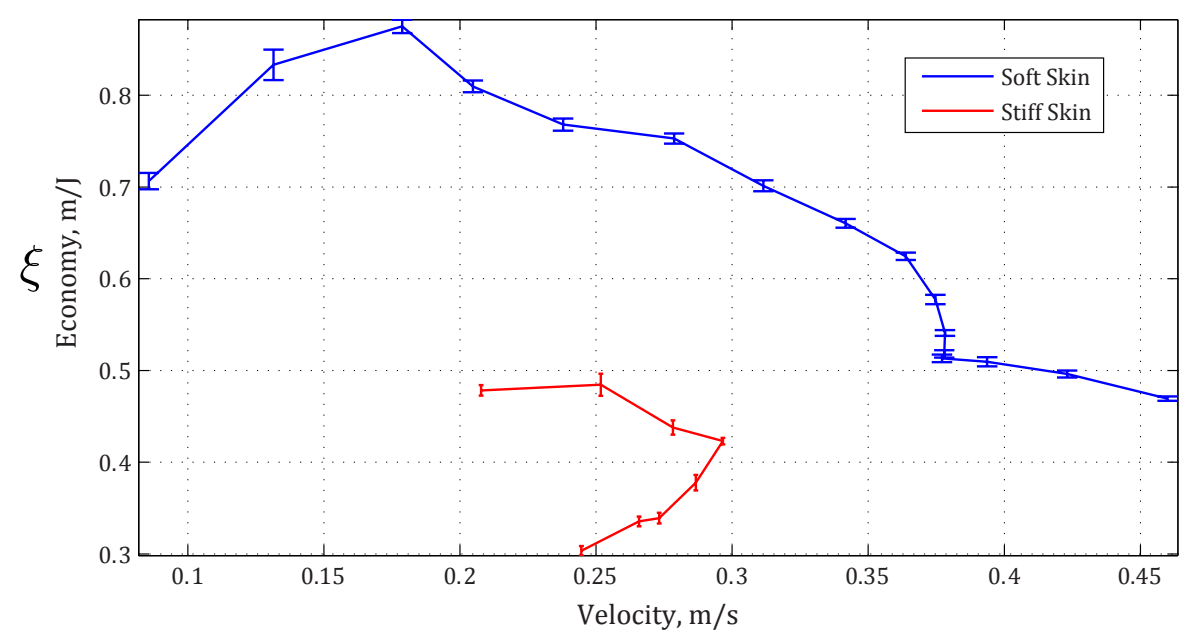

Figure 5.34: Free-swimming economy of the two different skins, showing the stiff skin has poor performance.

were taken as the fin passed through the neutral plane (triggered externally by the LabVIEW control program) at various frequencies in the water tunnel. Markers were placed at the mid-span location along both the leading edge and trailing edge, so that an angle formed by the two points could be measured. Figure 5.35 shows the results for both skins. Clearly the soft skin deforms much more under hydrodynamic loading: undulation can be seen along the soft skin that generally increases with flapping frequency, with a maximum angle from the leading edge to trailing edge of just over $7^{\circ}$; meanwhile, the stiff skin hardly deforms by any noticeable amount for any frequency (measured angle is $1^{\circ}$ at most), making it essentially chord-wise rigid. While the magnitude of this angle on the soft skin is not very large, based on the velocity and economy results it seems that this passive deformation is an important factor in the performance difference measured between the two skins. Additionally, the very tip of the soft skin lags behind fin motion and the tip of the stiff skin does not show noticeable lag, so it is possible this has some effect on performance as well. 


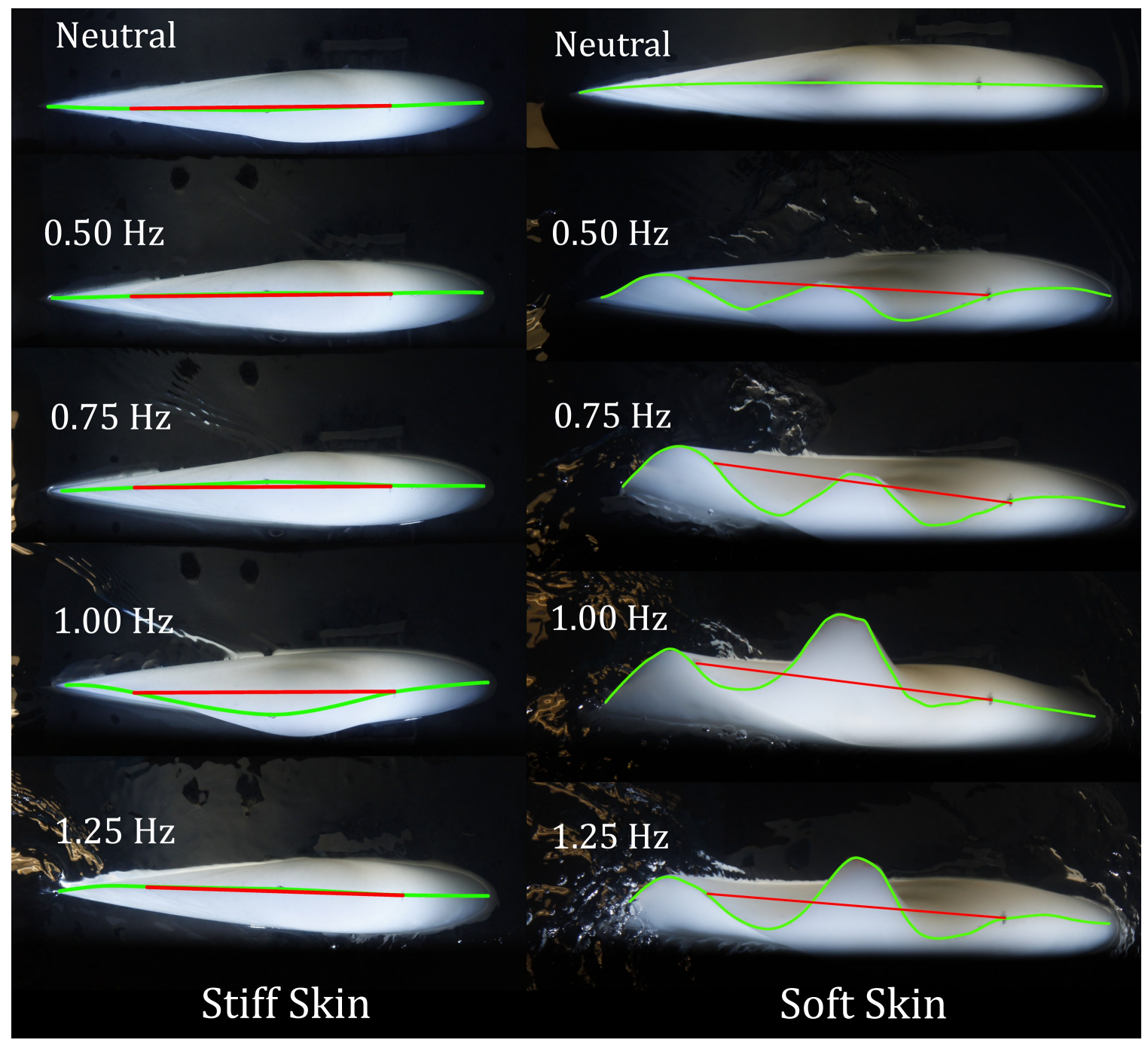

Figure 5.35: Passive deformation of both the stiff skin (left) and soft skin (right) as they pass through the neutral plane during flapping (moving downwards). The centerline along the leading edges and trailing edges have been outlined in green. The red line indicates the angle between the leading edge and trailing edge at the mid-span. 


\subsubsection{Chapter Summary}

In summary, this chapter has described the experimental methods and major results for two types of water tunnel tests on the tensegrity-based robotic pectoral fin. An experimental rig was developed to measure both the thrust produced by the fin in constrained tests and the free-swimming velocity of unconstrained tests while the fin itself is used to measure power input the fluid. Both types of tests were conducted by varying flapping frequency over a range larger than that seen in biology. It was seen that in constrained tests, both power and thrust scale with frequency. As thrust increases, the vortex shedding off the fin actually changes structure, as seen in both instantaneous thrust measurements and qualitatively in wake visualizations. The efficiency of the fin was found to be low overall, with $\eta_{F}=16 \%$ at best. Based on the assumption that biological rays do maximize propulsive efficiency to very high levels, the results suggest that the kinematics of this particular fin may be insufficient to reproduce biological performance, motivating the development of increased kinematic complexity. It was found that Froude propulsive efficiency scales based on the imposed flow velocity. It is suggested that this particular measure may not be representative of free-swimming performance if efficiencies are measured at such high levels of net thrust.

In unconstrained tests, both power and free-swimming velocity scale with flapping frequency. The artificial fin was demonstrated to be capable of swimming at speeds comparable to biological rays. A measure of free-swimming economy as the ratio of velocity to power is established, and results show this metric is generally inversely related to swimming velocity. Whether or not a true peak in economy exists is unclear, but it is shown that there is a trade-off between velocity and energy expenditure for this type of flapping propulsion. Under free-swimming conditions, this fin operates at a Strouhal number within $0.2-0.4$ (for most parameters), which is identified by many other investigators as optimal for flapping propulsion. However, based on the economy measurement established here, Strouhal number does not appear to be a unique 
indicator of swimming performance. The fin also displayed impressive accelerations, reaching on average two-thirds of steady cruising speed within just two flapping cycles. Flapping amplitude was shown to be strongly correlated with swimming velocity, but at the price of generally lower economy for the higher velocities associated with large amplitudes. Finally, it was shown that flexibility of the artificial skin seems to be important for swimming performance. Overall this study demonstrated the feasibility of using tensegrity as the basis for an artificial pectoral fin, and that this type of fin shows promise for use in underwater vehicles. There are many ways of quantifying the performance of such a fin and while several have been shown here, suggestions for further work relating to this fin are discussed in the next chapter. 


\section{Chapter 6}

\section{Conclusions}

A new generation of biologically-inspired underwater vehicles with flapping, flexible propulsors shows promise for outperforming conventional underwater vehicles that use rigid hulls and rotary propellers. Manta rays appear to be an excellent model for an efficient, high-endurance, maneuverable, and stealthy underwater vehicle. Like their biological counterparts, these vehicles would use large fins with complex deformations for both thrust and maneuvering. However, progress towards this ambitious objective requires further work in many areas, including (but not limited to): biomechanics, to better understand how and why biological rays swim the way they do; unsteady hydrodynamics, to unlock the foundations of efficient propulsion produced by flapping surfaces that create and control vortices; and advanced robotic structures, to combine the findings from biomechanics and hydrodynamics into propulsors and vehicles that can reproduce or even exceed biological performance.

Towards the goal of understanding and reproducing batoid ray propulsion, the work described in this dissertation has had several specific aims: to verify the mechanics of cable-clustered planar tensegrity beams, so that these structures can be implemented in novel fin designs; to design and construct a robotic fin that reproduces the major kinematic components of oscillatory batoid rays, using active tensegrity as the structural foundation; and to quantify the swimming performance of this robotic 
tensegrity-based fin, in order to understand how design and kinematic parameters contribute to propulsion in ray-like fins. The major contributions and findings from each area of investigation are recapitulated here.

\subsection{Summary of Contributions}

An overview of the relevant analytical mechanics for planar cross, multiple cablerouted (MC) active tensegrity beams (as developed by Moored et al. [4, 5]) has been presented. These analytical solutions provide predictions for both input actuations to achieve large bending amplitudes and the structural response to external loading, based on geometric and material properties. The analytical solutions compare well to numerical simulations, but have not previously been verified experimentally. In this study, an experimental tensegrity beam was fabricated to verify the analytical solutions, so that they can be used for the design of robotic fins. Experiments indicate excellent agreement to predictions of first-order bending stiffness, with experimentally measured stiffness differing from the analytical prediction by less than $3 \%$. The results provide confidence that similar beams can be designed according to anticipated loading scenarios. Additionally, predictions for critical slackening and optimal pretension distribution were verified, further validating the tensegrity design methods laid out.

With confidence in the predicted loading response of $\mathrm{MC}$ tensegrity beams, an actuation strategy was devised to minimize the energy input required for largeamplitude bending of active beams. An analysis of the tensegrity planar cross unit cell geometric nonlinearities indicates that the induced strain effect is non-trivial for relatively stiff structures when large-amplitude bending is desired, such as that observed in batoid ray fins. Induced strain minimization was accomplished by a rotary actuation system, using a cam drum to produce modified antagonistic actuation. A simple model for the cam drum shape was presented, along with a numerical search 
method for minimizing induced strain during actuation.

Next, a novel robotic fin was designed, consisting of an active beam embedded in the leading edge of a molded compliant polymer skin. The fin has a planform, aspect ratio, and chord-wise foil cross-sections that are similar to typical oscillatory batoid rays. The embedded active tensegrity beam was designed based on estimates from a simple fluid loading model, and uses a cam-based, modified antagonistic actuation system to minimize induced strain. The active tensegrity beam's tip amplitudes were measured as a function of input actuation, showing the beam is capable of largeamplitude bending, although the measured amplitudes were somewhat lower than predicted by the linearized analytical model. At high amplitudes (above $A / L \approx 0.4$ ), the exact analytical prediction diverges from the linear prediction (because small angle assumptions become invalid), so agreement with the linearized model is not expected in the high-amplitude regime. However, differences between the experimentally measured active beam amplitudes and the exact analytical model are less than $10 \%$ for the highest amplitudes tested, which is attributed to specific fabrication details of the beam and actuation system.

Measured amplitudes of the entire fin (tensegrity beam with artificial skin) in air also showed large span-wise bending amplitudes, demonstrating a set of kinematics similar to biological oscillatory rays. Dynamic measurements of flapping amplitudes in water showed a frequency response of the system due to fluid loading, with amplitudes generally decreasing as flapping frequency increases, but these amplitude decreases were accounted for in experiments by incorporating the actual amplitudes in Strouhal number calculations.

A hydrodynamic testing rig was designed and constructed to allow for both constrained flapping experiments (to measure thrust and efficiency) and unconstrained flapping experiments (to measure free-swimming speed and economy). The implementation of an active tensegrity beam into a biologically-inspired fin resulted in a robust propulsor design for carrying out these experiments. The fin reliably performed 
a large number of tests, on the order of $10^{5}$ flapping cycles, thus demonstrating the viability of this general design concept for use in an underwater vehicle.

Constrained water tunnel experiments showed that both net thrust produced by the fin and net power to the fluid generally increased with frequency. During these experiments, it was observed that certain sets of operating parameters produced significant surface waves, essentially exciting a resonant motion of the fluid across the width of the water tunnel. This surface wave resonance was correlated with decreases in both thrust and power, and when a plate was applied to the surface of the water to suppress these waves, thrust and power increased monotonically with flapping frequency. Comparing the propulsive efficiency when surface waves were either suppressed or not, it was found that efficiency measurements were largely unaffected by the presence of the free surface, because when the thrust and power decreased, they did so proportionally. In the Strouhal number range relevant to free swimming, the efficiency measurements were essentially identical (because surface waves were minimal in this range).

With the wave suppression plate applied, constrained tests were performed over a range over imposed flow speeds, showing that net thrust scales inversely with flow speed. When thrust and power are both nondimensionalized (as $C_{T}$ and $C_{P}$ ) and plotted against nondimensional frequency $(S t)$, the results at different flow speeds generally collapse along single curves, showing only minimal variation with flow speed (mainly for thrust). The maximum coefficient of thrust was $C_{T} \approx 3.3$ (corresponding to average thrust of $0.65 \mathrm{~N}$, taken at a flow speed of $0.5 \mathrm{BL} / \mathrm{s}$ ).

The maximum Froude propulsive efficiency for this fin was found to be rather low, at only $\eta_{F}=12 \%$ within a Strouhal number range of $0.3-0.4$. There were several interesting points arising the efficiency results. First, previous work by Moored et al. [40] suggested that adding chord-wise flexibility to a heaving oscillatory batoid fin could increase efficiency, and the fin in this study was designed to test that hypothesis, by allowing for passive chord-wise deformations. However, the efficiency 
of this fin was slightly lower (compared to the maximum of $22 \%$ from Moored et al.), showing that, based on these results, solely adding chord-wise flexibility does not appear to improve performance over a chord-wise rigid fin. Also, work by Clark et al. 41] suggested that adding span-wise curvature might improve efficiency, but the efficiency of the fin in this study (with span-wise curvature) was significantly lower (compared to the $54 \%$ by Clark et al.). The kinematics of the two fins are significantly different, in that Clark et al. actively produced an undulatory wave, whereas in this study the undulatory component of the fin was completely passive. The combination of these two results suggest that the kinematics of the single tensegrity beam fin presented here are insufficient for producing the high propulsive efficiency expected of flapping biological propulsors. Other studies of two-dimensional pitching/heaving foils indicate that high efficiencies (up to $87 \%$ [65]) can be achieved on high aspect ratio propulsors with the proper combination of kinematics, so recommendations for kinematic improvements to a tensegrity-based fin are given in section 6.2.

A second finding from the efficiency results was that a relatively broad plateau of highest efficiency was demonstrated, instead of a sharp, distinct peak as shown in other studies [4, 41]. This is attributed to the flexibility of the fin in this study, which could be accommodating more optimal vortex formation over a broader range of St, compared to the completely prescribed kinematics on the entire fins by Moored et al. 4] and Clark et al. [41. This finding indicates that flexibility could be very important in the design of flapping propulsors in order to increase their range of optimal thrust production.

A third finding from these results is that measured propulsive efficiency has some sensitivity to the imposed flow speed in these constrained tests. It was demonstrated that measured efficiency scaled with flow speed, especially in the range $0.2<S t<0.4$, making it difficult to claim there is a single value of efficiency for this fin at a given Strouhal number. Thus, it seems that for this low aspect ratio, high amplitude flapping propulsor with passive flexibility, propulsive efficiency is not uniquely determined 
by Strouhal number. This is a somewhat surprising conclusion, since many studies have emphasized the importance Strouhal number in determining optimal propulsion 63 65]. While the peak efficiency does occur within the expected optimal range of $S t$, it also varies with flow speed, thus the following question naturally arises: what is the "correct" flow speed at which to perform these types of tests? There is no clear answer from the results of this study, but it seems that performing constrained tests under an imposed flow speed to create net thrust may not be the best measure of swimming performance for a biologically-inspired oscillating propulsor. Instead, a more appropriate measure of swimming performance could come from unconstrained, free-swimming tests, where average net thrust is zero (and thus propulsive efficiency would be zero as well), and the flow speed is self-determined by the motions of the fin and the surrounding fluid.

Unconstrained tests showed that free-swimming speed is strongly correlated with both flapping frequency and amplitude. The highest free-swimming speed reached by the fin was $2.5 \mathrm{BL} / \mathrm{s}(0.61 \mathrm{~m} / \mathrm{s})$, and occurred for high values of frequency $(1.25 \mathrm{~Hz})$ and amplitude $(A / b=0.71)$. Both the speed and kinematic parameters of this fin are fairly similar to those seen in biological oscillatory rays [58]. Using the ratio of free-swimming speed to net power to calculate economy, it was apparent that the high velocities and amplitudes used to reach the fastest speeds come at the cost of low economy. The peak economy measured was $0.875 \mathrm{~m} / \mathrm{J}$, and economy was found to generally increase as swimming speed decreased. However this was not the case for the very lowest swimming speeds (corresponding to lowest flapping frequencies), where economy was lower than the peak. This was mostly likely an artifact of the experimental test rig caused by the small but finite friction along the rail. Based on results of the constrained tests, net thrust at the lowest flapping frequencies was small enough that free-swimming speed would be affected by friction of the rail. Indeed, the peak in economy corresponds to the frequency at which net thrust just exceeds the measured friction of the rail $(0.1 \mathrm{~N})$. Therefore it is expected that a completely 
free-swimming fin (not attached to a velocity-independent source of drag) would show monotonically increasing economy for decreasing swimming speed.

The implication for the design and operation of an underwater vehicle is that the cost associated with covering some distance is directly related to speed at which that distance is covered - high speeds can be attained, but at the tradeoff of low economy, and economical transport can be achieved if the time it takes to cover a given distance is not important. It is possible that some other performance metric or cost function which combines the importance of economy and travel speed could reveal an optimal operating point of intermediate speed and intermediate economy. There are very few examples in the literature of free-swimming economy measurements for flapping propulsor: ${ }^{1}$, and it is suggested that the economy measurement used here - the ratio of free-swimming speed to net power to the fluid (similar to the power used for Froude propulsive efficiency) — could be used in other studies for comparison.

Other studies [63, 64] often note that biological swimmers operate within a range of Strouhal numbers between 0.2 and 0.4 , and that doing so indicates some optimization of propulsive performance. When free-swimming, the tensegrity fin operated within that range for most parameters, but at the lowest speeds (mostly below about $0.75 \mathrm{BL} / \mathrm{s}$ ) the Strouhal number increased, up to a maximum of nearly $S t=0.7$ (however the highest Strouhal numbers correlate to low velocity and economy, and were likely impacted by friction of the linear rail). Towards higher speeds, the Strouhal number settles to a narrow band that is amplitude dependent: higher amplitudes $(A / b=0.71)$ correlate to lower Strouhal numbers $(S t \approx 0.2)$, and lower amplitudes $(A / b=0.57)$ correlate to higher Strouhal numbers $(S t \approx 0.3)$. For a fixed amplitude, an interesting result arises from the range in economy observed compared to the relative invariance of Strouhal number: a wide range of free-swimming economy values, from the lowest to one of the highest (a $60 \%$ difference), occurred for a very narrow band of Strouhal number $(0.28<S t<0.32)$. The implication is that Strouhal

\footnotetext{
${ }^{1}$ Biological studies often used cost of transport (COT), which is similar to the inverse of economy, but accounting for body mass and often using total metabolic power.
} 
number is not a unique indicator of free-swimming economy. As with the efficiency results, this is somewhat counter to expectations, considering Strouhal number is typically associated with high propulsive performance. The combination of these two findings indicates that while high performance (however that might be measured) occurs within a Strouhal number range, just operating within that range does not guarantee high performance.

In addition to the steady-state experiments, the fin was tested for acceleration performance. It was found that after only 2 flapping cycles the fin reached nearly two-thirds of its steady-state speed, averaged across all frequencies. This type of acceleration performance could be desirable for a highly maneuverable underwater vehicle.

Additionally, a stiff artificial skin (with a modulus of elasticity one order of magnitude higher than the compliant skin) was tested under free-swimming conditions. Results demonstrated that the stiff skin only self-propelled starting at higher frequencies compared to the soft skin (minimum free-swimming frequency of $0.9 \mathrm{~Hz}$ for the stiff skin versus $0.5 \mathrm{~Hz}$ for the soft skin). While the stiff skin was able to swim at speeds comparable to those of the soft skin, it only did so at much higher frequencies, and as a consequence, at much lower values of economy (about one-third lower economy at best). Optical measurements of passive fin deformation indicate the differences in performance can only be attributed to the passive deformations of the skins, since the other major kinematics (frequency and amplitude) were comparable. Swimming at a high economy over a wide range of speeds is desirable for an underwater vehicle, so these results demonstrate that passive compliance and deformations should be taken into account when designing and testing flexible propulsors.

Overall, this work has demonstrated that active tensegrity structures can be successfully implemented into a biologically-inspired propulsor that is capable of approximating the kinematics and swimming speeds of oscillatory batoid rays. Unfortunately, there is minimal quantification of the swimming performance of biological rays (with 
no direct measurements of propulsive efficiency or free-swimming economy), so other direct comparisons are difficult to make. To reproduce the complex motions and fine control seen on manta ray pectoral fins, far more work must be done in several areas, but hopefully this research has shown that significant progress can be made using relatively simple structures and methods. The potential benefits of an unmanned underwater vehicle that can meet or exceed biological manta ray capabilities are enough to motivate further research, so a few of the most obvious and promising areas of study are given next.

\subsection{Future Work}

Many fertile areas of research exist based on studying batoid ray swimming, with opportunities that build upon this work, and in areas completely unexplored here. Since this study has mainly focused on reproducing batoid kinematics and experimentally measuring the resulting performance related to those kinematics, ideas in this section will be closely related to those efforts.

In order to open up the possibility of more closely matching certain aspects of batoid kinematics, several modifications to tensegrity-based fins could be made. By using multiple actuators for a single beam, an actuation system could control the deformation of each cell of a tensegrity beam independently, such that the phase between each cell could be varied, in order to actively control various bending modes. Manta rays demonstrate non-monotonic curvature in the span-wise direction of the their fins during flapping, and while some of this motion may be passive, the influence of this kinematic component could be explored ${ }^{2}$ with relatively simple modifications to the type of fin presented here. Along this same line, the influence of passive deformations in the span-wise direction could be investigated by "under-actuating"

\footnotetext{
${ }^{2}$ The effect of a "tip lag" was explored by Moored et al. 40. It was not found to have a large influence on efficiency, but it is possible the parameter space was not large enough to see differences, and that other changes to the fin (like swept tips or chord-wise curvature) would allow for larger performance differences.
} 
the fin, either by selectively deactivating the more distal cells in the tensegrity beam, or by building several different beams of varying number of cells, so that a beam with only one or two cells (and being $1 / 3$ or $2 / 3$ the original beam length) could drive the fin. If it were uncovered that similar performance could be achieved with fewer active cells and by exploiting passive deformations in the span-wise direction, this could simplify the design and construction of fins and vehicles.

One advantage of the tensegrity approach is that the actuation results are indifferent to the source of active cable strain, so as new types of actuators (especially "artificial muscles") are developed, they can be used. Implementing novel types of actuators into the tensegrity fin could provide more silent operation for a UUV compared to conventional servomotors. Additionally, improved actuator efficiencies would be useful for extending operating ranges and times in a free-swimming vehicle. While a remote actuation strategy has proven to be effective, new types of actuators that are strong, light, and small could make embedded actuation less prohibitive, enabling more complicated structures without concern for cable routing and friction across nodes.

The design and analysis tools for much more complex tensegrity structures exist [4], but they seem prohibitively difficult to construct compared to simple beam structures. However, having fully prescribed fin kinematics coming from an underlying three-dimensional tensegrity plate structure could be advantageous compared to relying on passive fin deformations. This could be especially true if more complex active motions are necessary, such as for turning and maneuvering, compared to relatively simple straight-line swimming. Therefore it seems worthwhile to investigate the construction and actuation three-dimensional tensegrity structures for the purpose of recreating batoid kinematics.

Based on the relatively low propulsive efficiency measured using the one degree of freedom, single-beam fin in this study, it appears that trying to create the undulatory component of batoid fins completely passively is insufficient for producing a fin design 
with high propulsive efficiency. The maximum passive deformation measured on the compliant skin in this study was only about $7^{\circ}$ (angle between the leading edge to trailing edge line and the neutral plane), while Heine [58] measured twist angles on the fins of cownose rays in the range of about $10^{\circ}$ to $25^{\circ}$. It is possible that increasing the passive deformation angle up to this biological range could improve performance. For instance, if the total area of the fin is increased, but the beam width and maximum fin thickness were maintained, then the beam width would be a lower percentage of the chord length, leaving much more of the fin available for passive deformations.

There is also significant potential for investigating ways to influence the directionality of passive deformations and the effect this could have upon propulsive performance. The passive portion of the fin used in this study is composed of artificial skin made with an isotropic material (silicone elastomer), however manipulation of passive deformations could be accomplished through either material anisotropy or passive structural patterns embedded within the skin. For example, Russo [48] studied the influence of inter-radial joint pattern angles, based on observations of the fin architecture in biological rays, finding that the underlying joint patterns could have a significant influence on swimming performance by changing the undulatory component of fin kinematics. Furthermore, the stiffness of the fin in this study is constant, whereas biological swimmers are thought to actively change the stiffness of their fins through muscular contractions. Investigating ways to actively change the stiffness of artificial structures during flapping could potentially lead to increases in performance or extend the operating range over which high performance can occur.

Based on a combination of other findings and the observed kinematics of this fin, it seems that adding at least one more degree of freedom could be important for improving performance. Heine [58] measured the maximum angle of attack for most portions of cownose ray fins to be less than $20^{\circ}$. Anderson [65] found that high values of efficiency (up to 87\%) could be attained with a two-dimensional pitching/heaving foil by maintaining a nominal angle of attack between $15^{\circ}$ and $25^{\circ}$. In this study, 
the maximum angle of attack at the mid-span of the fin during free-swimming ${ }^{3}$ was more than $40^{\circ}$, which is obviously much higher than both that observed on biological rays and for optimal thrust production in other flapping foil studies. At such high angles of attack, undesirable flow conditions (such as separation, even considering the Wagner effect) that reduce the performance of the fin could occur. Therefore it is suggested that an additional degree of freedom should be added to the fin to actively control pitch angle, which would maintain a more optimal angle of attack throughout the flapping stroke. This additional degree of freedom could be accomplished with a fairly simple design modification to the current fin, solely by mounting the tensegrity beam on a rotational stage that is actively rotated by a second servomotor. Doing so would allow for the experimental study of combinations of pitch and heave (and importantly, the phase between the two) on a batoid-like fin, which is absent from the literature. It is expected that kinematic combinations could be found on such a fin that have considerably higher efficiency compared to the purely heaving fin in this study.

Creating an artificial fin that could span the undulation-oscillation continuum of batoid locomotion could prove to be a valuable tool for understanding how these two swimming modes are related, and why some ray species switch between these gaits. One way of accomplishing this while still using planar tensegrity beams is to arrange a series of beams chord-wise along the fin, with the possibility of creating a phase difference between each beam, as suggested by Moored et al. [5]. Such a fin design would allow for large-amplitude span-wise bending and also an actively controlled undulatory wave, so that kinematic modes ranging from pure heaving (extreme oscillation) to highly undulatory. One obstacle to overcome with this design is the potentially large amounts of skin strain that could be created between each beam. For

\footnotetext{
${ }^{3}$ The maximum angle of attack at the midspan can be estimated as $\alpha=\arctan (\pi S t)$ since Strouhal number is a ratio of the fin's heave velocity to the free-stream velocity. Passive deformations of the fin can be thought of as effectively reducing the actual angle of attack, but only slightly, since the maximum measured deformation angle is about $7^{\circ}$. The angle of attack is even higher towards the tip of the fin, and is much higher at some of the Strouhal numbers used in constrained tests.
} 
large amplitudes and large phase differences (high wavenumber), the change in length between each beam tip could be large, meaning a substantial amount of actuation energy would be spent on straining the skin. This situation could be circumvented by allowing only sets of parameters that permit a certain maximum amount of strain between each beam tip. Alternatively, other types of artificial skins could be developed instead of the molded compliant polymer strategy used in this study. That could be some kind of corregated/baffled membrane to accommodate the high strain, or even a skin made of thin plates allowed to slide past one another ${ }^{4}$.

Regardless of the underlying structure, variations in the planform of the fin should be investigated, to determine what effect aspect ratio, swept tips, and other features have upon swimming performance. Studying variations of planform even beyond what is extant biologically has important implications for explaining why the planform of oscillatory batoids is actually quite consistent. Other subtle changes to fin shape may have importance for performance as well. For instance, the addition of tubercles (basically bumps along the leading edge of the fin) — a feature not found in rays has recently been shown to delay stall in foils at high angle of attack [19, 119], and could lead to performance improvements.

Without changes to the fin, additional water tunnel tests could be performed with the current structure, studying several areas unexplored in this study. Many biological swimmers, including rays, demonstrate burst and coast behavior, and the implications of this on swimming economy could be tested with the same fin and experimental setup in this study. Also, a detailed study of the effects of waveform profile could be performed, in which the actuation waveform is perturbed from sinusoidal. This type of work might help uncover why some batoid rays modulate swimming speed with fin tip speed instead of flapping frequency

\footnotetext{
${ }^{4}$ Such a design is biologically-inspired, since many fish actually use a similar scheme, in which their scales (rigid plates) are allowed to slide past one another to accommodate a highly flexible body [130].

${ }^{5}$ Moored 4, 40 investigated this somewhat with a chord-wise rigid fin, and it was not found to be beneficial to efficiency, but it was hypothesized that this outcome could change for a fin which chord-wise flexibility.
} 
The current experimental setup could be altered to measure forces in other directions, most importantly laterally (in the heave direction). Doing so would give information about how much the flapping motions transfer momentum to the fluid laterally, which is unproductive for creating thrust. Measurements of this sort would help explain why the efficiency of the fin in this study was quite low - if the heaving fin is generating lateral forces that are much higher than the thrust produced, this further motives kinematic changes, such as the addition of active pitch angle control (which could act to convert lateral forces into thrust forces by simply changing the angle of the instantaneous lift vector). This idea could be incorporated experimentally rather easily, by mounting the fin to a multi-axis force/torque sensor, and mounting that sensor to the linear rail. Such a setup would allow for the measurement of forces (except thrust) and moments during free-swimming tests, which would be informative.

The results of this study indicate that flexibility seems to be important for swimming performance, but only coarse measurement of the passive fin deformations were made. Fully characterizing passive deformations of a flexible fin will be important for relating flexibility to performance. Ideally the tracking of deformations would be fully three-dimensional, which could be be accomplished with a variety of technologies, including time-resolved 3D scanners, laser range-finding techniques, and optical stereo photogrammetry. These technologies could be applied not only to artificial fins, but to biological swimmers as well, which could be immensely helpful for determining the kinematics of rays in order to establish baseline target deformations for an artificial fin! 6 . Fully resolved fin deformations (either artificial or biological) would also be of great use to researchers performing computational fluid dynamics, because it would allow for the direct comparison of performance and could help validate their computational tools.

To fully understand force production mechanisms, three-dimensional flow visu-

\footnotetext{
${ }^{6}$ One recent study has measured the 3D kinematics of undulatory batoid rays [131].
} 
alizations need to be performed. Other studies have shown that wake structure is intimately tied to thrust production [41, 63], so to more fully understand swimming performance, wake structures should be visualized. Ideally this would be quantitative, meaning the use of particle image velocimetry (PIV). This type of work would definitively link force production to 3D wake structures, and elucidate the sources of optimal swimming performance.

By studying kinematics using stiff structures with prescribed motions, the work presented here has ignored the effects of structural resonance and the potential this effect may have to increase overall propulsive efficiency. Recent work by Bliss et al. [109, 110] has shown robust entrainment of a compliant tensegrity caudal fin to optimal gaits, using advanced control schemes. Thus it is entirely possible that kinematics similar to a stiff fin could be accomplished with reduced energy expenditure by using a more compliant structure and exploiting its resonant properties. Recent work by Moored et al. [132, 133] demonstrates that coupling together the resonance of unstable wakes with structural resonance of flexible propulsors can lead to a "global" optimal efficiency of thrust production. This coupled resonance may be exploited by biological systems, and biologically-inpspired vehicles that harness both wake and structural resonances have the potential for high performance. 


\section{Bibliography}

[1] J Yuh. Design and control of autonomous underwater robots: A survey. Autonomous Robots, 8(1):7-24, 2000.

[2] Promode R Bandyopadhyay. Trends in biorobotic autonomous undersea vehicles. Oceanic Engineering, 2005.

[3] Office of Naval Research Broad Agency Announcement 0\%-036, Topic \#3: Biologically-Inspired Autonomous Sea Vehicles, 2007.

[4] Keith William Moored III. The Design of a Novel Tensegrity-Based Synthetic Pectoral Fin for Bio-Inspired Propulsion. PhD thesis, ProQuest, UMI Dissertation Publishing, September 2010.

[5] Keith William Moored III, Trevor Kemp, Nathan E Houle, and Hilary BartSmith. Analytical predictions, optimization, and design of a tensegrity-based artificial pectoral fin. International Journal of Solids \& Structures, 48:31423159, 2011.

[6] D Richard Blidberg. The Development of Autonomous Underwater Vehicles (AUV); A Brief Summary. IEEE ICRA, 2001.

[7] R Hutchinson. Jane's Submarines War Beneath the Waves: From 1776 to the Present Day. New Line Books, 2006.

[8] William Kohnen. Manned research submersibles: State of technology 2004/2005. Marine Technology Society Journal, 39(3):122-127, 2005.

[9] James Louis Tangorra, S. Naomi Davidson, Ian W Hunter, Peter G A Madden, George V Lauder, Haibo Dong, Meliha Bozkurttas, and Rajat Mittal. The Development of a Biologically Inspired Propulsor for Unmanned Underwater Vehicles. IEEE Journal of Oceanic Engineering, 32(3):533-550, July 2007.

[10] Kamran Mohseni. Pulsatile vortex generators for low-speed maneuvering of small underwater vehicles. Ocean Engineering, 33(16):2209-2223, November 2006.

[11] D T Roper, S Sharma, R Sutton, and P Culverhouse. A review of developments towards biologically inspired propulsion systems for autonomous underwater vehicles. Proceedings of the Institution of Mechanical Engineers, Part M: Journal of Engineering for the Maritime Environment, 225(2):77-96, May 2011. 
[12] Clayton P Jones. Slocum Glider Persistent Oceanography. In 2012 IEEE/OES Autonomous Underwater Vehicles (AUV), pages 1-6. IEEE, 2012.

[13] S G K Man, A B Phillips, S W Boyd, J I R Blake, and G Griffiths. Bio-inspired aquatic flight propulsion system for agile and manoeuverable underwater vehicles. OCEANS 2012 - YEOSU, pages 1-10, 2012.

[14] David Barrett, Mark A Grosenbaugh, and M Triantafyllou. The optimal control of a flexible hull robotic undersea vehicle propelled by an oscillating foil. In Autonomous Underwater Vehicle Technology, AUV'96, pages 1-9. IEEE, IEEE, 1996.

[15] Paul Riggs, Adrian Bowyer, and Julian Vincent. Advantages of a Biomimetic Stiffness Profile in Pitching Flexible Fin Propulsion. Journal of Bionic Engineering, 7(2):113-119, June 2010.

[16] G. S. Triantafyllou and Michael S Triantafyllou. An Efficient Swimming Machine. Scientific American, 272(3):64-70, 1995.

[17] Frank E Fish. Comparative kinematics and hydrodynamics of odontocete cetaceans: Morphological and ecological correlates with swimming performance. The Journal of Experimental Biology, 201(20):2867-2877, 1998.

[18] J E Colgate and K M Lynch. Mechanics and control of swimming: a review. IEEE Journal of Oceanic Engineering, 29(3):660-673, July 2004.

[19] Frank E Fish, Laurens E Howle, and Mark M Murray. Hydrodynamic flow control in marine mammals. Integrative and Comparative Biology, 48(6):788800, December 2008.

[20] Keith William Moored III, Frank E Fish, Trevor Kemp, and Hilary Bart-Smith. Batoid Fishes: Inspiration for the Next Generation of Underwater Robots. Marine Technology Society Journal, 2011.

[21] Yonas Tadesse, Alex Villanueva, Carter Haines, David Novitski, Ray Baughman, and Shashank Priya. Hydrogen-fuel-powered bell segments of biomimetic jellyfish. Smart Materials and Structures, 21(4):045013, March 2012.

[22] David Barrett, DKP Yue, Mark A Grosenbaugh, MJ Wolfgang, and Michael S Triantafyllou. Drag reduction in fish-like locomotion. Journal of Fluid Mechanics, 392:183-212, 1999.

[23] Jamie M Anderson and Narender K Chhabra. Maneuvering and stability performance of a robotic tuna. Integrative and Comparative Biology, 42(1):118-126, 2002 .

[24] Alberto Tonello. Control and guidance systems for the navigation of a biomimetic autonomous underwater vehicle. PhD thesis, 2011. 
[25] John Muir Kumph. Maneuvering of a robotic pike. Master's thesis, Massachusetts Institute of Technology, May 2005.

[26] Brenden P Epps, Pablo Valdivia y Alvarado, Kamal Youcef-Toumi, and Alexandra H Techet. Swimming performance of a biomimetic compliant fish-like robot. Experiments in Fluids, 47(6):927-939, 2009.

[27] James L Tangorra, Chris Phelan, Chris Esposito, and George V Lauder. Use of Biorobotic Models of Highly Deformable Fins for Studying the Mechanics and Control of Fin Forces in Fishes. Integrative and Comparative Biology, 51(1):176-189, 2011.

[28] J Gottlieb. The development of a multi-functional bio-robotic pectoral fin. Master's thesis, dspace.library.drexel.edu, 2009.

[29] Alexandra H Techet. Propulsive performance of biologically inspired flapping foils at high Reynolds numbers. The Journal of Experimental Biology, 211(2):274-279, January 2008.

[30] K H Low. Current and future trends of biologically inspired underwater vehicles. 2011 Defense Science Research Conference And Expo (DSR), pages 1-8, 2011.

[31] Naomi Kato. Median and Paired Fin Controllers for Biomimetic Marine Vehicles. Applied Mechanics Reviews, 58(4):238, 2005.

[32] S Vogel. Life in Moving Fluids: The Physical Biology of Flow. Princeton paperbacks. Princeton University Press, 1994.

[33] P Webb. Simple physical principles and vertebrate aquatic locomotion. Integrative and Comparative Biology, 1988.

[34] John J. Videler, Eize J. Stamhuis, Ulrike K Müller, and Luca A. van Duren. The Scaling and Structure of Aquatic Animal Wakes. Integrative and Comparative Biology, 42(5):988-996, November 2002.

[35] Michael H Dickinson. Unsteady mechanisms of force generation in aquatic and aerial locomotion. American zoologist, 36(6):537-554, 1996.

[36] Michael S Triantafyllou, A. H. Techet, Q Zhu, D N Beal, F. S. Hover, and D. K. P. Yue. Vorticity Control in Fish-Like Propulsion and Maneuvering. Integrative and Comparative Biology, 42(5):1026-1031, November 2002.

[37] Q Zhu, M Wolfgang, and D Yue. Three-dimensional flow structures and vorticity control in fish-like swimming. Journal of Fluid Mechanics, 2002.

[38] John O Dabiri. Optimal Vortex Formation as a Unifying Principle in Biological Propulsion. Annual Review of Fluid Mechanics, 41(1):17-33, 2009. 
[39] Keith William Moored III and Hilary Bart-Smith. Investigation of clustered actuation in tensegrity structures. International Journal of Solids \& Structures, 2009 .

[40] Keith William Moored III, Peter A Dewey, M Leftwich, Hilary Bart-Smith, and Alexander J Smits. Bioinspired Propulsion Mechanisms Based on Manta Ray Locomotion. Marine Technology Society Journal, 2011.

[41] R Clark, J Buchholz, and Alexander J Smits. Thrust production and wake structure of a batoid-inspired oscillating fin. Journal of Fluid Mechanics, 2006.

[42] R Love and A Arroyo. Solar Ray: An Autonomous Solar-Powered Bio-mimetic Flapping-Wing Underwater Vehicle. Florida Conference on Recent Advances in Robotics, FCRAR 2010, 2010.

[43] Theo PL Brower. Design of a manta ray inspired underwater propulsive mechanism for long range, low power operation. Master's thesis, July 2006.

[44] Jun Gao, Shusheng Bi, Yicun Xu, and Cong Liu. Development and design of a robotic manta ray featuring flexible pectoral fins. In Robotics and Biomimetics, 2007. ROBIO 2007. IEEE International Conference on, pages 519-523, 2007.

[45] Ronald A Forch. Exploring New Paradigms in Locomotion: The Development and Analysis of a Simplified Manta Ray Wing Model. Master's thesis, University of Virginia, 2009.

[46] S Yang and J Qiu. Kinematics Modeling and Experiments of Pectoral Oscillation Propulsion Robotic Fish. Journal of Bionic Engineering, 2009.

[47] Chunlin Zhou and Kin-Huat Low. Better Endurance and Load Capacity: An Improved Design of Manta Ray Robot (RoMan-II). Journal of Bionic Engineering, 7(S):S137-S144, October 2010.

[48] Robert Scott Russo. Biomechanical modeling of ray pectoral fins to inform the design of AUV propulsion systems. PhD thesis, December 2012.

[49] Gwynn Griffiths, Clayton Jones, James Ferguson, and Neil Bose. Undersea gliders. Journal of Ocean Technology, pages 1-11, September 2007.

[50] Andrea D Marshall, C L Dudgeon, and M B Bennett. Size and structure of a photographically identified population of manta rays Manta alfredi in southern Mozambique. Marine Biology, 158(5):1111-1124, 2011.

[51] Heidi Dewar, Peter Mous, Michael Domeier, Andreas Muljadi, Jos Pet, and Jeff Whitty. Movements and site fidelity of the giant manta ray, Manta birostris, in the Komodo Marine Park, Indonesia. Marine Biology, 155(2):121-133, June 2008 . 
[52] Simon J Pierce, Michael B Bennett, and Andrea D Marshall. Morphological measurements of manta rays (Manta birostris) with a description of a foetus from the east coast of Southern Africa. Zootaxa, (1717):24-30, 2008.

[53] Kazunari Yano, Fumihiko Sato, and Tomoko Takahashi. Observations of mating behavior of the manta ray, Manta birostris, at the Ogasawara Islands, Japan. Ichthyological Research, 46(3):289-296, 1999.

[54] L Rosenberger. Pectoral fin locomotion in batoid fishes: undulation versus oscillation. The Journal of Experimental Biology, 2001.

[55] Justin T Schaefer and Adam P Summers. Batoid wing skeletal structure: Novel morphologies, mechanical implications, and phylogenetic patterns. Journal of Morphology, 264(3):298-313, June 2005.

[56] Paul W Webb. The Biology of Fish Swimming. The Mechanics and Physiology of Animal Swimming. Cambridge University Press, 1994.

[57] L Rosenberger and Mark W Westneat. Functional morphology of undulatory pectoral fin locomotion in the stingray Taeniura lymma (Chondrichthyes: Dasyatidae). The Journal of Experimental Biology, 1999.

[58] C Heine. Mechanics of Flapping Fin Locomotion in the Cownose Ray, Rhinoptera bonasus (Elasmobranchii: Myliobatidae). PhD thesis, Dissertation, 1992.

[59] Jana M Parson, Frank E Fish, and Anthony J Nicastro. Turning performance of batoids: Limitations of a rigid body. Journal of Experimental Marine Biology and Ecology, 402(1-2):12-18, June 2011.

[60] M Sfakiotakis, DM Lane, and JBC Davies. Review of fish swimming modes for aquatic locomotion. IEEE Journal of Oceanic Engineering, 24(2):237-252, 1999.

[61] George V Lauder. Swimming hydrodynamics: ten questions and the technical approaches needed to resolve them. Experiments in Fluids, 51(1):23-35, July 2011.

[62] Kirill V Rozhdestvensky and Vladimir A Ryzhov. Aerohydrodynamics of flapping-wing propulsors. Progress in Aerospace Sciences, 39(8):585-633, November 2003.

[63] G. S. Triantafyllou, Michael S Triantafyllou, and Mark A Grosenbaugh. Optimal Thrust Development in Oscillating Foils with Application to Fish Propulsion. Journal of Fluids and Structures, 7(2):205-224, February 1993.

[64] G Taylor and R Nudds. Flying and swimming animals cruise at a Strouhal number tuned for high power efficiency. Nature, 2003. 
[65] Jamie M Anderson, K Streitlien, David Barrett, and Michael S Triantafyllou. Oscillating foils of high propulsive efficiency. Journal of Fluid Mechanics, 360(0):41-72, April 1998.

[66] M Koochesfahani. Vortical patterns in the wake of an oscillating airfoil. AIAA journal, 27(9):1200-1205, 1989.

[67] Teis Schnipper, Anders Andersen, and Tomas Bohr. Vortex wakes of a flapping foil. Journal of Fluid Mechanics, 633:411, August 2009.

[68] Frank E Fish, MK Nusbaum, JT Beneski, and DR Ketten. Passive cambering and flexible propulsors: cetacean flukes. Bioinspiration \& Biomimetics, 1:S42S48, 2006.

[69] Michael S Triantafyllou, G. S. Triantafyllou, and D. K. P. Yue. Hydrodynamics of Fishlike Swimming. Annual Review of Fluid Mechanics, 32(1):33-53, 2000.

[70] P Prempraneerach, F. S. Hover, and Michael S Triantafyllou. The effect of chordwise flexibility on the thrust and efficiency of a flapping foil. In Proc. th Int. Symp. on Unmanned Untethered Submersible Technology, pages 1-11, July 2003.

[71] S Heathcote, Z Wang, and I Gursul. Effect of spanwise flexibility on flapping wing propulsion. Journal of Fluids and Structures, 2008.

[72] Michael S Triantafyllou, A. H. Techet, and F. S. Hover. Review of Experimental Work in Biomimetic Foils. IEEE Journal of Oceanic Engineering, 29(3):585594, July 2004.

[73] James L Tangorra, George V Lauder, and P Madden. A biorobotic flapping fin for propulsion and maneuvering. IEEE International Conference on Robotics and Automation, 2008.

[74] James L Tangorra, George V Lauder, Ian W Hunter, Rajat Mittal, Peter G A Madden, and Meliha Bozkurttas. The effect of fin ray flexural rigidity on the propulsive forces generated by a biorobotic fish pectoral fin. The Journal of Experimental Biology, 213(23):4043-4054, December 2010.

[75] J Palmisano, R Ramamurti, K Lu, and J Cohen. Design of a Biomimetic Controlled-Curvature Robotic Pectoral Fin. 2007 IEEE International Conference on Robotics and Automation, 2007.

[76] A Willy and K Low. Development and initial experiment of modular undulating fin for untethered biorobotic AUVs. IEEE International Conference on Robotics and Biomimetics (ROBIO), 2005.

[77] K H Low. Locomotion Consideration and Implementation of Robotic Fish with Modular Undulating Fins: Analysis and Experimental Study. In Intelligent Robots and Systems, 2006 IEEE/RSJ International Conference on, pages $2424-$ 2429, 2006. 
[78] K H Low and A Willy. Biomimetic Motion Planning of an Undulating Robotic Fish Fin. Journal of Vibration and Control, 12(12):1337-1359, December 2006.

[79] Michael Epstein, J Edward Colgate, and Malcolm A MacIver. Generating Thrust with a Biologically-Inspired Robotic Ribbon Fin. In Intelligent Robots and Systems, IEEERSJ International Conference on, pages 2412-2417, February 2006.

[80] Oscar M Curet, Neelesh A Patankar, George V Lauder, and Malcolm A MacIver. Mechanical properties of a bio-inspired robotic knifefish with an undulatory propulsor. Bioinspiration \& Biomimetics, 6(2):026004, April 2011.

[81] Peter A Dewey, Antoine Carriou, and Alexander J Smits. On the relationship between efficiency and wake structure of a batoid-inspired oscillating fin. Journal of Fluid Mechanics, 691:245-266, December 2011.

[82] Wanchao Chi and Kin-Huat Low. Review and Fin Structure Design for Robotic Manta Ray (RoMan IV). Journal of Robotics and Mechatronics, 24(4):620-628, 2012 .

[83] Sean Andrew Mellott. Design of an Actuation Mechanism for Compliant-body Biomimetic Robots. PhD thesis, Massachusetts Institute of Technology, 2009.

[84] W. Stoll. Aqua_ray: Water-hydraulic manta ray with flapping-wing drive, 2007. http://www.festo.com/cms/en_corp/9786.htm.

[85] Gang Wang, Longxin Lin, Haijun Xu, Haibin Xie, and Lincheng Shen. Bionic Bladder Based Depth Control for Bionic Underwater Robots. In Control and Automation ICCA, 8th IEEE International Conference on, pages 1-5, July 2010.

[86] K Low. Modelling and parametric study of modular undulating fin rays for fish robots. Mechanism and Machine Theory, 2009.

[87] K Suzumori, S Endo, T Kanda, N Kato, and H Suzuki. A Bending Pneumatic Rubber Actuator Realizing Soft-bodied Manta Swimming Robot. In Robotics and Automation, 2007 IEEE International Conference on, pages 4975-4980, 2007.

[88] Z Wang, Y Wang, and J Li. A micro biomimetic manta ray robot fish actuated by SMA. IEEE International Conference on Robotics and Biomimetics, 2010.

[89] Won-Shik Chu, Kyung-Tae Lee, Sung-Hyuk Song, Min-Woo Han, Jang-Yeob Lee, Hyung-Soo Kim, Min-Soo Kim, Yong-Jai Park, Kyu-Jin Cho, and SungHoon Ahn. Review of biomimetic underwater robots using smart actuators. International Journal of Precision Engineering and Manufacturing, 13(7):12811292, July 2012.

[90] K H Low, Chunlin Zhou, Gerald Seet, Shusheng Bi, and Yueri Cai. Improvement and testing of a robotic manta ray (RoMan-III). IEEE International Conference on Robotics and Biomimetics, pages 1730-1735, 2011. 
[91] Chunlin Zhou and K H Low. Design and Locomotion Control of a Biomimetic Underwater Vehicle With Fin Propulsion. IEEE/ASME Transactions on Mechatronics, 17(1):25-35, 2012.

[92] Yueri Cai, Shusheng Bi, Lige Zhang, and Jun Gao. Design of a robotic fish propelled by oscillating flexible pectoral foils. In Intelligent Robots and Systems, 2009. IROS 2009. IEEE/RSJ International Conference on, pages 2138-2142, 2009.

[93] Yueri Cai, Shusheng Bi, and Lige Zhang. Design and Implication of a Bionic Pectoral Fin Imitating Cow-Nosed Ray. In Intelligent Robots and Systems IROS, IEEERSJ International Conference on, pages 1-5. IEEE, July 2010.

[94] Shusheng Bi, Yueri Cai, and Licheng Zheng. Design and Experiments of a Robotic Fish Imitating Cow-Nosed Ray. Journal of Bionic Engineering, 2010.

[95] R Motro. Tensegrity: Structural Systems for the Future. Elsevier Science, 2003.

[96] Donald E Ingber. The architecture of life. Scientific American, 278(1):48-57, 1998.

[97] E Hartford and Cornel Sultan. Tensegrity Structures Research Evolution. Decision and Control, 2006.

[98] Robert E Skelton, R Adhikari, J.-P Pinaud, Waileung Chan, and J.W Helton. An introduction to the mechanics of tensegrity structures, volume 5 of Decision and Control, 2001. Proceedings of the 40th IEEE Conference on. IEEE, IEEE, 2001.

[99] C R Calladine. Buckminster Fuller's "tensegrity" structures and Clerk Maxwell's rules for the construction of stiff frames. International Journal of Solids \& Structures, 14(2):161-172, 1978.

[100] E Fest, K Shea, Bernd Domer, and Ian FC Smith. Adjustable Tensegrity Structures. Journal of Structural Engineering, 2003.

[101] T Sterk. Using Actuated Tensegrity Structures to Produce a Responsive Architecture. ACADIA22 Conference Proceedings, 2003.

[102] Jean-Paul Pinaud, Milenko Masic, and Robert E Skelton. Path planning for the deployment of tensegrity structures. Smart Structures and Materials, 5049:436447, August 2003.

[103] E Fest, K Shea, and Ian FC Smith. Active Tensegrity Structure. Journal of Structural Engineering, 2004.

[104] J.B Aldrich, Robert E Skelton, and K Kreutz-Delgado. Control synthesis for a class of light and agile robotic tensegrity structures. 2003 American Control Conference, 6:5245-5251 vol.6, 2003. 
[105] Jean-Paul Pinaud, Soren Solari, and Robert E Skelton. Deployment of a class 2 tensegrity boom. Smart Structures and Materials, 5390:155-162, 2004.

[106] Bernd Domer and Ian FC Smith. An active structure that learns. Journal of Computing in Civil Engineering, 19(1):16-24, 2005.

[107] Chandana Paul, Francisco J Valero-Cuevas, and Hod Lipson. Design and control of tensegrity robots for locomotion. Robotics, IEEE Transactions on, 22(5):944957, 2006.

[108] Mizuho Shibata, Fumio Saijyo, and Shinichi Hirai. Crawling by body deformation of tensegrity structure robots. In Robotics and Automation, . ICRA. IEEE International Conference on, pages 4375-4380. IEEE, 2009.

[109] Thomas K Bliss, Jeffrey Werly, Tetsuya Iwasaki, and Hilary Bart-Smith. Experimental validation of robust resonance entrainment for CPG-controlled tensegrity structures. Control Systems Technology, IEEE Transactions on, 21(3):666-678, 2013.

[110] Thomas K Bliss, Tetsuya Iwasaki, and Hilary Bart-Smith. Central Pattern Generator Control of a Tensegrity Swimmer. IEEE/ASME Transactions on Mechatronics, 18(2):586-597, 2013.

[111] Keith William Moored III, S Taylor, Thomas K Bliss, and Hilary Bart-Smith. Optimization of a tensegrity wing for biomimetic applications. Decision and Control, 2006.

[112] Keith William Moored III and Hilary Bart-Smith. The Analysis of Tensegrity Structures for the Design of a Morphing Wing. Journal of Applied Mechanics, 2007.

[113] Nathan E Houle. Design, Manufacturing and Experimental Validation of Class 2 Tensegrity Structures for Application in a Bio-inspired Flapping Wing. Master's thesis, 2009.

[114] B de Jager and Robert E Skelton. Stiffness of planar tensegrity truss topologies. International Journal of Solids \& Structures, 43(5):1308-1330, 2006.

[115] E. Oberg, F.D. Jones, H.L. Horton, C.J. McCauley, and H.H. Ryffel. Machinery's Handbook. Machinery's Handbook. Industrial Press, 2004.

[116] ASTM Standard A931. Test Method for Tension Testing of Wire Ropes and Strand. ASTM International, 2008. DOI: 10.1520/A0931-08R13, .

[117] James Stewart. Calculus. Brooks/Cole Pub Co, 4th edition, 1999.

[118] George V Lauder and Peter G A Madden. Fish locomotion: kinematics and hydrodynamics of flexible foil-like fins. Experiments in Fluids, 43(5):641-653, 2007. 
[119] Frank E Fish and George V Lauder. Passive and active flow control by swimming fishes and mammals. Annual Review of Fluid Mechanics, 38:193-224, 2006.

[120] C Fiazza, T Salumae, M Listak, G Kulikovskis, R Templeton, O Akanyeti, William Megill, P Fiorini, and M Kruusmaa. Biomimetic mechanical design for soft-bodied underwater vehicles. In OCEANS IEEE-Sydney, pages 1-7, 2010.

[121] George V Lauder, Brooke Flammang, and S Alben. Passive Robotic Models of Propulsion by the Bodies and Caudal Fins of Fish. Integrative and Comparative Biology, 52(5):576-587, October 2012.

[122] Brian Gratwicke. Spotted eagle ray. Feb. 13, 2013 via Flickr, Creative Commons Attribution.

[123] Daniel B Quinn, Keith William Moored III, Peter A Dewey, and Alexander J Smits. Unsteady propulsion near a solid boundary. Journal of Fluid Mechanics, 742:152-170, February 2014.

[124] Daniel B Quinn, George V Lauder, and Alexander J Smits. Flexible propulsors in ground effect. Bioinspiration \& Biomimetics, 9(3):036008, March 2014.

[125] M J Lighthill. Note on the swimming of slender fish. Journal of Fluid Mechanics, 9(02):305-317, May 1960.

[126] Vance A Tucker. Energetic cost of locomotion in animals. Comparative Biochemistry and Physiology, 34(4):841-846, June 1970.

[127] H H Ku. Notes on the use of propagation of error formulas. Journal of Research of the National Bureau of Standards, 70(4), 1966.

[128] George V Lauder, Erik J Anderson, James L Tangorra, and P G A Madden. Fish biorobotics: kinematics and hydrodynamics of self-propulsion. The Journal of Experimental Biology, 210(16):2767-2780, August 2007.

[129] ASTM Standard D638. Test Method for Tensile Properties of Plastics. ASTM International, 2008. DOI: 10.1520/D0638-10, .

[130] J Long, M Hale, and M McHenry. Functions of fish skin: flexural stiffness and steady swimming of longnose gar Lepisosteus osseus. The Journal of Experimental Biology, 1996.

[131] George V Lauder and Erin Blevins. Rajiform locomotion: three-dimensional kinematics of the pectoral fin surface during swimming in the freshwater stingray Potamotrygon orbignyi. The Journal of Experimental Biology, 215(18):32313241, August 2012.

[132] Keith William Moored III, Peter A Dewey, Hossein Haj-Hariri, and Alexander J Smits. Hydrodynamic wake resonance as an underlying principle of efficient unsteady propulsion. Journal of Fluid Mechanics, 708:329-348, August 2012. 
[133] Peter Dewey, Birgitt Boschitsch, and Alexander Smits. Effects of Flexibility on Bio-inspired Aquatic Propulsion. Bulletin of the American Physical Society, 57, 2012 . 